\title{
THE EFFECT OF PROTOZOAN GRAZERS ON THE CYCLING OF POLYCHLORINATED BIPHENYLS (PCBs) IN MARINE SYSTEMS
}

\author{
By \\ Elizabeth Belle Kujawinski \\ S.B., Massachusetts Institute of Technology, 1994 \\ Submitted in partial fulfillment of the requirements for the degree of \\ DOCTOR OF PHILOSOPHY \\ at the
MASSACHUSETTS INSTITUTE OF TECHNOLOGY
and the
WOODS HOLE OCEANOGRAPHIC INSTITUTION

February 2000

(C) 2000 Elizabeth B. Kujawinski

All rights reserved.

The author hereby grants to MIT and WHOI permission to reproduce paper and electronic copies of this thesis in whole or in part and to distribute them publicly.

Signature of Author

Joint Program in Oceanography

Massachusetts Institute of Technology

and Woods Hole Oceanographic Institution

February 2000

Certified by

John W. Farringter .

Cothesis
James W. Moffett Co-thesis supervisor

Accepted by

Margaret Tivey

Chair, Joint Committee for Chemical Oceanography

Massachusetts Institute of Technology/

Woods Hole Oceanographic Institution 


\section{Thesis Abstract}

Processes affecting organic carbon distribution and composition can control the speciation of organic contaminants such as polychlorinated biphenyls (PCBs) and ultimately determine their residence time in a particular environment. In marine systems, the microbial loop influences organic carbon dynamics by recycling a significant fraction of dissolved and particulate organic matter. The goal of this thesis was to understand how these recycling processes affect chlorobiphenyl (CB) cycling in marine systems by monitoring $\mathrm{CB}$ dynamics among organic carbon pools represented by dissolved organic matter, bacterial prey and phagotrophic protozoan grazers.

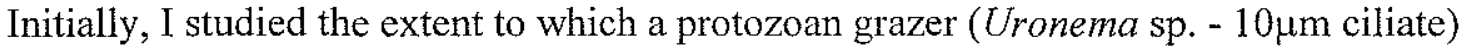
equilibrated with aqueous PCBs within 2-3 hours. Initial calculations predicted rapid equilibration via passive diffusion. Experimentally, no difference in equilibration time was noted between grazing and non-grazing protozoa, indicating that diffusion was the primary uptake pathway for these organisms. The results were extended to determine the transition size of an organism where the rates of diffusive and ingested uptake are equivalent (100-500 $\mu \mathrm{m})$. Disassociation rate constants were estimated for complexes of $\mathrm{CB}$ congeners and dissolved organic carbon (DOC). $\mathrm{CB}-\mathrm{DOC}$ complexes enhanced the diffusive uptake rate constant for Tenax resin and, by inference, protozoan grazers.

In the second phase of this work, concentrations of surfactants, organic carbon and cells were monitored over time in protozoan cultures. The effects of bacterial growth substrate and protozoan species were examined. Surfactants increased during protozoan exponential growth while total DOC concentrations decreased. Production of surfaceactive material in ciliate cultures was significantly higher than in flagellate cultures, and all protozoan cultures were higher than the bacterial control.

Common headspace vessels were then used to compare and contrast the affinity of protozoan and bacterial culture filtrates $(<0.2 \mu \mathrm{m})$ for PCBs relative to a seawater control. Affinities were normalized to bulk DOC and surfactant concentrations to determine underlying relationships among these parameters. Values of equilibrium partition coefficients $\left(\mathrm{K}_{\mathrm{oc}}\right)$ ranged from $10^{4.6}$ in Vineyard Sound seawater to $10^{5.4}$ and $10^{5.5} \mathrm{in}$ protist cultures, indicating that "grazer-enhanced" DOM was a better sorbent for PCBS than DOM in bacterial controls and Vineyard Sound seawater. 


\section{ACKNOWLEDGMENTS}

Many thanks are due to the numerous individuals who have helped make the Joint Program an educational and challenging experience.

First and foremost, I'd like to thank both my advisors, Jim Moffett and John Farrington, for their support and generosity. Thanks to Jim for taking on a project so outside his expansive realm of expertise and having the patience to help me find the way to the end. Thanks to John who became a mentor and wonderful role model through great discussions, moments of inspiration and supportive professional advice. Second, I'd like to thank my committee, Bruce Brownawell, Dave Caron and Phil Gschwend, whose advice and support is evident throughout this work. Thanks are especially due to Phil who introduced me to chemical oceanography at WHOI, cajoled me into applying to the Joint Program, and critically debated all my scientific and professional ideas throughout my tenure here. I would also like to thank Kathleen Ruttenberg for agreeing to be the chair of my defense. I could not have asked for a better advocate in these last few months.

This work would not have been possible without the generous support of many people here at WHOI and beyond. I feel I must thank the entire building of Redfield since I spent so much time there and have accosted nearly every member at one time or another with questions, concerns and requests. Many, many thanks are due to Dave Caron and his lab for all their help: Dave Caron, who taught me so much about protozoa and offered me access to his lab facilities and equipment, Mark Dennett, who answered so many lastminute questions with aplomb and kindness and Dawn Moran, who gave me many protozoan inocula with unending patience. The radioactive work presented here would not have been possible without the generosity of John Waterbury and Alan Fleer. All the DOC samples were run in the lab of Bob Chen at UMass-Boston with the help of Penny Vlahos. The Education Office (especially Stella, Julia, and Marsha) has provided invaluable assistance in navigating the worlds of MIT and WHOI.

Thanks so much to the group here in Fye. "Dr. Bob" Nelson answered innumerable analytical questions, tied countless knots, and offered pearls of wisdom at bleak hours (while calling me a variety of creative names, I have to add). Chris "Dr. Love" Reddy debated all sorts of wacky ideas, gave good analytical advice and made me laugh. Lorraine Eglinton was constantly helping me hone my mechanic's skills during times of instrument "down-time". Nelson Frew was incredibly generous with his time and equipment while I was working on the surfactant segment of this thesis. To everyone else (especially Sean, Dan, and Jean) thanks for fostering the work environment that allowed me to develop ideas and work independently.

Thanks to the Falmouth Inter-faith Choir and the Women's Committee for showing me that there was a life outside of the Joint Program. Thanks are especially due to Lauren 
Mullineaux who has exemplified my ideal female scientist and reminded me so many times that I had the potential to get there.

On the personal side, I have been truly blessed with friends and colleagues that I respect and cherish. Mak and Kathy B shared my lab and my office. Their scientific acumen, good nature and ability to laugh at themselves (and me) are most appreciated. Lihini, Ann, and Anna (and Maia) have in their own ways donated part of themselves and taught me a great deal about life and its priorities. Larry, Nicole, Derek, Sheri, Judith, Domenique, and the pets (Fred, Ginger, Jezebel, Sara Dawg, Bronski, Luna) shared my house and helped me have a haven I could call home. The many members of WHOI94 (especially Sheri (and Meghan), Sean (and Jess), Joe, Omak, Lou, and Sooze) have shared countless joyous experiences. Mike B and Maria Hood are also thanked for friendship and smiles.

In particular, I must thank my bevy of "guardian angels". To the members of the Thesis Support Group (Kirsten, Eli, Brenda, Sheri and Nicole), your kindness and support on Fridays over the last year have made the weeks shorter and the weekends brighter. I will never forget the laughs at "our" table and the supportive Kidd experience. To my non-JP friends, Kathy, Jackie, Vicki and Scott, thanks for reminding me who I was before grad school and giving me an escape when it got to be too much. To Tom, thanks for believing this was possible and providing wonderful distractions along the way. To Steve, I could not have asked for a better housemate or a truer friend. To Brenda, thanks for your unwavering support and seemingly endless strength - so much of this is due to you.

And finally, and most importantly, I'd like to thank my family. To Aunt Julia and Uncle Jim, thanks for the lovely get-away from the craziness of New England complete with great needlework advice and stimulating sports conversation. To Pete, my biggeryounger brother, thanks for the numerous reality checks and wonderful visits. To Dan, my younger-younger brother, thanks for sharing my sense of humor and supporting my assertion that Chicago is, indeed, the best. To my dearest parents, you are my staunchest supporters, closest friends, and greatest inspirations. Words cannot express the depth of my gratitude for your love.

This thesis is dedicated to my grandmother who taught me to roller-skate uphill.

The work described herein was supported by National Science Foundation Contract No. OCE-9253910 and Office of Naval Research AASERT Grant No. N00014-96-1-0718. 


\section{TABLE OF CONTENTS}

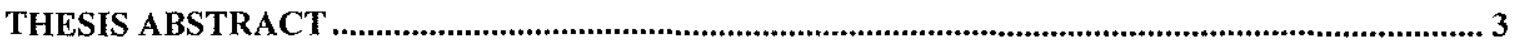

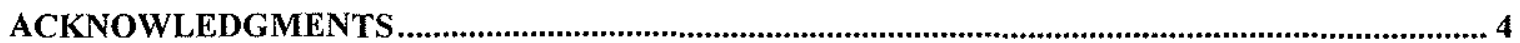

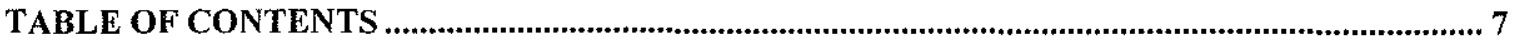

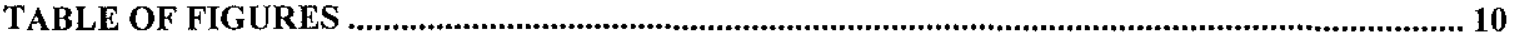

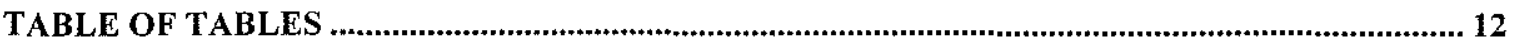

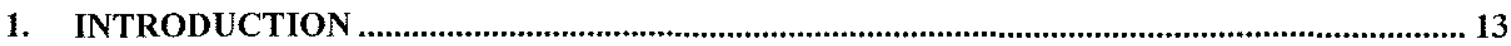

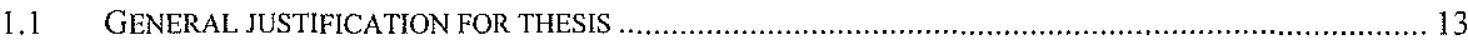

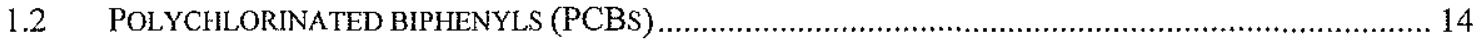

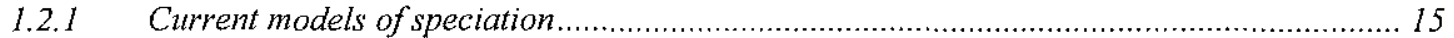

1.2.2 Effect of structure and chemical composition on $C B$ sorption to organic matter ................... 18

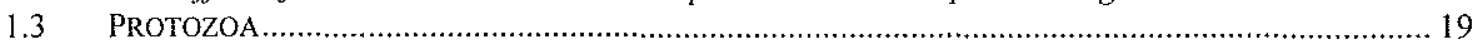

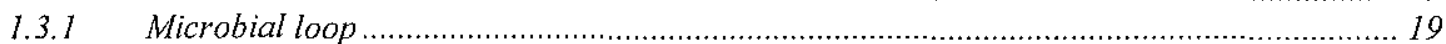

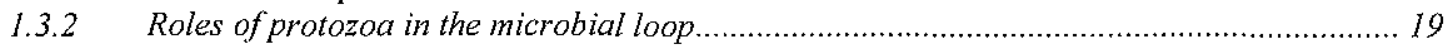

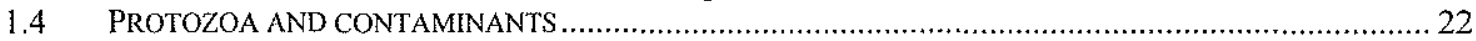

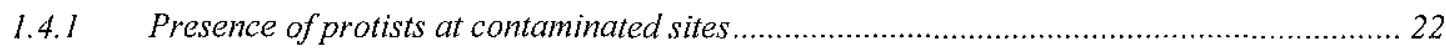

1.4.2 Field studies suggesting importance of microbial loop ……….............................................. 23

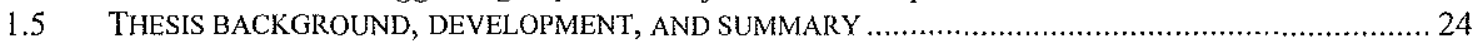

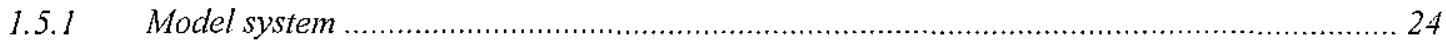

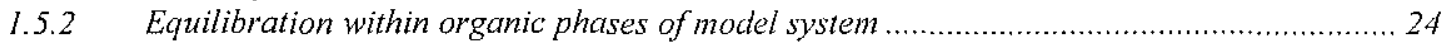

1.5.3 Production of material by protists and its affinity for PCBs........................................ 25

\section{THE IMPORTANCE OF PASSIVE DIFFUSION IN THE UPTAKE OF PCBS BY}

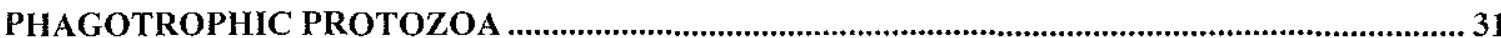

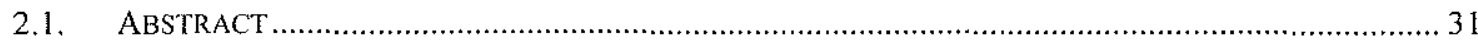

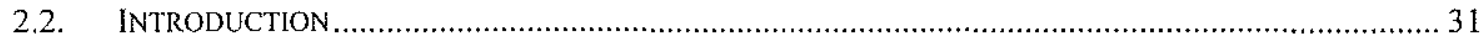

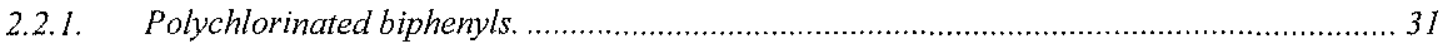

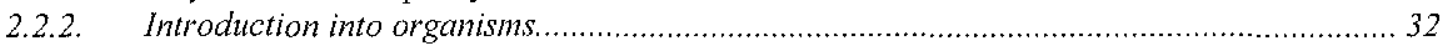

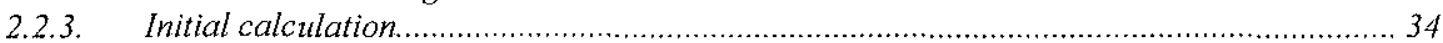

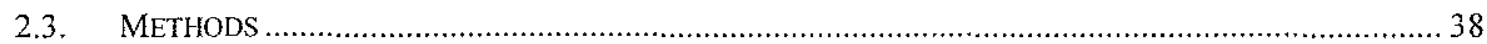

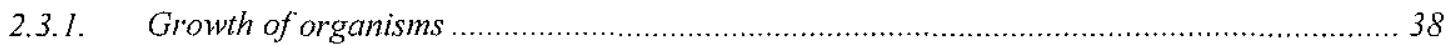

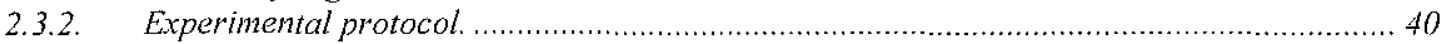

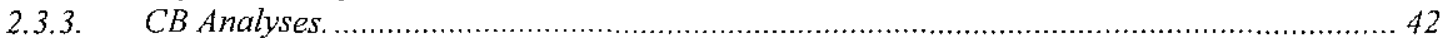

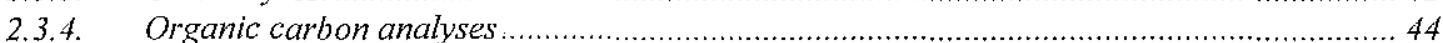

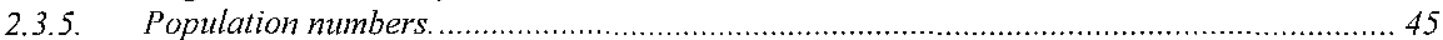

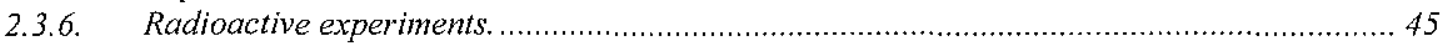

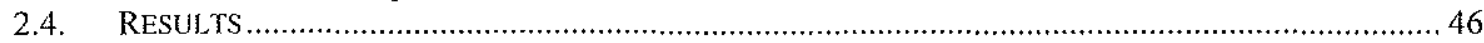

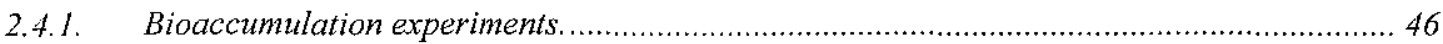

2.4.2. Comparison of $C B$ aqueous concentrations to $C B$ aqueous solubilities............................... 48

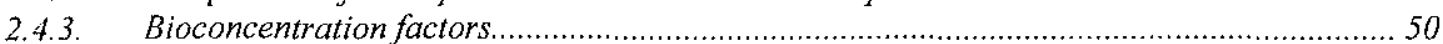

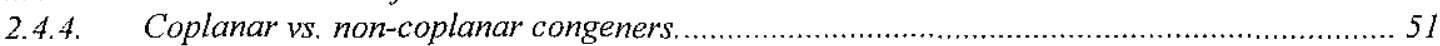

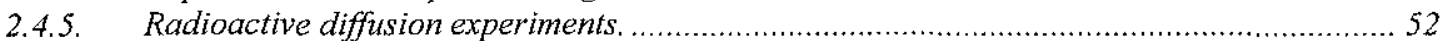

2.4.6. Calculation of bacterial loss rate constant .................................................................... 53

2.4.7. Comparison of protozoan uptake rate and bacterial depuration rate ................................54

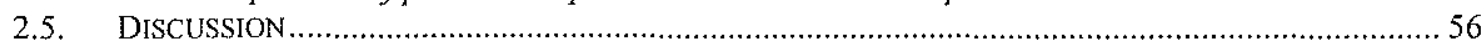

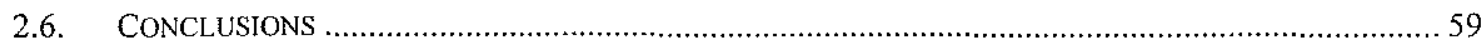




\section{EVIDENCE FOR DOC-ENHANCED MOLECULAR DIFFUSION IN A BACTERIAL}

\section{CULTURE}

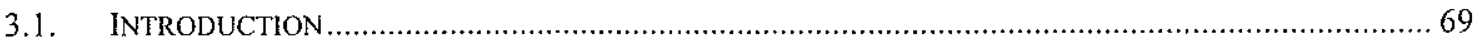

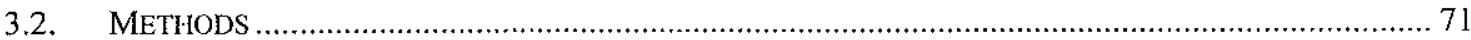

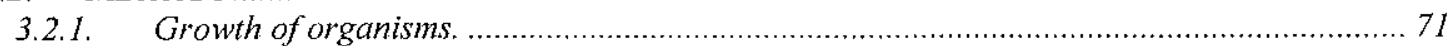

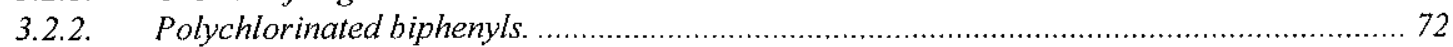

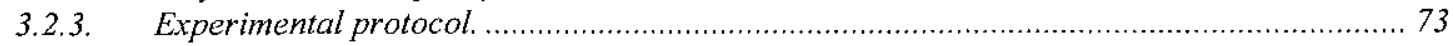

3.2.4. Ancillary measurements - bacterial concentrations and DOC................................... 74

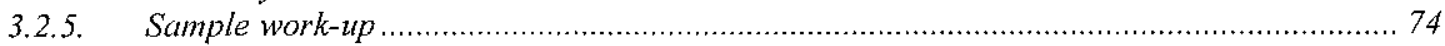

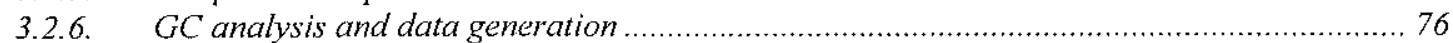

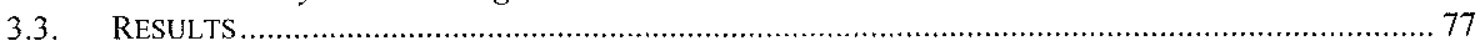

3.3.1. Calculation of expected and measured extraction rate constants...................................... 77

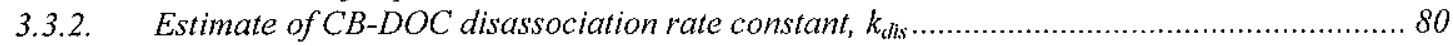

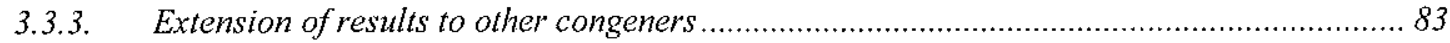

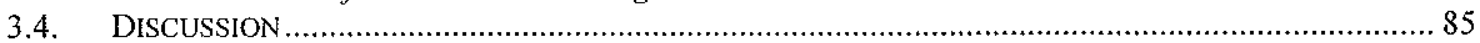

3.4.1. Implications for protozoan uptake of PCBs and microbial food web ................................. 85

3.4.2. Implications for the concept of "bioavailability" ........................................................ 86

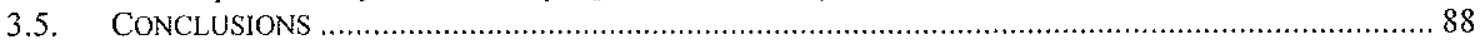

\section{DISSOLVED ORGANIC MATTER CYCLING IN PROTOZOAN GRAZING CULTURES:}

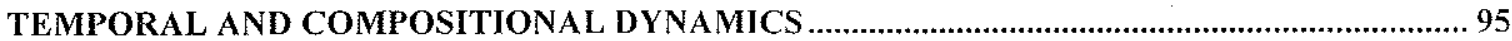

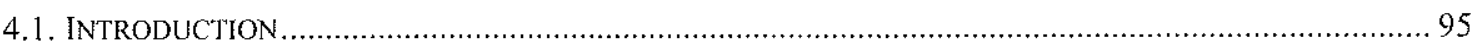

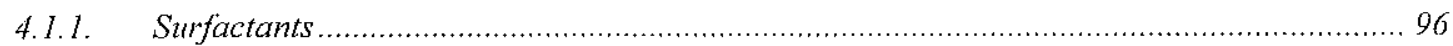

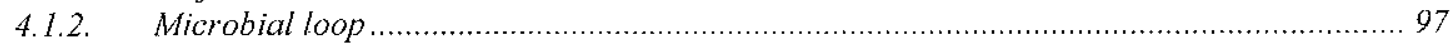

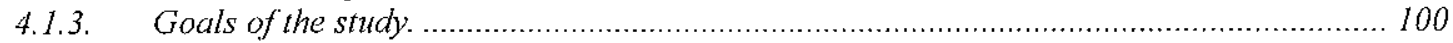

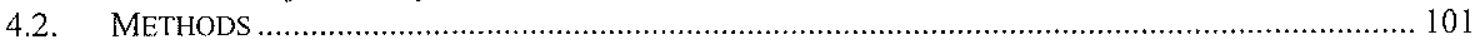

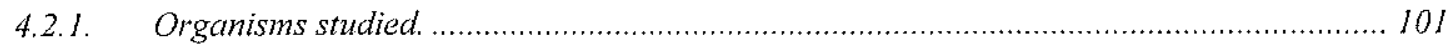

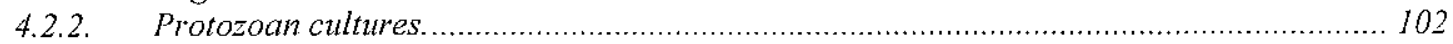

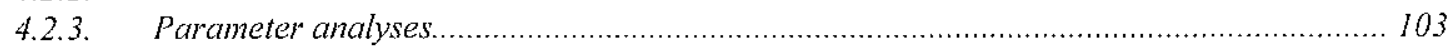

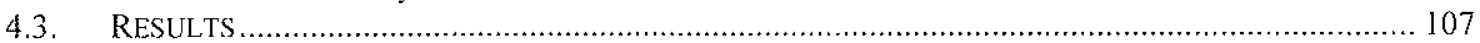

4.3.1. Comparison of methods used for collection of dissolved samples, ................................ 107

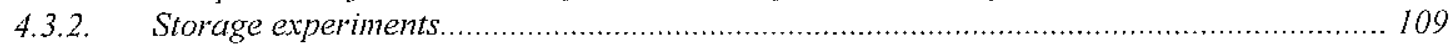

4.3.3. Initial studies with Uronema and H.halodurans..................................................... 110

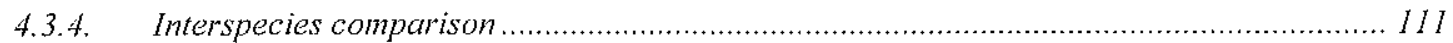

4.3.3. Ingestion and surfactant production rates .............................................................

4.3.4. Lipid data-bulk and compositional information .................................................... 115

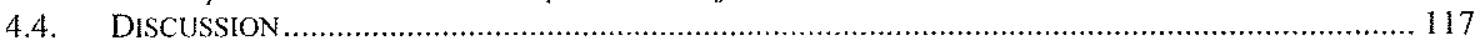

4.4.1. Relative dynamics of organic carbon, surfactants and lipids ..................................... 117

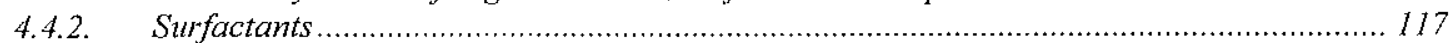

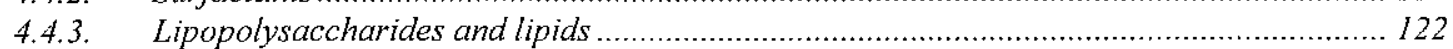

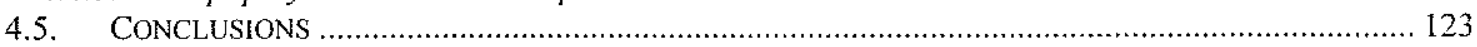

\section{EFFECT OF DOC COMPONENTS ON CB SPECIATION IN PROTOZOAN CULTURE}

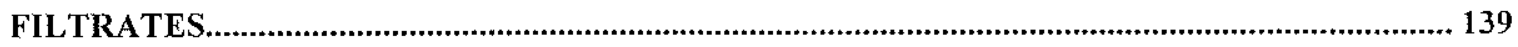

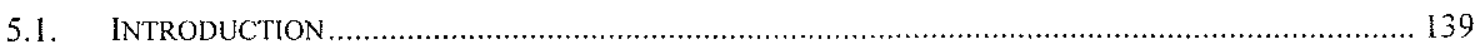

5.1.1. $\quad C B$ speciation in natural waters - "bioavailable" fraction ……................................ 139

5.I.2. Potential role of microbial loop (and protozoa) in CB speciation .................................. 141

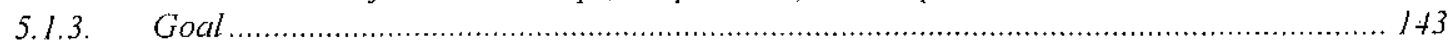

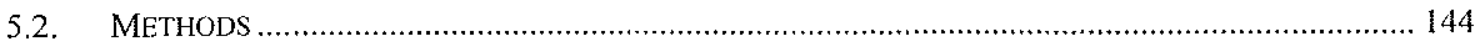

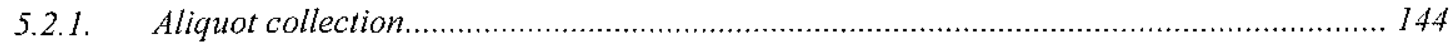

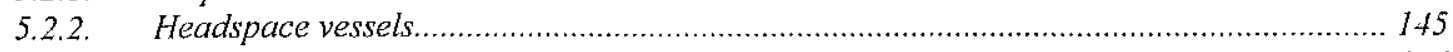

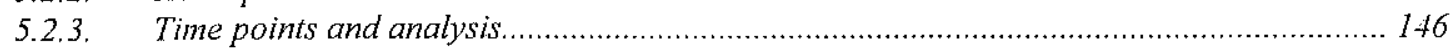




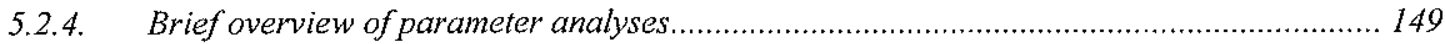

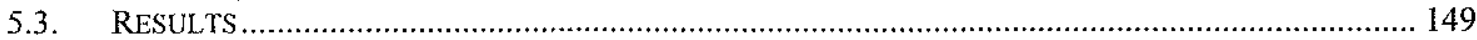

5.3.1. Partition coefficients $\left(K_{D O C}\right)$ in culture filtrates .................................................... 149

5.3.2. Comparison of binding potential with bulk DOC and surfactants................................ 152

5.3.2. Calculation of potential lipid contribution ............................................................... 152

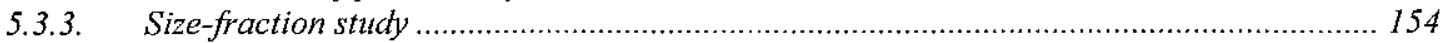

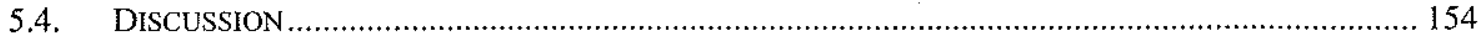

5.4.2. Comparison of grazing filtrates to bacterial and VSW controls.................................. 154

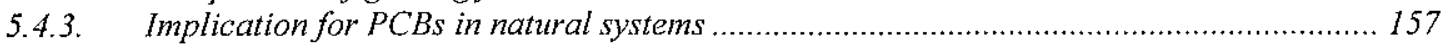

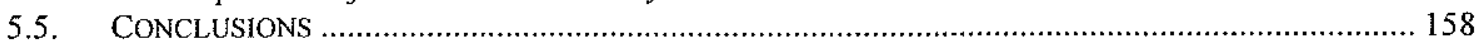

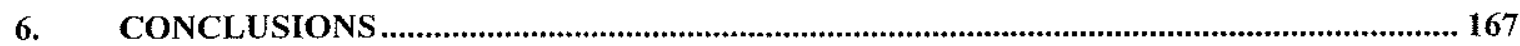

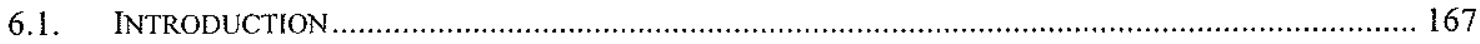

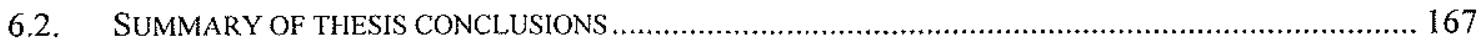

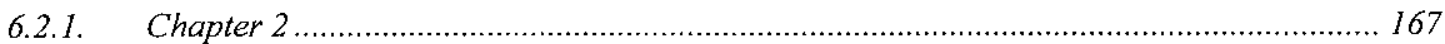

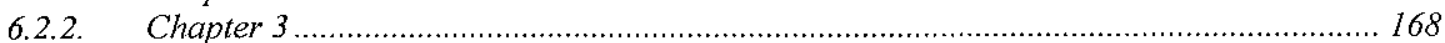

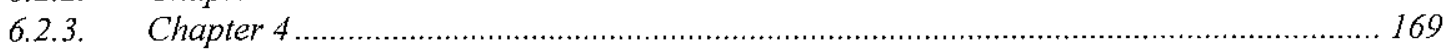

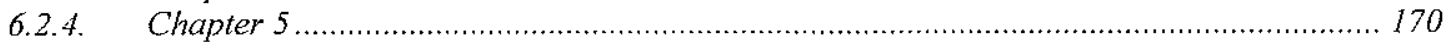

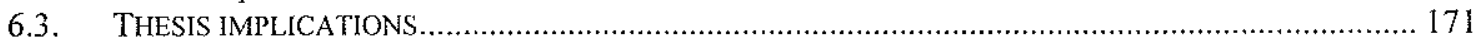

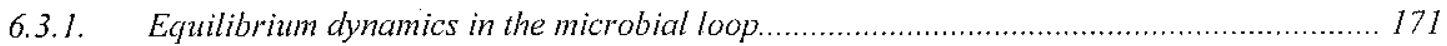

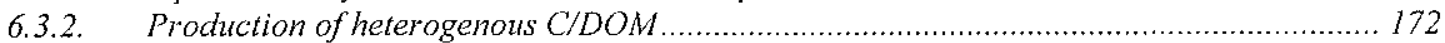

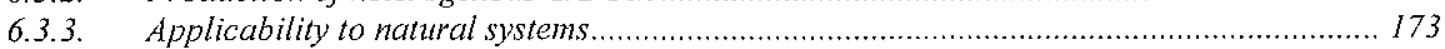

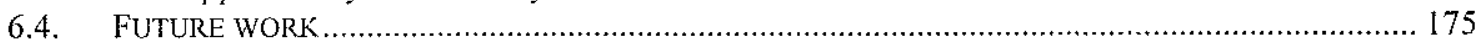

APPENDIX A - EFFECT OF BOUNDARY LAYER ON DIFFUSIVE UPTAKE OF PCBS BY

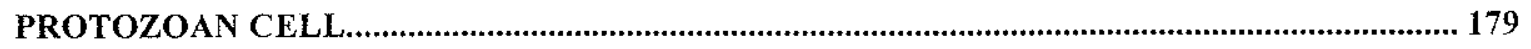

APPENDIX B - DATA TABLES FOR CHAPTER 2 BIOACCUMULATION EXPERIMENT ..... 183

APPENDIX C - CORRECTION TO EQUATION IN CORNELISSEN ET AL. (1997)................203

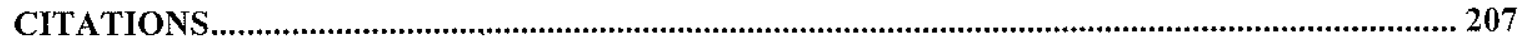




\section{TABLE OF FIGURES}

\section{Chapter 1}

Figure 1-1. Conceptual model of CB speciation in aquatic systems......................................................2 27

Figure 1-2. Carbon and nutrient cycles in model system containing protozoa and bacterial prey..............22

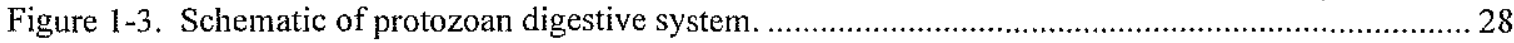

Figure 1-4. Protozoan and bacterial dynamics at a jet-fuel contaminated site...........................................29

Figure 1-5. Ratio of depositional fluxes to sedimentation rates for selected $\mathrm{CB}$ congeners and polyaromatic

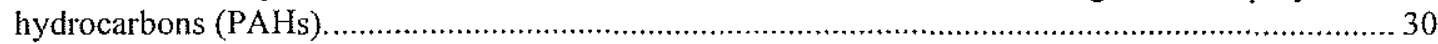

\section{Chapter 2}

Figure 2-1. Total recovery for IUPAC \#1 87 versus volatilization model..................................................6 60

Figure 2-2. Masses of selected congeners retained on 5.0 $\mu \mathrm{m} \mathrm{Ag}$ filter as a function of time. .................... 61

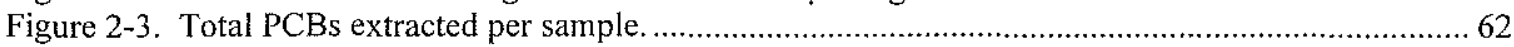

Figure 2-4. Fraction of three selected congeners $(18,128,195)$ retained on $5.0 \mu \mathrm{m}$ filter vs. time............ 63

Figure 2-5. Bioconcentration factors for each congener in the experimental flasks.................................. 64

Figure 2-6. Ratio of coplanar to non-coplanar PCBs in bioaccumulation experiment. .............................. 65

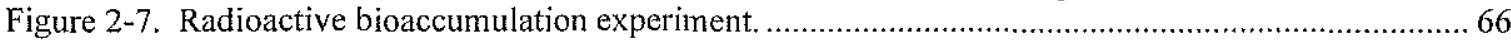

Figure 2-8. Non-linear regression fits for data from radioactive experiment. ........................................67

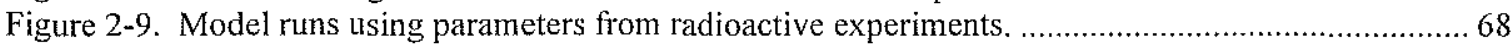

\section{Chapter 3}

Figure 3-1. Schematic detailing enhanced diffusion due to CB/DOC interactions....................................90

Figure 3-2. Expected and measured extraction rate constants versus $\log K_{\text {ow }}$ for both incubations........... 91

Figure 3-3. Expected and measured rate constants (predicted) for $3 \mathrm{CB}$ congeners as a function of DOC. 93

\section{Chapter 4}

Figure 4-1. Organic carbon cycle in surface ocean as result of food web cycling.................................. 124

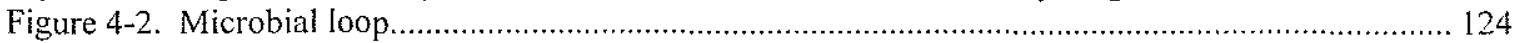

Figure 4-3. Example of results obtained by surfactant method. .................................................... 125

Figure 4-4. Collection method study for dissolved parameters - surfactants, LPS, and DOC ............... 127

Figure 4-5. Collection study for Uronema culture - syringe filtration vs. centrifugation. ....................... 127

Figure 4-6. Storage experiment for H.halodurans and Uronema surfactant samples............................. 128

Figure 4-7. Lipopolysaccharide storage experiment for H.halodurans culture. ................................... 129

Figure 4-8. Surfactant concentrations and Uronema \# versus time in two early experiments.................. 130

Figure 4-9. Surfactant concentrations in a Uronema culture with glucose-grown prey. ......................... 131

Figure 4-10. Surfactant concentrations in a Uronema and a Cafeteria culture with pyruvate-grown prey.

Figure 4-11. Data for interspecies comparison - Hhalodurans - population, DOC, LPS, and surfactants.

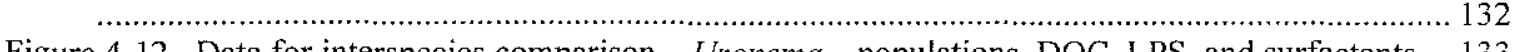

Figure 4-12. Data for interspecies comparison - Uronema - populations, DOC, LPS, and surfactants. .. 133

Figure 4-13. Data for interspecies comparison - Cafeteria - population, DOC, LPS, and surfactants. ... 134 Figure 4-14. Data for interspecies comparison - P. Imperforata - population, DOC, LPS, and surfactants.

Figure 4-15. Surfactant data for all cultures in interspecies comparison.............................................. 136

Figure 4-16. DOC data for all cultures in interspecies comparison.................................................... 136

Figure 4-17. LPS data for all cultures in interspecies comparison. .................................................. 137

Figure 4-18. Comparison of surfactant production rates and ingestion rates in 3 protozoan species......... 138

\section{Chapter 5}

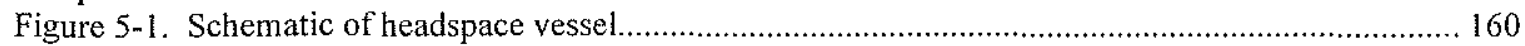

Figure 5-2. Representative figures of different headspace experiments. ................................................. 162

Figure 5-3. Binding data for all data versus bulk DOC concentrations with calculated $\log \mathrm{K}_{\mathrm{oc}} \ldots \ldots \ldots \ldots \ldots . . . . .163$ 


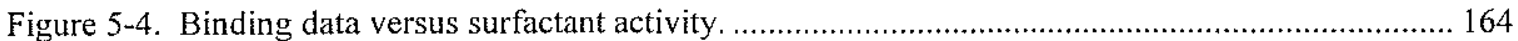

Figure 5-5. Binding data versus bulk DOC concentrations. .................................................... 164

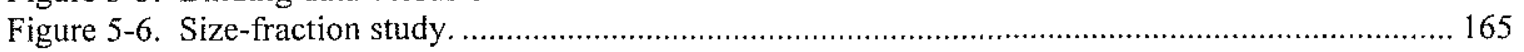




\section{TABLE OF TABLES}

\section{Chapter 2}

Table 2-1. Parameters used in estimates of uptake rates via diffusion and ingestion................................. 36

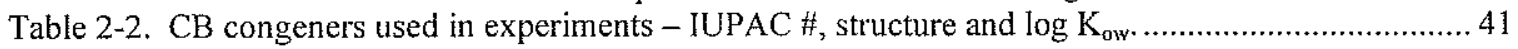

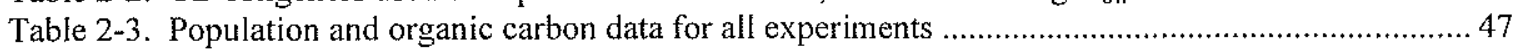

Table 2-4. Aqueous solubilities, concentrations and comparisons for all $\mathrm{CB}$ congeners used................... 49

\section{Chapter 3}

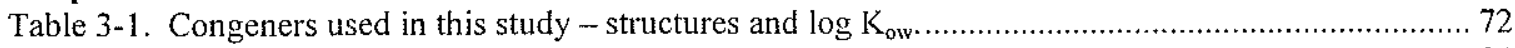

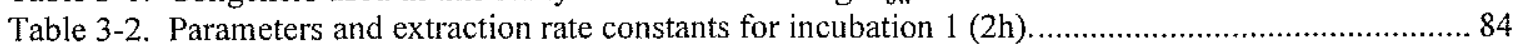

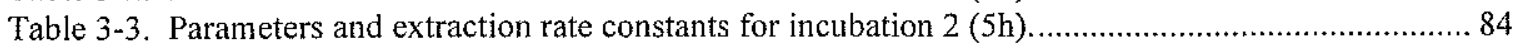

\section{Chapter 4}

Table 4-1. Summary of the characteristics of "grazer-enhanced" C/DOM in cited investigations............. 100

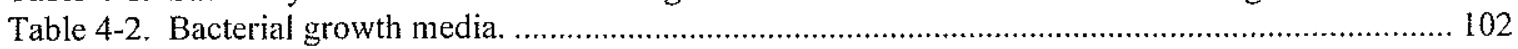

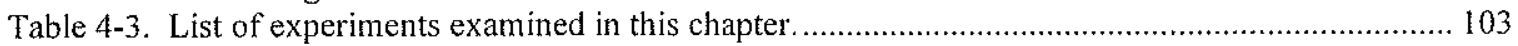

Table 4-4. Maximum ingestion rates and activities for protists studied. ……................................... 114

Table 4-5. Bulk and compositional lipid data for protozoan and bacterial samples. ........................... 116

Table 4-6. Measured surfactant activities in seawater, cultures and this study. .................................... 118

\section{Chapter 5}

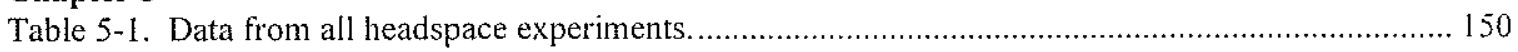

Table 5-2. Data from all headspace experiments - Uronema and H. halodurans................................... 151

Table 5-3. Comparison of this study's values for $\mathrm{K}_{\mathrm{DOC}}$ of seawater DOC with literature values.............. 154

Table 5-4. Lipid concentrations in seawater from different regimes compared to those in our study....... 156 


\section{Introduction}

\subsection{General justification for thesis}

Anthropogenic activities have introduced xenobiotic hydrophobic organic compounds into the natural environment. Many of these contaminants have a myriad of toxic and mutagenic effects and present a hazard to human and ecological health. One class of these pollutants is the polychlorinated biphenyls (PCBs). The structural foundation of all chlorobiphenyls (CBs) is the biphenyl ring system. Different congeners (isomers and homologues) are formed by attaching chlorine atoms to various positions on this ring system. Theoretically, there are 209 possible congeners, representing a range of one to ten substituted chlorine atoms. These compounds were synthesized primarily for use in the electrical industry as insulation for electrical transformers and capacitors (NRC, 1979). Before 1971, there was limited usage of these compounds as lubricants and de-dusting agents in other industries. In 1971, however, the production of PCBs was banned in the United States and their use was allowed only in closed systems, such as electrical transformers. Consequently, old CB-containing electrical equipment is the only source of PCBs still remaining in the United States and its disposal is strictly regulated. Aquatic (lacustrine, riverine and marine) sediments are the ultimate depository for PCBs in the United States. PCBs can be removed from the environment by anaerobic and aerobic microbial degradation as well as photolysis in the atmosphere. These processes occur on very long time scales and are subject to significant congener differences. PCBs are assumed, then, to be relatively inert to chemical or biological degradation on short time scales. As the industrial use of PCBs declines steadily, recycling processes at the sediment-water interface in lacustrine and marine environments will constitute the major source of PCBs to marine and fresh water systems (NRC, 1979). The controlling factors for recycling processes and their effect on $\mathrm{CB}$ speciation in contaminated sediments must be characterized to assess the extent to which PCBs are remobilized to the overlying water column.

The work presented in this thesis sought to characterize the impact of the microbial loop and specifically, protozoan grazers on the cycling of PCBs in marine 
systems. CB dynamics were examined in two-phase laboratory systems consisting of protozoa and bacteria. Chapters 2 and 3 evaluate the extent to which these experimental systems are in equilibrium within the relevant time scale for grazing processes (2-5 days). Chapter 4 examines the production and composition of "grazer-enhanced" dissolved organic material (DOM) in cultures of three protozoan species. Chapter 5 measures the affinity of this material for PCBs relative to bacterially-derived material and Vineyard Sound seawater DOM.

\subsection{Polychlorinated biphenyls (PCBs)}

Polychlorinated biphenyls are ubiquitous and persistent in the global environment. The residence time and distribution of specific chlorobiphenyl (CB) congeners are difficult to predict in aquatic systems due to the range of chemical properties within this class of compounds. For example, aqueous solubilities span five orders of magnitude (10-3 to 10-8 g/L - Mackay et al., 1980). Due to the dependence of many chemical properties on chemical structure, variations in aqueous solubility within the PCB class exist not only as a function of molecular weight but also as a function of the position of chlorine substitution. As a result of high hydrophobicity (i.e., low aqueous solubility), PCBs preferentially associate with organic-rich phases and consequently accumulate in the tissues of organisms. While they travel up the food chain, these compounds are biomagnified at each trophic level (Evans et al., 1991). At the higher trophic levels, the toxic effects of PCBs are subtle, resulting in reproductive disorders as well as behavioral development problems rather than acute sickness or death (NRC, 1979). Biodegradation of PCBs by bacteria is slow but present in aerobic environments (review - Furukawa, 1986). Field investigations have also observed degradation via reductive de-halogenation under anoxic conditions (Abramowicz, 1990; Bedard and May, 1996). Dehalogenation under anaerobic conditions removes chlorines from the meta and para positions preferentially. Degradation in the presence of oxygen occurs via the destruction of the biphenyl ring system and preferentially affects low-chlorinated congeners due to steric effects. 


\subsubsection{Current models of speciation}

Studies have shown that PCBs adsorbed onto abiotic particles or complexed by dissolved organic matter (DOM) are relatively unavailable to bacteria and phytoplankton in comparison to PCBs truly dissolved in the aqueous phase (reviews - Farrington, 1991; Mihelcic et al., 1993; Suffet et al., 1994). These studies have led to the hypothesis that the primary mechanism by which PCBs enter the aquatic food chain is via dissolved uptake by bacteria and phytoplankton. In natural aquatic systems, many factors can affect the relative fraction of dissolved PCBs, including DOM concentrations, particle flux to the sediment-water interface, percent organic carbon in particles and congener hydrophobicity.

The simplest model used to predict the dissolved concentration of PCBs and other hydrophobic organic compounds assumes that they exist in equilibrium between the aqueous and abiotic particulate phases (Karickhoff et al., 1979). This equilibrium partitioning can be represented mathematically by the following equation:

$$
\text { (1) } K_{p}=\frac{C_{p}}{C_{w}}
$$

where $\mathrm{C}_{\mathrm{p}}$ is the concentration in the particulate phase, $\mathrm{C}_{\mathrm{w}}$ is the concentration in the aqueous phase and $\mathrm{K}_{\mathrm{p}}$ is the non-dimensional proportionality constant, or the equilibrium partition coefficient. Organic carbon content and compound hydrophobicity appear to be the primary factors controlling the value of $K_{p}$. Therefore, $K_{p}$ is often normalized to the fraction of organic carbon $\left(\mathrm{f}_{\mathrm{oc}}\right)$ to produce a relatively constant partition coefficient, $\mathrm{K}_{\mathrm{oc}}$, that is applicable to sediments with $f_{o c}>0.001$ (Schwarzenbach and Westall, 1981). Increasing $\log \mathrm{K}_{\mathrm{oc}}$ is linearly related to increasing $\log \mathrm{K}_{\mathrm{ow}}$, the $n$-octanol-water partition coefficient (Karickhoff et al., 1979; Schwarzenbach and Westall, 1981; Schwarzenbach et al., 1993). Since $\mathrm{K}_{\mathrm{ow}}$ is a measure of a compound's hydrophobicity, a linear relationship between $\log \mathrm{K}_{\mathrm{ow}}$ and $\log \mathrm{K}_{\mathrm{oc}}$ implies that hydrophobic organic contaminants with high $\mathrm{K}_{\mathrm{ow}}$ values will preferentially partition into the organic-rich phases of the aquatic system. Other variables may also be important for controlling particle 
associations (for example - temperature and total suspended solids - Bergen et al., 1993; salinity - Means, 1995) but $\mathrm{K}_{\text {ow }}$ seems to be the most dominant factor (Schwarzenbach et al., 1993).

In this two-phase model, the hydrophobic, or particle-reactive, compound is assumed to be associated with natural organic matter on the surface of abiotic (i.e., nonliving) particles. However, further studies have shown that the measured dissolved concentrations of very hydrophobic contaminants, e.g., highly chlorinated PCBs (log Kow of 7.5-8 - Hawker and Connell, 1988), exceed their aqueous solubilities. Consequently, it is clear that the two-phase equilibrium model does not fully explain the partitioning behavior of hydrophobic organic contaminants observed in aquatic systems (Wijayaratne and Means, 1984; Gschwend and Wu, 1985; Baker et al., 1986; Brownawell and Farrington, 1986). The reason for this lies in the difficulty of determining analytically the true aqueous concentration, $\mathrm{C}_{\mathrm{w}}$, due to interferences by macromolecular or colloidal material within the aqueous phase.

Separation methods using filters with nominal pore sizes of $0.2-0.7 \mu \mathrm{m}$ or centrifugation cannot fully separate this macromolecular or colloidal material from the aqueous phase. In the absence of phase separation methods that completely remove these high molecular weight materials, a three phase model must be used to describe equilibrium speciation. In this model, the phases are defined as particulate material, colloidal material and the aqueous phase (Gschwend and $\mathrm{Wu}, 1985$; Brownawell and Farrington, 1986):

$$
\text { (2) } K_{p}=\frac{C_{p}}{C_{w}+C_{c}}
$$

where $C_{c}$ is the colloidal concentration. Studies by Gschwend and Wu (1985), Brownawell and Farrington (1986) and others show that the three phase model more accurately explains experimental data. The dependence of $\mathrm{K}_{\mathrm{p}}$ on organic carbon content and compound hydrophobicity in this model is assumed to be similar to that of $K_{p}$ in the two-phase model described above. However, it is possible that colloidal material has a different composition than the larger particulate material (e.g., Taylor et al., 1985). As a 
result, two different $\mathrm{K}_{\mathrm{p}}$ 's should be used to explain fully the partitioning behavior of PCBs (Gschwend and $\mathrm{Wu}, 1985$ ). Nonetheless, as a first approximation, the three phase model fits experimental data quite well (Brownawell and Farrington, 1986).

The above two models assume equilibrium partitioning of PCBs between abiotic (non-living) particles and the surrounding aqueous phase. In a laboratory study testing this hypothesis, Wu and Gschwend (1986) found that equilibrium between aqueous PCBs and sediment grains was attained within two hours. This and other sorption kinetics studies (Tye et al., 1996) test only the equilibrium partitioning predicted by the physicalchemical model above; they do not examine the kinetics-limited uptake processes that can occur in unicellular organisms. Stange and Swackhamer (1994) and Swackhamer and Stange (1993) have studied the kinetics of uptake of PCBs in phytoplankton. Their results indicated that $\mathrm{CB}$ concentrations in an organism and the surrounding aqueous phase did not reach equilibrium values until 10 days after PCBs were added to the system. Instead of the instantaneous partitioning assumed by the equilibrium model, they observed a two-stage process consisting of rapid uptake for 1-2 hours and slow equilibration with cellular carbon for the remainder of the experiment. These investigators proposed that the initial uptake was equilibration of the cellular surface with the aqueous $\mathrm{CB}$ concentration. Subsequent transport (active and/or passive) across the cellular membrane into the cell interior was a possible explanation for the second stage of CB uptake (Stange and Swackhamer, 1994). The kinetics of this two-stage process were dependent on the surface area-to-volume ratios and growth rate of the phytoplankton (Skoglund et al., 1996). These studies have only been performed with phytoplankton (size $10-102 \mu \mathrm{m}$ ). Whether these results can be extended to larger or smaller unicellular organisms was not ascertained. However, given these studies, it is possible that the kinetics of uptake of PCBs by organisms will not be as rapid as the diffusive equilibration proposed by the abiotic models described above. These models of PCB speciation can be integrated into a conceptual model shown in Figure 1-1. 


\subsubsection{Effect of structure and chemical composition on $C B$ sorption to organic matter}

PCBs will readily partition into organic matrices occurring in aquatic systems because of low aqueous solubilities and concomitant high hydrophobicities. The sorption of PCBs to inorganic particulate material such as oxides and to colloidal or dissolved organic matter is sensitive to the influences of structure and chemical composition. While surface-adsorption of PCBs onto inorganic particulate oxides will occur, incorporation into the matrix of the particle and association with organic matter is a function of the diffusion of PCBs into the inner oxide and the affinity of the associated organic matter for PCBs (Wu and Gschwend, 1988).

As discussed above, colloidal/dissolved organic matter has been shown to influence the residence time of PCBs by "sequestering" PCBs within the suspended pool. The extent of this influence will vary depending on the size, conformation, and chemical composition of the material acting as substrate. First, a microenvironment conducive to PCB dissolution must be present either within the structure of the material (Gustafsson and Gschwend, 1997) or in a pocket along the surface (partial desolvation from $\mathrm{H}_{2} \mathrm{O}$ ). Therefore, the material must be large enough to be able to fold and aggregate such that an inner hydrophobic environment can form. Many biomolecules are large enough to achieve such a conformation (Benner et al., 1992; Aluwihare et al., 1997). In addition, the material must have some affinity for PCBs. Some studies have shown decreasing sorption with increasing organic phase polarity (increasing $\mathrm{C} / \mathrm{O}$ ratios - Chiou et al., 1986; Gauthier et al., 1987). For example, compounds such as cellulose are poorer sorbents for PCBs than lipid-rich molecules (Garbarini and Lion, 1986). The relative partition coefficients of carbohydrates and lipids are so divergent that large concentrations of carbohydrates are necessary to "sequester" as many PCBs as very small concentrations of lipids. Therefore, small increases in lipid-rich colloidal material will have a large impact on the speciation of PCBs within a particular system, whereas the effect of small increases in carbohydrate material would not be detected. 


\subsection{Protozoa}

\subsubsection{Microbial loop}

The concept of a microbial loop was first introduced by Pomeroy (1974) and further formalized by Azam et al. (1983). The microbial loop is a complex food web consisting of bacteria, phytoplankton and micrograzers such as nanoflagellates and ciliates. The ability of protozoan grazers to recycle and remineralize both organic matter and inorganic nutrients has been the subject of numerous laboratory and field-based studies (e.g., Sherr et al., 1982; Caron et al., 1985; Goldman et al., 1985; Andersen et al., 1986; Goldman et al., 1987; Caron et al., 1988; Sherr and Sherr, 1988; Caron et al., 1991; Sherr and Sherr, 1994; Barbeau et al., 1996; Barbeau et al., submitted). A particularly exhaustive study (Caron et al., 1985; Goldman et al., 1985; Andersen et al., 1986; Goldman et al., 1987) showed that protozoan grazers excrete inorganic nutrients such as nitrate and phosphate as well as dissolved organic carbon (model in Figure 1-2). The concept of this dynamic recycling process has implications for the study of organic carbon remineralization in all regimes, including oligotrophic waters such as the Sargasso Sea, coastal areas such as estuaries, and the sediment-water interface in both lacustrine and marine systems. Now that the presence of this loop and cognizance of its importance has been firmly established, studies are focusing on the time scales of the grazing processes (Sherr et al., 1987; Sherr et al., 1989; Caron et al., 1991), and the nature of the excretion products (Nagata and Kirchman, 1992b; Tranvik et al., 1993; Tranvik, 1994).

\subsubsection{Roles of protozoa in the microbial loop}

\subsubsection{Particle size spectrum}

Phagotrophic flagellate and ciliate protists are able to remineralize a significant fraction of ingested bacterial and algal biomass ( $>50 \%$ - Fenchel, 1987). To a first approximation, nano-protists discriminate between particles primarily on the basis of size, consuming particles in the $0.1-1.0 \mu \mathrm{m}$ range. The degree of prey selectivity is a function of the feeding mechanism of the protist. Filter feeders exhibit little selectivity and ingest particles within a particular size range. Raptorial feeders can be expected to 
exhibit greater food selectivity because they ingest particles one-by-one. Prey particles can include unicellular organisms like bacteria and algae as well as small detrital particles or particles associated with larger particles.

After engulfing their prey, protists digest their food with a dynamic chemical process inside the digestive vacuole (Figure 1-3). Initially, particles are subjected to a drop in $\mathrm{pH}$ to levels of 1.4-3 as well as intense enzymatic activity. The low $\mathrm{pH}$ values and digestive enzymes cause prey cell lysis. The $\mathrm{pH}$ increases during the second stage of digestion as the vacuole fuses with the lysosomal membrane and the waste is excreted (Fok et al., 1982). This digestive process lasts approximately 20 to $60 \mathrm{~min}$ (Fok et al., 1982; Fenchel, 1987). Waste products can range from large aggregates of excreted material $(\geq 5.0 \mu \mathrm{m})$ to colloidal particles $(<0.2 \mu \mathrm{m}$ - operational definition for the purposes of this thesis) to dissolved materials. Heterotrophic nanoflagellates can clear the prey from a volume of water $10^{5}$ times their cell volume within an hour. Given the breadth of the size range represented by the waste particles in comparison to the ingested particles as well as the magnitude of the clearance rate, it is clear that protozoans have an impact on the distribution of the particle size spectrum.

Carbon dynamics in laboratory cultures showed two extremes of particle size spectrum effects (Barbeau, 1998). In an experiment with a nanoflagellate, Cafeteria sp., dissolved organic carbon (DOC) concentrations increased in the flagellate culture relative to the control during protozoan exponential growth, presumably as a result of remineralization of bacterial biomass. In an experiment with another flagellate, Paraphysomonas imperforata, bacterial organic carbon was repackaged into larger sized particles $(>5.0 \mu \mathrm{m})$ instead of entering the dissolved pool. These two experiments represent the extremes of effects that grazing activity can have on particle size distributions. Cafeteria sp. generated large quantities of dissolved material whereas $P$. imperforata repackaged bacterial material into larger particles that sank out of the culture medium quickly.

These results were extended to particulate (colloidal) metal oxides in recent laboratory (Barbeau et al., 1996) and field studies (Barbeau and Moffett, submitted). In 
these studies, iron hydroxides, impregnated with a chemically inert tracer, were fed to protozoa in laboratory culture and to natural assemblages collected from Vineyard Sound, MA. The accumulation of the inert tracer in the dissolved fraction $(<3500$ molecular weight cutoff) was linearly related to the amount of dissolution of the hydroxides and served as a measure of protozoan grazing. These results showed that protozoan grazers in laboratory culture and in the $2-20 \mu \mathrm{m}$ size fraction of Vineyard Sound seawater were capable of dissolving colloidal iron hydroxides. The redox state of the excreted Fe was hypothesized to be $\mathrm{Fe}^{+2}$. Experiments with iron-limited diatom cultures showed increased diatom growth in the presence of grazers and colloidal iron-hydroxide coated bacteria (Barbeau et al., 1996).

\subsubsection{Chemical composition of particulate and dissolved organic matter}

Recent work by Nagata and Kirchman (1992b) and Tranvik (1994), has shown that protozoan grazers may represent one source of colloidal material. In laboratory culture, Nagata and Kirchman (1992b) found that protozoan grazers excreted significant amounts of macromolecular material (5 to $57 \%$ of labeled DOM) as defined by a cold trichloroacetic acid (TCA) precipitation. They proposed that this high molecular weight, or colloidal, material was coated with a layer of phospholipids derived from bacterial prey. Tranvik also noted an enhancement of colloidal material $(0.02 \mu \mathrm{m}-0.2 \mu \mathrm{m})$ in grazing cultures. Radiolabeled prey studies showed that this material was most likely derived from internal cellular components rather than cell wall material. Differences in organic carbon composition may affect affinity of DOM for particle-reactive compounds and elements such as PCBs and Th. Experiments with ${ }^{234} \mathrm{Th}$-labeled prey showed an increase in Th-to-C ratios in the particulate organic size fractions $(0.2-1.0 \mu \mathrm{m}, 1.0-5.0 \mu \mathrm{m}$,

and $>5.0 \mu \mathrm{m}$ ), suggesting either increased particle surface area or higher affinity for ${ }^{234} \mathrm{Th}$ over DOM in bacterial controls (Barbeau et al., submitted).

In marine environments, the composition of particles along the size spectrum can vary tremendously. The relative abundance of inorganic and organic components is a function of depth in the water column due to preferential degradation of organic-rich 
particles. In addition, there are spatial variations in the composition of the particle size spectrum due to nutrient variability (i.e., changes in primary production) as well as atmospheric deposition patterns (i.e., changes in inorganic mineral composition). As an example, Sackett (1978) showed that particles in the upper $10 \mathrm{~m}$ in the Gulf of Mexico were composed primarily of organic constituents $(85 \%)$. This composition was altered dramatically by biological degradation within the water column, resulting in the deposition of primarily inorganic particles $(66 \%)$ at the sediment-water interface. Coastal studies have shown that organic carbon composition is often $1-5 \%$ in fine-grained nearshore sediments, consistent with the data of Sackett (1978). Since organic-rich particles constitute such a small fraction of total material at the sediment-water interface, any additional source of organic material in this system could affect speciation of compounds governed by organic carbon composition.

\subsection{Protozoa and contaminants}

\subsubsection{Presence of protists at contaminated sites}

The presence of protists has been noted at a number of contaminated sites (Pratt and Cairns, 1985; Shen et al., 1986; Madsen et al., 1991; Sinclair et al., 1993; Harvey et $a l ., 1995)$. High concentrations of nutrients and organic matter in contaminated areas and sewage disposal sites support dense bacterial populations and thus active protozoan assemblages. The interactions between bacterial and protozoan populations can have a positive impact on the extent of bio-degradation occurring at these sites. For example, Sinclair et al. (1993) investigated a groundwater site contaminated with jet fuel. High biodegradation rates (by bacteria) occurred in areas of high jet fuel concentrations. However, bacterial populations at the contaminated site were comparable in density to those at an uncontaminated control site, implying that elevated biodegradation rates could not be explained simply by higher bacterial concentrations. In contrast, protozoan populations were significantly elevated at the jet fuel site (Figure 1-4). Sinclair et al. (1993) concluded that protozoa were actively grazing the bacteria, keeping them growing at exponential rates which in turn raised biodegradation rates. 


\subsubsection{Field studies suggesting importance of microbial loop}

Recent field studies (Lake Superior - Baker et al., 1991; Mediterranean Sea Lipiatou et al., 1993; Esthwaite Water - Sanders et al., 1996) highlight the need for the integration of both the biological (Skoglund et al., 1996) and physico-chemical (Wu and Gschwend, 1986) models of $\mathrm{CB}$ speciation. The equilibrium partitioning models predict that PCBs will absorb into abiotic particles during settling. However, it has been noted that downward CB fluxes to the sediment, when calculated using sediment traps, are greater (100X) than those calculated using accumulation rates measured in sediment cores (Figure 1-5). The data suggested that $85-90 \%$ of the PCBs deposited onto the sediment-water interface were recycled and returned to the water column (Baker $e t a l$., 1991). These studies are based on material collected in sediment traps suspended within the lake water column. Concerns have been raised regarding the collection efficiencies and sample integrity of the collected materials by many authors (e.g., Lee et al., 1992). In the Baker et al. study (1991), the traps were poisoned with chloroform. This poison could preferentially extract hydrophobic compounds, including contaminants such as PCBs, from the water (Gundersen and Wassmann, 1990). The second study, performed by Sanders et al. (1996), used an inorganic poison $\left(\mathrm{HgI}_{2}\right.$ - mercuric red) in the sediment traps. The results from these studies should be viewed critically given the above concerns. Even so, the results point to efficient recycling processes occurring at the sediment-water interface. If true, contaminated sediments can be the largest source of CBs to the water column (Sanders et al., 1996).

Review of these data suggested that these recycling processes could be mediated by micro-organisms inhabiting the sediment-water interface. The particles ingested by protozoa $(0.1-1.0 \mu \mathrm{m})$ constitute a major pool of surface area in aquatic systems and thus a potentially significant reservoir of surface-active contaminants (based on particle size class distributions and surface area:volume ratios). The ability of protists to alter both the size spectrum and chemical composition of particles at the sediment-water interface 
suggests that grazing processes can affect $\mathrm{CB}$ speciation in this milieu and subsequently influence the residence time of $\mathrm{PCBs}$ within an aqueous system.

\subsection{Thesis background, development, and summary}

\subsubsection{Model system}

This thesis is based on a series of laboratory experiments designed to elucidate various aspects of the microbial loop and its influence on $\mathrm{CB}$ speciation in regimes with an active microbial loop. To this end, a two-phase system was employed in all experiments. This system consisted of predator and prey suspended in sterile Vineyard Sound seawater. Three different protozoan species were used as predators - a ciliate, Uronema sp. (clone: BBCil), and two flagellates, Cafeteria sp. (clone: Cflag) and Paraphysomonas imperforata (clone VS1). The same bacterium, Halomonas halodurans, was used as prey in all experiments. All organisms were obtained from the collection of D. Caron, University of Southern California, CA. Chloro-biphenyls (CBs) were added to all experiments and their dynamics monitored. These experiments contained three pools of organic matter within the aqueous phase - colloidal/dissolved organic matter (C/DOM - also referred to as dissolved organic carbon or DOC), bacterial cells, and protist cells. The PCBs added to the system interacted and associated with each of these three pools according to physico-chemical properties of the organic matter. This thesis was concerned with the equilibrium $\mathrm{CB}$ concentrations and residence times within each of the organic pools mentioned as well as with changes within the C/DOM pool and the consequences for $\mathrm{CB}$ speciation. The end goal of the thesis was to understand (and potentially predict) the effect of protozoan grazers on the cycling of PCBs. By extension, these results can be used to estimate the release of PCBs from sediments at contaminated sites.

\subsubsection{Equilibration within organic phases of model system}

The first section of the thesis was concerned with the timing of $\mathrm{CB}$ uptake into protozoa. The production of organic matter by grazing protists occurs on the time scale 
of hours to days (Caron et al., 1985; Barbeau, 1998). CB equilibration with unicellular protists needs to occur much faster than these processes in order to determine accurately the fluxes of PCBs among the different organic pools present. Since ingestion of prey is intimately tied to the production of organic matter, uptake of PCBs by ingestion of contaminated prey is expected to occur on time scales of hours. A theoretical calculation predicted that the alternate method of $\mathrm{CB}$ uptake, diffusion, should be faster than ingestion of contaminated prey by a factor of 1000 . Experimental verification of this prediction was the goal of Chapter 2 .

Once it was established that PCBs could be taken up by protists on minute time scales, the rate constants for loss of CBs from bacteria and disassociation of CB-DOC complexes were examined to ensure that they were fast enough to supply the diffusive uptake by protozoa. A calculation in Chapter 2 suggested that the bacterial loss rate was comparable to protozoan uptake rates. The role of $\mathrm{CB}-\mathrm{DOC}$ complexes in the diffusive process is the subject of Chapter 3. Increased $\mathrm{CB}$ uptake from the ingestion of CB-DOC complexes did not increase $\mathrm{CB}$ ingestion rates enough to out-compete diffusion. In fact, diffusion of CB-DOC complexes to the surface of the protozoan may have increased the diffusion uptake rate.

\subsubsection{Production of material by protists and its affinity for PCBs}

Once it was clear that protozoa, bacteria and DOC equilibrated with the added PCBs within minutes, the effect of protozoan grazing on $\mathrm{CB}$ speciation could be examined. DOC dynamics were monitored in protozoan cultures and compared to bacterial controls. Specific components of bulk DOC were examined, such as surface-active material and lipopolysaccharides (Chapter 4). Production rates of these materials and ingestion rates by protozoa were compared and the influence of protozoan species and prey growth substrate was considered. The affinity of this "grazer-enhanced" DOM for a radiolabeled $\mathrm{CB}$ congener $\left(\left[{ }^{14} \mathrm{C}\right]-3,3^{\prime}, 4,4^{\prime}\right.$-tetrachlorinated biphenyl or $\left.\left[{ }^{14} \mathrm{C}\right]-\mathrm{TCB}\right)$ was determined in Chapter 5 using a headspace partitioning method. Culture filtrates $(<0.2 \mu \mathrm{m})$ were 
inoculated with $\left[{ }^{14} \mathrm{C}\right]-\mathrm{TCB}$ and the affinity of DOM was compared among protozoan cultures, bacterial controls and Vineyard Sound seawater.

The results from the four data chapters were summarized in Chapter 6 and used to synthesize a picture of $\mathrm{CB}$ dynamics in the microbial loop. The effect of protozoan grazing on the release of PCBs from contaminated sediments was also discussed. 


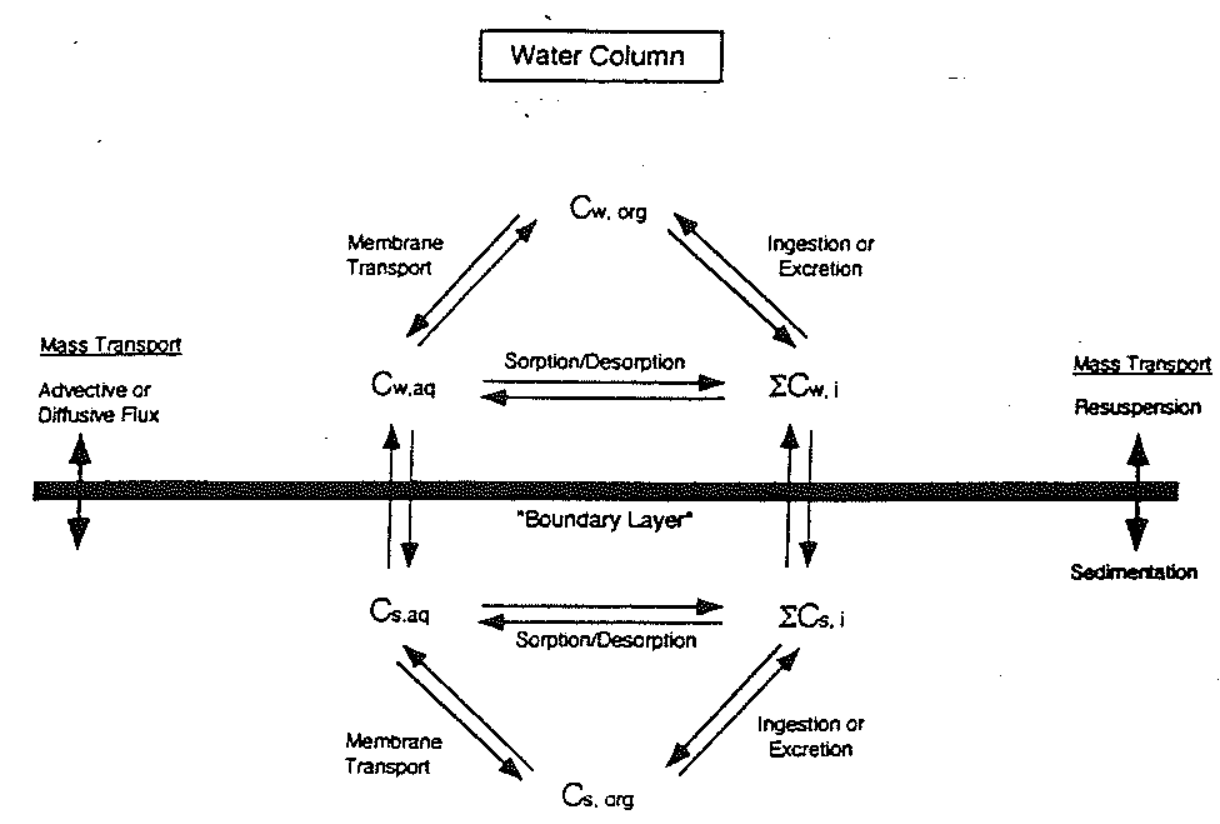

Sediment

Figure 1-1. Conceptual model of $\mathrm{CB}$ speciation in aquatic systems.

The above figure is taken from Suffet et al. (1994). The subscripts refer to (1) the environmental phase [water (w) or sediment (s)] and (2) the phase within that compartment [aqueous (aq), organism (org), sorbing phase (i)]. The kinetic barriers are not shown here but will retard the uptake of contaminants into the biota (organisms) or the sorbing phase.

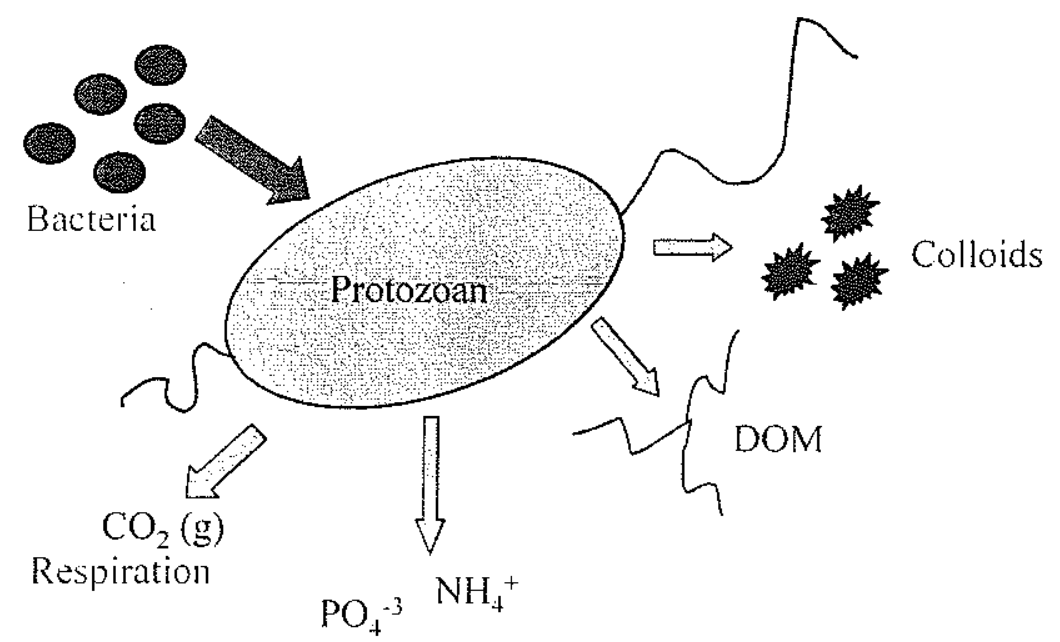

Inorganic nutrients

Figure 1-2. Carbon and nutrient cycles in model system containing protozoa and bacterial prey.

Carbon, nitrogen and phosphorus contained in bacterial biomass are ingested by phagotrophic protozoa. Inorganic carbon $\left(\mathrm{CO}_{2}(\mathrm{~g})\right)$ and nutrients $\left(\mathrm{NH}_{4}{ }^{+}, \mathrm{PO}_{4}{ }^{-3}\right)$ are respired and remineralized. Organic material (both dissolved and colloidal) is also excreted after digestion. The composition of the organic material varies as a function of feeding mechanism and digestive assimilation efficiency. 


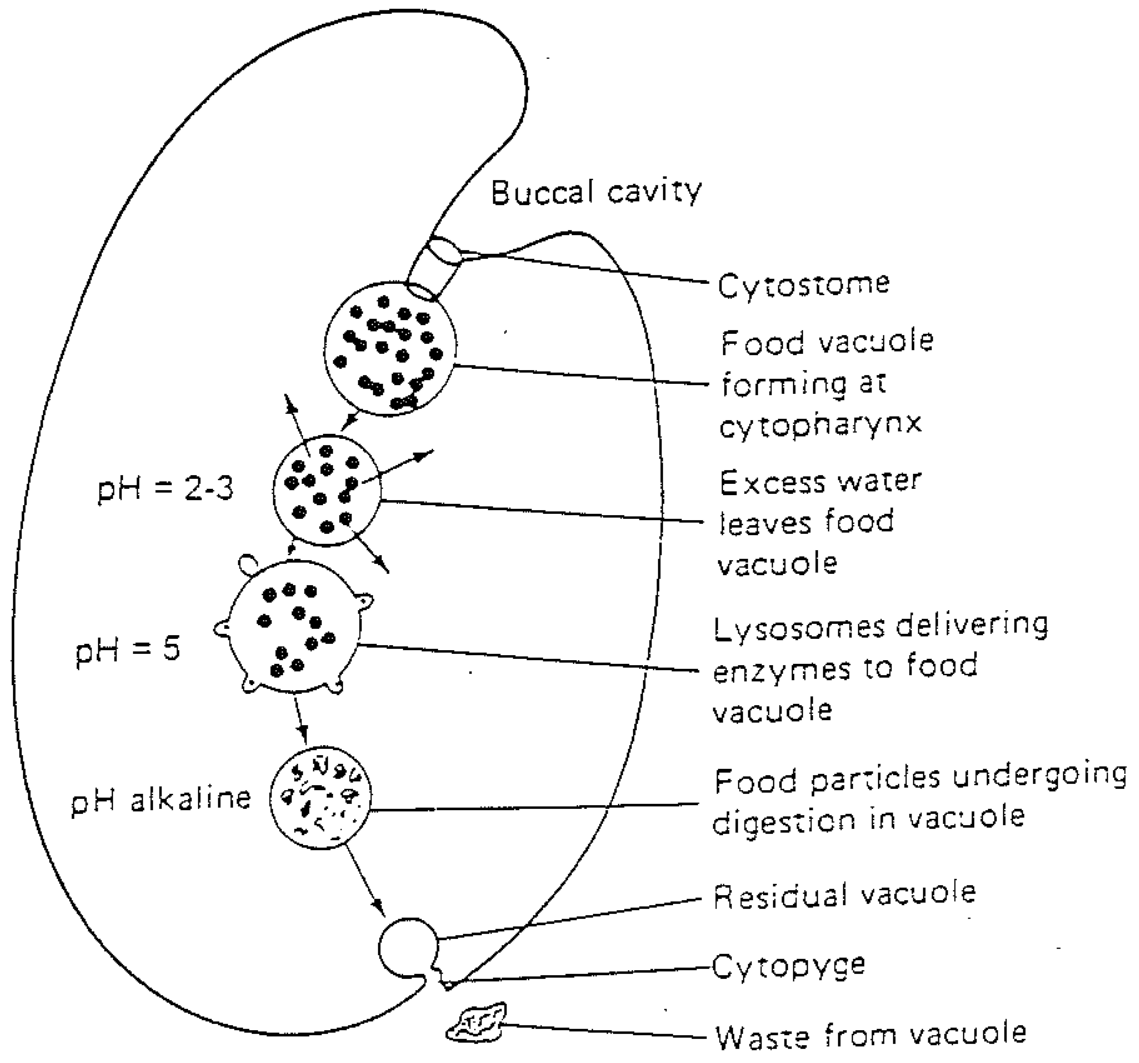

Figure 1-3. Schematic of protozoan digestive system.

From Adey \& Loveland (1991). 


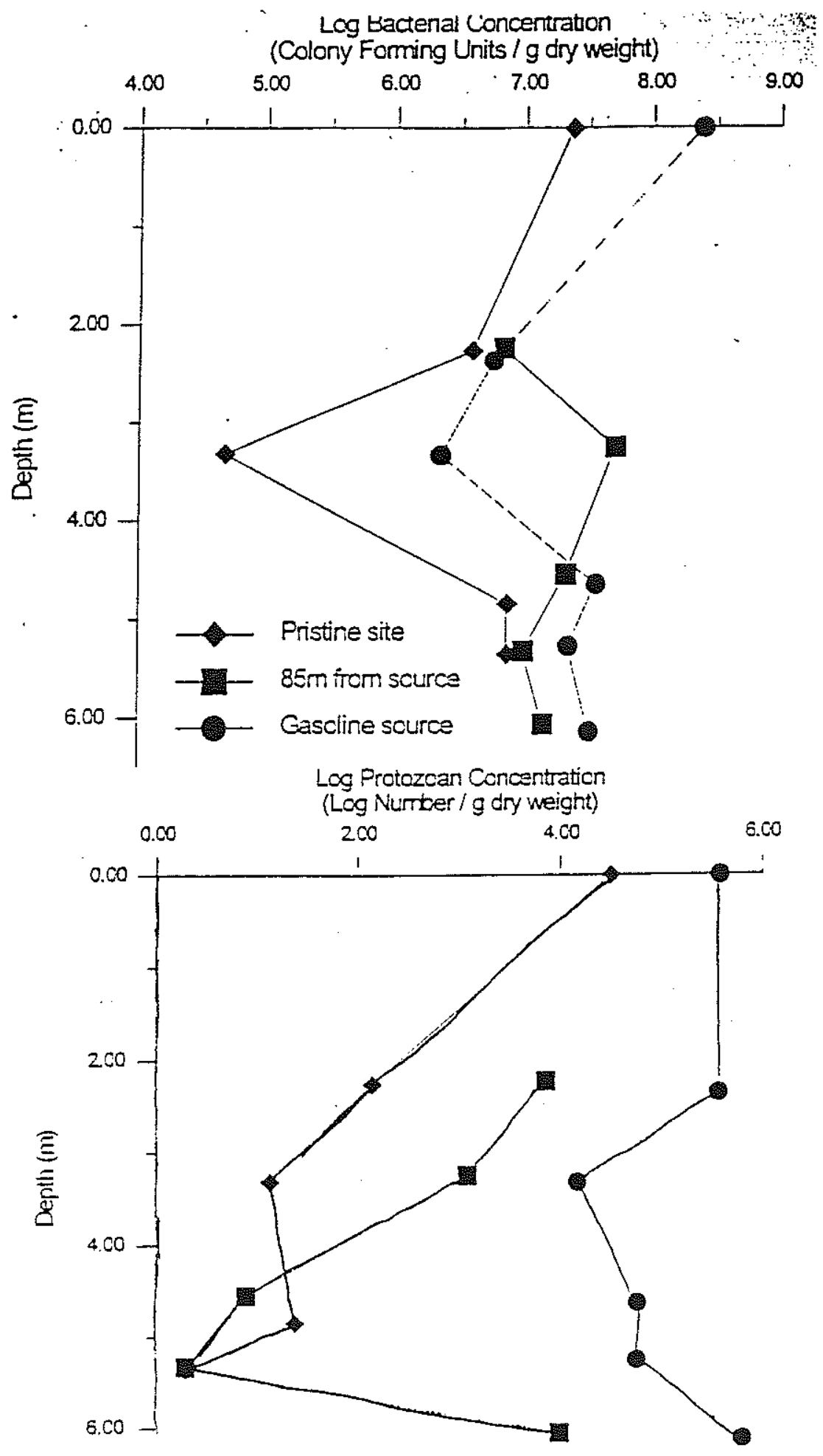

Figure 1-4. Protozoan and bacterial dynamics at a jet-tuel contaminated site.

Data taken from Sinclair et al. (1993). The $3.5-4.5 \mathrm{~m}$ range for the site $85 \mathrm{~m}$ from fuel spill has low $\mathrm{O}_{2}$ and high fuel concentrations. 


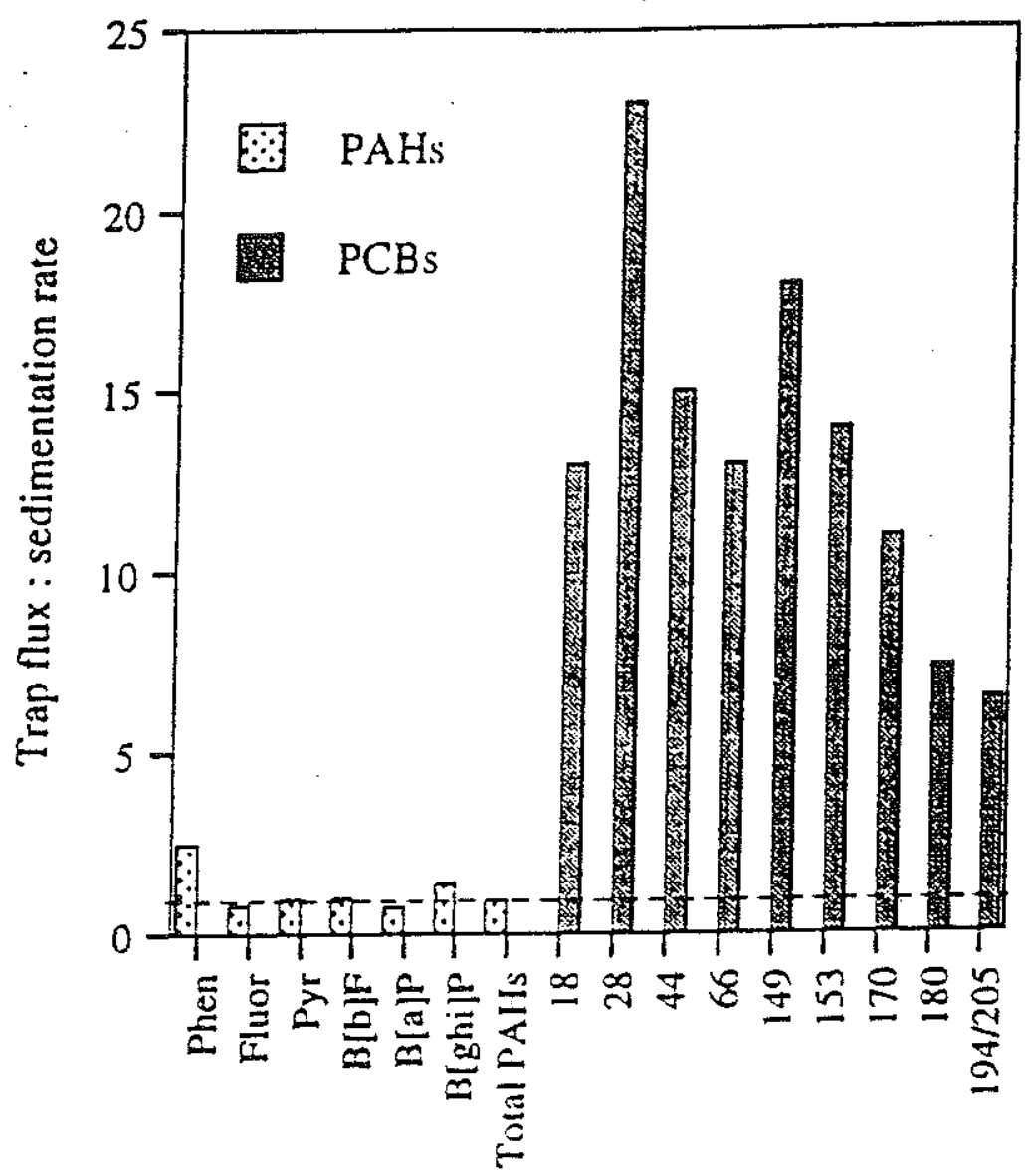

Figure 1-5. Ratio of depositional fluxes to sedimentation rates for selected $\mathrm{CB}$ congeners and polyaromatic hydrocarbons (PAHs).

Figure 5 from Sanders et al. (1996). Depositional fluxes measured in $12.5 \mathrm{~m}$ trap of small rural lake. Dashed line represents ratio of $1: 1$. 


\section{The importance of passive diffusion in the uptake of PCBs by phagotrophic protozoa}

\section{Submitted to Applied and Environmental Microbiology}

\subsection{Abstract}

Phagotrophic protozoan grazers represent an intersection between two methods of introduction of polychlorinated biphenyls (PCBs) into marine organisms - diffusion through surface membranes and ingestion of contaminated prey. This study compares the relative importance of these two processes in the overall uptake of PCBs by unicellular protists. Uptake rates and steady-state concentrations were compared in laboratory cultures of grazing and non-grazing protozoa. These experiments were conducted with a $10 \mu \mathrm{m}$ marine scuticociliate (Uronema sp.), bacterial prey (Halomonas halodurans), and a suite of 21 chlorobiphenyl (CB) congeners spanning a range of aqueous solubilities. The dominant pathway of $\mathrm{CB}$ uptake by both grazing and non-grazing protozoa was diffusion. Bioconcentration factors (BCFs) were equivalent in grazing and non-grazing protozoa for all congeners studied. Rate constants for uptake into and loss from the protozoan cell were independently determined using ${ }^{14} \mathrm{C}-3,3^{\prime}, 4,4^{\prime}$-tetrachlorobiphenyl (IUPAC \#77). The protozoan first-order uptake rate constant and second-order loss rate constant were $0.38 \pm 0.03 \mathrm{~min}^{-1}$ and $(1.1 \pm 0.1) \times 10^{-5}(\mathrm{~g} \text { org C})^{-1} \mathrm{~min}^{-1}$, respectively. Magnitudes of the uptake and loss processes were calculated and compared using a numerical model. The model result was consistent with data from the bioaccumulation experiment and supported the hypothesis that diffusive uptake is faster than ingestive uptake in phagotrophic unicellular protozoa.

\subsection{Introduction}

\subsubsection{Polychlorinated biphenyls.}

Polychlorinated biphenyls $(\mathrm{PCBs})$ are persistent organic pollutants ubiquitous in the global environment which have been shown to have adverse effects on the health of many aquatic organisms (Burreau et al., 1997; Means and McElroy, 1997; Munns et al., 
1997; Schweitzer et al., 1997; Mayer et al., 1998). They accumulate in the lipid-rich compartments of organisms due to high hydrophobicity. Whereas biotransformation of biphenyls with vicinal hydrogen atoms has been observed in several species of marine organisms (Kannan et al., 1995), the lack of vicinal H-atoms inhibits degradation resulting in efficient transfer of many chlorinated biphenyls between trophic levels (Connolly, 1991; Morrison et al., 1997).

\subsubsection{Introduction into organisms.}

Polychlorinated biphenyls enter aquatic organisms in two ways: diffusion through outer membranes and ingestion of chlorobiphenyl (CB)-contaminated detritus or prey. Diffusion of PCBs through the cellular membrane is the only uptake pathway available for non-phagocytotic unicellular organisms such as phytoplankton. In this process, PCBs associate with outer membranes and rapidly diffuse into the phospholipid bilayer surrounding the cytoplasm (Dulfer et al., 1996; Dulfer et al., 1998). For large organisms such as fish and marine mammals, PCBs accumulate through ingestion (Connolly, 1991; Campfens and Mackay, 1997; Kucklick and Baker, 1998). PCBs pass through the walls of the digestive system, enter the bloodstream and are subsequently carried to lipid-rich tissues. Uptake via gill and dermal exposure has been suggested to play a minor role in the overall uptake of PCBs by fish and other (large) marine organisms (Rubinstein et al., 1984; Connolly, 1991; Morrison et al., 1997).

This study focuses on $\mathrm{CB}$ uptake in unicellular phagotrophic protozoa, or nanozooplankton. These heterotrophic organisms feed primarily on particulate material in the $0.1-1.0 \mu \mathrm{m}$ size range, yet are comparable in size to the phytoplankton studied by other investigators (Swackhamer and Skoglund, 1993) and should accumulate PCBs by diffusion. Thus, these organisms offer a unique opportunity to study the relative rates of diffusive and ingested uptake.

Outside the protozoan cell, $\mathrm{CB}$ speciation is determined by the nature and concentration of organic carbon in both the dissolved and particulate pools. (Schwarzenbach et al., 1993). Studies have shown that PCBs absorbed within abiotic 
particles or associated with dissolved organic material (DOM) are relatively unavailable for biological uptake in comparison to PCBs truly dissolved in the aqueous phase (reviews - Farrington, 1991; Mihelcic et al., 1993). Dissolved PCBs enter the cell by diffusing through the cellular membrane. This process occurs as a series of steps. First, the dissolved PCBs diffuse across the unstirred water boundary layer to associate with phospholipids, extracellular proteins and, to a lesser extent, polysaccharides on the cell surface. Next, the chlorobiphenyl is transported into or through the phospholipid bilayer either via diffusion through the hydrophobic center of the membrane or through channels formed by channel and transmembrane proteins (Alberts et al., 1983).

Because the diffusion coefficient of a compound through any medium depends on its molecular size and structure, uptake via diffusion can potentially discriminate against large or bulky congeners. Ingestion of bacterial prey, alternatively, is less likely to fractionate compounds based on size or structure (provided most of the food is metabolized) because the chemical and temporal dynamics differ greatly inside the microenvironment of the protozoan food vacuole. Once ingested, all PCBs associated with bacteria are considered part of the protozoan cell because the food vacuole cannot be analyzed separately. For these reasons, the incorporation efficiency of PCBs should be close to $100 \%$ and no fractionation among congeners should occur. The concentration of PCBs in the bacteria is an important parameter in determining the CB uptake via ingestion. The size (i.e., surface area-to-volume ratio) and composition of the bacterial cell will play a role in determining this concentration.

Ingestion of prey begins with the invagination of the cellular membrane, encapsulating a parcel of surrounding water containing both free and complexed PCBs. The cellular membranes of ingested bacteria are disrupted by a dynamic digestive cycle ( $\mathrm{pH}$ drops to 2-3, the food vacuole fuses with lysozymes, $\mathrm{pH}$ rises back to alkaline levels and intense enzymatic activity ensues - Fok et al., 1982). Nutrients are transported across vacuole walls and waste material is released. Waste material can include bacterial cell membrane fragments, potentially in micellar form (Nagata and Kirchman, 1992b). The vacuole membrane is then reincorporated into the outer cellular membrane. Once inside 
the cell, the incorporated PCBs partition among the cellular components and the highest concentrations occur in organic-rich lipid storage compartments (Dulfer et al., 1998).

The difference in the two possible uptake pathways is a kinetic one. The steadystate $\mathrm{CB}$ concentration of the protozoan cell is the equilibrium value predicted by $\mathrm{K}_{\mathrm{ow}}$ (the $n$-octanol/water partition coefficient) of the $\mathrm{CB}$ congener and determined by the relative size and composition of the organic carbon pools in the system. The uptake pathway should not affect the final concentration in the protozoan cell, though it does affect the time needed to achieve this equilibrium value (Connell, 1989; Connolly, 1991).

\subsubsection{Initial calculation.}

An initial calculation for 3,3',4,4'-tetrachlorobiphenyl (IUPAC \#77) shows that the diffusion uptake pathway is faster than the ingested uptake pathway. This calculation is normalized to a single protozoan cell and assumes that the rate-limiting step of diffusive uptake is transfer across the lipid membrane. The other slow step in this process is diffusion across the unstirred water boundary layer surrounding the cell. However, with this set of organisms, the width of the boundary layer is difficult to estimate. The cellular surface is covered with cilia whose motion stirs the surrounding water and enables the ciliate to move through the water. The constant movement of these cilia will lower the thickness of the unstirred water boundary layer.

The calculation for diffusive uptake is based on Fickian diffusion described by:

$$
\text { (1) Flux }=D_{m} \frac{\Delta C}{z}=D_{m} \frac{C_{m u t}-C_{i m}}{z}
$$

where $D_{m}$ is the molecular diffusion coefficient through a medium, in this case, the cellular membrane, $\mathrm{C}_{\text {out }}$ and $\mathrm{C}_{\text {in }}$ are the concentrations of the compound ( $\mathrm{CB}$ congener) at the outer and inner cellular membrane, respectively and $\Delta z$ is the thickness of the lipid membrane. Two simplifying assumptions were made in this calculation. First, the outer membrane concentration, $\mathrm{C}_{\text {out }}$, was assumed to be equivalent to the concentration of the compound adsorbed on the cell surface, or $\mathrm{K}_{\mathrm{lw}}{ }^{*}[\mathrm{CB}]_{\mathrm{d}}$, where $\mathrm{K}_{\mathrm{lw}}$ is the lipid-water 
partition coefficient and $[\mathrm{CB}]_{\mathrm{d}}$ is the dissolved $\mathrm{CB}$ concentration. Second, the inner membrane concentration was assumed to be zero.

Therefore, the rate of uptake via diffusion is defined as the flux through the phospholipid membrane multiplied by the surface area of the protozoan and expressed by the following equation:

$$
\text { (2) }\left(\frac{d[C B]_{p r o y}}{d t}\right)_{\text {diff }}=F l u x * S A_{p r o t}=\frac{D_{m} K_{l w} S A_{p r o t}}{\Delta z}[C B]_{d r}
$$

where $[\mathrm{CB}]_{\text {prot }}$ and $[\mathrm{CB}]_{\mathrm{d}}$ are the $\mathrm{CB}$ concentrations in the protozoan $\left(\mathrm{mol} \mathrm{CB} / \mathrm{m}^{3}\right)$ and dissolved phase $\left(\mathrm{mol} \mathrm{CB} / \mathrm{m}^{3}\right)$ respectively; $\mathrm{SA}_{\text {prot }}$ is the surface area of the protist $\left(\mathrm{m}^{2}\right)$; $D_{m}$ is the diffusion coefficient of a CB through the lipid membrane $\left(\mathrm{m}^{2} / \mathrm{s}\right) ; K_{\mathrm{lw}}$ is the lipid-water partition coefficient; and $\Delta \mathrm{z}$ is the thickness of the lipid membrane ( $\mathrm{m}$ ). Values are contained in Table 2-1 below.

One of the key parameters in this calculation is the diffusion coefficient in the lipid membrane. Literature values of $\mathrm{D}_{\mathrm{m}}$ could not be found for any chlorobiphenyls so this parameter was estimated in the following manner. The membrane diffusion coefficient, $\mathrm{D}_{m}$, was estimated for a model compound, $n$-hexanol, chosen because it was the most appropriate compound from the data presented in Stein (1986). This coefficient was then used to estimate $\mathrm{D}_{\mathrm{m}}$ for the $\mathrm{CB}$ congener considered here. The approach and data are all derived from Stein (1986). The diffusion coefficient, $\mathrm{D}_{\mathrm{m}}$, is a function of the basal permeability, $\mathrm{P}(\mathrm{cm} / \mathrm{s})$, the width of the hydrophobic interior of the phospholipid bilayer in the cellular membrane, $\lambda(\AA)$ and the membrane water partition coefficient $(\mathrm{K})$ according to:

$$
\text { (3) } D_{m}=\frac{P \lambda}{K}
$$

$\mathrm{K}$ is actually the hexadecane-water partition coefficient, argued by Stein (1986) to be the best proxy for the membrane water partition coefficient. For $n$-hexanol, these values are $\mathrm{P}=3.7 \times 10^{-3} \mathrm{~cm} / \mathrm{s}$ (Brahm, 1983), $\lambda=40 \AA$ (Stein, 1986), and $\mathrm{K}=1.3$ (Aveyard and Mitchell, 1969). The resultant $D_{m}$ is $2.67 \times 10^{-13} \mathrm{~m}^{2} / \mathrm{s}$. 
Schwarzenbach et al. (1993) present an approximate relationship between the diffusion coefficients and molecular weights of a known and unknown compound:

$$
\text { (4) } \frac{D_{m}(\text { unknown })}{D_{m}(\text { known })}=\left(\frac{M W(\text { known })}{M W(\text { unknown })}\right)^{0.5}
$$

The exponent, 0.5 , on the molecular weight ratio is very rough and will change in different media, especially if viscosity of the media or diffusant conformation is important (Schwarzenbach et al., 1993). Nonetheless, this relationship can be used to give a rough estimate of $D_{m}$ for $C B$ congener IUPAC \#77. The value calculated using the Schwarzenbach et al. (1993) relationship is $1.58 \times 10^{-13} \mathrm{~m}^{2} / \mathrm{s}$. Stein (1986) showed a greater molecular weight dependence of $D_{m}$ in a membrane than in water (steeper slope in $\log \mathrm{D}_{\mathrm{m}}$ versus MW plot). The data shown in Stein (1986) can be approximately described by: $\log \mathrm{D}_{\mathrm{m}}=(-0.033)^{*} \mathrm{MW}-4.8$ where $\mathrm{MW}$ is the molecular weight. This relationship gives $D_{\mathrm{m}}$ for $\mathrm{CB} \# 77$ to be $10^{-15} \mathrm{~cm}^{2} / \mathrm{s}$ or $10^{-19} \mathrm{~m}^{2} / \mathrm{s}$. Since I had two estimates for $D_{\mathrm{m}}\left(10^{-13}\right.$ and $\left.10^{-19} \mathrm{~m}^{2} / \mathrm{s}\right)$, I used $10^{-16} \mathrm{~m}^{2} / \mathrm{s}$ as a compromise value.

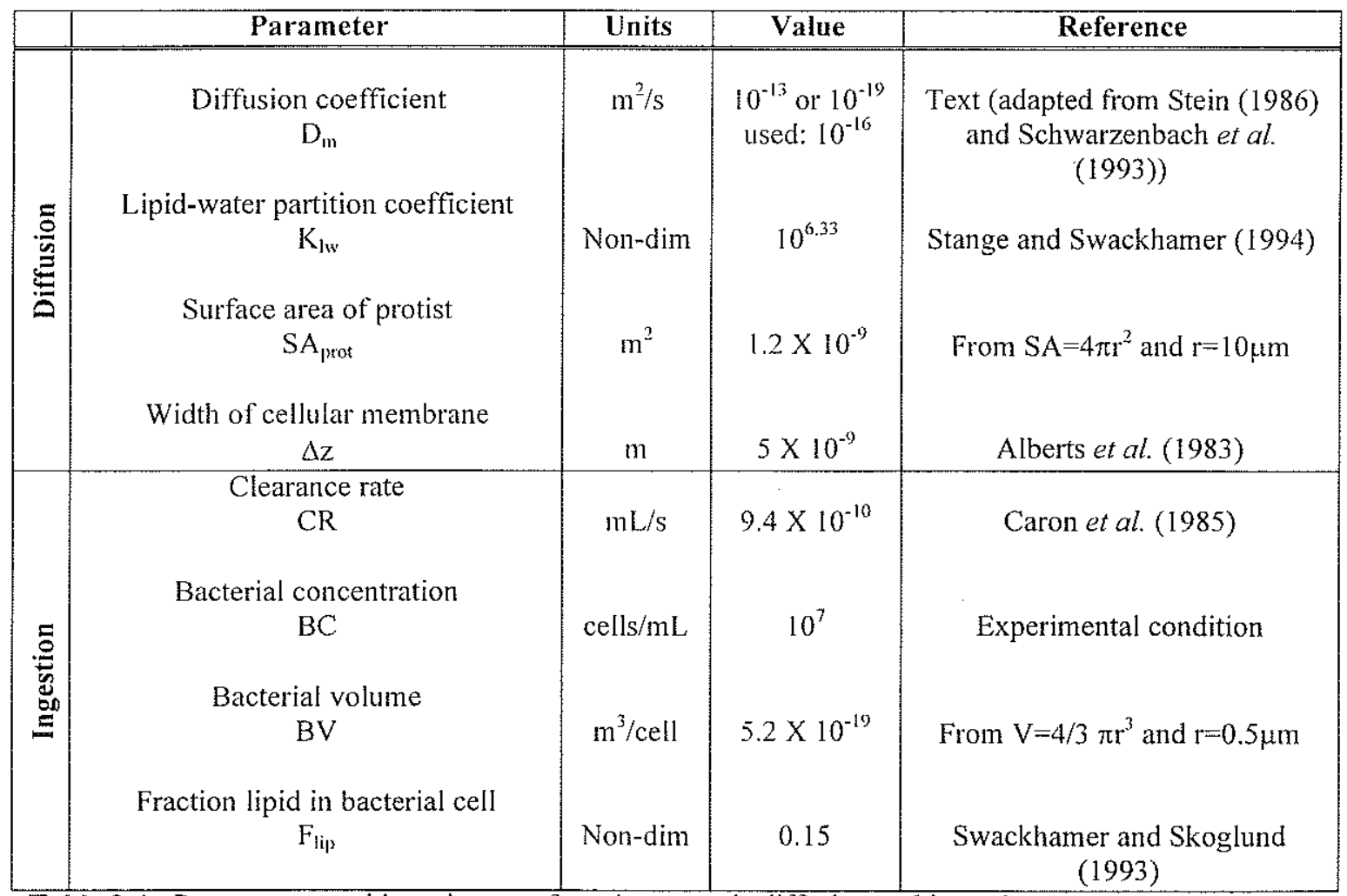

Table 2-1. Parameters used in estimates of uptake rates via diffusion and ingestion. 
This calculation also does not include any membrane effects due to incorporation of PCBs within the membrane. Work by Sikkeman et al. (1994) has shown that even small concentrations $(0.01 \mu \mathrm{mol} / \mathrm{mg}$ phospholipid $)$ of hydrophobic compounds in the membrane caused an expansion of the membrane. This swelling resulted in increased membrane fluidity and permeability. Hydrophobic compounds accumulated in the hydrophobic interior of the phospholipid bilayer according to a lipid-water partition coefficient which was in turn related (log-linear) to the octanol-water partition coefficient. Their study did not find increased uptake due to membrane changes; instead contaminant concentrations in the membrane adhered to physico-chemical expectations.

Uptake via ingestion is equal to prey ingestion rate multiplied by the prey $\mathrm{CB}$ concentration as expressed by the following equation:

$$
\text { (5) }\left(\frac{d[C B]_{p r o t}}{d t}\right)_{i n g}=C R^{*} B C * B V *[C B]_{\text {loact }}
$$

where $[\mathrm{CB}]_{\text {prot }}$ and $[\mathrm{CB}]_{\text {bact }}$ are the $\mathrm{CB}$ concentrations in the protozoa and the bacteria $\left(\mathrm{mol} \mathrm{CB} / \mathrm{m}^{3}\right)$, respectively; $\mathrm{CR}$ is the protozoan clearance rate $(\mathrm{mL} / \mathrm{s}) ; \mathrm{BC}$ is the bacterial concentration (cells $/ \mathrm{mL}$ ); and $\mathrm{BV}$ is the bacterial cell volume $\left(\mathrm{m}^{3} /\right.$ cell). $[\mathrm{CB}]_{\text {bact }}$ is the product of the bacterial lipid fraction $\left(F_{l i p}\right)$, the lipid-water partition coefficient $\left(\mathrm{K}_{\mathrm{lw}}\right)$, and the dissolved $\mathrm{CB}$ concentration $\left([\mathrm{CB}]_{\mathrm{d}}\right)$. The ratio of diffusive uptake (Diff) to ingested uptake (Ing) shows the relative speed of CB influx via the two methods and is represented by the following equation:

$$
\text { (6) } \frac{\text { Diff }}{I n g}=\frac{D_{m}^{*} K_{l w}^{*} S A_{p r o t} *[C B]_{d}}{\Delta z^{*} C R^{*} B C^{*} B V^{*} F_{l i p} * K_{l w} *[C B]_{d}}=3.3 X 10^{4}
$$

Using the parameters described above, this estimate for the diffusion of a tetrachlorobiphenyl predicts that diffusion through the membrane delivers PCBs faster to the cell than ingestion of contaminated prey by a factor of $10^{4}$. This is an end-member estimate because a reasonably low value for diffusive uptake (middle of range of $D_{m}$ ) and the upper limit for ingested uptake (100\% assimilation at maximum clearance rate) are used. 
As mentioned above, diffusion through the unstirred water boundary layer is not addressed in this calculation because of the complication of the cilia on the surface of the protozoan cell. An estimate of the importance of a diffusive boundary layer can be calculated (presented in full in Appendix A). This "back-of-the-envelope" calculation suggested that a boundary layer of $1100 \mu \mathrm{m}$ or larger would impede the rate of diffusive uptake such that ingestion could become the dominant uptake pathway (if diffusion rate = $3.3 \times 10^{4} *$ ingestion rate). Since the organisms in this study are approximately $10-15 \mu \mathrm{m}$ in diameter, a diffusive boundary layer of $1100 \mu \mathrm{m}$ is most likely unrealistically large. In addition, the organisms are swimming through the water, essentially lowering the boundary layer thickness even more. It is unlikely, then, that the presence of a diffusive boundary layer surrounding the ciliate will inhibit diffusive uptake of PCBs. In addition, this equation does not include ingestion of PCBs associated with DOC. See Chapter 3 for a complete discussion.

In this chapter, I present the results of a study designed to verify experimentally the predicted significance of diffusion and ingestion as $\mathrm{CB}$ uptake pathways in protozoans. I compared CB uptake in prey-limited and prey-replete laboratory cultures of protozoa. Bioconcentration factors were calculated and compared in the two experimental treatments. These results showed that diffusion was the dominant uptake pathway and that protozoa rapidly equilibrated with dissolved CB concentrations in the surrounding aqueous medium. To quantify the diffusion process in this system, protozoan uptake and loss rate constants were measured using a radio-labeled congener. These rate constants were compared with an estimated bacterial loss constant using a four-box numerical model. The model results were consistent with the timing of equilibration observed in the original bioaccumulation experiment.

\subsection{Methods}

\subsubsection{Growth of organisms}

Vineyard Sound seawater (VSW) was used in all growth media. The seawater was collected using a Masterflex pump in the spring of 1998 during an incoming tide, Woods 
Hole, MA. Seawater was stored in polycarbonate carboys in the dark at room temperature. Before media preparation, the seawater was filtered once through a $1.2 \mu \mathrm{m}$ in-line Versapor filter (Gelman) and once through a $0.2 \mu \mathrm{m}$ in-line nylon filter (Whatman). The $0.2 \mu \mathrm{m}$-filtered water was autoclaved for at least $30 \mathrm{~min}$ and then stored at room temperature until used for growth media. All glassware used for culturing was washed with Citranox ${ }^{\circledR}$ detergent (Fisher Scientific), soaked in $10 \%$ ethanol $/ \mathrm{HCl}$ in Milli-Q water overnight, rinsed with Milli-Q water and sterilized.

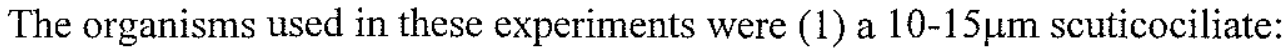
Uronema sp., clone BBCil and (2) a $0.5 \mu \mathrm{m}$ marine bacterium: Halomonas halodurans, both from the collection of D. Caron, University of Southern California (USC), CA. Both organisms were chosen because of their relative hardiness during experimental manipulations. The bacterium species is ubiquitous in the marine environment and can be assumed to be representative of marine heterotrophic bacteria (Barbeau, 1998).

A variation on the protocol of Lim et al. (1993) was used to grow the ciliates to high concentrations $\left(10^{5}-10^{6}\right.$ cells $/ \mathrm{mL}$ ). First, bacteria (Halomonas halodurans) were grown overnight on $0.04 \%$ yeast extract in sterile, $0.2 \mu \mathrm{m}$-filtered VSW. The bacteria were then centrifuged at $11,180 \mathrm{Xg}$ (centrifuge: Biofuge $22 \mathrm{R}$, Heraeus) for $20 \mathrm{~min}$ at $15^{\circ} \mathrm{C}$ and resuspended in sterile VSW. The bacterial pellet was resuspended in one-half the total volume. This was repeated twice (three times total) to ensure complete removal of excess yeast extract. An aliquot of the bacterial concentrate was added to a sterile $2.5 \mathrm{~L}$ culture flask and diluted with sterile VSW to a final volume of $1 \mathrm{~L}$ and a bacterial concentration of $10^{8}-10^{9}$ cells $/ \mathrm{mL}$. Approximately 500-1000 Uronema cells were added to the bacteria. The cultures were shaken on a table rotary shaker at 30-40rpm to ensure an oxygenated medium. Bacterial and Uronema cell concentrations were monitored every 6 hours for 36-48 hours. To determine cell concentrations, culture aliquots were fixed in $1 \%$ glutaraldehyde ( $/ / \mathrm{v}$ VSW) and counted by phase contrast on a Zeiss standard $16 \mathrm{WL}$ microscope. Bacterial cells were counted at $400 \mathrm{X}$ using a Neubauer counter and ciliates were counted at 200X using a Palmer-Maloney cell. 
A two-step centrifugation protocol was developed to selectively remove bacterial prey from protozoan cultures. Aliquots $(40 \mathrm{~mL})$ of protozoan culture were centrifuged in polycarbonate tubes at $6800 \mathrm{rpm}(5169 \mathrm{Xg})$ for $17 \mathrm{~min}$. The top two-thirds of each centrifuge tube were removed as quickly as possible by vacuum aspiration. Fresh VSW was added to each tube to return the volume to the original level ( $40 \mathrm{~mL}$ in this case). The tubes were then mixed to resuspend the bottom pellet and centrifuged again at $6000 \mathrm{rpm}(4024 \mathrm{Xg}$ ) for $12 \mathrm{~min}$ to sediment bacterial aggregates. The tubes were then left undisturbed to allow the protozoans to swim away from the bacterial pellet at the bottom. After 15-20min, the supernatant was removed with a pipet while avoiding any dislodged bacterial aggregates. This supernatant was considered the "protozoan concentrate". The recovery of protozoans from this separation protocol varied greatly depending on the condition of protists prior to centrifugation and the composition of the culture medium. Protozoan cell recoveries varied from $60 \%$ to $10 \%$ (range of all trials performed). Bacterial cell concentrations were reduced by $50 \%$ to $90 \%$ (range of all trials performed high protozoan recoveries not necessarily coincidental with high bacterial recoveries). This reduction was enough to lower the bacterial cell concentrations in the concentrate to $10^{5}$ cells $/ \mathrm{mL}$ or lower, below the grazing threshold of $10^{6}$ cells $/ \mathrm{mL}$. The grazing threshold is an approximate bacterial concentration below which protozoa cannot acquire prey effectively.

\subsubsection{Experimental protocol.}

The PCBs used in this experiment were purchased from AccuStandard (Cat\# CCCSEC-R, Lot \#124-269, New Haven, CT) as a mixture of 21 congeners (approximately $100 \mu \mathrm{g} / \mathrm{mL}$ per congener in acetone) spanning the range of hydrophobicities (Table 2-2). The solution was transferred into a $4 \mathrm{~mL}$ vial and diluted with acetone to approximately $25 \mu \mathrm{g} / \mathrm{mL}$ (per congener). A "working" solution (approximately $200 \mathrm{ng}$ congener $/ \mathrm{mL}$ ) was made by diluting the stock solution with acetone. All experimental cultures contained approximately $0.4 \mathrm{ng} / \mathrm{mL}$ (total) of each CB congener. 
Four 2.5L Fernbach flasks were used in this experiment -2 designated as grazing flasks and 2 designated as non-grazing flasks. The $\mathrm{CB}$ spike $(13 \mu \mathrm{L})$ was added to $450 \mathrm{~mL}$ sterile VSW in the non-grazing flasks. The flasks were shaken on a rotary table shaker for an hour prior to an experiment. For the grazing flasks, the CB spike $(39 \mu \mathrm{L})$

\begin{tabular}{|c|c|c|}
\hline $\begin{array}{l}\text { Congener \# } \\
\text { (IUPAC) }\end{array}$ & Structure & $\log K_{o w}$ \\
\hline 8 & 2,4'-dichlorobiphenyl & 5.07 \\
\hline 18 & $2,2,5-$ & 5.24 \\
\hline 28 & $2,4,4^{\prime}-$ & 5.67 \\
\hline 44 & $2,2^{\prime}, 3,3^{\prime}-$ & 5.75 \\
\hline 52 & $2,2^{\prime}, 5,5^{\prime}-$ & 5.84 \\
\hline 66 & $2,3^{\prime}, 4,4^{\prime}-$ & 6.2 \\
\hline 77 & $3,3^{\prime}, 4,4^{\prime}-$ & 6.36 \\
\hline 101 & $2,2^{\prime}, 4,5,5^{\prime}-$ & 6.38 \\
\hline 105 & $2,3,3^{\prime}, 4,4^{\prime}-$ & 6.65 \\
\hline 118 & $2,3^{\prime}, 4,4^{\prime}, 5-$ & 6.74 \\
\hline 126 & $3,3^{\prime}, 4,4^{\prime}, 5-$ & 6.89 \\
\hline 128 & $2,2^{\prime}, 3,3^{\prime}, 4,4^{\prime}-$ & 6.74 \\
\hline 138 & $2,2^{\prime}, 3,4,4^{\prime}, 5^{\prime}-$ & 6.83 \\
\hline 153 & $2,2^{\prime}, 4,4^{\prime}, 5,5^{\prime}-$ & 6.92 \\
\hline 170 & $2,2^{\prime}, 3,3^{\prime}, 4,4^{\prime}, 5-$ & 7.27 \\
\hline 180 & $2,2^{\prime}, 3,4,4^{\prime}, 5,5^{\prime}-$ & 7.36 \\
\hline 187 & $2,2^{\prime}, 3,4^{\prime}, 5,5^{\prime}, 6-$ & 7.17 \\
\hline 195 & $2,2^{\prime}, 3,3^{\prime}, 4,4^{\prime}, 5,6-$ & 7.56 \\
\hline 199 & $2,2^{\prime}, 3,3^{\prime}, 4,5,6,6^{\prime}-$ & 7.2 \\
\hline 206 & $2,2^{\prime}, 3,3^{\prime}, 4,4^{\prime}, 5,5^{\prime}, 6-$ & 8.09 \\
\hline 209 & decachlorobiphenyl & 8.18 \\
\hline
\end{tabular}

Table 2-2. CB congeners used in experiments - IUPAC \#, structure and $\log \mathrm{K}_{\mathrm{ow}}$.

*-from Hawker and Connell (1988).

was added to a bacterial slurry and shaken on a table rotary shaker for an hour. Bacterial concentrate $(50 \mathrm{~mL})$ was added to $400 \mathrm{~mL}$ VSW in each of the two grazing flasks.

Protozoan concentrate $(550 \mathrm{~mL})$ was added to all four flasks, yielding a total volume of $1 \mathrm{~L}$ in each flask. The addition of protists was considered the start of the experiment. The protozoan concentration in each of the four flasks was approximately $10^{3}$ cells $/ \mathrm{mL}$. The bacterial concentrations in the grazing and non-grazing flasks were $10^{7}$ and $10^{4}$ cells $/ \mathrm{mL}$, respectively, at $\mathrm{t}=2 \mathrm{~h}$. The bacterial concentrations increased over the course of the experiment in all flasks (Table 2-3). 
Samples were taken every $6 \mathrm{~min}$ (on average) for the first hour and then every two hours until the end of the experiment at $\mathrm{t}=6 \mathrm{~h}$. At each timepoint, $40 \mathrm{~mL}$ aliquots were removed from the cultures and filtered through 5.0 $\mu \mathrm{m}$ silver $(\mathrm{Ag})$ filters (Osmonics, Livermore, CA) using positive pressure reverse-flow filtration. Sample aliquots were

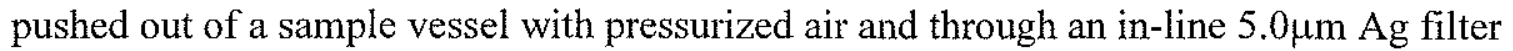
housed in a $47 \mathrm{~mm}$ stainless steel in-line filter holder (Gelman). The sample vessel was a combusted glass Erlenmeyer flask within a Teflon filtration jar. All tubing in the system was stainless steel. The filtration system was cleaned with Milli-Q water and acetone between samples. Size fractionation through $5.0 \mu \mathrm{m}$ silver filters was used to separate bacteria from protozoa. Silver membranes were chosen for this purpose because of low retention of dissolved $\mathrm{PCBs}$ and clean separation of the two organisms.

Filters were covered with 1:1 hexane:acetone in a $40 \mathrm{~mL}$ combusted glass screwcap vial and stored in the refrigerator until analysis. Filtrates were stored in $40 \mathrm{~mL}$ combusted glass vials. At later time points ( $2 \mathrm{~h}$ and onward), $9 \mathrm{~mL}$ aliquots were removed and preserved with $1 \%$ glutaraldehyde for measuring population numbers. In addition, $40 \mathrm{~mL}$ aliquots were removed and stored in combusted glass screw-cap vials to measure total PCBs in each flask. At the last time point (6h), aliquots were filtered through $0.2 \mu \mathrm{m}$ $\mathrm{Ag}$ filters $-40 \mathrm{~mL}$ of each non-grazing, or "diffusion", flask and $40 \mathrm{~mL}$ of a 1:2 dilution of each grazing, or "ingestion", flask. All size fractionations $(5.0 \mu \mathrm{m}$ and $0.2 \mu \mathrm{m})$ were repeated at the $6 \mathrm{~h}$ time point for organic carbon analyses. Filters for organic carbon analyses were folded into quarters, wrapped in combusted $\mathrm{Al}$ foil and stored in $\mathrm{a}-4^{\circ} \mathrm{C}$ freezer until analysis.

\subsubsection{CB Analyses.}

Congeners 14 (3,5-dichlorobiphenyl) and 198 (2,2',3,3',4,5,5',6octachlorobiphenyl) were used as surrogate recovery standards (SRS) in all samples. Congener 103 (2,2',4,5',6-pentachlorobiphenyl) was used as the GC external quantitation standard (QS). The individual congeners were purchased from AccuStandard (Lot \#024-

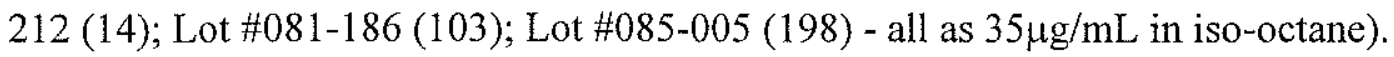


Working solutions were made by diluting the original solutions with iso-octane to a final concentration of approximately $30 \mathrm{pg} / \mu \mathrm{L}$. Prior to use in these analyses, anhydrous $\mathrm{Na}_{2} \mathrm{SO}_{4}$ (Fisher Scientific) was combusted for at least 4 hours at $450^{\circ} \mathrm{C}$ and stored in a desiccator. All solvents (hexane and acetone) were Ultra Resi-Analyzed grade (JT Baker, Phillipsburg, NJ).

At least $12 \mathrm{~h}$ prior to analysis, $150 \mu \mathrm{L}$ of each surrogate recovery standard were added to each sample. Filters were extracted three times by sonic probe extraction (VibraCell, Sonics \& Materials, Inc., Danbury, CT - conditions: pulse for $15 \mathrm{~min}$ at $60 \%$ duty cycle with output 5.0). After each extraction, the solvent was decanted into a roundbottom flask and fresh 1:1 hexane:acetone was added to the filter. All extracts were combined in a round bottom flask. Aqueous samples were decanted into a $125 \mathrm{~mL}$ separatory funnel and acidified with hexane-extracted $1 \mathrm{~N} \mathrm{HCl}$ (4-5 drops) to $\mathrm{pH} 2-3$ to prevent emulsions. The filtrates were extracted five times with hexane. All extracts (and surface emulsions, if any) were combined in a round-bottom flask and dried with anhydrous $\mathrm{Na}_{2} \mathrm{SO}_{4}$. Each extract was solvent-exchanged into hexane and reduced in volume to $1-2 \mathrm{~mL}$ via rotary evaporation.

The presence of emulsions in aqueous extractions was correlated with high bacterial concentrations and resulted in a concomitant loss of PCBs. Due to higher prey abundances, ingestion flask samples were affected to a larger extent by these losses and had lower recoveries (for example, congener \#195 showed losses of up to 50\%). All emulsions were combined with the hexane phase of an extraction. It is possible that PCBs were caught in the anhydrous $\mathrm{Na}_{2} \mathrm{SO}_{4}$ used for dehydration.

The extracts were then cleaned with concentrated $\mathrm{H}_{2} \mathrm{SO}_{4}$ after the method of Bergen et al. (1993). Each extract was added to half its volume of concentrated $\mathrm{H}_{2} \mathrm{SO}_{4}$ in a combusted $15 \mathrm{~mL}$ glass tube. The sealed tube was vortexed for $1 \mathrm{~min}$ and then allowed to sit at least $45 \mathrm{~min}$. The hexane phase was removed and the acid phase was re-extracted twice more with hexane. All hexane phases were combined in a $4 \mathrm{~mL}$ combusted glass vial. Volumes of clean extracts were reduced to approximately $150 \mu \mathrm{L}$ with ultra highpurity $\mathrm{N}_{2}$ after the addition of $150 \mu \mathrm{L}$ of GC quantitation standard. 
Final extracts were transferred to a combusted GC vial with $200 \mu \mathrm{L}$ insert and analyzed on a gas chromatograph (HP 5890 Series II) with an electron capture detector (HP Model \#G1223A) and a 60m DB-5 capillary column (0.25 $\mu \mathrm{m}$ i.d., JT Baker) installed. Analysis conditions consisted of the following temperature program: $60^{\circ} \mathrm{C}$ for $2 \mathrm{~min}$, ramp at $6^{\circ} \mathrm{C} / \mathrm{min}$ to $170^{\circ} \mathrm{C}$, ramp at $1^{\circ} \mathrm{C} / \mathrm{min}$ to $240^{\circ} \mathrm{C}$, hold for $10 \mathrm{~min}$, ramp at $3^{\circ} \mathrm{C} / \mathrm{min}$ to $298^{\circ} \mathrm{C}$ and hold for $5 \mathrm{~min}$ - with $\mathrm{He}$ as a carrier gas flowing at $1.2 \mathrm{~mL} / \mathrm{min}$. Standards were run every six samples to correct for any changes in column conditions. Chromatograms were integrated with HP ChemStation software using a 5-point external standard curve. The volume of the extract was determined from the GC quantitation standard. The calculated volume was then used to determine the amount of surrogate recovery standards expected in the extract. The raw $\mathrm{CB}$ spike data was corrected for recovery by using each of the recoveries of the surrogate recovery standards. The two quantities were then averaged. SRS recoveries averaged $91.6 \% \pm 20.2 \%$ for $\# 14$ and $90.7 \% \pm 17.6 \%$ for $\# 198$ (range: $\# 14-52.2 \%-155.1 \%$; \#198 - 52.3\%-149.3\%; $n=99$ ). GC detection limits were in the pg range for the congeners studied.

\subsubsection{Organic carbon analyses}

$\mathrm{Ag}$ filters were removed from the freezer and allowed to thaw and dry overnight in a $60^{\circ} \mathrm{C}$ oven. The filters were then weighed and cut into quarters. Each quarter to be analyzed was weighed, folded, and wrapped in a Sn boat (Microanalysis, Manchester, MA). The quarters were then combusted and analyzed on a Fisons Instruments EA 1108 Elemental Analyzer. Three of the four quarters were analyzed and averaged to take into account any heterogeneity on the filter surface.

In the radioactive experiment described below (section 2.3.6.), DOC and total organic carbon (TOC) samples were acidified with $50 \%(\mathrm{v} / \mathrm{v}) \mathrm{H}_{3} \mathrm{PO}_{4}(200 \mu \mathrm{L}$ per $40 \mathrm{~mL}$ sample). DOC concentrations were measured by high-temperature combustion (Peltzer and Brewer, 1993) at UMass-Boston. DOC concentrations were significantly higher than measured TOC concentrations and so contamination was suspected. TOC concentrations were used instead of DOC concentrations for the radioactive experiment to circumvent 
the contamination problem but it should be noted that these values represent an upper limit of actual DOC concentrations.

\subsubsection{Population numbers.}

Acridine orange (AO) was used to stain both the bacteria and protists for enumeration after the method outlined in Lim et al. (1996). Aliquots of glutaraldehydepreserved samples were drawn down onto black polycarbonate filters $(25 \mathrm{~mm}, 0.2 \mu \mathrm{m}$ pore size). The polycarbonate filters were placed on top of glass fiber filters (GF/F, $0.7 \mu \mathrm{m}$ nominal pore size) in glass $25 \mathrm{~mm}$ manifolds (Millipore) to ensure homogenous distribution of cells on the filter surfaces. Each aliquot was stained with acridine orange $(100 \mu \mathrm{L} 0.05 \% \mathrm{AO}(\mathrm{w} / \mathrm{w}$ Milli-Q water) for every $1 \mathrm{~mL}$ of preserved sample). The samples were filtered with a low vacuum $(<10 \mathrm{psi})$. After a rinse with sterile Milli-Q water, the filters were quickly transferred to a moist microscope slide. A drop of Type A immersion oil was placed on the surface of the filter and then covered with a $25 \mathrm{~mm} \mathrm{X}$ $25 \mathrm{~mm}$ cover slip. Slides were sealed with clear nail polish and stored in $\mathrm{a}-4^{\circ} \mathrm{C}$ freezer. Bacterial and protozoan cells were enumerated via epifluorescence microscopy using the following filter set: a BP450-490 exciter filter, an FT510 chromatic beam splitter, and an LP520 barrier filter. All slides were made within two weeks of initial glutaraldehyde preservation.

\subsubsection{Radioactive experiments.}

Short (15min) radioactive experiments were conducted with ${ }^{14} \mathrm{C}$-labeled $3,3^{\prime}, 4,4^{\prime}$ tetrachlorobiphenyl (IUPAC \#77), or ${ }^{14} \mathrm{C}-\mathrm{TCB}$, (specific activity: $52.1 \mu \mathrm{Ci} / \mu \mathrm{mol}-$ courtesy of J. Stegeman, WHOI, MA) to better determine the protozoan uptake rate constant. This experiment was performed using protozoan cultures with low bacteria concentrations and was not repeated with high bacteria concentrations. Filtrates $(<5.0 \mu \mathrm{m})$ of the same protozoan culture were used to test the retention of dissolved PCBs by $\mathrm{Ag}$ filters. In each experiment, an aliquot $(600 \mathrm{~mL})$ of either culture or filtrate was inoculated with ${ }^{14} \mathrm{C}$-TCB (in an acetone carrier) to a final concentration of $0.25 \mathrm{ng} / \mathrm{mL}$ 
(approximately $100 \mathrm{dpm} / \mathrm{mL}$ ). The concentration of radio-label was low relative to typical radio-fractionation studies. However, higher activities would have required higher CB concentrations in terms of mass and the results would have not been comparable to the earlier studies. No loss of sensitivity in measurements was observed since filters and $5-10 \mathrm{~mL}$ of solution were analyzed on the scintillation counter.

The addition of the congener was considered $t_{0}$. Three replicate samples of culture and two replicates of culture filtrate were tested. In each experiment, $50 \mathrm{~mL}$ aliquots of solution were removed as quickly as possible for the duration of the experiment and vacuum-filtered through $5.0 \mu \mathrm{m} \mathrm{Ag}$ filters. The filters were placed in scintillation vials with $5 \mathrm{~mL}$ ScintiVerseII scintillation cocktail (Fisher Scientific) and counted to $\pm 2 \%$ on a Beckman Scintillation Counter (counts ranged from 700 to $1400 \mathrm{dpm}$ per sample). Filter radioactive counts were normalized to total aliquots removed in the middle of the experiment. Prior to ${ }^{14} \mathrm{C}-\mathrm{TCB}$ inoculation, solution aliquots were also removed for bacterial and protozoan cell enumeration as well as analysis of total and dissolved organic carbon $(<0.2 \mu \mathrm{m})$. Blank samples averaged $55 \pm 7 \mathrm{dpm}$ and were subtracted from all experimental samples.

\subsection{Results}

\subsubsection{Bioaccumulation experiments.}

In the prey-limited, or "diffusion", flask, bacterial cell numbers remained below the protozoan grazing threshold until the end of the experiment. In the prey-replete, or "ingestion", flask, however, the prey concentration remained above the grazing threshold during the entire experiment. The protozoan population in each flask did not change significantly over the time course of the experiment (Table 2-3). Qualitatively, however, the health of the protozoa in the two flasks was different. It was observed microscopically that cells in the diffusion flask were very thin and contained few $(<5)$ food vacuoles, whereas the protozoa in the ingestion flask were robust and full of food vacuoles $(15-20)$. 


\begin{tabular}{|c|c|c|c|c|}
\hline $\begin{array}{c}\text { Flask } \\
\text { And Replicate \# }\end{array}$ & $\begin{array}{l}\text { Bacterial cells } \\
\pm 1 \sigma(\text { cells } / \mathrm{mL})\end{array}$ & $\begin{array}{l}\text { Protozoan cells } \\
\pm 1 \sigma(\text { cells } / \mathrm{mL})\end{array}$ & $\begin{array}{c}\text { Organic carbon } \\
\text { in } 5.0 \mu \mathrm{m} \text { fraction } \\
(\mathrm{fg} / \mathrm{mL})\end{array}$ & $\begin{array}{l}\text { Organic carbon } \\
\text { per protozoan } \\
\text { cell }+1 \sigma(f g)\end{array}$ \\
\hline $\begin{array}{c}\text { Diffusion Rep 1 } \\
(\mathrm{t}=2)\end{array}$ & $\begin{array}{l}7.63 \times 10^{4} \\
\left(1.08 \times 10^{4}\right)\end{array}$ & $\begin{array}{r}1.94 \times 10^{5} \\
\left(6.15 \times 10^{2}\right)\end{array}$ & & \\
\hline $\begin{array}{c}\text { Diffusion Rep } 2 \\
(\mathrm{t}=2)\end{array}$ & $\begin{array}{c}4.88 \times 10^{3} \\
\left(2.78 \times 10^{4}\right)\end{array}$ & $\begin{array}{c}5.18 \times 10^{3} \\
\left(8.22 \times 10^{2}\right)\end{array}$ & & \\
\hline $\begin{array}{c}\text { Ingestion Rep } 1 \\
(t=2)\end{array}$ & $\begin{array}{r}1.25 \times 10^{\prime} \\
\left(1.13 \times 10^{5}\right)\end{array}$ & $\begin{array}{r}3.05 \times 10^{5} \\
\left(1.03 \times 10^{3}\right)\end{array}$ & & \\
\hline $\begin{array}{c}\text { Ingestion Rep } 2 \\
(t=2)\end{array}$ & $\begin{array}{c}1.45 \times 10^{\prime} \\
\left(2.00 \times 10^{6}\right)\end{array}$ & $\begin{array}{c}2.78 \times 10^{5} \\
\left(6.57 \times 10^{2}\right)\end{array}$ & & \\
\hline $\begin{array}{c}\text { Diffusion Rep } 1 \\
(t=6)\end{array}$ & $\begin{array}{c}1.21 \times 10^{6} \\
\left(1.31 \times 10^{4}\right)\end{array}$ & $\begin{array}{c}1.39 \times 10^{5} \\
\left(3.16 \times 10^{2}\right)\end{array}$ & $\begin{array}{c}4.94 \times 10^{9} \\
\left(2.97 \times 10^{8}\right)\end{array}$ & $\begin{array}{c}3.56 \times 10^{6} \\
\left(8.38 \times 10^{5}\right)\end{array}$ \\
\hline $\begin{array}{c}\text { Diffusion Rep } 2 \\
(\mathrm{t}=6)\end{array}$ & $\begin{array}{c}7.80 \times 10^{6} \\
\left(1.08 \times 10^{5}\right)\end{array}$ & $\begin{array}{c}3.52 \times 10^{5} \\
\left(5.55 \times 10^{2}\right)\end{array}$ & $\begin{array}{c}7.80 \times 10^{9} \\
\left(1.98 \times 10^{8}\right)\end{array}$ & $\begin{array}{c}2.22 \times 10^{6} \\
\left(3.55 \times 10^{5}\right)\end{array}$ \\
\hline $\begin{array}{c}\text { Ingestion Rep } 1 \\
(t=6)\end{array}$ & $\begin{array}{c}2.53 \times 10^{7} \\
\left(2.95 \times 10^{6}\right)\end{array}$ & $\begin{array}{c}4.44 \times 10^{3} \\
\left(1.03 \times 10^{3}\right)\end{array}$ & $\begin{array}{c}6.35 \times 10^{7} \\
\left(2.51 \times 10^{7}\right)\end{array}$ & $\begin{array}{c}1.43 \times 10^{5} \\
\left(3.33 \times 10^{5}\right)\end{array}$ \\
\hline $\begin{array}{c}\text { Ingestion Rep } 2 \\
(t=6)\end{array}$ & $\begin{array}{c}2.12 \times 10^{\prime} \\
\left(3.19 \times 10^{5}\right)\end{array}$ & $\begin{array}{c}2.64 \times 10^{3} \\
\left(7.08 \times 10^{2}\right)\end{array}$ & $\begin{array}{c}1.15 \times 10^{10} \\
\left(1.84 \times 10^{8}\right)\end{array}$ & $\begin{array}{c}4.35 \times 10^{6} \\
\left(1.17 \times 10^{6}\right)\end{array}$ \\
\hline
\end{tabular}

Table 2-3. Population and organic carbon data for all experiments Bacterial and protozoan cell concentrations are the average of 16 random fields corrected for volume aliquot filtered. Errors (in brackets) are $\pm I$ standard deviation. Organic carbon data are average of three filter sections with errors of \pm 1 standard deviation. Organic carbon per cell was calculated by dividing organic carbon concentration by protozoan cell concentration. Errors were propagated from errors on protozoan population counts and organic carbon analyses.

Particulate organic carbon concentrations in the two size classes $(>0.2 \mu \mathrm{m}$ (total particulate carbon) and $>5.0 \mu \mathrm{m}$ (protozoan size fraction)) were similar in the prey-limited flasks because protozoa represented the major particulate pool. Conversely, the total particulate organic carbon $(>0.2 \mu \mathrm{m})$ in the prey-replete cultures was approximately twice that in the $>5.0 \mu \mathrm{m}$ fraction, i.e., the protozoa, due to contributions of bacterial biomass in the $0.2-5.0 \mu \mathrm{m}$ size fraction. Bacterial aggregates constituted a small fraction of the $5.0 \mu \mathrm{m}$ size class in either flask. The percentage of organic carbon represented by bacteria on $5.0 \mu \mathrm{m}$ filters ranged from 0 to $14.5 \%$ with an average of $0.24 \pm 0.31 \%$ in the diffusion flasks and $7.1 \pm 6.4 \%$ in the ingestion flasks. These values were calculated with protozoan cell carbon content (average $=2.9 \times 10^{6} \mathrm{fg} / \mathrm{cell}$ ) from this work and bacterial cell carbon content (70 fg/cell) from Caron et al. (1991). The organic carbon per protozoan cell was calculated in each of the experimental bottles by dividing the organic carbon concentration in the $>5.0 \mu \mathrm{m}$ fraction by the number of protozoans in the filtered aliquot (Table 2-3). 
Significant losses of PCBs were observed over the course of the experiment. The dynamics and magnitude of this loss were consistent with volatilization (Figure 2-1). The masses of each congener occurring in the protozoan size class in both flasks increased rapidly and achieved maximal values within twenty minutes of $\mathrm{CB}$ inoculation (representative congeners 18, 128, 195 in Figure 2-2). Total CB recoveries in the ingestion flask were lower than in the diffusion flask, potentially due to lower volatilization (Figure 2-3). To circumvent volatilization and emulsion complications, congener concentrations were normalized to the total extracted at a time point (Figure 24). The maximum percent of each congener within the protozoan size class $(>5.0 \mu \mathrm{m})$ was achieved quickly. The relative amounts of each congener in the protozoan size class followed the trend expected from the hydrophobicity ( $\mathrm{K}_{\mathrm{ow}}$ values) of the congeners, that high $\mathrm{K}_{\mathrm{ow}}$ congeners (high $\mathrm{Cl}$ number) should have higher concentrations in the organic phase than low $\mathrm{K}_{\mathrm{ow}}$ congeners. Data tables are available in Appendix B. There was no time lag associated with the diffusive uptake pathway. Given this data set, it seems probable that the ingestion pathway does not contribute additional PCBs to the protozoan cell above those assimilated through diffusion. The data is consistent with the hypothesis that $\mathrm{CB}$ uptake is driven by diffusion and the steady-state cellular CB concentration is determined by the hydrophobicity of the $\mathrm{CB}$ congener.

\subsubsection{Comparison of $C B$ aqueous concentrations to $C B$ aqueous solubilities.}

The aqueous concentrations of each $\mathrm{CB}$ congener used were compared to their respective aqueous solubilities in order to show that the concentrations used were significantly different from the saturation concentration. The aqueous solubility of each congener was calculated from its $\log \mathrm{K}_{\text {ow }}$ according to the equation from Schwarzenbach et al. (1993):

$$
\text { (7) } \log C_{w}^{s u t}=\frac{\log K_{o w}-0.78}{-0.85}
$$

where $\mathrm{C}_{\mathrm{w}}^{\text {sat }}$ is the concentration in pure water at saturation $(\mathrm{mol} / \mathrm{L})$ of the subcooled liquid compound at standard temperature and pressure. The saturation concentration was 
corrected for the presence of salts in seawater using the following relationship (also from Schwarzenbach et al. (1993):

$$
\text { (8) } \log \left(\frac{C_{w}^{\text {sut }}}{C_{w, \text { salt }}^{\text {sutt }}}\right)=K^{s}[\text { salt }]
$$

where $\mathrm{C}^{\text {sat }}{ }_{\text {wsalt }}$ is the saturation concentration corrected for salt ions $(\mathrm{mol} / \mathrm{L}), \mathrm{K}^{\mathrm{s}}$ is the Setschenow or salting constant ( 0.3 used for PCBs - from Table 5.6 in Schwarzenbach et $a l$. (1993)) and [salt $]_{\mathrm{t}}$ is the total molar salt concentration $(\approx 0.5 \mathrm{M}$ for seawater $) . \mathrm{C}^{\mathrm{sat}}{ }_{\mathrm{w}}$ and $\mathrm{C}^{\text {sat }}{ }_{\mathrm{w} \text {,salt }}$ were calculated for each congener used in this experiment and then compared to the actual aqueous concentration of each congener at the start of each experiment, $\mathrm{C}_{\mathrm{w}}$ (Table 2-4). In the calculation of $\mathrm{C}_{\mathrm{w}}$, it was assumed that all PCBs were truly dissolved

\begin{tabular}{|c|c|c|c|c|}
\hline Congener & $\mathrm{C}^{\text {Silt }}{ }_{\text {w }}$ & $\mathbf{C}^{\text {sit }}$ w, salt & $\mathrm{C}_{\mathrm{w}}$ & $\mathrm{C}_{\mathrm{w}} / \mathrm{C}^{\mathrm{sit}}{ }_{\mathrm{w}, \text { salt }}$ \\
\hline 8 & $9.0 \mathrm{E}-06$ & $6.5 \mathrm{E}-06$ & $2.0 \mathrm{E}-09$ & 0.00032 \\
\hline 18 & $5.7 \mathrm{E}-06$ & $4.0 \mathrm{E}-06$ & $1.7 \mathrm{E}-09$ & 0.00041 \\
\hline 28 & $1.8 \mathrm{E}-06$ & $1.2 \mathrm{E}-06$ & $1.6 \mathrm{E}-09$ & 0.0013 \\
\hline 44 & 1.4E-06 & $1.0 \mathrm{E}-06$ & 1.4E-09 & 0.0014 \\
\hline 52 & 1.1 E-06 & $7.9 \mathrm{E}-07$ & $1.4 \mathrm{E}-09$ & 0.0018 \\
\hline 66 & $4.2 \mathrm{E}-07$ & $3.0 \mathrm{E}-07$ & $1.4 \mathrm{E}-09$ & 0.0047 \\
\hline 77 & $2.7 \mathrm{E}-07$ & $1.9 \mathrm{E}-07$ & $1.4 \mathrm{E}-09$ & 0.0073 \\
\hline 101 & $2.6 \mathrm{E}-07$ & $1.8 \mathrm{E}-07$ & $1.2 \mathrm{E}-09$ & 0.0066 \\
\hline 105 & $1.2 \mathrm{E}-07$ & $8.8 \mathrm{E}-08$ & $1.2 \mathrm{E}-09$ & 0.014 \\
\hline 118 & $9.7 \mathrm{E}-08$ & $6.9 \mathrm{E}-08$ & $1.2 \mathrm{E}-09$ & 0.018 \\
\hline 126 & $6.5 \mathrm{E}-08$ & $4.6 \mathrm{E}-08$ & $1.2 \mathrm{E}-09$ & 0.026 \\
\hline 128 & $9.7 \mathrm{E}-08$ & $6.9 \mathrm{E}-08$ & $1.1 \mathrm{E}-09$ & 0.015 \\
\hline 138 & $7.6 \mathrm{E}-08$ & $5.4 \mathrm{E}-08$ & 1. $1 \mathrm{E}-09$ & 0.02 \\
\hline 153 & $6.0 \mathrm{E}-08$ & $4.2 \mathrm{E}-08$ & 1.1E-09 & 0.025 \\
\hline 170 & $2.3 \mathrm{E}-08$ & $1.6 \mathrm{E}-08$ & $9.5 \mathrm{E}-10$ & 0.058 \\
\hline 180 & $1.8 \mathrm{E}-08$ & $1.3 \mathrm{E}-08$ & $9.6 \mathrm{E}-10$ & 0.074 \\
\hline 187 & $3.0 \mathrm{E}-08$ & $2.1 \mathrm{E}-08$ & $9.5 \mathrm{E}-10$ & 0.044 \\
\hline 195 & $1.0 \mathrm{E}-08$ & $7.5 \mathrm{E}-09$ & $8.6 \mathrm{E}-10$ & 0.11 \\
\hline 199 & $2.8 \mathrm{E}-08$ & $2.0 \mathrm{E}-08$ & $8.7 \mathrm{E}-10$ & 0.044 \\
\hline 206 & $2.5 \mathrm{E}-09$ & $1.8 \mathrm{E}-09$ & $7.8 \mathrm{E}-10$ & 0.44 \\
\hline 209 & $2.0 \mathrm{E}-09$ & $1.4 \mathrm{E}-09$ & $7.2 \mathrm{E}-10$ & 0.52 \\
\hline
\end{tabular}

Table 2-4. Aqueous solubilities, concentrations and comparisons for all CB congeners used. All concentrations presented are in units of $\mathrm{mol} / \mathrm{L} . \mathrm{C}^{\text {sat }}$ iv is the saturation concentration in water at standard conditions and $\mathrm{C}^{\mathrm{Sat}}$ w,satt is $\mathrm{C}^{\text {sat }}{ }_{\mathrm{w}}$ corrected for the presence of salt ions in seawater. $\mathrm{C}_{\mathrm{w}}$ is the actual $\mathrm{CB}$ concentration in the experiment described in this chapter. The ratio of $\mathrm{C}_{\mathrm{w}}$ to $\mathrm{C}_{\mathrm{w}, \text { sait }}^{\text {sat }}$ gives an indication of the distance from saturation in the experiment. 
and no complexation with organic carbon (dissolved or otherwise) occurred. For most congeners, $\mathrm{C}_{\mathrm{w}}$ in the experiment was significantly lower than $\mathrm{C}^{\text {sat }}{ }_{\text {,salt. }}$ Congeners 195 , 206 and 209 had $\mathrm{C}_{\mathrm{w}}$ 's greater than $10 \%$ of $\mathrm{C}_{\mathrm{w}, \text { salt. }}^{\mathrm{sat}}$.

\subsubsection{Bioconcentration factors.}

Bioconcentration factors (BCFs) were calculated for each congener at the last time point in both diffusion and ingestion flasks (because organic carbon and $<0.2 \mu \mathrm{m} \mathrm{CB}$ samples were available only for $\mathrm{t}=6 \mathrm{~h}$ ). BCFs are defined as the $\mathrm{CB}$ concentration in the biological phase divided by the $\mathrm{CB}$ concentration in the surrounding medium. This calculation should be equivalent to the definition of $\mathrm{K}_{\mathrm{oc}}$, the organic carbon to water partition coefficient. In this case, the biological PCBs were normalized to total particulate organic carbon ( $\mathrm{g} \mathrm{CB} / \mathrm{g} \mathrm{OC}$ ) and the aqueous PCBs were assumed to be equivalent to the PCBs measured in the $0.2 \mu \mathrm{m}$ filtrate. The BCFs calculated in this fashion are presented in Figure 2-5 along with the predicted $\mathrm{K}_{\mathrm{oc}}$ values (Schwarzenbach et al., 1993) for each congener. The BCF values for each congener were not statistically different in the two flasks, suggesting that the PCBs have been concentrated in the biological phase of each experimental flask according to organic carbon content - notably despite differences in lipid-rich vacuole concentration. If different mechanisms were occurring in the diffusion and ingestion flasks, I would expect differences in the BCFs between congeners in different flasks.

The predicted $\mathrm{K}_{\mathrm{oc}}$ and measured BCFs diverge above $\log \mathrm{K}_{\mathrm{ow}}=6.5$. This may be due to uncertainties in the denominator of the $\mathrm{BCF}$ calculation, the dissolved $\mathrm{CB}$ concentration. The $\mathrm{CB}$ concentrations in the $0.2 \mu \mathrm{m}$ filtrate at the final time point were assumed to be equivalent to the truly dissolved PCBs. This assumption is likely not valid but there were no reliable estimates for PCBs associated with colloids or DOC ([CB] $]_{\text {DOC }}$ ) in this experiment. In both flasks, both the measured $\log \mathrm{BCFs}$ and predicted $\log \mathrm{K}_{\mathrm{oc}}$ 's increased with $\log \mathrm{K}_{\mathrm{ow}}$ up to 6.5. Predicted $\log \mathrm{K}_{\mathrm{oc}}$ is independent of $\log \mathrm{K}_{\mathrm{ow}}$ above $\log$ $\mathrm{K}_{\mathrm{ow}}=7.5$. The independence of initial BCF on $\mathrm{K}_{\mathrm{ow}}$ above $\log \mathrm{K}_{\mathrm{ow}}=6.5$ has been observed by other investigators (Skoglund and Swackhamer, 1994). They assumed that 
this plateau in particulate $\mathrm{CB}$ concentrations indicated the presence of a short-term surface adsorption constant that was independent of congener hydrophobicity (Skoglund et al., 1996). They hypothesized subsequent slow secondary uptake into internal cellular pools.

The uniformity of the BCFs in the two experimental flasks suggests that the PCBs have been assimilated into all organic carbon-containing cellular compartments. If so, another explanation is necessary for the plateau effect described above. $[\mathrm{CB}]_{\mathrm{DOC}}$ is larger for the more chlorinated congeners and so the separation between predicted and measured particulate fractions should be related to the PCBs associated with colloidal or dissolved organic material. I estimated the DOC concentration needed to generate the observed difference between $\log \mathrm{K}_{\mathrm{oc}}$ and $\log \mathrm{BCF}$ for IUPAC \#180 $\left(\log \mathrm{K}_{\mathrm{oc}}=6.2\right)$ to be $7.3 \mathrm{mg} / \mathrm{L}$. This value is within the range of DOC concentrations observed in these cultures (2$15 \mathrm{mg} / \mathrm{L}-$ see Chapter 4$)$. Regrettably, this hypothesis cannot be confirmed without DOC concentrations (measured only in the radioactive experiments described in the next section). However, the possibility that these cultures contain material that binds PCBs prompts further questions regarding the role of protozoan grazing in $\mathrm{CB}$ speciation in natural settings. It is also interesting that the effect of this material appears to be congener-specific, causing larger deviations from predicted $\mathrm{K}_{\mathrm{oc}}$ 's for the more chlorinated congeners.

\subsubsection{Coplanar vs. non-coplanar congeners.}

The difference between the diffusive and ingested uptake pathways may be a subtler one than bulk PCB cellular content. The diffusive pathway can discriminate against a congener based on size and/or structure whereas the ingested pathway incorporates all congeners uniformly (assuming no discrimination across the vacuole membrane). It has been suggested in the literature that $\mathrm{CB}$ congeners that can achieve a coplanar conformation can enter a membrane more easily than those with chlorine atoms in ortho positions (Kannan et al., 1989). To determine whether there was evidence of this subtle effect in the present data set, the ratio of non-coplanar congeners to coplanar 
yet equally chlorinated (same molecular weight) congeners was calculated in both experimental treatments. The ratios of these congeners were expected to follow $\mathrm{K}_{\mathrm{ow}}$ considerations, i.e., if the non-coplanar congener had a higher $\mathrm{K}_{\mathrm{ow}}$ than the coplanar congener, the ratio of non-coplanar to coplanar congener should be greater than 1 . This ratio should remain constant with time if no discrimination were occurring. The data was inconclusive on this point (Figure 2-6). The ratio of \#126 to \#101 should be constant and the ratio of \#126 to \#153 should be less than 1. Including ratios from replicate bottles as well as previous experiments did nothing to elucidate any trends. The error bars were too large and the time scale was not long enough to discern any real differences between the coplanar and noncoplanar congeners (Figure 2-6).

\subsubsection{Radioactive diffusion experiments.}

The Ag filters adsorbed a small fraction of the ${ }^{14} \mathrm{C}-\mathrm{TCB}$ from the $5.0 \mu \mathrm{m}$ filtrate (average: $15.5 \pm 2.0 \mathrm{dpm} / \mathrm{mL}$ filtered; $n=34$ - roughly $15 \%$ of the total ${ }^{14} \mathrm{C}-\mathrm{TCB}$ added and consistent with previous wall loss studies). The background filter-associated ${ }^{14} \mathrm{C}$-TCBs were subtracted from the $5.0 \mu \mathrm{m}$ filters to determine the amount associated with the protozoa (data shown in Figure 2-7). The data from the short-term radioactive diffusion experiment was assumed to exhibit pseudo-first order uptake of ${ }^{14} \mathrm{C}-\mathrm{TCB}$ by the protozoa ( $>5.0 \mu \mathrm{m}$ size class $)$ - from Figure 2-7. The data from all three trials were combined and analyzed using the average DOC concentrations. The rate equations of this system included uptake and loss rate constants for the protozoan cells, the organic carbon-water partition coefficient for congener \#77, and DOC concentrations.

The Levenberg-Marquardt Method of the non-linear least squares regression technique was used to find the best fit for the data for both sets of analyses. The following system of equations was solved analytically.

$$
\begin{aligned}
\text { (9) } \frac{d[C B]_{A q}}{d t}= & \frac{k_{r e v}}{[P]^{*}[O C]_{p}}[C B]_{p r o t}-k_{f o r}[C B]_{A q}-[D O C]^{*} * K_{O C} * \frac{d[C B]_{A q}}{d t} \\
& \text { (10) }[C B]_{D O C}=K_{O C} *[D O C] *[C B]_{A q} \\
& \text { (11) }[C B]_{Y o t}=[C B]_{A q}+[C B]_{D O C}+[C B]_{p r o t}
\end{aligned}
$$


where: $[\mathrm{CB}]_{\mathrm{Aq}},[\mathrm{CB}]_{\mathrm{DOC}},[\mathrm{CB}]_{\text {prot }}$ are the $\mathrm{CB}$ concentrations in the aqueous, DOC, and protozoan pools respectively $(\mathrm{dpm} / \mathrm{mL}) ; \mathrm{k}_{\text {for }}$ and $\mathrm{k}_{\text {rev }}$ are the uptake and loss rate constants $\left(\mathrm{min}^{-1}\right)$; $[\mathrm{DOC}]$ is the concentration of DOC $(\mathrm{g} \mathrm{OC} / \mathrm{mL})$; $[\mathrm{P}]$ is the protozoan concentration (cells/mL;) [OC $]_{\mathrm{p}}$ is the organic carbon per protozoan cell ( $\left.\mathrm{g} \mathrm{OC} / \mathrm{cell}\right)$ as determined in the previously described bioaccumulation experiment (Table 2-3); and $\mathrm{K}_{\mathrm{OC}}$ is the organic-carbon/water partition coefficient $((\mathrm{dpm} \mathrm{CB} / \mathrm{g} \mathrm{OC}) /(\mathrm{dpm} \mathrm{CB} / \mathrm{g}$ wat $))$ as described by Schwarzenbach et al. (1993). The analytical solution to this system of equations is:

$$
[C B]_{i q, t}=[C B]_{A q, 0} e^{-X t}+\frac{k_{r e v} *[C B]_{T o t}}{k_{r e v} *\left(1-K_{O C} *\left[D O C D+k_{f o r} *[P]^{*} *[O C]_{P}\right.\right.}\left(1-e^{-X t}\right)
$$

where: $X=\frac{k_{r e v}}{[P]^{*}[O C]_{\mu}}+\frac{k_{f o r}}{1+K_{O C:} *[D O C]}$

The fit of this analytical solution to the radioactive data generated values for the two rate

constants, $\mathrm{k}_{\mathrm{for}}=0.38 \pm 0.03 \mathrm{~min}^{-1}$ and $\mathrm{k}_{\mathrm{rev}}=1.1 \pm 0.1 \times 10^{-5}(\mathrm{gOC})^{-1} \mathrm{~min}^{-1}$. The regression coefficient $\left(\mathrm{R}^{2}\right)$ of the fit was 0.93 (Figure 2-8).

\subsubsection{Calculation of bacterial loss rate constant}

The time scale of protozoan uptake of PCBs and the time scale of bacterial loss of PCBs were then compared. This was done to ensure that the protozoan diffusive uptake could be supplied adequately by loss from the bacterial pool. Experimental determination of the bacterial loss rate constant could not be performed because the analytical method chosen (extraction by Tenax resin - see Chapter 3) was not fast enough. In lieu of experimental determination, the bacterial loss rate constant was estimated using the following calculation.

First, equation 2 from the initial calculation in the chapter introduction was rewritten in terms of the protozoan uptake rate constant:

$$
\left(\frac{d[C B]_{p r o t}}{d t}\right)_{d i t f}=\frac{D_{m} K_{t w} S A_{p r o t}[p r o t}{\Delta z}[C B]_{d i s s}=k_{f o r}[C B]_{d i s s}
$$


where: $k_{f o r}=\frac{D_{m} K_{l w} S A_{p r o t}[\text { prot }]}{\Delta z}$

The theoretical protozoan $\mathrm{k}_{\text {for }}$ was calculated to be $1.5 \times 10^{5} \mathrm{~min}^{-1}$. The calculation was repeated for bacterial cells by substituting appropriate values for cell-specific parameters: $\mathrm{SA}_{\text {bact }}=5 \times 10^{-13} \mathrm{~m}^{2}$ and $[$ bact $]=5 \times 10^{13}$ cells $/ \mathrm{m}^{3}$. All other values were equivalent to those in the protozoan calculation. Using this calculation, the bacterial uptake rate constant, $\mathrm{k}_{\text {up,bact }}$ was estimated at $6.4 \times 10^{5} \mathrm{~min}^{-1}$. At equilibrium, the rates of uptake and loss from the bacteria are equivalent and the ratio of the rate constants equal the organism-based partition coefficient, in this case, $\mathrm{K}_{\mathrm{oc}}$. Therefore, $\mathrm{k}_{\mathrm{dep}}$ was $2.9(\mathrm{~g} \mathrm{OC})^{-1}$ $\min ^{-1}$, or $8.3 \times 10^{5} \mathrm{~min}^{-1}$ when the bacterial concentration and organic carbon content are taken into account. From this calculation, I concluded that the protozoan uptake rate constant and bacterial depuration rate constant are of similar magnitude. Even though the absolute magnitude of these rate constants is much greater than what was measured experimentally, the uncertain parameters (the diffusion coefficient, $D_{m}$, the width of the membrane, $\Delta z$, the partition coefficient, $K_{\mathrm{lw}}$ ) would affect each rate constant by the same amount. The relative equivalence of protozoan uptake and bacterial loss rate constants was applied to the experimental values from the previous section. On the basis of the experimentally derived protozoan uptake rate constant, the bacterial depuration rate constant, $\mathrm{k}_{\mathrm{dep}}$, was estimated to be approximately $0.38 \mathrm{~min}^{-1}$.

\subsubsection{Comparison of protozoan uptake rate and bacterial depuration rate.}

A four-box model was written to compare the protozoan uptake and loss rate constants with the estimated bacterial loss rate constant. In addition, I compared the model results to the data from the bioaccumulation experiments. Initial values for the bacterial, DOC, and aqueous phases were assumed to be equal to the equilibrium values predicted by $\mathrm{K}_{\mathrm{oc}}$. The protozoan size class contained no PCBs at $\mathrm{t}_{0}$. The model was run with $0.1 \mathrm{~min}$ time steps for $60 \mathrm{~min}$. The following equations described the fluxes between pools.

$$
B_{n}^{d i f f}=B_{n-1}^{d i f f}-k_{d e p} * B_{n-1}^{d i f f} * \Delta t
$$




$$
\begin{aligned}
& B_{n}^{i n g}=B_{n-1}^{i n g}-k_{d e p} * B_{n-1}^{i n g} * \Delta t-I R * B_{n-1}^{i n g} * \Delta t \\
& W_{n}=W_{n-1}+Y \\
& Y=k_{d e p} * B_{n-1} * \Delta t-k_{f o r} * A q_{n-1} * \Delta t+k_{r e v} *[P] *[O C]_{P} * P_{n-1} * \Delta t \\
& A q_{0}=\left(1+K_{a c} *[D O C] * W_{0}\right. \\
& A q_{n}=A q_{n-1}+\left(\frac{1}{1+[D O C] * K_{O !}}\right) * Y \\
& D_{n}=W_{n}-A q_{n}=D_{n-1}+\left(\frac{[D O C]^{*} K_{O C}}{1+[D O C]^{*} K_{O C}}\right) * Y \\
& P_{n}^{\text {diff }}=P_{n-1}^{\text {diff }}+k_{\text {jor }} * A q_{n-1} * \Delta t-k_{r e v} * P_{n t-1}^{\text {diff }} *[P]^{*}[O C]_{p} * \Delta t \\
& P_{n}^{i m g}=P_{n-1}^{i n g}+k_{f o r} * A q_{n-1} * \Delta t-k_{r e v} * P_{n-1}^{i n g} *[P] *[O C]_{p} * \Delta t+I R * B_{n-1} * \Delta t
\end{aligned}
$$

$B_{n}, W_{n}, A q_{n}, D_{n}$ and $P_{n}$ refer to the mass of $C B$ in the bacterial, water (aqueous and DOC combined), aqueous, DOC, and protozoan pools respectively. The superscripts refer to the case study - diff for diffusion and ing for ingestion, whereas the subscripts refer to the time step number $n$. The other parameters are: $\mathrm{k}_{\mathrm{dep}}$ - bacterial depuration rate constant $\left(\min ^{-1}\right), \mathrm{IR}$ - ingestion rate (cells/min), $\mathrm{k}_{\text {for }}$ - protozoan uptake rate constant $\left(\mathrm{min}^{-1}\right), \mathrm{k}_{\mathrm{rev}}-$ protozoan loss rate constant $\left((\mathrm{g} \mathrm{OC})^{-1} \mathrm{~min}^{-1}\right),[\mathrm{P}]$ - protozoan concentration (cells $/ \mathrm{mL}$ ) and $[\mathrm{OC}]_{\mathrm{p}}$ - organic carbon per protozoan (gOC/cell). Equations 15 and 22 are used for grazing protozoa only (ingestion case study).

The model was run with the protozoan rate constants derived from the regression analysis of the radioactive uptake experiment (sample model run shown in Figure 2-9). The total activity of the model system was $100 \mathrm{dpm}$ (in a $1 \mathrm{~mL}$ system). As written, the model does not include uptake into the bacteria. If alternate values are used for a bacterial uptake rate constant $\left(10^{*} \mathrm{k}_{\text {dep }}, 2 * \mathrm{k}_{\text {dep }}\right.$, and $\left.0.1^{*} \mathrm{k}_{\text {dep }}\right)$, the relative amounts of PCBs in the bacterial and protozoan pools change but the time required for equilibration does not $(<15 \mathrm{~min})$. The addition of ingestive uptake of PCBs does not change any part of the model run, neither the mass of PCBs within the protozoan pool nor the time to equilibration. This result is consistent with the stable and radioactive bioaccumulation experiments. 


\subsection{Discussion}

The equivalence of BCFs in both the diffusion and ingestion flasks is a compelling piece of evidence in this study that diffusion is the primary method of $\mathrm{CB}$ uptake for the model ciliate. This conclusion was further bolstered by the subsequent radioactive experiments in which three replicate trials of $\mathrm{CB} \# 77$ uptake into the model ciliate exhibited pseudo-first order kinetics with respect to the aqueous CB concentration. This study shows that equilibrium between the different organic carbon pools in this system is achieved quickly. and diffusion dominates CB uptake for protozoa $10 \mu \mathrm{m}$ or smaller. The role of DOC in these cultures may be to sequester large chlorine compounds and render them relatively "unavailable" for volatilization (see Figure 2-1) or biological uptake (see Figure 2-5) - both a function of the truly dissolved CB concentration. The addition of ingested CB-DOC complexes did not allow ingestion to out-compete diffusion as the primary mode of uptake in these organisms. The results of the numerical model were consistent with this hypothesis in that equilibration occurred quickly ( $<10 \mathrm{~min})$. The addition of DOC as a complexing agent for the PCBs only changed the thermodynamically-controlled equilibrium concentration, not the rate of uptake into the protozoan cell.

These results could be extended to other prey species such as cyanobacteria or phytoplankton. Differences in cell composition among these various species will affect prey $\mathrm{CB}$ concentrations. However, the difference between the diffusive and ingestion uptake rates is so large that small variations in the prey $\mathrm{CB}$ concentrations should not affect the general conclusions of this study. Changes in prey species will also influence protozoan clearance rates. Again, unless the increase in clearance rates is a factor of 10

or higher, diffusion will still out-compete ingestion and the conclusions of this study will remain unchanged.

The conclusion of this study has implications for the prediction of uptake pathways in other organisms. We, in addition to other investigators, have shown that diffusion dominates uptake in extremely small organisms. Trophic transfer studies have 
shown that ingestion dominates CB uptake in macroscopic organisms (Rubinstein et al., 1984). Therefore, there must be a transition in the size spectrum of organisms between diffusion- and ingestion-dominated $\mathrm{CB}$ uptake. We can estimate this transitional size by comparing the ratio between diffusion and ingestion for species within a phylum. This comparison allows all parameters in equation 6 to be held constant except those relating to cell size and ingestion and/or clearance rates. This assumes that cellular membrane characteristics do not change across the organism size spectrum. The transitional size is dependent to a certain extent on the congener chosen in that the diffusion coefficient through the cellular membrane is a function of molecular weight, according to equation 6 presented in the introduction. The partition coefficient, $\mathrm{K}_{\mathrm{Iw}}$, was cancelled from the equation because it was present in both the numerator and denominator of equation 6 .

The transitional size where uptake via diffusion and ingestion are equivalent was estimated in two ways: first by varying feeding rates in a series of ciliate species and second, by varying clearance rates and optimal prey concentrations for the same series of species. In the first case, I have used feeding rates for a number of ciliate species ranging from 4-400 $\mu \mathrm{m}$ in diameter from Fenchel (1980) and substituted them into the relationship between maximum ingestion rate and cell size from Figure 2 of Fenchel (1980): $I R_{\max }=$ $2.78 \mathrm{E}-4 * \mathrm{Vol}^{0.85}$ - where IR is ingestion rate $\left(\mathrm{m}^{3} / \mathrm{s}\right)$ and $\mathrm{Vol}$ is cell volume $\left(\mathrm{m}^{3}\right)$. After substituting maximum ingestion rates for $\mathrm{CR}^{*} \mathrm{BC} * \mathrm{BV}$ in equation 6 , the ratio between diffusion and ingestion reduces to a function of the cell radius: $0.00834 r^{-0.55}$. From this relationship, the cell radius at which diffusion and ingestion are equal is approximately $166 \mu \mathrm{m}$, corresponding to a cell diameter of $332 \mu \mathrm{m}$. Using molecular diffusion coefficients for a lower chlorinated congener and a higher chlorinated congener, I get the following values for the transitional size. For IUPAC\#28 ( $3 \mathrm{Cl}$ 's), $M W=184$, $\mathrm{D}_{\mathrm{m}}=1.08 \times 10^{-16} \mathrm{~m}^{2} / \mathrm{s}, \mathrm{r}=193 \mu \mathrm{m}$ and cell diameter is $386 \mu \mathrm{m}$. For IUPAC\#180 (7 Cl's), $\mathrm{MW}=320, \mathrm{D}_{\mathrm{m}}=8.3 \times 10^{-17} \mathrm{~m}^{2} / \mathrm{s}, \mathrm{r}=117 \mu \mathrm{m}$ and cell diameter is $234 \mu \mathrm{m}$.

To obtain an independent estimate, maximum clearance rates (CR) and optimal prey concentrations (BC*BV) for each species studied by Fenchel (1980) can be employed in a similar manner as above. In this estimate, diffusion and ingestion are 
equivalent at approximately $50 \mu \mathrm{m}$ cell radius or $100 \mu \mathrm{m}$ cell diameter. This second estimate is consistent with the field data of Axelman et al. (1997). Their data showed that particulate $\mathrm{CB}$ concentrations in the $2-20 \mu \mathrm{m}$ size fractions and smaller were in equilibrium with dissolved PCBs. Particles in the $20-200 \mu \mathrm{m}$ size fraction and larger had lower $\mathrm{CB}$ concentrations than predicted from equilibrium calculations. These data suggested that diffusion is not occurring fast enough to allow full equilibration of the larger size class with the surrounding aqueous environment.

Both calculations presented above over-estimate the importance of diffusion because certain limitations were not taken into account. After PCBs are incorporated into the cellular membrane, they are transported to other cellular compartments by diffusion and/or internal mixing. In the example calculation presented in the introduction, the cellular mixing rate was assumed to be practically instantaneous such that the ratelimiting step for cellular CB uptake was transport through the phospholipid membrane. As cell size increases, mixing within the cell will play a larger role in the overall equilibration with aqueous PCBs. The addition of cellular mixing as a rate-limiting step will increasingly lengthen the time for diffusive equilibration with increasing cell radius.

Full equilibration with internal cellular compartments will be further inhibited by cellular growth and the addition of new biomass, most noticeably in larger cells. This phenomenon was not observed in the presented laboratory cultures, but it is possible that the surface area to volume ratio of the model ciliate was too large. However, biomass dilution was observed in algal cultures by Swackhamer et al. (1993). Since the algae (20$30 \mu \mathrm{m})$ are not capable of ingestion of CB-laden particles, they are dependent on diffusion as an uptake mechanism and thus are affected by the surface area to volume dependence of diffusive equilibration. While my calculations predict diffusive equilibrium for organisms in this size range, it is possible that these organisms are large enough to be affected by internal equilibration barriers, given the uncertainties in some of the parameters.

Lastly, there is no attempt in the above calculation to address the effect of composition of the cellular surface or increased surface area due to the presence of 
frustules (e.g., diatoms) or reticulopodia (e.g., foraminifera and radiolaria). These morphological features are composed of materials that are lipid-poor and thus should have much lower affinity for PCBs than phospholipid bilayers. However, the increase in surface area should increase the relative contribution of diffusion to $\mathrm{CB}$ uptake. The overall effect of these counter-balancing parameters will be species-dependent. Being mindful of the limitations of these calculations, the best estimate at this time for the transitional size where diffusion is approximately equal to ingestion is $50-150 \mu \mathrm{m}$ cell radius or $100-300 \mu \mathrm{m}$ cell diameter.

\subsection{Conclusions}

In summary, these experimental data support the hypothesis that uptake of PCBs by a model protozoan species is dominated by passive diffusion across the cellular membrane. Equilibrium with the surrounding environment is achieved very quickly $(<1$ hour). Organic carbon content determines the steady-state (6h) internal CB concentration as shown by the bioconcentration factors. Independently determined rate constants for protozoan uptake and bacterial depuration were inserted into a numerical model. Comparison of ingestion and diffusion uptake rates using this numerical model corroborated the hypothesis that diffusion is dominant for our target organism. 


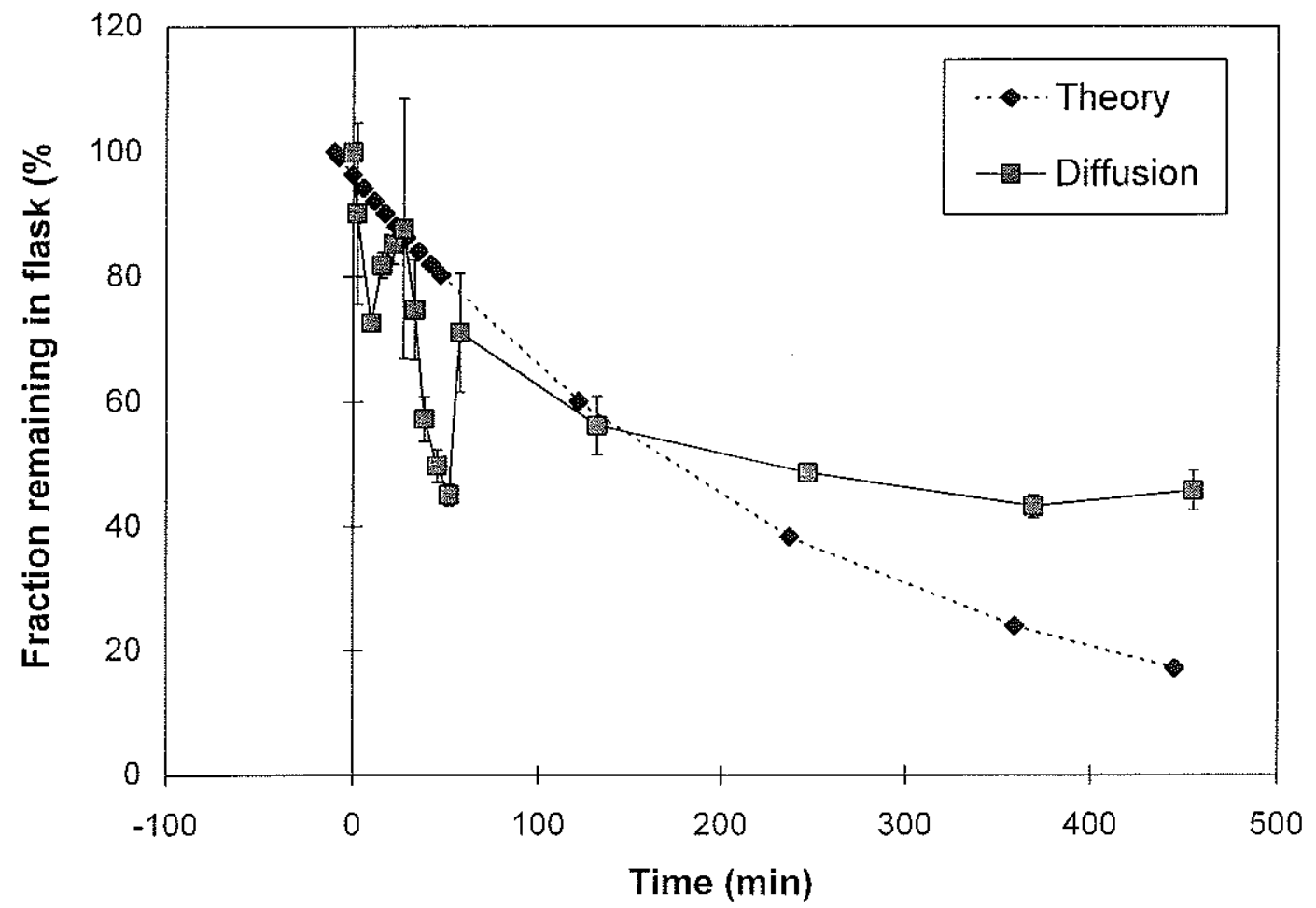

Figure 2-1. Total recovery for IUPAC \#187 versus volatilization model.

Total recoveries for IUPAC \#187 from the prey-limited (or diffusion) flask are plotted versus time (squares). A model curve showing the effect of volatilization is also plotted (diamonds). The model data were generated using a nominal wind speed of $1 \mathrm{~m} / \mathrm{s}$ and the volatilization was begun 10 minutes prior to the start of the experiment. 

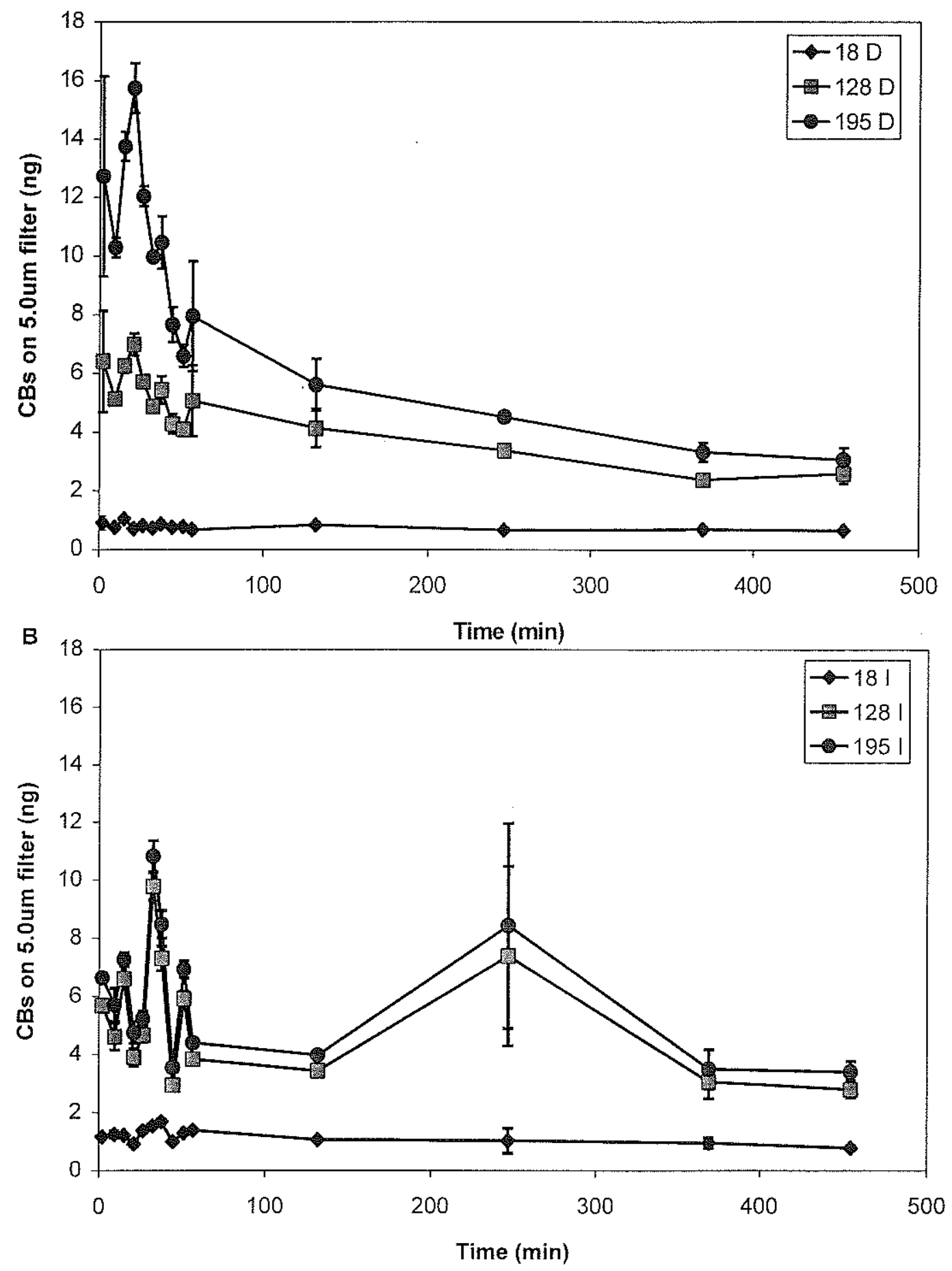

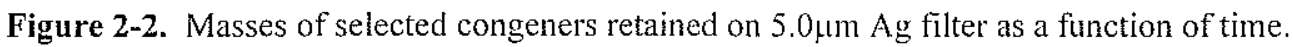
Diffusion flask replicate 1 (A) and ingestion flask replicate 1 (B). Congeners plotted: IUPAC \#I 8 (diamonds) $-\log \mathrm{K}_{\mathrm{ow}}=5.24$; IUPAC \#128 (squares) - $\log \mathrm{K}_{\mathrm{ow}}=6.74 ;$ IUPAC \#195 (circles) $-\log \mathrm{K}_{\mathrm{ow}}=$ 7.56. Masses are the average of determinations using two internal recovery standards (see text). Errors are \pm 1 standard deviation of these averages. 

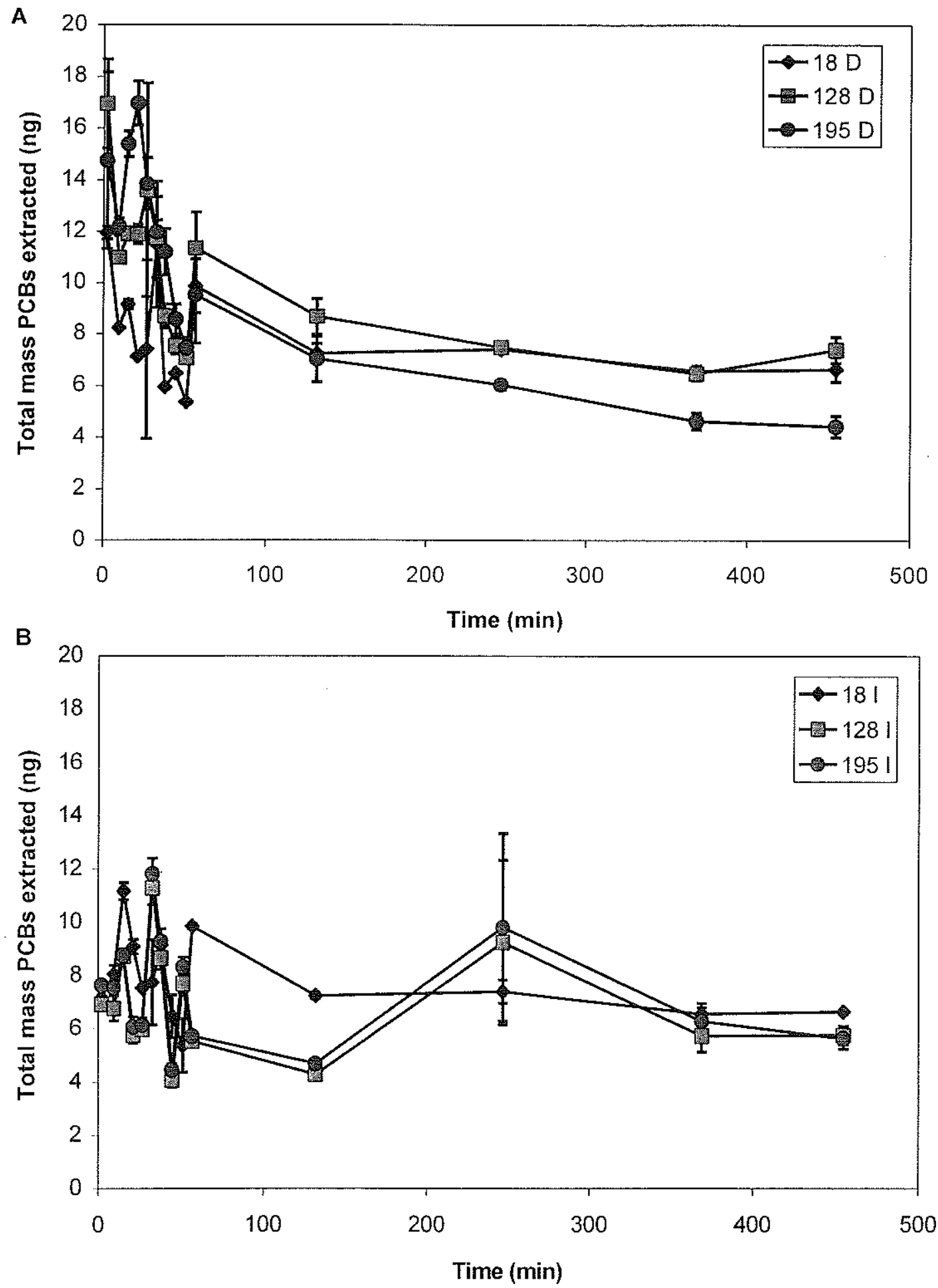

Figure 2-3. Total PCBs extracted per sample.

Diffusion flask replicate $1(\mathbf{A})$ and ingestion flask replicate $1(\mathbf{B})$. The same congeners are plotted as in Figure 2-2. The initial $\mathrm{CB}$ addition was $15,000 \mathrm{pg}$ per congener per $40 \mathrm{~mL}$ in each flask. 

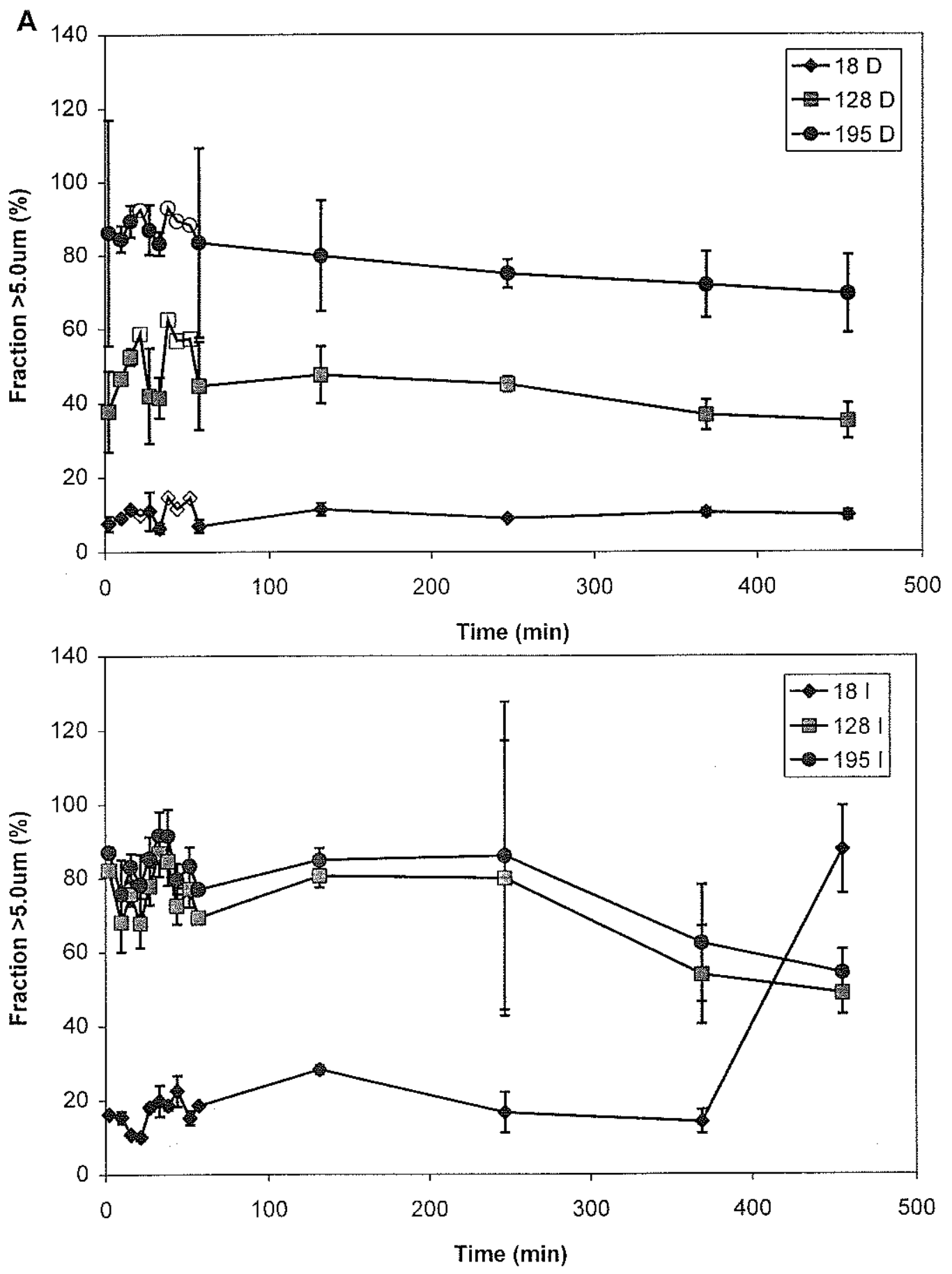

Figure 2-4. Fraction of three selected congeners $(18,128,195)$ retained on $5.0 \mu \mathrm{m}$ filter vs. time. Diffusion flask replicate $1(\mathbf{A})$ and ingestion flask replicate $1(\mathbf{B})$. Percent $=$ (Mass on $5.0 \mu \mathrm{m}$ filter $) /($ Mass on $5.0 \mu \mathrm{m}$ filter + Mass in $5.0 \mu \mathrm{m}$ filtrate $)^{*} 100 \%$. Errors were propagated from both filter and filtrate extractions. Open symbols are used for samples in which one of the recovery standards in the filtrate was too low and only one recovery standard could be used to estimate CB content. 
- Ingestion Diffusion $\triangle \mathrm{Log} \mathrm{Koc}$

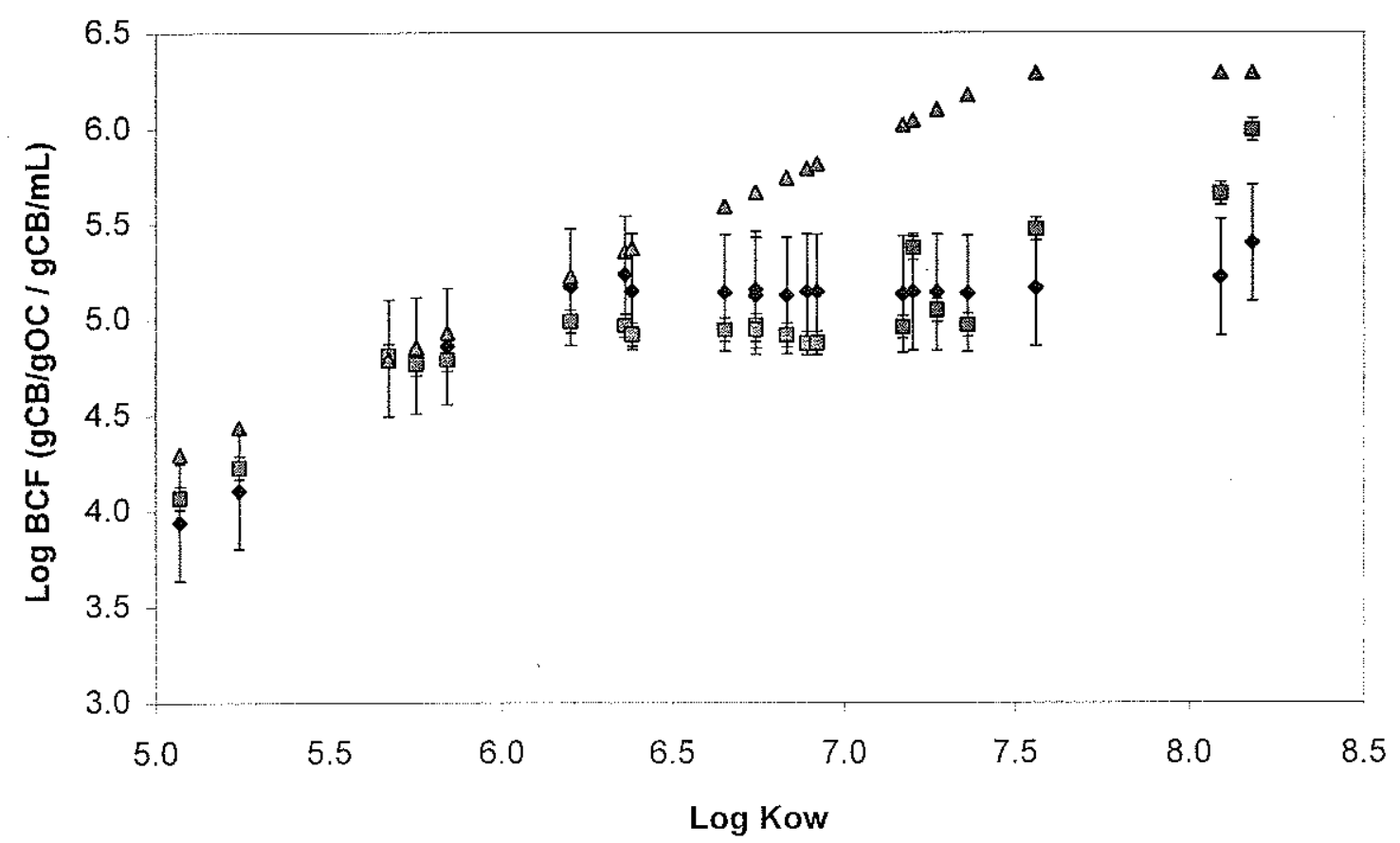

Figure 2-5. Bioconcentration factors for each congener in the experimental flasks.

$\mathrm{BCF}=(\mathrm{CB}$ in $>5.0 \mu \mathrm{m}$ fraction, normalized to organic carbon $) /(\mathrm{CB}$ in $<0.2 \mu \mathrm{m}$ filtrate $)$. Errors were propagated from errors on $\mathrm{CB}$ and organic carbon analyses. Each $\mathrm{BCF}$ is plotted versus the hydrophobicity of the congener, $\log \mathrm{K}_{\mathrm{ow}}$. Diffusion flask data are indicated by squares and ingestion flask data are indicated by diamonds. Triangles indicate $\mathrm{K}_{\mathrm{oc}}$ values as predicted from the relationship in Schwarzenbach et al. (1993): $\log \mathrm{K}_{\mathrm{oc}}=0.82 * \log \mathrm{K}_{\mathrm{ov}}+0.14$. This relationship was derived for compounds with $\log \mathrm{K}_{\mathrm{ow}}$ up to 7 (see Figure 11.10 in Schwarzenbach et al. (1993)). Linear relationships have been shown for congeners with $\log \mathrm{K}_{\mathrm{ow}}$ up to 7.5 (Bergen et al., 1996). For congeners with $\log \mathrm{K}_{\mathrm{ow}}>7.5, \log \mathrm{K}_{\mathrm{oc}}$ is kept constant at 6.3 (equals $0.82 * 7.5+0.14$ ). 
A
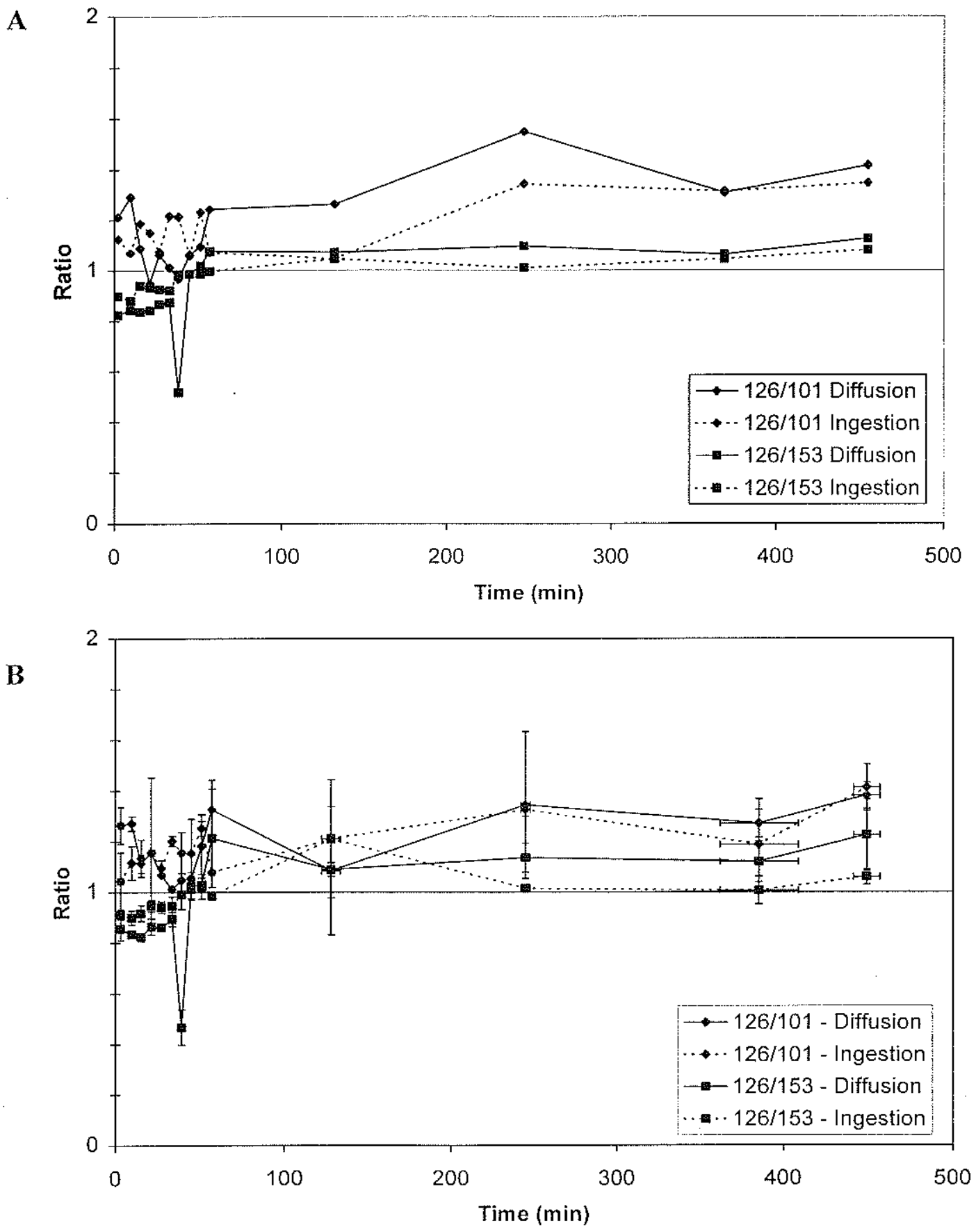

Figure 2-6. Ratio of coplanar to non-coplanar PCBs in bioaccumulation experiment.

Two ratios of a non-coplanar to a coplanar CB congener are shown. IUPAC \#126 is a coplanar CB with 5 chlorines ( $\log \mathrm{K}_{\mathrm{ow}}=6.89$ ) and both IUPAC \#10 land IUPAC \#153 are non-coplanar CB congeners with 5 chlorines ( $\log \mathrm{K}_{\mathrm{ow}}=6.38$ and 6.92 , respectively). The ratio of \#126 to \#101 is designated by diamonds (diffusion = solid line and ingestion = dotted line) and the ratio of \#126 to \#153 is designated by squares. A: Data from replicate I only. B: Data from both replicates and past experiments. Error bars are $\pm 1 \sigma$ of the mean of the ratios at specific time points. 


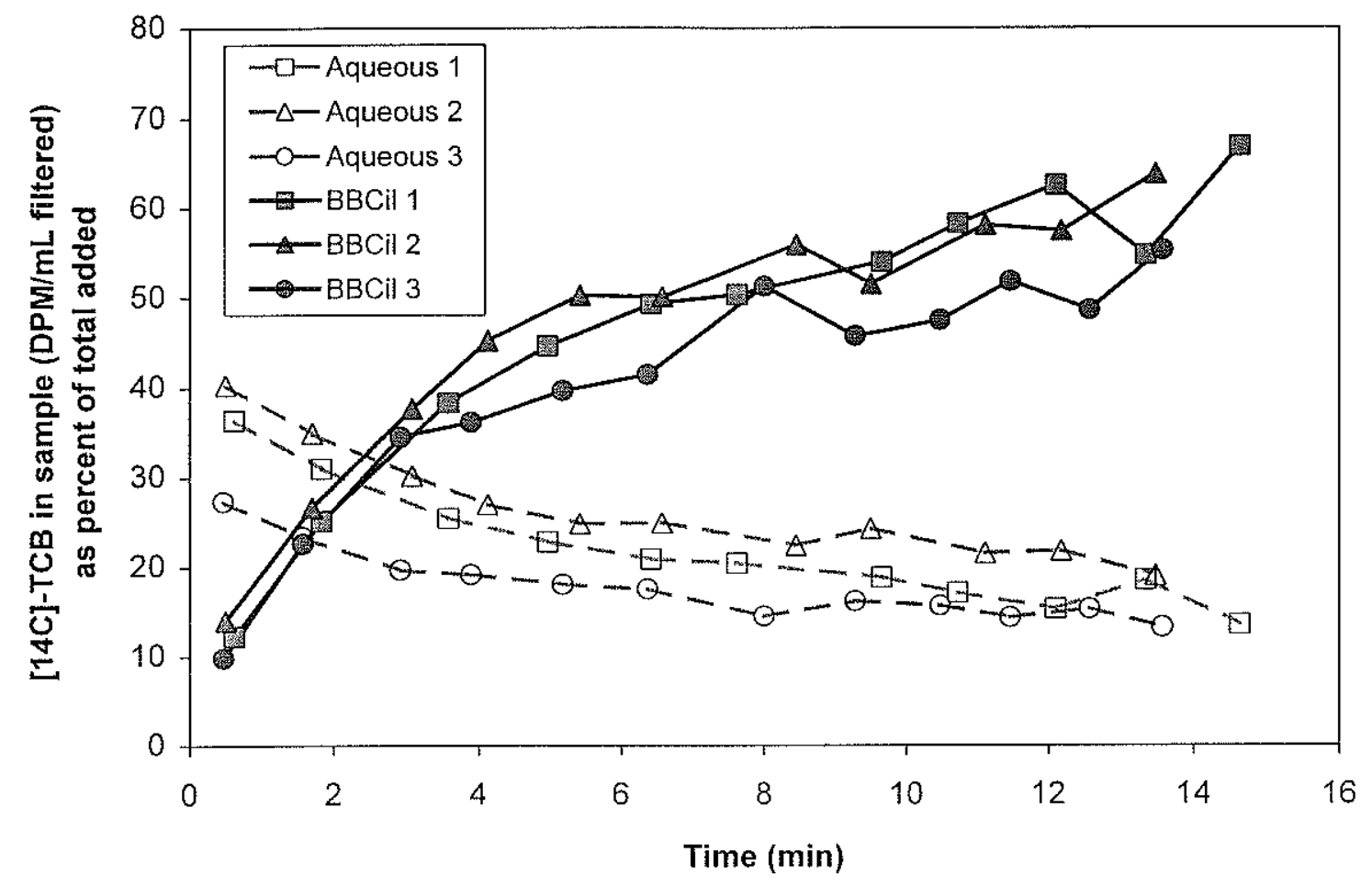

Figure 2-7. Radioactive bioaccumulation experiment.

$50 \mathrm{~mL}$ of a protozoan culture was filtered through a $5.0 \mu \mathrm{m}$ filter for each time point (solid lines). Three replicate experiments are shown. Aqueous CB concentrations are also shown as a function of time for three replicates (dashed lines) and are corrected for PCBs associated with DOC. Organic carbon concentrations used for each replicate were: Expt \#1 - 5.62mg/L; Expt \#2 - 4.63mg/L; Expt \#3$9.16 \mathrm{mg} / \mathrm{L}$. 


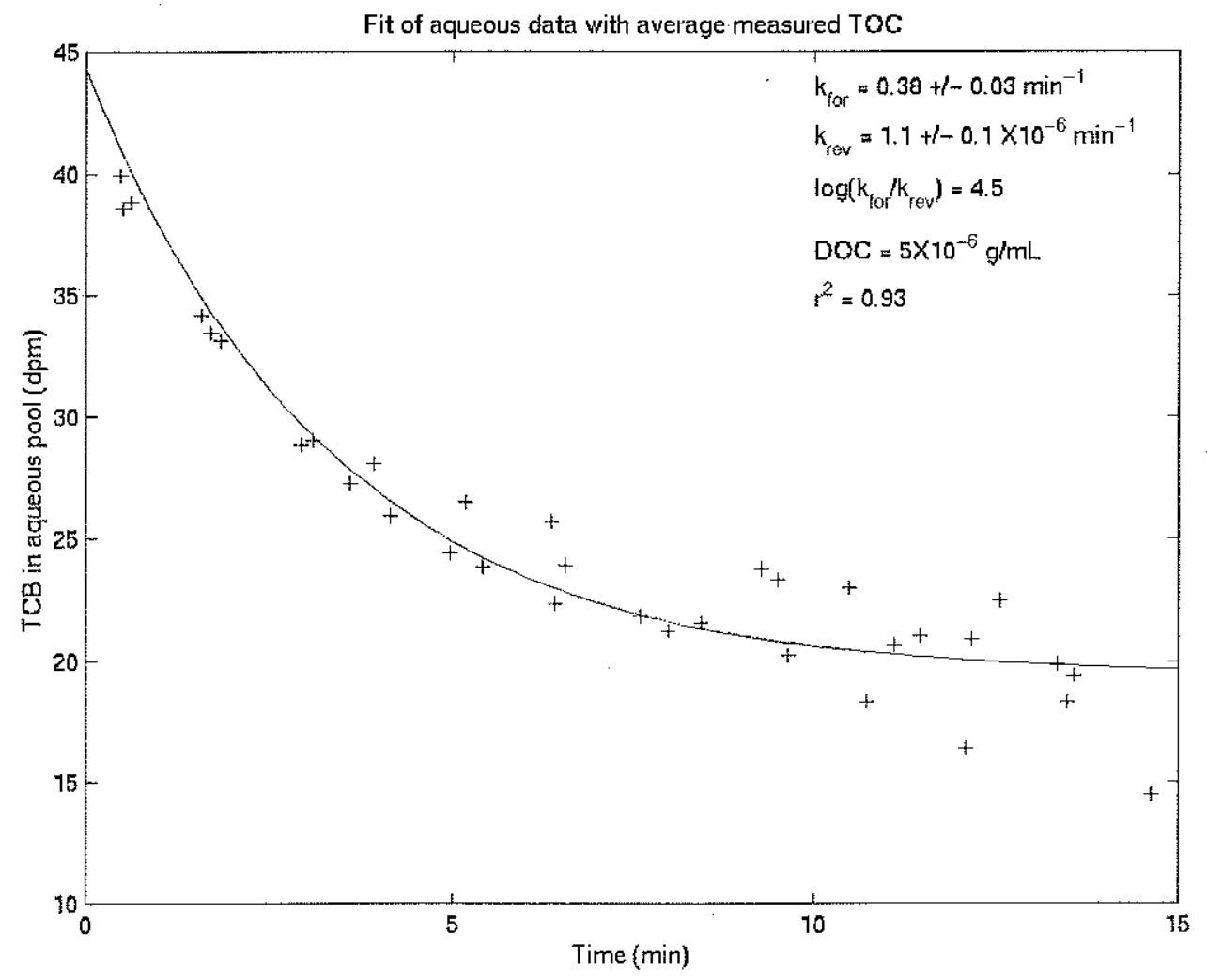

Figure 2-8. Non-linear regression fits for data from radioactive experiment.

Data points are ${ }^{14} \mathrm{C}-\mathrm{TCB}$ aqueous concentrations as calculated from DOC concentrations and ${ }^{14} \mathrm{C}-\mathrm{TCB}$ $(>5,0 \mu \mathrm{m})$. Average $[\mathrm{DOC}]=5 \times 10^{-6} \mathrm{~g} / \mathrm{mL}$. The aqueous pool refers to the truly dissolved $\mathrm{CB}$ concentration. The analytical solution derived in the text was fit to the radioactive data using the Levenberg-Marquardt Method of non-linear regression. 


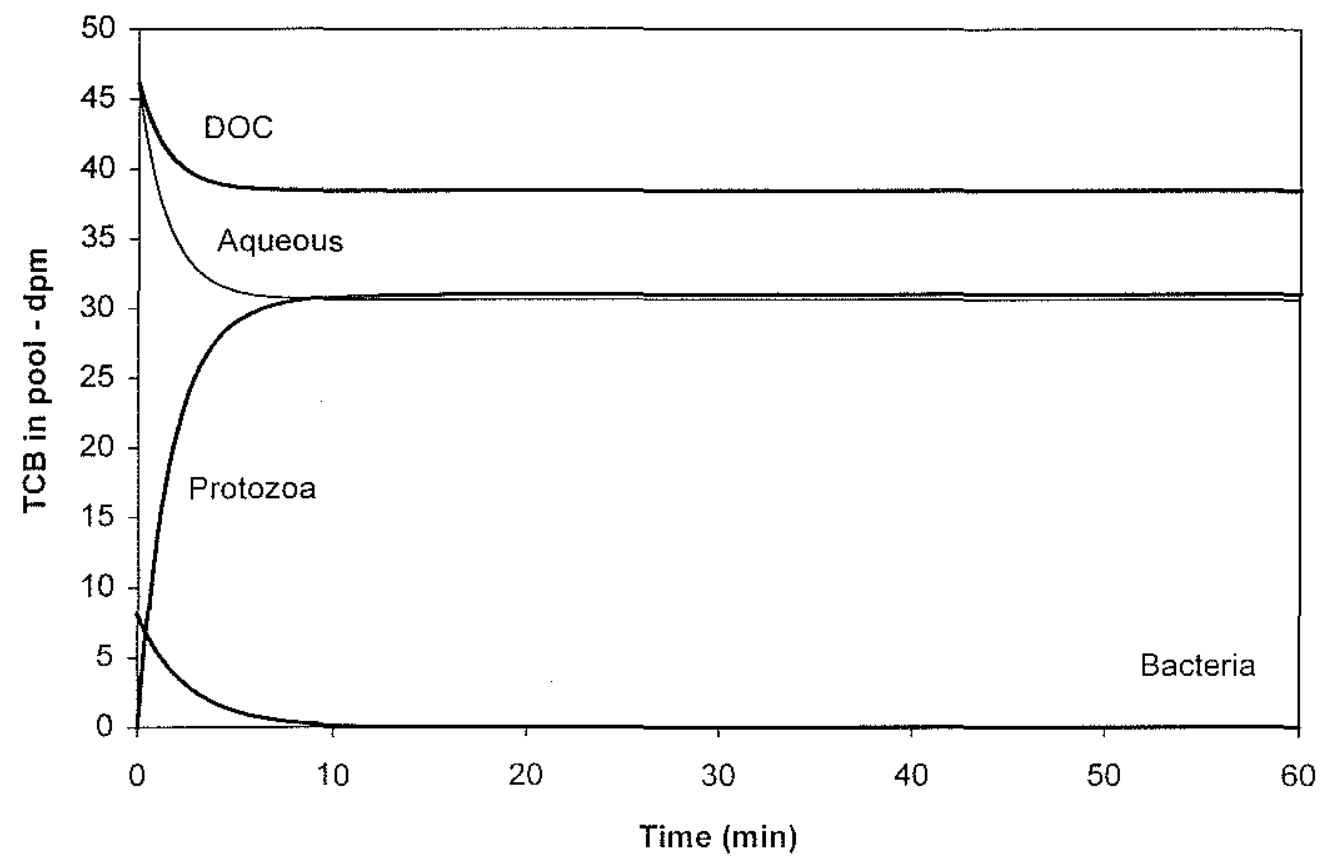

Figure 2-9. Model runs using parameters from radioactive experiments.

Total $\mathrm{CB}=100 \mathrm{dpm}(/ \mathrm{mL})$. Initial conditions: [Protozoa] $=10^{4} \mathrm{cells} / \mathrm{mL} ;$ [Bacteria] $=10^{7} \mathrm{cells} / \mathrm{mL}$;

$[D O C]=4 \times 10^{-6} \mathrm{~g} / \mathrm{mL}$; Ingestion rate $=0.56 \mathrm{cell} \mathrm{s} / \mathrm{min} ; \mathrm{CB}$ in bacterial cells $=8.06 \mathrm{dpm} ; \mathrm{CB}$ in aqueous phase $=45.87 \mathrm{dpm} ; C B s$ in DOC pool $=46.07 \mathrm{dpm} ; \mathrm{k}_{\mathrm{for}}=0.38 \mathrm{~min}^{-1} ; \mathrm{k}_{\mathrm{rev}}=1.1 \times 10^{-5}(\mathrm{~g} \mathrm{OC})^{-1} \mathrm{~min}^{-1}$. 


\section{Evidence for DOC-enhanced molecular diffusion in a bacterial culture}

\subsection{Introduction}

Polychlorinated biphenyls (PCBs) are hydrophobic lipophilic compounds that bioaccumulate in the fatty and lipid tissues of higher organisms. These compounds have been shown to pose a health risk to a number of marine organisms including fish and mammals. Large organisms are often found to acquire PCBs primarily through ingestion of contaminated foodstuffs (Rubinstein et al., 1984). It is important to look at the base of the food chain to understand how PCBs begin their journey to higher organisms. In many aquatic ecosystems, the microbial loop is the base of the food chain. The time scale necessary for equilibration of the microbial loop with its aqueous surroundings is a key parameter in predicting both the magnitude and rate of chlorobiphenyl (CB) accumulation in the food chain.

The microbial loop consists of unicellular organisms such as phytoplankton, bacteria, and protists, primarily nano- and micro-zooplankton. Phagotrophic protozoa rely on the ingestion of bacteria and small phytoplankton for nutrition. However, as presented in Chapter 2, this study suggests that they accumulate PCBs primarily through diffusion. In diffusion dominated systems, the maximum concentration of PCBs can be predicted from $\mathrm{K}_{\mathrm{oc}}$, the organic carbon-water partition coefficient. The time needed to achieve this maximal concentration will vary according to the aqueous $\mathrm{CB}$ concentration and the uptake and depuration rate constants for organisms within this microbial loop. The combination of uptake and depuration rates among the different organism classes will determine how quickly the system reaches equilibrium with aqueous PCBs.

The rate constants describing uptake of $\mathrm{PCBs}$ by protozoa were experimentally determined in the previous chapter. The $\mathrm{CB}$ uptake rate constants in the absence of appreciable bacteria were assumed to be indicative of pure diffusive uptake. While this conclusion is robust within the inherent variability of the experiment, there are a number of complications that should be addressed. Obviously, protozoa are living, biological 
"particles". This means that they're constantly swimming in their aqueous environment and seeking food particles. When food concentrations are low, fewer food vacuoles are produced. However, these food vacuoles will contain both prey-associated PCBs and dissolved organic carbon (DOC)-associated PCBs. In addition, the ciliates examined in the previous chapter are covered by small cilia. The effect of all these parameters on the diffusive boundary layer surrounding the protist and the subsequent measured diffusive uptake rate constant is difficult to ascertain. It is clear from the previous chapter that ingestion of contaminated bacteria cannot supply PCBs to the protist faster than diffusion. However, the loss rate constants from bacteria and CB-DOC complexes should be measured to be sure that this process can supply the CBs needed for protist uptake.

The role of dissolved organic carbon (DOC) was not fully addressed in the previous chapter. DOC concentrations were not determined and so the extent to which PCBs were associated with DOC could not be calculated. In addition, the role of DOC in the transfer of PCBs from bacteria to protists was not considered. Recent evidence suggested that organic material (e.g., bile salt micelles) could enhance the diffusive transport of PCBs in the diffusive boundary layer of intestines (Dulfer et al., 1996; Dulfer et al., 1998). The effect of DOC-enhanced diffusion will be a kinetic one. The equilibrium concentration within an organism (or particle) will be determined by the physico-chemical parameters of the $\mathrm{CB}$ congener and the organic carbon content and composition of all organic phases within the system. This value is independent of rate or method of uptake. The approach to this equilibrium value is a function of the diffusive boundary layer surrounding the "particle", the molecular diffusion coefficient of the compound of interest, and the equilibrium partition coefficient. If DOC is playing a role in aiding molecular diffusion, it will shorten the time needed to achieve equilibrium with the "particulate" phase.

In this paper, I present the results of a study designed to measure the CB loss rate constant from bacteria and the disassociation rate constant of CB-DOC complexes. The extraction method employed, extraction of dissolved PCBs by Tenax resin, was slower 
than the loss rate constant of PCBs from bacteria. Therefore, a lower limit of this loss rate constant is presented. In addition, interactions between PCBs and DOC were observed that proved useful in extending our understanding of the role of $\mathrm{DOC}$ in $\mathrm{CB}$ diffusion. It is these latter observations that will be the focus of this chapter.

Tenax resin beads were added to a slurry of bacteria and DOC in seawater. The extraction of the aqueous PCBs in the presence of dissolved organic material was compared to that in seawater. The experiment was performed twice (in triplicate). From the difference in extraction rate constants, a lower limit for the CB-DOC disassociation rate constant was calculated. These results suggest that DOC-enhanced diffusion is occurring and increases the extraction rate constant by a factor of two or more for the radioactive congener used (IUPAC \#77). Results were extended for the remaining 12 congeners. DOC-enhanced diffusion was important for all congeners studied.

\subsection{Methods}

\subsubsection{Growth of organisms.}

Vineyard Sound seawater (VSW) was used for all experiments presented in this chapter and was collected and treated in the same manner as described in Chapter 2. This batch of seawater was collected in the spring of 1999. The bacteria used in this experiment were Halomonas halodurans, a ubiquitous $0.45 \mu \mathrm{m}$ marine bacterium from the collection of D. Caron, University of Southern California (USC), CA. The bacterium was grown on $0.04 \%(\mathrm{w} / \mathrm{w})$ yeast extract in $0.2 \mu \mathrm{m}$-filtered, sterile Vineyard Sound seawater (VSW). When the cells had reached stationary phase (12-15 hours), they were harvested with centrifugation. Aliquots of the culture $(40 \mathrm{~mL})$ were centrifuged at $10,000 \mathrm{rpm}(11,180 \mathrm{Xg})$ and $15^{\circ} \mathrm{C}$ for $23 \mathrm{~min}$ and resuspended in sterile VSW (Biofuge $22 \mathrm{R}$, Heraeus). The bacterial pellet was resuspended in one-half the initial volume. This was repeated twice (three times total) to ensure complete removal of excess yeast extract. The bacterial concentrate for this experiment contained $5.5 \times 10^{8}$ cells $/ \mathrm{mL}$. 


\subsubsection{Polychlorinated biphenyls.}

The PCBs used in this experiment were purchased from AccuStandard (Cat\# CCCSEC-R, Lot \#124-269, New Haven, CT) as a mixture of 21 congeners (approximately $100 \mu \mathrm{g} / \mathrm{mL}$ per congener in acetone) spanning the range of hydrophobicities (see Table 3 1). In addition, solutions of $\mathrm{CB} \# 47,2,2^{\prime}, 4,4^{\prime}$-tetrachlorobiphenyl, and $\mathrm{CB} \# 169$, 3,3',4,4',5,5'-hexachlorobiphenyl were purchased from AccuStandard (Lot \#'s A7090166 and 086-108, respectively, both approximately $35 \mu \mathrm{g} / \mathrm{mL}$ in iso-octane). All three solutions from AccuStandard were diluted with acetone to approximately $4 \mathrm{~mL}$. Their concentrations at the time of the experiment were $24.2 \mu \mathrm{g} / \mathrm{mL}$ ( $\mathrm{CB}$ mix), $8.3 \mu \mathrm{g} / \mathrm{mL}$ (\#47) and $11.5 \mu \mathrm{g} / \mathrm{mL}$ (\#169).

\begin{tabular}{|c|c|c|}
\hline $\begin{array}{c}\text { Congener \# } \\
\text { (IUPAC) }\end{array}$ & Structure & $\log K_{0 w}$ \\
\hline 8 & $2,4^{\prime}$-dichlorobiphenyl & 5.07 \\
\hline 18 & $2,2^{\prime}, 5$ & 5.24 \\
\hline 28 & $2,4,4^{\prime}-$ & 5.67 \\
\hline 44 & $2,2^{\prime}, 3,3^{\prime}-$ & 5.75 \\
\hline 47 & $2,2^{\prime}, 4,4^{\prime}-$ & 5.85 \\
\hline 52 & $2,2^{\prime}, 5,5^{\prime}-$ & 5.84 \\
\hline 66 & $2,3^{\prime}, 4,4^{\prime}-$ & 6.2 \\
\hline 77 & $3,3^{\prime}, 4,4^{\prime}-$ & 6.36 \\
\hline 101 & $2,2^{\prime}, 4,5,5^{\prime}-$ & 6.38 \\
\hline 105 & $2,3,3^{\prime}, 4,4^{\prime}-$ & 6.65 \\
\hline 118 & $2,3^{\prime}, 4,4^{\prime}, 5-$ & 6.74 \\
\hline 126 & $3,3^{\prime}, 4,4^{\prime}, 5-$ & 6.89 \\
\hline 128 & $2,2^{\prime}, 3,3^{\prime}, 4,4^{\prime}-$ & 6.74 \\
\hline 138 & $2,2^{\prime}, 3,4,4^{\prime}, 5^{\prime}-$ & 6.83 \\
\hline 153 & $2,2^{\prime}, 4,4^{\prime}, 5,5^{\prime}-$ & 6.92 \\
\hline 169 & $3,3^{\prime}, 4,4^{\prime}, 5,5^{\prime}-$ & 7.42 \\
\hline 170 & $2,2^{\prime}, 3,3^{\prime}, 4,4^{\prime}, 5-$ & 7.27 \\
\hline 180 & $2,2^{\prime}, 3,4,4^{\prime}, 5,5^{\prime}-$ & 7.36 \\
\hline 187 & $2,2^{\prime}, 3,4^{\prime}, 5,5^{\prime}, 6-$ & 7.17 \\
\hline 195 & $2,2^{\prime}, 3,3^{\prime}, 4,4^{\prime}, 5,6-$ & 7.56 \\
\hline 199 & $2,2^{\prime}, 3,3^{\prime}, 4,5,6,6^{\prime}-$ & 7.2 \\
\hline 206 & $2,2^{\prime}, 3,3^{\prime}, 4,4^{\prime}, 5,5^{\prime}, 6-$ & 8.09 \\
\hline 209 & Decachlorobiphenyl & 8.18 \\
\hline
\end{tabular}

Table 3-1. Congeners used in this study - structures and $\log \mathrm{K}_{\text {ow }}$. Log $\mathrm{K}_{\mathrm{ow}}$ values from Hawker and Connell (1988). 


\subsubsection{Experimental protocol.}

These experiments are based on the method published by Cornelissen et al. (1997). [Note: During the data analysis for this chapter, a mistake was found in the data interpretation equation used in Cornelissen et al. (1997). The corrected solution (and its derivation) is presented in Appendix C.] As in the Cornelissen et al. (1997) study, Tenax resin (Tenax TA polymer (porous polymer based on 2,6-diphenyl-p-phenylene oxide), $60 / 80$ mesh, 177-250 $\mu \mathrm{m}$ bead size - Supelco, Belafonte, PA) was used to extract aqueous PCBs from the experimental solution. Tenax resin was originally chosen by Pignatello (1990) because the sorption capacity of this polymer was similar to that of soil organic carbon and it was effective at keeping the aqueous concentration of a hydrophobic compound very low.

Control experiments were conducted with sterile VSW to determine the ${ }^{14} \mathrm{C}-\mathrm{TCB}$ (3,3',4,4'-tetrachlorinated biphenyl - IUPAC \#77) uptake rate constant of the Tenax resin. Three separatory funnels were used for the control experiment. Each funnel contained $100 \mathrm{~mL}$ VSW inoculated with ${ }^{14} \mathrm{C}-\mathrm{TCB}$ (concentration $=0.35 \mu \mathrm{g} / \mathrm{mL}$, approximately $140 \mathrm{dpm} / \mathrm{mL}$ ). After addition of TCB, each funnel was shaken well for $15 \mathrm{sec}$ and then left to sit for approximately $30 \mathrm{~min}$. Prior to the addition of Tenax, three $7 \mathrm{~mL}$ aliquots were removed from each funnel to determine the initial ${ }^{14} \mathrm{C}-\mathrm{TCB}$ concentration. When Tenax resin $(10 \mathrm{mg})$ was added to each separatory funnel, the resin dispersed homogenously in the solution. No evidence of aggregation or clumping was observed. After Tenax addition, the funnel was shaken by hand for $5 \mathrm{~min}$. Once the Tenax resin floated to the top of the funnel, the VSW was transferred to an Erlenmeyer flask while the Tenax resin clung to the walls of the funnel. Three $7 \mathrm{~mL}$ aliquots of the VSW were removed to determine the remaining ${ }^{14} \mathrm{C}-\mathrm{TCB}$ concentration. The residual Tenax resin was rinsed once with Milli-Q water and once with $10 \mathrm{~mL}$ hexane.

The experiment was repeated with a bacterial suspension. This bacterial suspension was incubated with a mix of $\mathrm{CB}$ congeners for approximately $2 \mathrm{~h}$ and $5 \mathrm{~h}$. The bacterial concentrate described above (section 3.2.1.) was diluted with sterile VSW to a concentration of $3.7 \times 10^{7}$ bacteria $/ \mathrm{mL}$ in $700 \mathrm{~mL}$. To this bacterial suspension was 
added $9 \mu \mathrm{L}$ of CB mix stock, $25 \mu \mathrm{L}$ of \#47 stock, and $18 \mu \mathrm{L}$ of \#169 stock, giving a total concentration of $0.3 \mathrm{ng} / \mathrm{mL}$ per congener. After approximately 2 hours, three $100 \mathrm{~mL}$ aliquots of the CB-labeled bacterial suspension were transferred to $125 \mathrm{~mL}$ separatory funnels. A $20 \mathrm{~mL}$ aliquot of the suspension was removed from each funnel for initial CB concentrations. Tenax resin (10mg) was then added to each funnel. Due to the impossibility of shaking (by hand) all three funnels at once, the funnels were shaken intermittently for approximately $5 \mathrm{~min}$ and then for $30 \mathrm{sec}$ immediately prior to sampling. The liquid was drained into a fresh $125 \mathrm{~mL}$ separatory funnel while the Tenax resin remained on the sides of the old funnel. The resin was rinsed with $10 \mathrm{~mL}$ hexane. This rinse (resin and hexane) was collected in combusted glass $40 \mathrm{~mL}$ vials. The experiment was repeated three hours later with three new aliquots of the bacterial suspension (total $\mathrm{CB}$ incubation time $=5 \mathrm{~h}$ ). All samples were stored at $4^{\circ} \mathrm{C}$ until analysis.

\subsubsection{Ancillary measurements - bacterial concentrations and DOC.}

Population samples were preserved with $1 \%$ glutaraldehyde (v/v VSW). Bacterial cells were stained with acridine orange $(\mathrm{AO})$, drawn down onto black polycarbonate filters, and counted with epifluorescence microscopy (full method outlined in Chapter 2). Dissolved organic carbon samples were acidified with $50 \% \mathrm{H}_{3} \mathrm{PO}_{4}(100 \mu \mathrm{L}$ per $20 \mathrm{~mL})$ and measured with high-temperature combustion (method of Peltzer and Brewer (1993) analyzed at UMass-Boston in laboratory of R. Chen). Each sample was measured in triplicate. The DOC concentrations for incubation 1 and incubation 2 were $24.1 \pm 2.4$ $\mathrm{mgC} / \mathrm{L}$ and $20.5 \pm 0.5 \mathrm{mgC} / \mathrm{L}$, respectively.

\subsubsection{Sample work-up}

Congeners \#14 (3,5,-dichlorobiphenyl), \#103 (2,2',4,5',6-pentachlorbiphenyl), and \# 198 (2,2',3,3',4,5,5',6-octachlorobiphenyl) were used as surrogate standards. All compounds were purchased from AccuStandard (New Haven, CT) as solutions of $35 \mu \mathrm{g} / \mathrm{mL}$ in iso-octane. A "working" solution of all three of these compounds contained approximately $30 \mathrm{ng} / \mathrm{mL}$ each congener in acetone. Prior to analysis, $150 \mu \mathrm{L}$ of this 
working solution was added to each sample. The quantitation standard used was octachloronaphthalene (Supelco - Belafonte, PA: Cat\# 44-2757; Lot\# LA75076). A "working" solution of $60 \mathrm{pg} / \mu \mathrm{L}$ was made by diluting a stock solution of $60.3 \mu \mathrm{g} / \mathrm{mL}$ with iso-octane.

Aqueous samples were transferred to combusted $50 \mathrm{~mL}$ glass centrifuge tubes. The original sample vials were rinsed once with acetone and once with hexane. The combined rinses and samples were acidified to $\mathrm{pH} 2$ with hexane-rinsed $1 \mathrm{~N} \mathrm{HCl} \mathrm{(3-4}$ drops in $20 \mathrm{~mL}$ sample) and then extracted $5 \mathrm{X}$ with $5-6 \mathrm{~mL}$ hexane. Samples were mixed vigorously with a vortex mixer (Fisher Scientific) during each solvent extraction. The organic phase was removed with a Pasteur pipet and transferred to a round-bottom flask. Emulsions were rare and if present, were too small to accurately transfer. All extractions were combined in a round-bottom flask and dried with anhydrous $\mathrm{Na}_{2} \mathrm{SO}_{4}$. The hexane phase was then transferred to a fresh round-bottom flask and roto-evaporated until the solvent was reduced to $2-3 \mathrm{~mL}$. Solvent-exchange into hexane was facilitated by the addition of $15 \mathrm{~mL}$ hexane and subsequent roto-evaporation.

Hexane samples were dried with anhydrous $\mathrm{Na}_{2} \mathrm{SO}_{4}$ (Fisher Scientific combusted at $450^{\circ} \mathrm{C}$ for $4-5$ hours). The hexane phase and two subsequent rinses $\mathrm{Na}_{2} \mathrm{SO}_{4}$ were transferred to a solvent-rinsed round-bottom flask taking care to avoid any remaining Tenax resin. Each sample was roto-evaporated to a small volume (2-3mL). To ensure complete solvent-exchange into hexane, $15 \mathrm{~mL}$ hexane was added to the extract and the sample was roto-evaporated again.

All extracts were cleaned with concentrated $\mathrm{H}_{2} \mathrm{SO}_{4}$ after the method of Bergen et al. (1993). Extracts were transferred to $10 \mathrm{~mL}$ combusted screw cap centrifuge tubes. Concentrated $\mathrm{H}_{2} \mathrm{SO}_{4}$ was added to the extracts such that the volume ratio was 1:2 acid:solvent. The extracts were vortexed at medium speed (setting $=4$ ) for one minute. The tubes were then allowed to sit for $45 \mathrm{~min}$. The organic phase was then transferred with a Pasteur pipet to a combusted $4 \mathrm{~mL}$ glass vial. The acid phase was re-extracted twice with a small volume of hexane. All organic phases were combined in the combusted $4 \mathrm{~mL}$ vial. The organic phase was re-extracted with $300-500 \mu \mathrm{L}$ Milli-Q water 
once to remove any residual $\mathrm{H}_{2} \mathrm{SO}_{4}$. All extracts were then pipetted off the aqueous phase and reduced to near-dryness with ultra-high purity $\mathrm{N}_{2}$. Quantitation standard $(150 \mu \mathrm{L})$ was added to each extract prior to transfer with a Pasteur pipet to a $\mathrm{GC}$ vial with combusted $200 \mu \mathrm{L}$ insert. All samples were stored in the refrigerator until analysis.

\subsubsection{GC analysis and data generation}

All samples were analyzed on a gas chromatograph (HP 5890 Series II) with an electron capture detector (HP Model \#G1223A) and a 60m DB-5 capillary column (JT Baker). Analysis conditions consisted of the following temperature program: $60^{\circ} \mathrm{C}$ for $2 \mathrm{~min}$, ramp at $6^{\circ} \mathrm{C} / \mathrm{min}$ to $170^{\circ} \mathrm{C}$, ramp at $0.7^{\circ} \mathrm{C} / \mathrm{min}$ to $230^{\circ} \mathrm{C}$, hold for $15 \mathrm{~min}$, ramp at $2^{\circ} \mathrm{C} / \mathrm{min}$ to $298^{\circ} \mathrm{C}$ and hold for $5 \mathrm{~min}$ - with He as the carrier gas flowing at $1.2 \mathrm{~mL} / \mathrm{min}$. Five levels of a standard were analyzed at the beginning of each run. A standard was then run every six samples to monitor changes in column conditions. Chromatograms were integrated with HP ChemStation software.

Response factors for each of the surrogate standards relative to octachloronaphthalene $(\mathrm{OCN})$ and for each congener relative to each surrogate standard were calculated using all standards analyzed. Surrogate recoveries were calculated using areas for surrogate standards and $\mathrm{OCN}$ in each sample. These recoveries averaged $28.3 \pm 8.7 \%$ for $\# 14,67.3 \pm 13.8 \%$ for $\# 103$, and $57.7 \pm 13.0 \%$ for \#198 (range:\#14- 5.0\%55.4\%; \#103- 26.0\%-103.9\%; \#198- 26.6\%-85.4\%, $n=54$ ). Samples with $<40 \%$ recovery were removed from further analysis. The concentrations of each congener in an extract were calculated using the average response factor relative to one of the three surrogate standards. In general, the response factor relative to \#14 was used for congeners 8,18 , and 28 ; the response factor relative to \#103 was used for congeners $44,47,52,66,77$, $101,105,118,126,128,138,153$, and 169; and the response factor relative to \#198 was used for the remaining congeners.

Data from all 23 congeners could not be used due to analytical difficulties. The surrogate standard \#14 was usually too low to be considered a robust measurement and so the low-chlorine congeners, 8,18 , and 28 , were removed from further analyses. 
Congener \#47 often co-eluted with a phthalate contamination peak. The magnitude of this contamination peak varied unpredictably and could not be subtracted to give a reasonable estimate of the magnitude of \#47. Congener \#169 was always extremely low in comparison to the expected magnitude. The initial aqueous concentrations were similar to those expected from the amount added so problems with the initial \#169 spike could be ruled out. No satisfactory explanation for this behavior is known at this time. Congener \#206 was a split peak with OCN, the quantitation standard. In general, the recoveries of \#206 were thus over-estimated due to overlap with OCN. This could also not be corrected because the degree of overlap was a function of the mass of \#206 in the sample. This did not interfere significantly with the quantitation standard, $\mathrm{OCN}$, because the peak was much larger for OCN than \#206.

\subsection{Results}

The bacterial data are not considered in the remainder of this chapter. The loss of CBs from the bacterial size fraction is faster than the extraction rate into the Tenax resin (control experiment $\left(0.067 \pm 0.009 \mathrm{~min}^{-1}\right)$ with theoretical calculation from Chapter 2 $\left.\left(0.38 \mathrm{~min}^{-1}\right)\right)$. Therefore, this data cannot be used to determine the specific loss rate constant of $\mathrm{CBs}$ from bacteria. However, these data can be used to set a lower limit for $\mathrm{CB}$ loss from bacteria $\left(0.067 \mathrm{~min}^{-1}\right)$. To my knowledge, this is the first experimental evidence of full depuration of PCBs from bacteria and determination of a loss rate constant. In addition, interesting phenomena can be discussed in terms of the difference in extraction rate constants observed in the presence and absence of DOC.

\subsubsection{Calculation of expected and measured extraction rate constants}

The over-riding assumptions in the data analysis described below are that only truly dissolved PCBs are extracted by the Tenax resin and that the kinetics of this process are first-order (with respect to aqueous CB concentration). The first-order extraction rate constant is a function of the fraction of PCBs available for extraction, i.e., the fraction 
that is truly dissolved, $\mathrm{f}_{\mathrm{aq}}$. The rate equation used to describe this system can be written as:

$$
\text { (1) } \frac{d[C B]_{T x}}{d t}=k_{e x t r}^{p r e d t} *[C B]_{a q}=f_{c t q} * k_{c x t r}^{c o n t} *[C B]_{a q}
$$

where: $[\mathrm{CB}]_{\mathrm{Tx}}$ and $[\mathrm{CB}]_{\mathrm{aq}}$ are the $\mathrm{CB}$ concentrations in the Tenax resin and the aqueous phase, respectively $(\mathrm{pg} / \mathrm{mL}) ; \mathrm{k}^{\text {pred }}$ and $\mathrm{k}^{\mathrm{cont}}$ are the predicted and control extraction rate constants, respectively $\left(\mathrm{min}^{-1}\right)$; and $\mathrm{f}_{\mathrm{aq}}$ is the fraction of PCBs in the aqueous phase. The control extraction rate constant is the rate constant determined in the control experiments. This control extraction rate constant is multiplied by the fraction that can be extracted, i.e., the fraction of PCBs in the aqueous phase.

Wall losses in all experiments need to be considered by calculating $f_{\text {aq }}$. In the control experiments, wall losses will be more important because the majority of the aqueous PCBs are truly dissolved. Previous work with these systems indicated that approximately $43 \%$ of the total IUPAC \#77 was associated with the walls of the flask. Past studies were conducted with $50 \mathrm{~mL}$ of $0.3 \mu \mathrm{g} / \mathrm{mL}$ (total) solution in $125 \mathrm{~mL}$ Erlenmeyer flasks. The experiments in the present chapter were conducted with $100 \mathrm{~mL}$ solution in $125 \mathrm{~mL}$ separatory funnels. The increased surface area will most likely cancel the volume correction of the past data. Thus, I have assumed that the control experiments conducted with ${ }^{14} \mathrm{C}$-TCB in VSW lost $43 \%$ of the total to the walls of the separatory funnel. The control extraction rate constant, then, is the measured extraction rate constant $\left(0.033 \pm 0.005 \mathrm{~min}^{-1}\right)$ divided by the fraction aqueous $(0.57)$ and is equivalent to $0.067 \pm 0.009 \mathrm{~min}^{-1}$.

The calculation of $\mathrm{f}_{\mathrm{ak}}$ is central to predicting extraction rate constants for this experiment. The system described here contains four pools of PCBs - sorbed to bacteria, associated with DOC, associated with flask walls, and truly dissolved. The equation for calculating $f_{a q}$ is:

$$
\text { (2) } f_{t u}=\frac{1}{1+K_{D O C}[D O C]+K_{B}[B a c t] *[O C]_{b}+K_{w}[A r]_{w}}
$$


where $\mathrm{K}_{\mathrm{DOC}}$ and $\mathrm{K}_{\mathrm{B}}$ are the equilibrium partition coefficients for DOC/water and bacteria/water, respectively, $[\mathrm{DOC}]$ is the DOC concentration $\left(\mathrm{g} / \mathrm{mL}=10^{6} \mathrm{mg} / \mathrm{L}\right),[\mathrm{Bact}]$ is the concentration of bacteria $\left(3.73 \times 10^{7}\right.$ cells $\left./ \mathrm{mL}\right),[\mathrm{OC}]_{\mathrm{B}}$ is the organic carbon content per bacterial cell (70fg $\left(=10^{-15} \mathrm{~g}\right) / \mathrm{cell}$ - from Caron et al. $\left.(1991)\right) \mathrm{K}_{\mathrm{w}}$ is the wall-water partition coefficient and $[\mathrm{Ar}]_{\mathrm{w}}$ is the surface area of the walls in contact with solution.

The equilibrium partition coefficient for DOC/water $\left(K_{D O C}\right)$ was determined for congener $\# 77$ by headspace partitioning in Chapter 5 and is $10^{5.1}$. The equilibrium partition coefficient for bacteria/water $\left(\mathrm{K}_{\mathrm{B}}\right)$ was calculated using the partition coefficients for lipid/water $\left(\mathrm{K}_{\mathrm{lw}}\right)$ and for bacterial DOC/water $\left(\mathrm{K}_{\mathrm{DOC}}\right)$ as shown here:

(3) $K_{B}=f_{l i p} K_{l w}+\left(1-f_{l i p}\right) K_{D O C}$ where $f_{\text {lip }}$ is the fraction lipid in the bacterial cell (0.15 - ISwackhamer, $\left.\left.1993 \# 271\right]\right) . K_{\text {lw }}$ $\left(10^{6.33}\right)$ was calculated using the relationship in Swackhamer et al. (1993): $\log \mathrm{K}_{\mathrm{ww}}=$ $0.96^{*} \log \mathrm{K}_{\mathrm{ow}}+0.22$, where $\mathrm{K}_{\mathrm{ow}}\left(10^{6.36}\right)$ is the $n$-octanol/water partition coefficient from Hawker and Connell (1988). $\mathrm{K}_{\mathrm{DOC}}$ was used as the partition coefficient for the remainder of the bacterial cell because I assumed that the bacterial-derived DOC was similar in composition to the non-lipid cellular components within the bacterial cell. This assumption was derived from studies of bacterial-derived and grazer-enhanced DOC which showed that the DOC composition was similar to that of the bacterial cell (Taylor et al, 1985). Using these approximations, $K_{\mathrm{B}}$ was calculated to be $10^{5.63}$.

The product, $\mathrm{K}_{\mathrm{w}}{ }^{*}[\mathrm{Ar}]_{\mathrm{w}}$, was estimated from the following equation:

$$
\text { (4) } f_{c u f}=\frac{1}{1+K_{w}[A r]_{w}}
$$

which describes the aqueous fraction in a system with no DOC, analogous to the control experiment with ${ }^{14} \mathrm{C}$-TCB. This equation assumes equilibrium between the truly dissolved PCBs and the wall. From $\mathrm{f}_{a q}=0.57$, I calculated $\mathrm{K}_{\mathrm{w}}{ }^{*}[\mathrm{Ar}]_{\mathrm{w}}$ to be 0.769 . I then used this value in equation 2 for the calculation of $f_{a q}$ for both incubations. For incubation $1, \mathrm{f}_{\mathrm{aq}}$ was calculated to be 0.17 and for incubation $2, \mathrm{f}_{\mathrm{aq}}$ was calculated to be 0.19 . Given these calculations, I estimated that $\mathrm{k}^{\mathrm{pred}}$ should be $0.17^{*}(0.067 \pm 0.009)$ or $0.011 \pm 0.001$ $\min ^{-1}$ in incubation 1 and $0.19 *(0.067 \pm 0.009)$ or $0.013 \pm 0.002 \mathrm{~min}^{-1}$ in incubation 2 . 
The measured extraction rate constant, $\mathrm{k}^{\text {meas }}$, was calculated using the following equation:

$$
\text { (5) } k_{e x t r}^{\text {meas }}=\frac{\ln \left(\frac{T o t_{t}}{\text { Tot }_{0}}\right)}{t}
$$

where $\operatorname{Tot}_{\mathrm{t}}$ and $\mathrm{Tot}_{0}$ are the total CB concentrations present at time $t$ and 0 , respectively $(\mathrm{pg} / \mathrm{mL})$ and $t$ is time (min). When wall losses are taken into account, this equation becomes:

$$
\text { (6) } k_{e x t r}^{\text {mets }}=\frac{\ln \left(\frac{\operatorname{TotA} Q_{t}}{\operatorname{TotA} Q_{0}}\left(1-f_{w}\right)+2 f_{w}\right)}{t}
$$

where TotAQ and TotAQ $\mathrm{Q}_{0}$ are the total $\mathrm{CB}$ concentrations measured at time $t$ and 0 , respectively, and $f_{w}$ is the fraction associated with the wall. The wall fraction, $f_{w}$, was estimated from a previous experiment where wall losses in the presence of bacteria and DOC were measured. This formulation accounts for the fact that the measured Tenax extract (hexane rinse of Tenax resin) includes both the wall-associated and Tenaxassociated CB fractions. Total concentrations were used instead of aqueous concentrations because aqueous concentrations could not be accurately measured. This substitution is valid since the Tenax resin is extracting only the aqueous $\mathrm{CB}$ fraction Note: This assumption is not negated by the following discussion. See the discussion of thermodynamics versus kinetics at the end of the chapter]. For incubation 1, the average of the three trials was $0.059 \pm 0.023 \mathrm{~min}^{-1}$ and for incubation 2 , the average of the three trials was $0.051 \pm 0.009 \mathrm{~min}^{-1}$. These rate constants are a factor of $4-5$ greater than $\mathrm{k}^{\text {pred }}$, suggesting a significant enhancement for extraction rate in the presence of DOC.

\subsubsection{Estimate of $C B-D O C$ disassociation rate constant, $k_{\text {dis }}$}

The proposed DOC-enhanced diffusion can be compared to analogous situations in air-water transport. The example of formaldehyde is discussed extensively in Schwarzenbach et al. (1993) and is depicted in Figure 3-1. This analogy is instructive because it shows the apparent increased diffusive flux that can result from a chemical 
equilibrium reaction between a major and minor species (for the present study, the minor species is the truly dissolved congener and the major species is the CB-DOC complex). Formaldehyde exists in equilibrium with its diol counterpart ( $>99 \%$ exists as diol in aqueous solution). Both formaldehyde and its diol can diffuse to the air-water interface. However, only formaldehyde can move across this interface. This leaves an excess of the diol in the diffusive boundary layer which can dehydrate to form formaldehyde. The additional formaldehyde from the dehydration of the diol is then available for transport across the air-water interface. The formaldehyde-diol reaction and subsequent transport can be generalized for all reversible reactions.

When the reaction from $\mathrm{A}$ to $\mathrm{B}$ is represented by:

$$
A \stackrel{k_{1}}{\longrightarrow} B \text { and } A \stackrel{k_{2}}{\longleftarrow} B
$$

the increase in the flux of $A$ due to the back-reaction of $B$ is expressed by the following equation (from Schwarzenbach et al. (1993)):

$$
\text { (7) } \Psi=\frac{\text { Flux }(\text { reactive })}{\text { Flux }(\text { non }- \text { reactive })}=\frac{K_{e q}+1}{1+\left(\frac{K_{e q}}{q}\right) \tanh q}
$$

where $\Psi$ is the ratio between the flux of the reactive species and the flux of the nonreactive species, Flux(reactive) is the flux of the reactive species (i.e., compound that is augmented by reversible reaction, Flux (non-reactive) is the flux of the non-reactive species, $K_{e q}$ is the equilibrium constant of the A-B reaction $\left(k_{1} / k_{2}\right)$, and $q$ is a nondimensional parameter defined by:

$$
\text { (8) } q=\sqrt{\frac{k_{r}\left(z_{w}\right)^{2}}{D_{w}}}
$$

where $k_{r}$ is the sum of the forward and back reaction rate constants of the reversible reaction $\left(k_{1}+k_{2}\right)\left(s^{-1}\right), z_{w}$ is the width of the boundary layer $(m)$ and $D_{w}$ is the molecular aqueous diffusion coefficient of $\mathrm{A}\left(\mathrm{m}^{2} / \mathrm{s}\right)$.

This formulation can be applied to this system by treating the $\mathrm{CB} / \mathrm{DOC}$ interaction as a reversible reaction where $\mathrm{k}_{\mathrm{l}}$ is the reaction rate constant for the formation of the CB-DOC complex $\left(\mathrm{min}^{-1}\right)$ and $\mathrm{k}_{2}$ is the second-order disassociation rate constant 
$\left((\mathrm{g} \mathrm{OC})^{-1} \min ^{-1}\right)$. The "reactive" flux describes the case when aqueous PCBs in the diffusive boundary layer are augmented by the disassociation of the CB-DOC complex and the "non-reactive" flux occurs when the CB-DOC complex does not disassociate in the diffusive boundary layer. This formulation assumes that the aqueous molecular diffusion coefficients of the two species are equivalent. Molecular diffusion coefficients are dependent on molecular weight and thus CB-DOC complexes $(\mathrm{MW}=1000-10,000)$ are likely to have significantly lower diffusivities than the truly dissolved PCBs $(M W=200-400)$. Differences in the diffusivities of the complexes and their truly dissolved counterparts can range up to a factor of 7 (when $M W(D O C)=10,000$ ). For the congeners studied, this difference is offset by the large reservoir of PCBs present as CBDOC complexes.

The rate equation from Equation 1 can be expressed in terms of the flux into the Tenax resin as:

$$
\text { (9) } \frac{d[C B]_{T x}}{d t}=F_{l u x} * S A_{T x} *[T X]=k_{e x t r}^{\text {mets }}[C B]_{G{ }_{t}}
$$

where Flux $\mathrm{Tx}_{\mathrm{x}}$ is the flux into the Tenax resin $\left(\mathrm{g} \mathrm{m}^{-2} \mathrm{~s}^{-1}\right), \mathrm{SA}_{\mathrm{Tx}}$ is the surface area of the Tenax resin beads $\left(\mathrm{m}^{2} /\right.$ bead) and [TX] is the concentration of Tenax in the system (beads $/ \mathrm{mL}$ ). I can substitute $\mathrm{k}^{\text {meis }}$ and $\mathrm{k}^{\text {pred }}$ for reactive and non-reactive species, respectively, into equation 7 because both rate constants are divided by the same constant parameters, $\mathrm{SA}_{\mathrm{Tx}}$ and $[\mathrm{TX}] . \mathrm{K}_{\mathrm{eq}}$ in equation 7 is equivalent to $\mathrm{K}_{\mathrm{DOC}}$. The nondimensional parameter $q$ was determined by trial and error to be 5.1 in incubation 1 and 4.4 in incubation 2. For this system, $z_{\mathrm{w}}$ is assumed to $100 \mu \mathrm{m}$ (approximate diffusion boundary layer from Schwarzenbach et al. (1993). For congener $\# 77, D_{w}$ is $8 \times 10^{-6} \mathrm{~cm}^{2} / \mathrm{s}$ (from relationship in Schwarzenbach et al. (1993) calculated in Chapter 2). Using these values, $k_{r}$ was calculated to be $124.8 \mathrm{~min}^{-1}$ in incubation 1 and $92.9 \mathrm{~min}^{-1}$ in incubation 2 . Using the relationships $\mathrm{K}_{\mathrm{DOC}}=\mathrm{k}_{1} / \mathrm{k}_{2}, \mathrm{k}_{\mathrm{r}}=\mathrm{k}_{1}+\mathrm{k}_{2}$, and $\mathrm{k}_{\mathrm{dis}}=\mathrm{k}_{2} /[\mathrm{DOC}]$, I calculated $\mathrm{k}_{\mathrm{dis}}$ (the pseudo-first order disassociation rate constant) to be $42.5 \mathrm{~min}^{-1}$ in incubation 1 and $38.0 \mathrm{~min}^{-1}$ in incubation 2. This value of $\mathrm{k}_{\mathrm{dis}}$ is a lower-limit because I cannot take into account the adsorption of DOC onto the Tenax resin. 


\subsubsection{Extension of results to other congeners}

Extension of these results to the other congeners studied requires the estimation of $\mathrm{k}^{\text {pred }}$ for the other congeners. Extraction rate constants determined by Cornelissen $e t$ al. (1997) differed by $20 \%$ over 4 orders of magnitude in $\mathrm{K}_{\text {ow }}$. The rate-limiting step in the uptake of PCBs by Tenax resin is diffusion across the stagnant boundary layer as expressed in equation 9. This assumption has been made by others (e.g., Dulfer $e$ t al., 1996) and is appropriate here. If uptake onto the Tenax resin were the rate-limiting step in the extraction of PCBs, there would be no enhancement in the presence of DOC (unless the DOC is changing the surface of the resin). Enhancement of the extraction rate constant was observed in the presence of DOC for IUPAC \#77, suggesting that transfer across the stagnant boundary layer was the rate-limiting step in this system.

The extraction rate constant is a function of the aqueous molecular diffusion coefficient, $\mathrm{D}_{\mathrm{w}}$, the width of the boundary layer, $\mathrm{z}_{\mathrm{w}}$, the surface area of the resin, $S \mathrm{~A}_{\mathrm{TX}}$, and the concentration of Tenax [TX] as written below:

$$
k_{e x t r}^{\text {pred }}=\frac{F l u x_{w}}{S A_{T x} *[T X]^{*}[C B]_{d}}=\frac{D_{w}[C B]_{d}}{z_{w}{ }^{*} S A_{T x} *[T X]^{*}[C B]_{d}}=\text { const }^{*} D_{w}
$$

All parameters in the above equation are constant among the $\mathrm{CB}$ congeners except $\mathrm{D}_{\mathrm{w}}$. I determined the combination of constants (const) for congener \#77 and then used it to estimate $\mathrm{k}^{\text {pred }}$ for the remaining congeners. The appropriate $\mathrm{f}_{\mathrm{aq}}$ was then calculated for each congener using equation $2 . \mathrm{K}_{\mathrm{DOC}}$ was also extended for the remaining congeners. In Chapter $5, \mathrm{~K}_{\mathrm{DOC}}$ equals $0.95^{*} \mathrm{~K}_{\mathrm{oc}}$ where $\mathrm{K}_{\mathrm{oc}}$ is the organic carbon / water partition coefficient from Schwarzenbach et al. (1993). This correction was applied to the values of $\mathrm{K}_{\mathrm{oc}}$ for the other congeners studied. For each congener, values for $\mathrm{k}_{\mathrm{ex}}{ }^{\text {cont }}$ and $\mathrm{f}_{\mathrm{aq}}$ were calculated and used to estimate $\mathrm{k}^{\text {pred }}, \mathrm{k}^{\text {meas }}, \mathrm{k}_{\mathrm{r}}$ and $\mathrm{k}_{\mathrm{dis}}$ (Tables 3-2 and 3-3). The expected and measured rate constants, $\mathrm{k}^{\text {pred }}$ and $\mathrm{k}^{\text {meas }}$, were plotted against $\log \mathrm{K}_{\mathrm{ow}}$ in Figure 3-2. In both incubations, the difference (ratio) between $\mathrm{k}^{\text {pred }}$ and $\mathrm{k}^{\text {meas }}$ is almost constant across the suite of congeners. The independence of the ratio of $\mathrm{k}^{\text {pred }}$ to $\mathrm{k}^{\text {meas }}$ was surprising because a dependence on congener hydrophobicity was presumed. 


\begin{tabular}{|c|c|c|c|c|c|c|c|}
\hline Congener & $\begin{array}{l}\text { Log } \\
\text { Kow }_{\text {ow }}\end{array}$ & $\overline{F_{a q}}$ & $\begin{array}{c}\mathbf{k}_{\text {extr }} \\
\text { predicted }\end{array}$ & $\mathbf{k}_{\text {extr }}$ measured & ratio & $\begin{array}{c}\mathrm{k}_{\mathrm{r}} \\
\left(\mathrm{min}^{-1}\right)\end{array}$ & $\begin{array}{c}k_{\mathrm{dis}}{ }^{-1} \\
\left(\mathrm{~min}^{-1}\right)\end{array}$ \\
\hline 44 & 5.75 & 0.390 & 0.026 & $0.065(0.020)$ & 2.48 & 27.6 & 28.1 \\
\hline 52 & 5.84 & 0.361 & 0.024 & $0.060(0.021)$ & 2.49 & 27.6 & 23.9 \\
\hline 66 & 6.20 & 0.218 & 0.015 & $0.070(0.031)$ & 4.82 & 110.6 & 50.2 \\
\hline 77 & 6.36 & 0.172 & 0.011 & $0.059(0.023)$ & 5.14 & 124.8 & 42.5 \\
\hline 101 & 6.38 & 0.169 & 0.010 & $0.040(0.015)$ & 3.86 & 73.0 & 24.0 \\
\hline 105 & 6.65 & 0.112 & 0.007 & $0.032(0.016)$ & 4.63 & 101.6 & 20.6 \\
\hline 118 & 6.74 & 0.097 & 0.006 & $0.035(0.016)$ & 5.72 & 155.9 & 26.9 \\
\hline 126 & 6.89 & 0.078 & 0.005 & $0.027(0.016)$ & 5.62 & 150.5 & 19.8 \\
\hline 128 & 6.74 & 0.096 & 0.006 & $0.026(0.017)$ & 4.66 & 101.6 & 17.5 \\
\hline 138 & 6.83 & 0.082 & 0.005 & $0.022(0.013)$ & 4.54 & 97.2 & 14.2 \\
\hline 153 & 6.92 & 0.071 & 0.004 & $0.025(0.014)$ & 6.01 & 172.8 & 21.6 \\
\hline 170 & 7.27 & 0.041 & 0.002 & $0.013(0.011)$ & 5.82 & 161.5 & 10.7 \\
\hline 180 & 7.36 & 0.034 & 0.002 & $0.013(0.007)$ & 6.65 & 215.5 & 12.2 \\
\hline 187 & 7.17 & 0.046 & 0.002 & $0.015(0.012)$ & 6.08 & 178.6 & 14.2 \\
\hline 195 & 7.56 & 0.024 & 0.001 & $0.011(0.011)$ & 8.92 & 380.2 & 15.0 \\
\hline 199 & 7.20 & 0.042 & 0.002 & $0.012(0.010)$ & 5.47 & 145.2 & 11.0 \\
\hline 209 & 8.18 & 0.007 & 0.0004 & $0.018(0.013)$ & 50.4 & 12484.8 & 162.5 \\
\hline
\end{tabular}

Table 3-2. Parameters and extraction rate constants for incubation I (2h).

Column 1: $\log \mathrm{K}_{\mathrm{ow}}$ values from Hawker and Connell (1988); column 2: the fraction of the CB that exists in the aqueous phase, calculated using equation 2 ; column 3 : the predicted $k_{\text {extr }}$ or $k^{\text {pred }}$ using the fraction aqueous (equation 1); column 4: the measured $k_{\text {extr }}$ in incubation 1 , this is the average of the three trials \pm $1 \sigma$; column 5 : the ratio of the measured $\mathrm{k}_{\text {extr }}$ to the predicted $\mathrm{k}_{\text {extr }}$; column 6 : the resultant $\mathrm{k}_{\mathrm{r}}$ as calculated by equation 6 and column 7: the disassociation rate constant, $\mathrm{k}_{\mathrm{dis}}$, of the CB-DOC complex. [DOC] in incubation 1 was $24.1 \pm 2.4 \mathrm{mgC} / \mathrm{L}$.

\begin{tabular}{|c|c|c|c|c|c|c|c|}
\hline Congener & $\begin{array}{l}\text { Log } \\
\text { Kow }\end{array}$ & $\bar{F}_{\mathrm{aq}}$ & $\begin{array}{c}\mathbf{k}_{\text {extr }} \\
\text { predicted }\end{array}$ & $\mathrm{k}_{\mathrm{extr}}$ measured & ratio & $\begin{array}{c}k_{r} \\
\left(\min ^{-1}\right)\end{array}$ & $\begin{array}{c}k_{\text {dis }}{ }^{-1} \\
\left(\mathrm{~min}^{-1}\right)\end{array}$ \\
\hline 44 & 5.75 & 0.417 & 0.028 & $0.057(0.006)$ & 2.19 & 23.23 & 28.4 \\
\hline 52 & 5.84 & 0.388 & 0.026 & $0.050(0.004)$ & 2.08 & 19.2 & 19.9 \\
\hline 66 & 6.2 & 0.237 & 0.016 & $0.067(0.011)$ & 4.61 & 101.6 & 55.3 \\
\hline 77 & 6.36 & 0.188 & 0.013 & $0.051(0.009)$ & 4.43 & 92.9 & 38.0 \\
\hline 101 & 6.38 & 0.185 & 0.011 & $0.055(0.011)$ & 5.27 & 134.8 & 53.2 \\
\hline 105 & 6.65 & 0.123 & 0.008 & $0.046(0.011)$ & 6.67 & 215.5 & 52.4 \\
\hline 118 & 6.74 & 0.107 & 0.007 & $0.045(0.0 / 1)$ & 7.39 & 262.8 & 54.3 \\
\hline 126 & 6.89 & 0.086 & 0.005 & $0.029(0.008)$ & 6.00 & 172.8 & 27.3 \\
\hline 128 & 6.74 & 0.106 & 0.006 & $0.035(0.011)$ & 6.23 & 184.5 & 38.1 \\
\hline 138 & 6.83 & 0.090 & 0.005 & $0.027(0.008)$ & 5.7 & 155.9 & 27.4 \\
\hline 153 & 6.92 & 0.078 & 0.004 & $0.033(0.008)$ & 8.04 & 307.2 & 46.0 \\
\hline 170 & 7.27 & 0.045 & 0.002 & $0.026(0.016)$ & 11.8 & 668.3 & 53.4 \\
\hline 180 & 7.36 & 0.038 & 0.002 & $0.024(0.013)$ & 12.5 & 750 & 51.0 \\
\hline 187 & 7.17 & 0.051 & 0.003 & $0.030(0.018)$ & 11.9 & 691.2 & 66.1 \\
\hline 195 & 7.56 & 0.027 & 0.001 & $0.021(0.016)$ & 16.4 & 1291.0 & 61.3 \\
\hline 199 & 7.2 & 0.046 & 0.002 & $0.023(0.017)$ & 10.4 & 529.2 & 47.9 \\
\hline 209 & 8.18 & 0.008 & 0.0004 & $0.012(0.012)$ & 34.4 & 5680.1 & 88.7 \\
\hline
\end{tabular}

Table 3-3. Parameters and extraction rate constants for incubation 2 (5h).

Data prepared in same manner as Table 3-2. [DOC] in incubation 2 was $20.5 \pm 0.5 \mathrm{mgC} / \mathrm{L}$. 


\subsection{Discussion}

\subsubsection{Implications for protozoan uptake of PCBs and microbial food web}

One of the concerns in the previous chapter was the role of DOC in the transfer of PCBs from the aqueous phase into protozoa in an experimental system. CB-DOC * complexes will be involved in both ingested and diffusive uptake of PCBs by the protozoa. First, in cultures with high DOC concentrations, protozoa are capable of incorporating dissolved material through pinocytosis, or the invagination of the cellular membrane around a parcel of water. In addition, during the process of phagocytosis, protists will be ingesting CB-DOC complexes along with contaminated bacteria. This data set has shown that $\mathrm{CB}-\mathrm{DOC}$ complexes can also enhance diffusive transport to the cellular membrane prior to adsorption to the cellular surface. The question then is whether CB-DOC complexes can tip the balance between diffusive and ingestive uptake such that ingestion out-competes diffusion for the primary mode of $\mathrm{CB}$ uptake.

Imagine a system such as that in this bacterial experiment where $20 \%$ of the PCBs are in the aqueous phase, $20 \%$ are in the bacterial pool and the remaining $60 \%$ are associated with DOC and assume the protozoa incorporate the bacteria and DOC and retain all the PCBs that are within these phases. This would increase the PCBs the protozoa can access through ingestion from $20 \%$ to $80 \%$. Therefore, ingestion would increase by a factor of 4 . Diffusion would also increase - from $20 \%$ to $60-70 \%$. This translates into an increase of a factor of about 3 for diffusion. From the theoretical calculation and the experiments in the previous chapter, we suppose that diffusion is faster than ingestion by a factor of 10000 . The small increase in ingestion by the incorporation of CB-DOC complexes is not enough to make ingestion the primary mode of $\mathrm{CB}$ uptake. This implies that even in areas of high DOC concentration (such as porewaters and sediment-water interface micro-environments), diffusion will be more important than ingestion for protozoan $\mathrm{CB}$ uptake. 


\subsubsection{Implications for the concept of "bioavailability"}

The term "bioavailability" has been used in many settings with different inherent meanings. In many studies, bioavailability is defined as the fraction of a contaminant that can be accumulated by an organism under a particular set of conditions. This is different from the term, "exposure", which is defined as the fraction of a contaminant that can be accumulated by an organism over a certain period of time (under a particular set of conditions) (Hamerlink et al., 1994). At infinite time, exposure equals bioavailability. In relatively static environments where organisms can equilibrate with their surroundings over appropriate time scales, the difference between these two terms is easily observed. "Bioavailability" in this case refers to the thermodynamically predicted equilibrium concentration of organic contaminant within the organism. "Exposure" refers to the kinetic uptake of a contaminant within an organism. In environments that are subject to pulses of high contaminant influx, the difference between these two terms is more difficult to observe. In such dynamic systems, there may not be enough time to allow full equilibration of all organisms. Therefore the "bioavailable" fraction is a function of time and so approaches the definition of "exposure". Many studies do not specify which term they are measuring and use the term "bioavailability" indiscriminately. [Note: For a complete discussion of this topic, the reader is refered to Hamerlink et al. (1994)].

For the purposes of this discussion, these two terms will be used as defined for a static system. "Bioavailability" in this definition will be a function of the aqueous contaminant concentration, according to the partition coefficient, $K_{\mathrm{p}}$. With the aqueous concentration and the equilibrium partition coefficient for a particular phase, one can predict the thermodynamic equilibrium concentration of PCBs in that phase, or the "bioavailability" to that phase. Given the rate constant of aqueous CB uptake, the time needed to achieve the thermodynamic equilibrium concentration can be estimated. The data in this chapter and its interpretation affect the estimate of the time needed to achieve equilibrium among the different pools and not the equilibrium concentration.

A number of studies have shown that certain kinetic barriers might exist that inhibit full equilibration of organisms with aqueous PCBs (exposure $\neq$ bioavailability). 
For example, the addition of biomass through growth diluted the PCBs in the organism because the rate of biomass addition was faster than the rate of $\mathrm{CB}$ uptake via diffusion (Swackhamer and Skoglund, 1993; Skoglund et al, 1996). The study presented in this chapter suggests a way to offset this growth issue and other kinetic barriers in high DOC environments. The enhancement of diffusion by CB-DOC complexes could lower the kinetic barrier(s) such that the effects of growth are not observed until organisms become large enough that surface area to volume ratios increase above the threshold where diffusive transport dominates.

The effect of CB-DOC complexes was present for all congeners studied within the uncertainties inherent in the estimations used. The magnitude of the enhancement (as expressed by the ratio of measured to expected extraction rate constant) was similar across the suite of congeners, regardless of hydrophobicity. The most likely explanation for this phenomenon is that the decrease in diffusivity and $f_{a q}$ were offset by the increased CB-DOC concentration.

DOC concentrations in this study were quite high $(20-24 \mathrm{mg} / \mathrm{L})$. DOC concentrations of this magnitude have been observed in past cultures with this bacterium (H.halodurans) (Barbeau, 1998). In the marine environment DOC concentrations are much lower (as low as $\leq 10 \%$ of this value). The effect of DOC-enhanced diffusion is not expected to be linearly dependent on DOC concentration because it affects only the fraction available for "extraction" or $f_{\text {aq }}$. The expected extraction rate constant is a function of $f_{\mathrm{aq}}$ and thus with decreased DOC concentrations, one would expect higher extraction rate constants.

The predicted effect of DOC concentration on the measured extraction rate constant is shown for three congeners in Figure 3-3. In the low (tetra-) chlorinated congener (IUPAC 77 - Figure 3-3A), the full effect of the DOC-enhanced diffusion will not be observed at DOC concentrations up to $30 \mathrm{mg} / \mathrm{L}$ because the predicted extraction rate constant is greater than the control (or maximum) extraction rate constant. For the mid- (hexa-) chlorinated biphenyl (IUPAC \#153 - Figure 3-3B), the ratio ( $\Psi$ ) between $\mathrm{k}^{\mathrm{pred}}$ and $\mathrm{k}^{\text {meas }}$ is higher than for $\# 77$ and the effect of DOC-enhanced diffusion is evident. 
Again no change from the maximum extraction rate constant will be observed until high DOC concentration $(17 \mathrm{mg} / \mathrm{L})$. After $\mathrm{DOC}=17 \mathrm{mg} / \mathrm{L}$, the observed extraction rate constant decreases but remains significantly above the expected extraction rate constant. Similar results were observed with a high- (octa-) chlorinated biphenyl (IUPAC \#195 - Figure 3$3 \mathrm{C})$. The observed extraction rate constant is predicted to decrease below the maximum extraction rate constant at $\mathrm{DOC}=10 \mathrm{mg} / \mathrm{L}$. For all congeners, then, DOC-enhanced diffusion is important. This suggests that this phenomenon should be considered in many aquatic systems along with congener hydrophobicity and may affect the assessment of "exposure" for organisms.

Semi-permeable membrane devices (SPMDs) have been used extensively to assess the "bioavailability" of contaminants in aqueous systems such as estuaries and aquifers (Huckins et al., 1993; Lebo et al., 1995; Hofelt and Shea, 1997; Sabaliunas et $a l ., 1998)$. These devices are composed of polyethylene tubing filled with a hydrophobic solvent or gel that adsorbs aqueous organic species that cross the tubing membrane (Huckins et al., 1993). In such a system, there are two candidates for the rate-limiting step of absorption. First, there is the diffusive boundary layer at the surface of the tubing. In this case, the CB-DOC complex will increase the diffusive uptake of PCBs by SPMDs within a period of time. SPMDs can be assumed to be indicators of organism "exposure" in such a situation. In the second case, the rate-limiting step is diffusion across the polyethylene membrane. The surface of the tubing is equilibrated with the aqueous PCBs and CB-DOC complexes have no effect on the accumulation of PCBs within the SPMDs. In this case, SPMDs can be assumed to be indicators of "bioavailability" in such an environment.

\subsection{Conclusions}

The study presented in this chapter had two important contributions. First, a lower-limit for the bacterial loss rate constant for PCBs was experimentally measured. Second, DOC-enhanced diffusion was observed for 13 congeners spanning the range of hydrophobicities present in the chlorobiphenyl compound class. The measured extraction 
rate constants of IUPAC \#77 into Tenax resin were significantly greater than those predicted from control experiments and estimates of the fraction available for extraction $\left(f_{a q}\right)$. This model assumes full equilibration with DOC in aqueous solution. The increase in extraction rate constant was attributed to diffusion of CB-DOC complexes into the diffusive boundary layer surrounding the Tenax beads and subsequent disassociation of the complexes. Using a relationship between the increase in extraction rate constant, a lower limit for the disassociation rate constant of the CB-DOC complex (IUPAC \#77) was estimated to be $38-42.5 \mathrm{~min}^{-1}$. These results were extended to other congeners studied. Significant enhancements were estimated for all congeners studied. This effect was predicted to be important in the diffusive uptake processes in systems with DOC concentrations up to $30 \mathrm{mg} / \mathrm{L}$. 


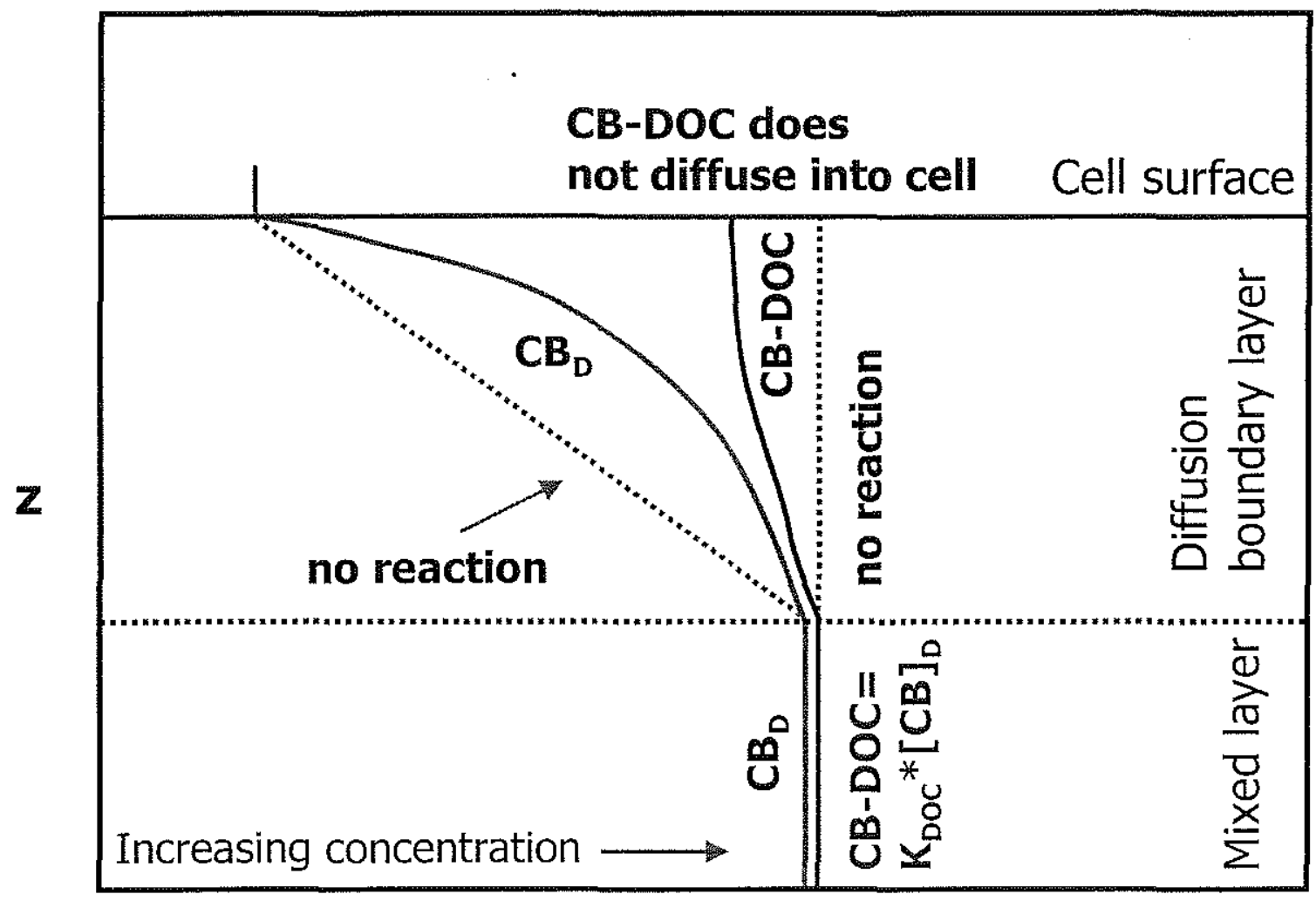

\section{Adapted from Schwarzenbach et al. 1993}

Figure 3-1. Schematic detailing enhanced diffusion due to $\mathrm{CB} / \mathrm{DOC}$ interactions. In the bulk solution or mixed layer, the dissolved $\mathrm{PCBs}\left(\mathrm{CB}_{\mathrm{D}}\right)$ and the $\mathrm{CB}-\mathrm{DOC}$ complexes are in equilibrium and their concentrations are constant and fixed by the DOC-water partition coefficient, $\mathrm{K}_{\mathrm{DOC}}$. At the cellular surface, the dissolved $\mathrm{CB}$ congener can diffuse into the cellular membrane but the CB-DOC complex cannot. In the absence of appreciable reaction between the dissolved $\mathrm{CB}$ and $\mathrm{CB}-\mathrm{DOC}$ complex, the concentrations of both species are represented by the dotted lines. The dissolved CB concentration decreases linearly toward the cell surface and this decrease is dependent on the aqueous molecular diffusion coefficient. The CB-DOC complex concentration is constant and equivalent to that in the bulk solution. However, when the $\mathrm{CB}-\mathrm{DOC}$ complex can disassociate on the time scale of dissolved CB diffusion to the cell surface, the concentrations of both species are represented by the solid lines. The concentration of CBDOC complex decreases as it disassociates to dissolved $C B$ and DOC. The disassociation increases the concentration of the dissolved $\mathrm{CB}$ congener. Since the uptake flux is determined by the slope of the concentration gradient at the cell surface, the increase in dissolved CB concentration leads to a steeper slope at the cell surface and thus an increase in diffusive flux into the cell. 

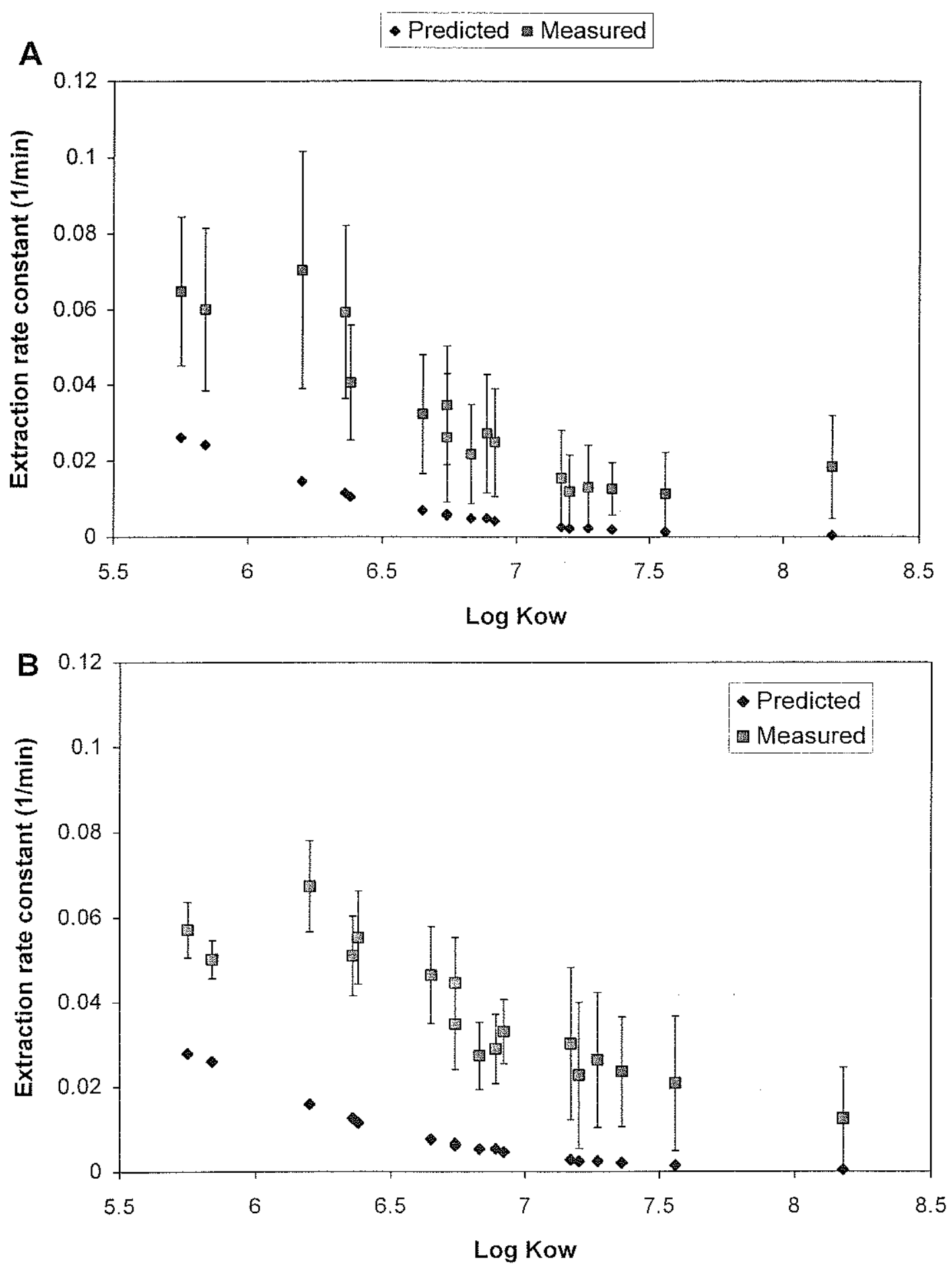

Figure 3-2. Expected and measured extraction rate constants versus $\log \mathrm{K}_{\mathrm{ow}}$ for both incubations. Expected extraction rate constants were calculated according to formulas presented in the text, modified from Schwarzenbach et al. (1993). Measured extraction rate constants are the average $\pm 1 \sigma$ of triplicate experiments run at two different $\mathrm{CB}$ incubation times (top figure $\mathbf{A}$ represents incubation $1(2 \mathrm{~h})$ and the bottom figure $\mathbf{B}$ represents incubation $2(5 \mathrm{~h})$ ). 


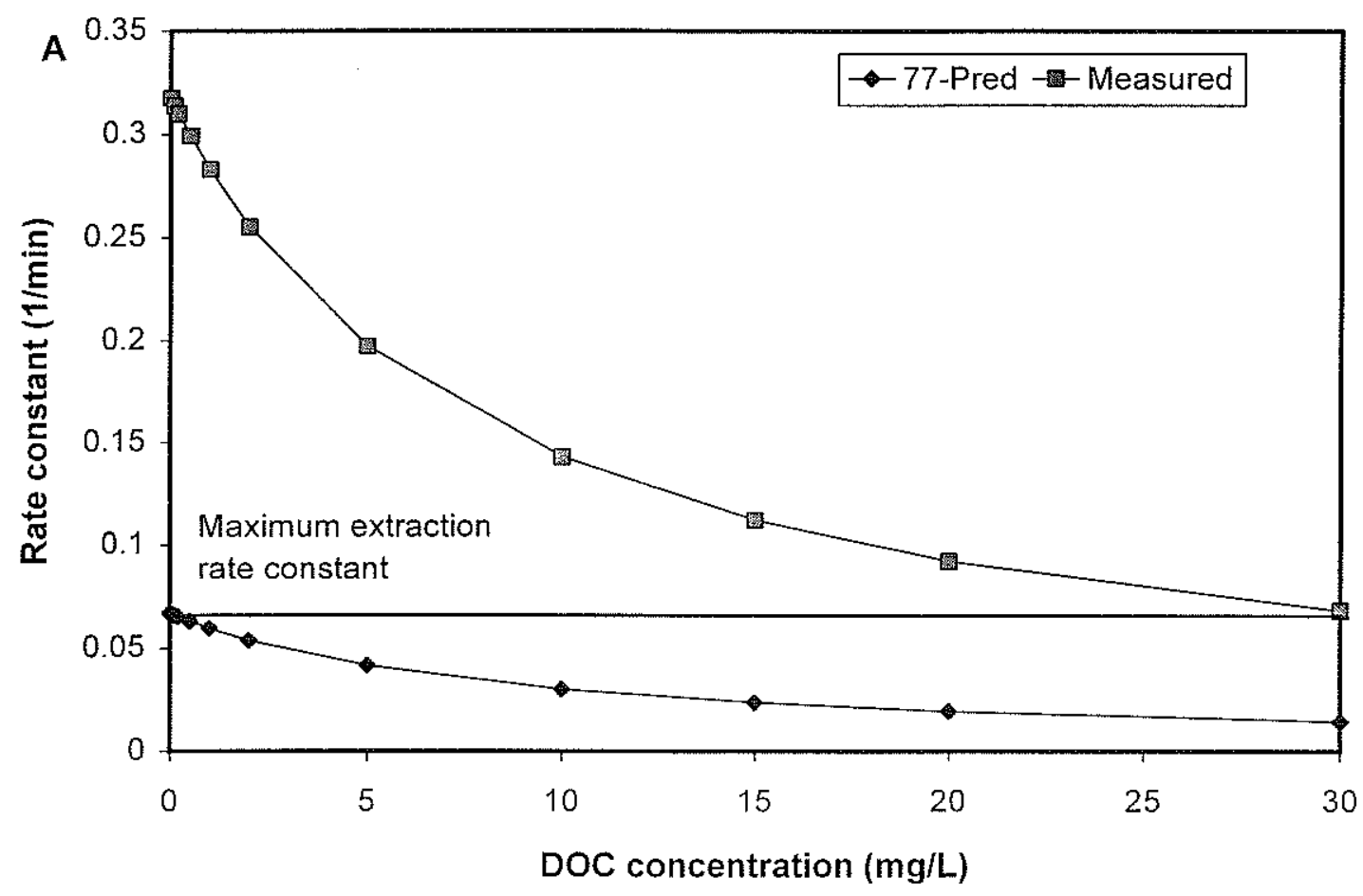

B $\rightarrow$ 153-exp- -153-meas

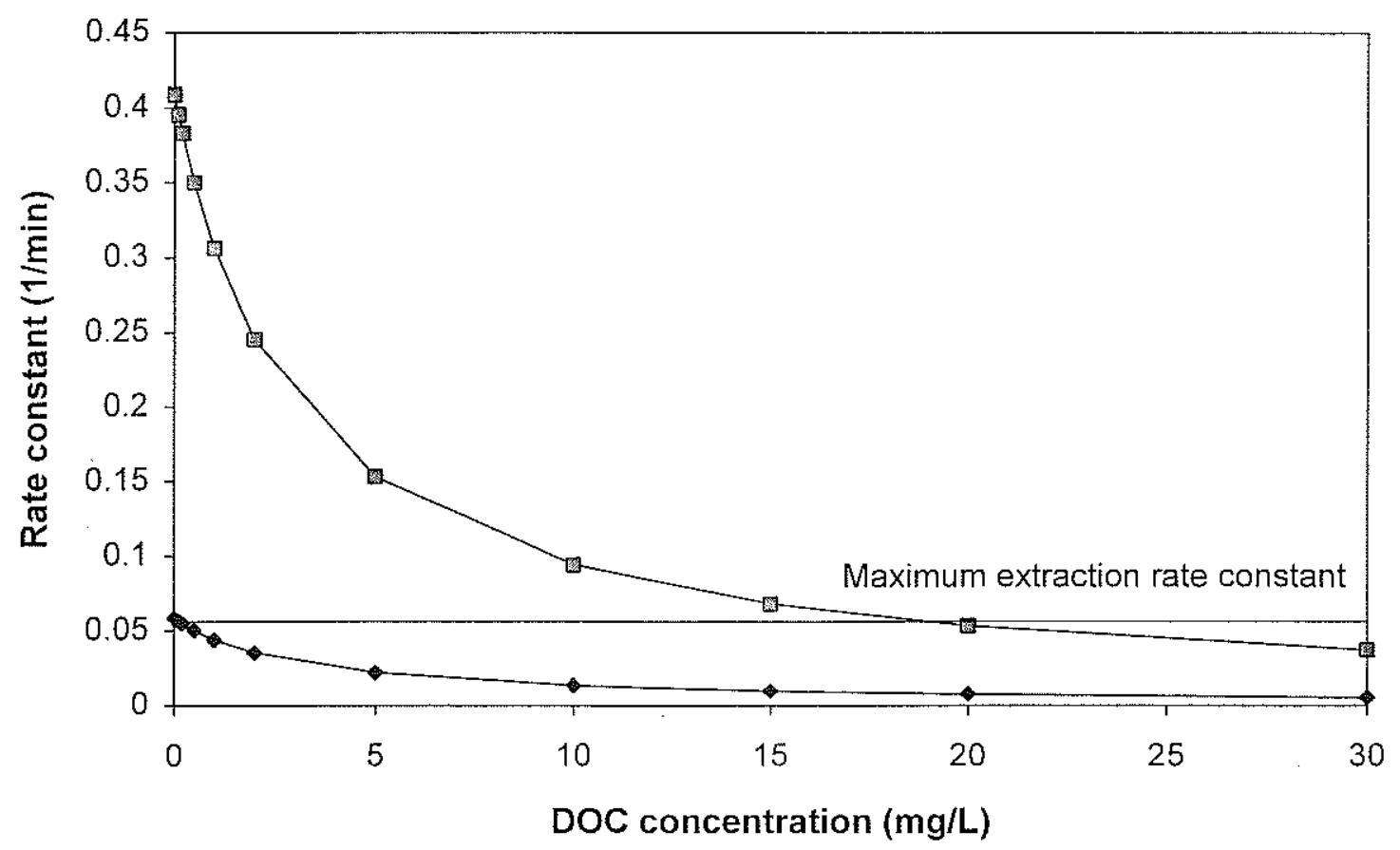




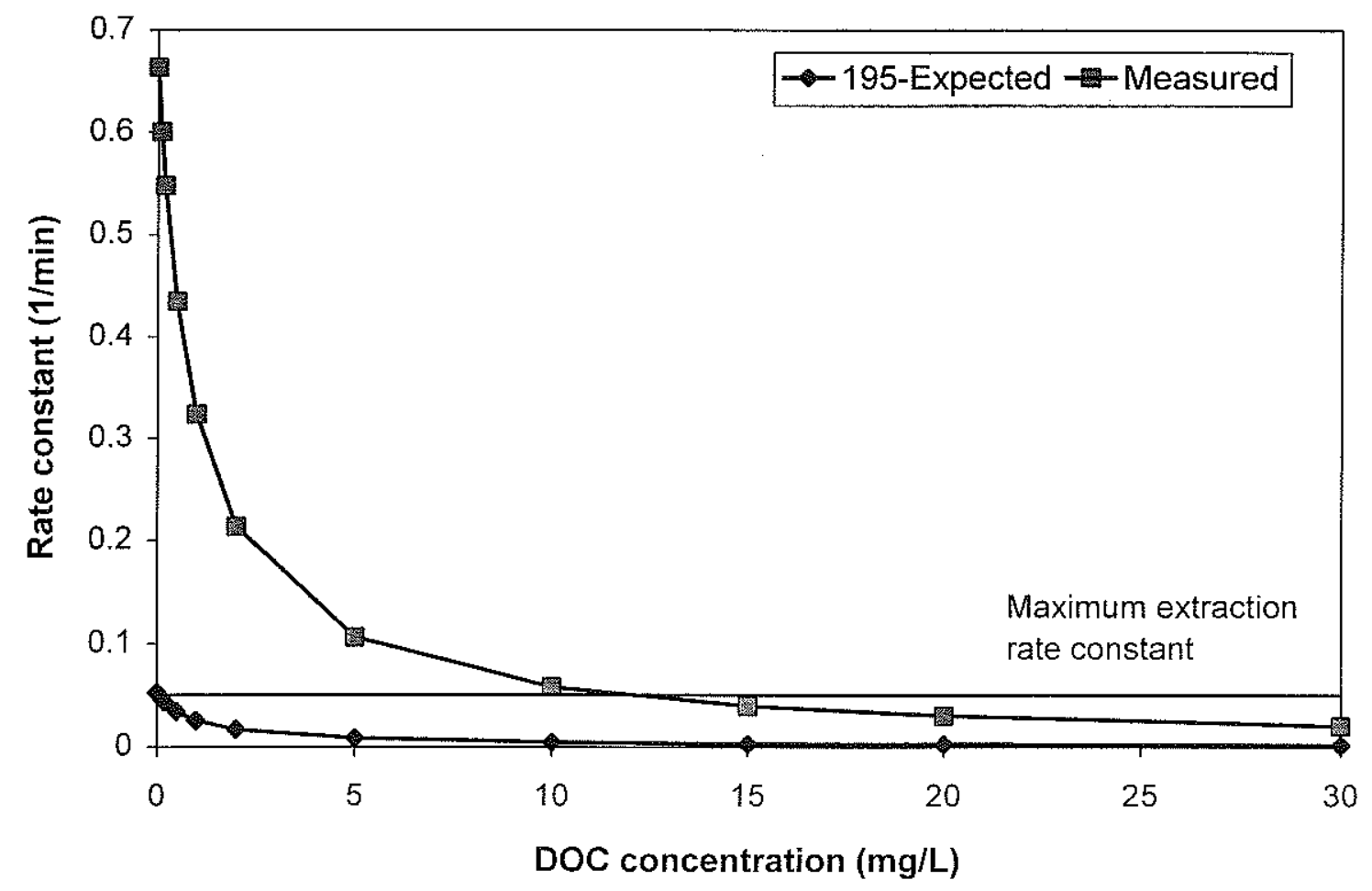

Figure 3-3. Expected and measured rate constants (predicted) for $3 \mathrm{CB}$ congeners as a function of DOC. The expected rate constants were calculated as $\mathrm{f}_{\mathrm{nq}}{ }^{*} \mathrm{k}^{\mathrm{cout}}$ as in Section 3.3.1. The "measured" rate constants were calculated by multiplying the predicted rate constant by $\Psi$, the ratio between reactive and nonreactive fluxes. The parameter $\Psi$ was calculated using the average $q$ value from Tables $3-1$ and $3-2$ for the appropriate $\mathrm{CB}$ congeners and the $\mathrm{K}_{\mathrm{DOC}}$ values from Chapter 5. A: IUPAC \#77 B: IUPAC \#153 C: IUPAC \#195. 


\section{Dissolved organic matter cycling in protozoan grazing cultures: Temporal and compositional dynamics}

\subsection{Introduction}

The dynamics and composition of dissolved organic matter have been extensively studied by numerous investigators (Lee and Wakeham, 1988; Mopper et al., 1991; Benner et al., 1992; Druffel et al., 1992; Carlson et al., 1994; Aluwihare et al., 1997; McCarthy et al., 1997). This pool of organic carbon represents the largest reservoir of reduced carbon in the ocean and is integral to the global carbon cycle. A number of processes govern both the concentration and the composition of this material (overview in Figure 4-1). Inorganic carbon enters the organic pool via photosynthetic fixation by photoautotrophs in the surface ocean. Through cell lysis, leakage, or senescence, a fraction of this fixed carbon enters the dissolved pool where it is utilized by bacteria (secondary producers) as an energy source. Grazing by micro- and nano-zooplankton packages some of the organic carbon into sinking aggregates. Other consequences of grazing and secondary production include respiration of $\mathrm{CO}_{2}$ and excretion / release of more dissolved organic matter and inorganic nutrients.

The ratio of net to gross primary production in different oceanic regimes is a measure of the strength of the recycling processes in those ecosystems. In oligotrophic areas, $10 \%$ or less of primary production is removed from the surface. This indicates that most material is cycled through a complex trophic system such as the microbial food web. To a first approximation, phytoplankton-derived organic matter is produced with C:N ratios similar to those observed by Redfield (1958). However, bulk DOM has been shown to have higher $\mathrm{C}: \mathrm{N}$ ratios than those proposed by Redfield, suggesting that labile, $\mathrm{N}$-rich compounds have been utilized more readily than $\mathrm{N}$-poor compounds. This observation implies that different components of DOM are affected to varying extents by degradation processes within the microbial food web. Cognizance of the effects of microbial cycling on different components of DOC is important because sub-pools of DOC play different roles in ocean processes. For example, surface-active material 
influences air-sea gas exchange (Frew et al., 1990) and lipid-rich material affects organic contaminant speciation. This chapter focuses on the effect of protozoan grazing processes on the production of surface-active material, lipid-rich DOM and bulk DOC within a simple microbial food web (predator + prey). While these results cannot be simply extended to natural systems, they are indicators of larger processes occurring in the surface ocean or at the sediment-water interface and can thus guide further experimentation into DOM cycling.

\subsubsection{Surfactants}

Surface-active material has a number of roles in the ocean. This material can be concentrated at the air-sea boundary and form a microlayer that affects air-sea gas exchange (Frew et al., 1990). Surface-active materials on particles can affect dissolvedparticulate metal dynamics (Shine and Wallace, 1995). The stickiness of surface-active material can also enhance aggregation of small colloids and particles and increase the flux of material out of the surface ocean (Mopper et al., 1995; Zhou et al., 1998). Marine surface-active material (surfactants) is presumed to be derived primarily from phytoplankton exudates and their degradation products (Zutic et al., 1981). Large concentrations of surfactant material have been observed during phytoplankton blooms (Sakugawa and Handa, 1985). Production of this material is seasonal and has been linked to biological productivity cycles (Cosovic et al., 1985).

Surface-active material contains both hydrophobic and hydrophilic components and has been shown to have a variety of structures. Organic matter of recent biological origin often has surface-active properties due to the presence of carboxylic acids as well as aliphatic carbon chains. Compositional studies of natural surfactants have shown the presence of carbohydrates, lipids and proteins, with a heavy dominance of polysaccharide compounds (Passow et al., 1994; Vojvodic and Cosovic, 1996). However, as noted by Frew et al. (1990), proteins and lipids containing both hydrophobic and hydrophilic moieties can play a role in the surface microlayer that is disproportionate to their relative concentration. 


\subsubsection{Microbial loop}

The microbial food web, or "microbial loop", is an assemblage of organisms that has been implicated in remineralization of organic material both at the sediment-water interface and in the water column (Pomeroy, 1974; Azam et al., 1983; Sherr and Sherr, 1988; Sherr and Sherr, 1994). This loop consists of bacteria, phytoplankton, and nanoand micro-zooplankton (Figure 4-2). The concept of a "loop" is especially appropriate since organic matter is constantly shuttled between and among different pools making up this micro-ecosystem. Key organisms in this system are the small protozoa which graze on both bacteria and small phytoplankton. Their waste material contains both organic matter (colloidal and dissolved) and inorganic nutrients which are then utilized by bacteria and picoplankton (Scavia, 1988). As mentioned above, much of the organic carbon originally reduced by photosynthesis is recycled and remineralized within this loop in oligotrophic systems. Within the microbial loop, protozoan grazers play an extremely important role in the determination of the concentration and composition of DOM.

Numerous studies have addressed the role of protozoan grazers in DOM cycling by attempting to discern the controlling factors in DOM release and composition by different species of protozoa. Prey type and growth stage were shown to affect DOM release by flagellates (Nagata and Kirchman, 1990; Nagata and Kirchman, 1992a). Other factors have included food selectivity (Caron et al., 1991), ingestion rate and assimilation efficiency (Jumars et al., 1989). In general, many studies have observed that DOM release was highest during exponential growth and very low during stationary growth (review - Nagata and Kirchman, 1992a). This phenomenon has been modeled by Jumars et al. (1989). They proposed that organisms acted to maximize food acquisition rather than digestive efficiency. High digestive efficiencies would be effective only in times of prey scarcity. When prey concentrations were high, ingestion rates would also be high. However, to maintain high ingestion rates, organisms would have short digestion cycles which result in low digestive efficiencies. Low digestive efficiency, or incomplete 
digestion of ingested prey, necessitated high DOM release by grazers, especially during blooms of prey (Jumars et al., 1989).

Studies of DOM release have begun to elucidate the composition of this material (Taylor et al., 1985; Nagata and Kirchman, 1992b; Tranvik, 1994). Taylor et al. (1985) stressed that DOC produced in grazing cultures was best described as "grazer-enhanced" release rather than "grazer-produced". Without direct evidence for biological synthesis of DOM, grazers were presumed to be causing release of DOM via sloppy feeding or incomplete digestion (Taylor et al., 1985). The first characteristic of this material to be addressed was its size. Pelegri et al. (1999) observed an increase in total organic matter in the $<1 \mu \mathrm{m}$ size fraction. They proposed that this material was a direct consequence of grazing by the nanoflagellate studied because they used a bacterial prey that was not metabolically active. Likewise, Taylor et al. (1985) observed that grazer-enhanced DOM was enriched in compounds with a nominal molecular weight of $10^{3}-10^{4}$ daltons. They also noted the release of macromolecular material $\left(>10^{5}\right.$ daltons) but this release was not significantly greater than in the bacterial control. Nagata and Kirchman (1992b), however, observed a significant release of macromolecular material (defined as the precipitate of a cold trichloroacetic acid extraction). Tranvik (1994) also noted the appearance of colloidal material $(0.02 \mu \mathrm{m}-0.2 \mu \mathrm{m})$ in flagellate grazing cultures. These three studies are not necessarily contradictory given the inherent variability in protozoan species and grazing conditions among these experiments. The common result of these three studies is the observed release of macromolecular/colloidal material within the larger pool of DOM égested by protozoan grazers.

Two of these studies also examined the composition of the released DOM using bacteria grown on radio-labeled substrates $\left({ }^{14} \mathrm{C}\right.$-glucose and ${ }^{14} \mathrm{C}$-leucine - (Tranvik, 1994) and ${ }^{3} \mathrm{H}$-glucose - (Nagata and Kirchman, 1992b)). In Tranvik's study, the two substrates labeled different cell components. The radio-label occurred primarily in the cell wall in bacteria grown on glucose and in internal cell components (presumably proteins or amino-sugars) in bacteria grown on leucine. Tranvik observed that colloidal material was radio-labeled in grazing cultures with leucine-grown prey. He therefore concluded that 
"grazer-enhanced" colloidal material $(0.02 \mu \mathrm{m}-0.2 \mu \mathrm{m})$ had a higher fraction of internal cell components than cell wall (Tranvik, 1994). He argued that protozoan digestive enzymes would be strong enough to solubilize a large fraction of the cell wall and force those compounds into the truly dissolved fraction $(<0.02 \mu \mathrm{m})$.

Nagata and Kirchman (1992b), on the other hand, concluded that colloids present in protozoan cultures were phospholipid micelles surrounding digestive enzymes. The phospholipids in the micelles would be derived from outer cellular membranes. These micelles were formed in the super-saturated protozoan food vacuole and trapped protozoan digestive enzymes within their centers. This hypothesis was based on different digestive enzyme activities depending on the presence or absence of phospholipases. In addition, the lipid composition of the "grazer-enhanced" DOM was assumed to be similar to bacterial cellular material because the lipid/macromolecular material ratio was similar in both cases. There are two caveats that should be considered when contemplating the Nagata and Kirchman (1992b) data set. First, the use of the lipid/macromolecular ratio to infer lipid-rich colloidal material assumed that the two extraction methods remove the same pool of material. Second, they proposed that phospholipid micelles formed in the vacuoles because their concentration in this microenvironment was above the critical micelle concentration. If this is the case, it is unclear why these micelles remained intact in the aqueous culture where the phospholipid concentration was significantly lower than the critical micelle concentration.

The third characteristic of "grazer-enhanced" DOM that was addressed in a number of studies was the lability of this material to bacterial uptake and degradation. In general, egested material is partially digested prey. As such, it should be more labile than whole prey cells. Over all, Jumars et al. (1989) predicted that the grazing process should increase the lability of bulk organic matter. While Tranvik (1994) noted that DOM from grazing experiments was more recalcitrant than phytoplankton-derived material, Taylor $e t$ al. (1985) observed that the bulk of DOM produced during grazing was available for bacterial utilization. They noted further that the size spectrum of the material shifted towards lower molecular weight compounds (Taylor et al., 1985). These studies used a 
myriad of protozoan species and prey. The species used in each experiment discussed above and the characteristics of the DOM produced are summarized in Table 4-1. In a natural assemblage of protists, all these characteristics will likely be present in "grazerenhanced" DOM.

\begin{tabular}{|c|c|c|c|}
\hline Protozoan species & Prey species & $\begin{array}{l}\text { Characteristics of } \\
\text { C/DOM produced }\end{array}$ & Reference \\
\hline $\begin{array}{l}\text { Euplotes, sp. } \\
\text { Uronema, sp. } \\
\text { (ciliates) }\end{array}$ & Mixed bacterial assemblage & $\begin{array}{c}500 \mathrm{NMW} \text { and } \\
10^{3}-10^{4} \mathrm{NMW} \\
\text { Readily utilized by } \\
\text { bacteria }\end{array}$ & Taylor et al. (1985) \\
\hline $\begin{array}{l}\text { Paraphysomonas } \\
\text { imperforata } \\
\text { (nanoflagellate) }\end{array}$ & $\begin{array}{l}\text { Mixture of bacteria: Vibrio } \\
\text { splendidus, } V . \text { damsela, } V . \\
\text { gazogenes, } V \text {. natriegens, } \\
V . \text { proteolyticus }\end{array}$ & $\begin{array}{c}<0.2 \mu \mathrm{m} \\
\text { C/DOM = Phospholipid } \\
\text { micelles surrounding } \\
\text { protozoan digestive } \\
\text { enzymes }\end{array}$ & $\begin{array}{c}\text { Nagata \& } \\
\text { Kirchman }(1992 b)\end{array}$ \\
\hline $\begin{array}{c}\text { Poterioochromonas } \\
\text { malhamensis } \\
\text { (mixotrophic flagellate) }\end{array}$ & Mixed bacterial assemblage & $\begin{array}{c}0.02 \mu \mathrm{m}-0.2 \mu \mathrm{m} \\
\text { C/DOM = internal } \\
\text { bacterial components } \\
\text { (not cell wall) }\end{array}$ & Tranvik (1994) \\
\hline $\begin{array}{c}\text { Pteridomonas danica } \\
\text { Patterson and Fenchel } \\
\text { (nanoflagellate) }\end{array}$ & Escherichia coli & $<\operatorname{l\mu m}$ & $\begin{array}{l}\text { Pelegri et al. } \\
\text { (1999) }\end{array}$ \\
\hline $\begin{array}{c}\text { Cafeteria sp. } \\
\text { Paraphysomonas } \\
\text { imperforata } \\
\text { (nanoflagellates) }\end{array}$ & Halomonas halodurans & $-\cdots$ & This study \\
\hline $\begin{array}{c}\text { Uronema sp. } \\
\text { (sucticociliate) }\end{array}$ & Halomonas halodurans & $-\cdots$ & This study \\
\hline
\end{tabular}

Table 4-1. Summary of the characteristics of "grazer-enhanced" C/DOM in cited investigations. Cultures discussed within the review of Nagata and Kirchman (1992a) are not cited here.

\subsubsection{Goals of the study.}

The overall goal of this study was to examine the DOM dynamics in cultures of different protozoan species. To achieve this goal, experiments were conducted with three different protozoan species, two flagellates and a ciliate. Population dynamics of both protozoa and prey were monitored as well as DOC and surfactant concentrations. The following specific questions were addressed:

1. What are the temporal dynamics of bulk DOC?

2. How do surface activity and lipid concentration vary in these conditions? 
3. How does surfactant production in protozoan cultures compare with previous estimates of phytoplankton surfactant production?

4. What factors affect surfactant production - protozoan species, ingestion rate, feeding efficiency, and/or prey growth substrate?

5. How do lipid components of DOM (lipopolysaccharides, bulk lipids) compare with surfactant concentrations?

6. What are bulk compositional characteristics of different components of "grazerenhanced" DOM in cultures?

\subsection{Methods}

\subsubsection{Organisms studied.}

The prey for all protozoan cultures was Halomonas halodurans, a ubiquitous marine bacterium, about $0.5 \mu \mathrm{m}$ in diameter. The protozoa compared in this study were Cafeteria sp., Paraphysomonas imperforata, and Uronema sp. Cafeteria sp. (clone: Cflag) and $P$. imperforata (clone: VS1) are both flagellates, approximately $2-4 \mu \mathrm{m}$ in diameter. Previous work indicated that Cafeteria was a very efficient grazer, producing large quantities of dissolved material (Barbeau, 1998). P. imperforata, on the other hand, produced large flocculent material that settled to the bottom of the culture flask (Barbeau, 1998). The ciliate in this study, Uronema sp., clone BBCil, is $10-15 \mu \mathrm{m}$ in size and has been shown to produce large enough quantities of surface-active material to interfere with stripping voltammetry experiments (Barbeau, 1998). This protist is a fast grazer and was used in Chapters 2 and 3. All organisms were obtained from the collection of D. Caron, University of Southern California, CA.

Bacterial prey was grown on three different growth media (Table 4-2). In most experiments, the growth media consisted of $0.04 \%$ yeast extract (YE) in sterile $0.2 \mu \mathrm{m}$ filtered Vineyard Sound seawater (VSW). This growth medium was used most frequently because it is a complex mix of organic compounds. Other growth media used were based on glucose and pyruvate. Each of these media included $\mathrm{NH}_{4} \mathrm{Cl}$ and $\mathrm{NaH}_{2} \mathrm{PO}_{4}$. The pyruvate media also included vitamins and the trace metals, $\mathrm{Fe}, \mathrm{Zn}$, Co and $\mathrm{Mn}$ to 
improve growth efficiency (K. Barbeau, unpublished data). Glucose-grown bacteria caused decreases in culture $\mathrm{pH}$ while pyruvate-grown bacteria did not (Barbeau, 1998).

With all growth media, bacterial prey were centrifuged and re-suspended in sterile Vineyard Sound seawater (VSW) three times to ensure complete removal of excess growth substrate (full method outlined in Chapter 2).

\begin{tabular}{|c|c|}
\hline Growth substrate & $\begin{array}{c}\text { Recipe } \\
\text { (per 1L VSW) }\end{array}$ \\
\hline Yeast Extract & $4 \mathrm{~mL}$ YE stock $(10 \% \mathrm{w} / \mathrm{v})$ \\
\hline Glucose & $\begin{array}{c}10 \mathrm{~mL} \text { glucose stock }(0.866 \mathrm{M}) \\
1 \mathrm{~mL} \mathrm{NH} \mathrm{NH}_{4} \text { stock }(6 \mathrm{~g} / 25 \mathrm{~mL}) \\
1 \mathrm{~mL} \mathrm{NaH}_{2} \mathrm{PO}_{4} \text { stock }(0.92 \mathrm{~g} / 25 \mathrm{~mL})\end{array}$ \\
\hline Pyruvate & $\begin{array}{c}10 \mathrm{~mL} \text { pyruvate stock }(3.1 \mathrm{M}) \\
1 \mathrm{~mL} \mathrm{NH}_{4} \mathrm{Cl} \text { stock } \\
1 \mathrm{~mL} \mathrm{NaH}_{2} \mathrm{PO}_{4} \text { stock } \\
1 \mathrm{~mL} \mathrm{~F} / 2 \text { vitamin solution }(\mathrm{Guillard} \text { and Ryther, } \\
1962) \\
1 \mathrm{~mL} \mathrm{FeCl}_{2}, \mathrm{ZnCl}_{2}, \mathrm{MnCl}_{2} \text { stocks }\left(10^{-4} \mathrm{M}\right) \\
10 \mu \mathrm{L} \mathrm{CoCl}{ }_{2} \text { stock }\left(10^{-4} \mathrm{M}\right)\end{array}$ \\
\hline
\end{tabular}

Table 4-2. Bacterial growth media.

Recipes for these growth media modified from Lim et al. (1993) (YE) and Barbeau (1998) (glucose and pyruvate).

\subsubsection{Protozoan cultures.}

In all experiments, prey concentrates were diluted with VSW to the desired volume and prey concentration. Protist inocula ( $5 \mathrm{~mL}$ - containing 500-1000 cells $/ \mathrm{mL})$ were added to each bacterial culture to begin an experiment. Initial studies were conducted with Uronema as the predator and H.halodurans as the prey. In these experiments, population and surfactant samples were taken approximately every 6 hours. In experiments involving flagellate cultures, samples for all parameters were taken every 8-10 hours. The timing for these experiments was chosen as a result of previous culture studies (Barbeau et al., submitted). Population samples were preserved with $0.01 \%(\mathrm{v} / \mathrm{v})$ glutaraldehyde. Dissolved $(<0.2 \mu \mathrm{m})$ samples were collected via syringe filtration through $0.2 \mu \mathrm{m}$ surfactant-free cellulose acetate filters (Nalgene, Fisher Scientific) and analyzed for surfactant, lipid, and DOC concentrations. A list of experiments discussed in this chapter is summarized in Table 4-3. 


\begin{tabular}{|c|c|c|c|c|}
\hline Date & Protist & $\begin{array}{l}\text { Bacterial growth } \\
\text { substrate }\end{array}$ & Parameters analyzed & Goal \\
\hline $\begin{array}{c}11 \\
12 / 98\end{array}$ & Uronema & Yeast extract & Protist \#, surfactants & Initial Study \\
\hline $12 / 98$ & Uronema & Glucose & Protist \#, surfactants & $\begin{array}{l}\text { Effect of growth } \\
\text { substrate }\end{array}$ \\
\hline $2 / 99$ & Uronema & Pyruvate & Protist \#, surfactants & $\begin{array}{l}\text { Effect of growth } \\
\text { substrate }\end{array}$ \\
\hline $3 / 99$ & $\begin{array}{c}\text { Uronema } \\
\text { Cafeteria } \\
\text { P. Imperforata }\end{array}$ & Yeast extract & $\begin{array}{l}\text { Protist \& bacteria \#'s, } \\
\text { surfactants, DOC, LPS }\end{array}$ & $\begin{array}{l}\text { Effect of protozoan } \\
\text { species; } \\
\text { Collection study }\end{array}$ \\
\hline $7 / 99$ & Uronema & Yeast extract & $\begin{array}{c}\text { Protist \& bacteria \#'s, } \\
\text { surfactants, DOC, } \\
\text { lipid }\end{array}$ & $\begin{array}{c}\text { Centrifugation } \\
\text { study }\end{array}$ \\
\hline $\begin{array}{c}7 / 99 \\
(3 \text { total })\end{array}$ & $\begin{array}{c}\text { Uronema } \\
\text { Cafeteria } \\
\text { P. Imperforata } \\
\end{array}$ & Yeast extract & $\begin{array}{c}\text { Protist \& bacteria \#'s, } \\
\text { DOC, lipid }\end{array}$ & Lipid dynamics \\
\hline
\end{tabular}

Table 4-3. List of experiments examined in this chapter.

\subsubsection{Parameter analyses.}

\subsubsection{Surfactants - Electrochemical method}

Surfactants were measured using an electrochemical method described by Hunter and Liss (1981). In this method, the reduction of $\mathrm{Hg}^{+2}$ to $\mathrm{Hg}^{0}$ on the surface of a dropping mercury electrode is measured over a range of potential. Surface-active material present in the sample inhibits this reduction, causing a decrease in the reduction peak height (see Figure 4-3 for an example). This method has been used extensively to measure surfactant concentrations in natural systems (Hunter and Liss, 1981; Zutic et al., 1981; Cosovic et al., 1985).

Samples were stored in the $4^{\circ} \mathrm{C}$ refrigerator. Immediately prior to analysis, $15 \mathrm{~mL}$ sample aliquots were spiked with $100 \mu \mathrm{L} 0.2 \mathrm{M} \mathrm{HgCl}_{2}$ (Sigma) in solvent-cleaned glass sample cups. Each sample was analyzed with a polarographic analyzer/ stripping voltammeter (EG\&G Princeton Applied Research) with the following program: purge with $\mathrm{N}_{2}(g)$ for $30 \mathrm{sec}$, ramp reduction potential between $0.05 \mathrm{~V}$ and $-0.4 \mathrm{~V}$ at $2 \mathrm{mV} / \mathrm{sec}$, drop size $=$ small, and drop time $=5 \mathrm{sec}$. The maximum peak height was measured with software written by N. Frew. 
The difference in peak height from an operationally-defined zero-surfactant control (sterile VSW) was assumed to be linearly related to the amount of surfactant in the sample. This difference was related to an external standard, Triton X-100 (polyoxyethylene t-octyl phenol, MW=600, Sigma). This method assumes that the aqueous molecular diffusion coefficient of the surface-active material in the sample and the standard (Triton X-100) are equivalent. Standard solutions of Triton X-100 ranged from $0.05 \mathrm{mg} / \mathrm{L}$ to $2.0 \mathrm{mg} / \mathrm{L}$ Triton X-100 in sterile VSW. This method did not give a linear response for surfactant concentrations higher than $2.0 \mathrm{mg} / \mathrm{L}$. Therefore, all samples that contained higher concentrations were diluted to elicit a response within the linear range of the method. Standard solutions were not stable due to high wall losses, especially in the most concentrated standards. Thus, standards were made monthly and analyzed the day after they were made. The stability of samples was also monitored and is discussed in section 4.3.2. In brief, samples were shown to be significantly more stable than Triton X-100 and thus stable over the storage period (up to six weeks in the refrigerator).

It must be stressed at this stage that surfactant concentrations are expressed in terms of Triton X-100 equivalents and so a direct comparison between surfactant and DOC concentrations is not appropriate. Direct comparison of TX-100 equivalents to organic carbon concentrations is not possible because one is an index of an "activity" and the other is a measure of mass. Identical TX-100 equivalents will be measured in samples containing high concentrations of material with few surface-active properties as in samples with low concentrations of highly surface-active material.

\subsubsection{Organic carbon}

Total and dissolved organic carbon (TOC and DOC) concentrations were measured using high temperature combustion (Peltzer and Brewer, 1993). Samples were analyzed at UMass-Boston with the help of Penny Vlahos, courtesy of Dr. R. Chen. For TOC and DOC analysis, aqueous samples were acidified with $50 \%(v / v) \mathrm{H}_{3} \mathrm{PO}_{4}(100 \mu \mathrm{L}$ acid per $20 \mathrm{~mL}$ sample) and bubbled with $\mathrm{N}_{2}$ to remove inorganic $\mathrm{CO}_{2}$. Three aliquots of 
the acidified sample $(50 \mu \mathrm{L})$ were then injected into a high-temperature combustion oven and the resultant $\mathrm{CO}_{2}$ was measured with a $\mathrm{Li}$-Cor $\mathrm{CO}_{2}$ analyzer. The average of the three injections was used to determine the organic carbon concentration. A four-point external standard curve was used to calculate the $\mathrm{CO}_{2}$ response factor for organic carbon quantitation. Milli-Q water blanks were used to monitor instrument conditions over the course of the sample run.

\subsubsection{Populations}

Populations were determined from the glutaraldehyde-preserved samples using epifluorescence microscopy of stained cells after the method in Lim et al. (full method outlined in Chapter 2). Briefly, samples were stained with acridine orange and drawn down onto $0.2 \mu \mathrm{m}$ black polycarbonate filters. Cells in 16 random fields on the filter were counted using epifluorescence microscopy under 1000X. In select cases, cells were counted via phase contrast.

\subsubsection{Lipopolysaccharides (LPS)}

Lipopolysaccharide (LPS) concentrations were measured in culture filtrates with a fluorometric assay (Associates of Cape Cod, Woods Hole, MA) based on the turbidometric method proposed in Watson et al. (1977) and modified by M. Dennett and D. Caron. This method relies on the reaction of LPS with Limulus amebocyte lysate, an aqueous extract of horseshoe crab blood. This reaction releases a chromophore, $p$ nitroanilide (pNA) which absorbs at $405 \mathrm{~nm}$. External standards are used to relate the $\log$ of the onset of pNA absorption to the log of LPS concentration.

All reagents used in this method were purchased from Associates of Cape Cod, Woods Hole, MA. All samples were stored in the refrigerator until analysis. Samples were diluted 1:66 and 1:121 (if necessary) for analysis. Analyses were performed in 96well microplates. Pyrogen-free water $(500 \mu \mathrm{L})$ and sample $(50 \mu \mathrm{L})$ were combined in each well. Standards and blanks were placed in columns at the end of the plate. Immediately prior to analysis, $50 \mu \mathrm{L}$ of lysate was added to each well. Plates were then 
placed in a plate reader for 1 hour or until all samples had saturated the detector. The plate reader measured absorbance at $405 \mathrm{~nm}$. Samples, standards and blanks were run at least twice.

\subsubsection{Lipids}

The lipid extraction method described here is modified from the method first published by Bligh and Dyer (1959). Aqueous samples were extracted with an equivalent volume of 2:1 $\mathrm{CHCl}_{3}: \mathrm{MeOH}$. This extraction was repeated twice (3X total). All chloroform phases were combined and back-extracted with an equivalent volume of Milli-Q water. The resultant chloroform phase was often cloudy due to a slight emulsion with water. Addition of $1-2 \mathrm{~mL} \mathrm{MeOH}$ removed this emulsion and cleared the extract. A tertiary azeotrope is formed by $\mathrm{MeOH}, \mathrm{CHCl}_{3}$, and water in the percent ratio 8.2: 90.5: 1.3 (boiling point $=52.3^{\circ} \mathrm{C}-\mathrm{CRC}$ ). All water was effectively removed from the chloroform phase by this azeotrope during roto-evaporation. However, to ensure that the chloroform phase was truly free of $\mathrm{MeOH}$ and water, $15 \mathrm{~mL}$ of chloroform was added to the evaporated extract of $1-2 \mathrm{~mL}$ and the extract was roto-vapped again to a final volume of $<1 \mathrm{~mL}$. The entire extract was dried in solvent-cleaned Sn cups for elemental analysis (Fisons Instrument EA1108 Elemental Analyzer). Culture filtrates were combined for lipid extraction to ensure that the samples were above the detection limit. Early studies indicated $150 \mathrm{~mL}$ of filtrate were needed to measure $50 \mu \mathrm{g} \mathrm{C}$ by elemental analysis. Therefore, samples from various protozoan grazing experiments were combined according to Table 4-5 (end of section 4.3.4).

\subsubsection{Iatroscan analyses}

Prior to elemental analysis, lipid extracts were analyzed by B. Bergen (US EPA, Narragansett, RI) for compositional information. These analyses were conducted on a Chromarod / Iatroscan® TLC-FID as detailed in Bergen et al. (1999). Sample extracts were reduced in volume to $<50 \mu \mathrm{L}$ for this analysis. Silica-gel coated glass rods were spotted with $1 \mu \mathrm{L}$ of sample and developed with two different solvents. The first 
developing step used 11:3:0.03 hexane: ether: acetic acid to separate non-polar lipids. The second step used 20:14:1 chloroform: methanol: water to separate polar lipids. After each developing step, the rods were burned with a $\mathrm{H}_{2}$ flame and the resultant ions were monitored by an FID detector. Lipids in samples were quantified by the external standards run concurrently. External standards included steryl/methyl ester (cholesterol palmitate), triacylglycerol (tripalmitin), free fatty acid (palmitic acid), sterol (cholesterol), phosphatidyl ethanolamine, lecithin, lysolecithin, and monoglycerol. Many peaks in these samples did not coincide with specific lipid standards. Therefore, total peak areas for non-polar and polar lipids were compared to indicate the type of lipid that was dominant in a particular sample.

\subsection{Results}

\subsubsection{Comparison of methods used for collection of dissolved samples.}

Three different methods were tested for ability to collect surface-active material reproducibly and accurately: syringe filtration, centrifugation and vacuum filtration. This test was performed at the last time point $(t=86 \mathrm{~h})$ of the interspecies comparison experiment in March 1999. In the first method, aliquots were syringe filtered using $0.2 \mu \mathrm{m}$ surfactant-free cellulose acetate filters. In the second, samples were centrifuged at $10,000 \mathrm{rpm}(11,180 \mathrm{Xg})$ for $45 \mathrm{~min}$ (centrifuge - Biofuge $22 \mathrm{R}$, Heraeus). This centrifugation is longer than the method used to separate bacteria from growth substrate so the supernatant should be similar to other "dissolved" samples. The third method was vacuum filtration $(<10 \mathrm{psi})$ through a $0.2 \mu \mathrm{m}$ polycarbonate filter (Nuclepore, Fisher Scientific). In general, syringe filtration allowed the most surfactants to pass into the operationally-defined "dissolved" phase and vacuum filtration allowed the least (Figure 4-4A). Syringe filtration was chosen to collect filtrates for headspace partitioning. However, this is an operationally-defined parameter. The different recoveries of surfaceactive material were most likely a function of the flow rate at which the sample was collected. Rupturing of the cells was considered a potential cause but other studies have shown syringe filtration is gentle enough for this purpose (Nagata and Kirchman, 1992a). 
It is more likely that some aggregates held together by this material were more easily broken by faster flow rates. However, it is not possible to unequivocally discount cell rupture as a cause of the variable surfactant concentrations in the syringe filtrations.

Samples from the collection method study were also analyzed for DOC and LPS concentrations (Figures 4-4B and 4-4C). The DOC concentrations showed similar behavior as the surfactant data for the most part. The highest observed concentrations occurred in the Uronema syringe-filtration and the lowest occurred in the H. halodurans vacuum-filtration. There was less variation between the collection methods for both flagellate cultures. The LPS concentrations did not show the enhancement in syringefiltration evident in either of the other two Uronema parameters. Oddly, the centrifugation method generated the most "dissolved" LPS with roughly equivalent concentrations present in syringe- and vacuum-filtration samples. This was also true in the $P$. imperforata cultures, though the enhancement was not as pronounced as in the Uronema cultures. Within error, all collection methods produced equivalent LPS concentrations in both the Cafeteria and H. halodurans filtrates.

In order to rule out episodic cell rupturing as a cause for potentially spurious surfactant maxima, syringe-filtered samples were compared against centrifuged samples in a Uronema culture. Syringe filtration may rupture cells due to the sheer of forcing water past the cells on the filter surface and the potential osmotic shock if the cells are exposed to air. Rupturing may occur during centrifugation due to centripetal sheer. However, trend parity in the two data sets would suggest a common underlying process that is not affected by potentially episodic processes such as cell rupturing.

This experiment was conducted with Uronema and H. halodurans grown on yeast extract growth medium. Uronema was used because it showed the greatest difference between syringe-filtration and other collection methods. Filtrate samples were analyzed in duplicate from duplicate bottles. Surfactant concentrations in syringe-filtered samples were consistently higher than those in centrifuged samples but the temporal trends observed in the samples are the same (Figure 4-5). Therefore, it is presumed for the 
remained of this thesis that data generated by syringe-filtration is robust and not the product of cell rupture or other episodic experimental artifacts.

Surfactant or organic matter contamination from different types of filters were monitored by the analysis of procedural blanks (40mL VSW through appropriate filter). Cellulose acetate filters (Whatman - Fisher Scientific) were observed to bleed large amounts of surfactant material. This bleed was systematic $(2.29 \pm 0.09 \mathrm{mg} / \mathrm{L}$ in the first $20 \mathrm{~mL}$ and $0.95 \pm 0.07 \mathrm{mg} / \mathrm{L}$ in the second $20 \mathrm{~mL}$ ) and could be subtracted from samples

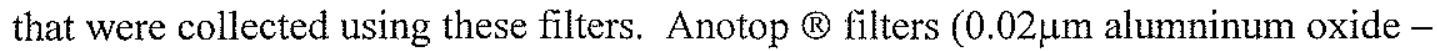
Whatman, Fisher Scientific) used in the size-fractionation study were also observed to bleed surface-active organic material. All other filter types - vinyl (Gelman, Fisher Scientific), Teflon (Nalgene, Fisher Scientific), and polysulfone (Gelman Acrodisc ${ }^{\circledR}$, Fisher Scientific) - were found to have no significant contamination problems. Filters were not cleaned so the effect of different cleaning procedures on contamination problems was not ascertained.

\subsubsection{Storage experiments}

\subsubsection{Surfactant samples}

Samples from a Uronema culture were stored in the refrigerator at $4^{\circ} \mathrm{C}$ for different lengths of time to test the stability of the surface-active material. Twelve $25 \mathrm{~mL}$ samples were collected for both the ciliate and bacterial cultures at the beginning, midway and the end of the $48 \mathrm{~h}$ grazing experiment. Two samples were analyzed immediately and the remainder of the samples were stored in the refrigerator. Two samples were sacrificed at Day 1, Day 2, Day 4, Day 7 and Day 21. Samples from the beginning of the experiment were the most variable (Figure 4-6) in both the ciliate and bacterial cultures. Subsequent H. halodurans samples were stable and varied little from the initial value. Uronema samples showed more variability. However, there was no systematic decrease in concentration over storage time. Samples were deemed stable for the duration of the storage experiment. 


\subsubsection{Lipopolysaccharide storage experiment}

Samples from a $H$. halodurans bacterial control were collected to test the effect of storage method and time on LPS concentrations. Samples were analyzed using the LPS method described above. Duplicate samples were analyzed immediately after collection and then stored in the freezer and the refrigerator for 16 days and 30 days. Samples were freeze-thawed and did not have to be sacrificed because volume requirements for the LPS assay were so small. Thus sample-to-sample heterogeneity was not a concern in this case. Initial measurements of H. halodurans filtrates were the same for all four samples (Figure 4-7). After 16 and 30 days, the refrigerated samples maintained constant LPS concentrations. However, the LPS concentrations doubled in the frozen samples. There was no difference between concentrations measured at 16 and 30 days. The cause of this marked increase in LPS concentration is unknown. Freezing and subsequent thawing may have disrupted aggregated particles or small cells which then exposed more "active" sites for the LPS assay.

\subsubsection{Initial studies with Uronema and H.halodurans}

\subsubsection{Yeast extract growth medium}

Initial experiments were conducted with a ciliate, Uronema, and H. halodurans as prey. The bacteria were grown on YE for each of these experiments. Surfactant and protist concentrations were monitored for 48-65h (Figure 4-8). These initial experiments showed production of surface-active material in the middle of the experimental time course when protozoan were in late exponential growth. In each of these experiments, the maximum surfactant concentration occurred at the end of the protist exponential growth phase: Once the protists were in stationary growth, surfactant concentrations decreased to near background levels. Surface-active material remained low in both these experiments. The maximum surfactant concentration in the first experiment (Figure 48A) was approximately two-fold higher than in the second experiment (Figure 4-8B). Protist concentrations were also twice as high in the first experiment. 


\subsubsection{Glucose and pyruvate-based media}

The effect of bacterial composition on surfactant production was tested by changing the bacterial growth medium. Unlike organic-rich media such as yeast extract, glucose or pyruvate-based growth media require bacteria to manufacture complex organic compounds. Past experience (myself and K. Barbeau) has shown that bacteria grown on media like glucose or pyruvate are less sticky than those grown on yeast extract. This stickiness could be related to the cell surface composition and could thus affect surfactant production in protozoan grazing cultures. In the glucose experiment, the same basic behavior was observed as in the YE-bacteria experiments described above (Figure 4-9). However, the maximum surfactant concentration was much lower while the protist population was approximately the same. Similar results were observed when bacteria were grown on pyruvate (Figure 4-10).

\subsubsection{Interspecies comparison}

The effect of protozoan species on surfactant production was the subject of an interspecies comparison experiment conducted in March 1999. The ciliate, Uronema, was compared to two flagellates, Cafeteria sp. and $P$. imperforata. All protists were fed the same batch of prey. Previous work inferred from differing Th:C ratios that separate batches of bacteria could have different surface compositions even though growth conditions were as similar as possible (Barbeau, 1998). Both bacterial and protist population dynamics were analyzed in all cultures. Dissolved organic carbon, lipopolysaccharide, and surfactant concentrations were also monitored. The bacterial population remained relatively constant over the experimental time course (Figure 4-11). DOC concentrations dropped initially, potentially due to bacterial utilization but remained constant thereafter. Surfactant concentrations stayed low. LPS concentrations were constant (approximately $0.025 \mathrm{mg} / \mathrm{L}$ ) as compared to surfactants and DOC concentrations.

In the Uronema cultures, bacterial cells were grazed to near threshold levels (approximately $10^{6}$ cells $/ \mathrm{mL}$ ) and protist numbers increased rapidly (Figure 4-12). 
Dissolved organic carbon (DOC) concentrations remained relatively constant after an initial drop (with the notable exception of $t=85 \mathrm{~h}-$ no explanation is possible at this stage). While the protist population was in exponential growth, surfactant activities increased dramatically. Surfactant activities dropped precipitously once the protist population reached stationary growth. Secondary peaks of surfactant activity in this culture were due to subsequent crash-and-boom cycles in the protozoan population. LPS concentrations were again low $(0.05 \mathrm{mg} / \mathrm{L})$ relative to DOC and surfactants $(\leq 5 \% \mathrm{DOC})$.

Cafeteria did not efficiently graze the bacteria to $10^{6} \mathrm{cell} / \mathrm{s} / \mathrm{mL}$ even though protist numbers increased over the time course of the experiment (Figure 4-13). DOC concentrations decreased in the first 12 hours but remained constant for the remainder of the experiment with the exception of $t=86 \mathrm{~h}$. Surfactant activities increased steadily with time until $t=36 \mathrm{~h}$ resulting in a two-fold increase over the initial activity. However, on average, concentrations were lower than in the ciliate cultures. LPS concentrations were again low $(0.03 \mathrm{mg} / \mathrm{L})$.

Paraphysomonas imperforata showed efficient removal of bacterial prey and significant increases in population over the $86 \mathrm{~h}$ experiment (Figure 4-14). DOC concentrations decreased early in the experiment but remained relatively constant from that point onward. Surfactant concentrations increased more than two-fold during the experiment. Peaks in surfactant activity were observed during the latter part of the experiment. As in the Cafeteria and Uronema cultures, the onset of surfactant production coincided with rapid protozoan growth. LPS was low $(0.06 \mathrm{mg} / \mathrm{L})$ and constant.

This experiment compared the relative surfactant production capabilities of the three protists studied, given a common bacterial prey. Surfactant activities were plotted versus time for all species in Figure 4-15. By visual inspection one can see that surfactant activities were highest in the Uronema cultures and lowest in the bacterial controls. The greatest changes were observed in the Uronema culture. The flagellates produced less surface-active material but instead of decreasing precipitously, this material persisted after protists reached stationary growth. Both flagellate species reached maximal concentrations significantly later than the ciliate. Protistan populations 
also reached maxima later in the two flagellate cultures. Therefore, significant surfactant production was coincident with protozoan exponential growth in all cultures, implying the timing of surfactant production was related in some way to protozoan growth stage.

This dynamic behavior was not observed in the DOC concentrations, suggesting that the two cycles are not tightly coupled (Figure 4-16). For all species, DOC concentrations decreased during the first 12 hours of the experiment and remained relatively constant thereafter. This behavior was consistent with previous work (Barbeau, 1998; Barbeau et al., submitted). The average concentration of DOC was the same in all cultures including the bacterial control. The decrease in initial DOC was most likely explained by bacterial utilization. It is possible that increases in "grazer-enhanced" DOM were not observed because the background DOC was so high. When bacterial concentrations were approximately $10^{7}$ cells $/ \mathrm{mL}$, bacterial biomass could represent up to $2 \mathrm{mgC} / \mathrm{L}$ (from $10^{7}$ cells $/ \mathrm{mL}^{*} 200 \mathrm{fgC} /$ cell - bacterial $\mathrm{C}$ value from $\mathrm{D}$. Caron, personal communication). If protists release $20 \%$ of this material as DOC, the "grazer-enhanced" DOM would contribute only $\sim 0.4 \mathrm{mgC} / \mathrm{L}$ to the background DOC of $4-6 \mathrm{mgC} / \mathrm{L}$. In addition, potential bacterial utilization of this material could further decrease the apparent production of "grazer-enhanced" DOM. This estimate of "grazer-enhanced" DOM production also highlights the discrepancy between surface activity as expressed by mass Triton X-100 equivalents and the mass of DOC in the culture.

LPS concentrations were similar in all cultures studied (Figure 4-17). Concentrations varied a great deal over the time course of the experiment within a small range (note y-axis - $\leq 5 \% \mathrm{DOC}$ ). By the end of the experiment, the highest concentrations were observed in the $P$. imperforata culture, but the difference between this culture and the others was not significant.

\subsubsection{Ingestion and surfactant production rates}

Ingestion rates were calculated for experiments with the following formula (from Frost (1972) modified by Heinbokel (1978)): 


$$
\text { (1) IR }=\frac{B a c t_{1}-\text { Bact }_{2}}{\left(P_{2}-P_{1} / \ln P_{2}-\ln P_{1}\right) *\left(t_{2}-t_{1}\right)}
$$

where $I R=$ ingestion rate (bacteria / protist $/ \mathrm{h}$ ), Bact $t_{\mathrm{t}}=$ bacteria concentration at time point $\mathrm{n}($ cells $/ \mathrm{mL}), \mathrm{P}_{\mathrm{n}}=$ protist concentration at $\mathrm{n}($ cells $/ \mathrm{mL})$, and $t=$ time $(\mathrm{h})$. I then used the ingestion rate formula as the basis for calculating surfactant production rates. This formulation is ideal because it accounts for increasing protozoan populations. Therefore, surfactant production rates were calculated with the formula based on equation 1:

$$
\text { (2) } S P R=\frac{\operatorname{Surf}_{2}-\text { Surf }_{1}}{\left(P_{2}-P_{1} / \ln P_{2}-\ln P_{1}\right) *\left(t_{2}-t_{1}\right)}
$$

where SPR $=$ surfactant production rate $(\mathrm{mg} / \mathrm{L} / \mathrm{h})$ and $\operatorname{Surf}_{\mathrm{ll}}=$ surfactant concentration at time point, $n(\mathrm{mg} / \mathrm{L})$. The maximum IR and SPR over time are compared for the three different protozoan species in Figure 4-18. In addition, maximum IR is compared to maximum surfactant activity, initial prey concentration, and protozoan species in Table

\begin{tabular}{|c|c|c|c|c|}
\hline Protist & $\begin{array}{l}\text { Prey growth } \\
\text { substrate }\end{array}$ & $\begin{array}{c}\text { Initial prey } \\
\text { concentration } \\
\left(10^{7} \text { cells } / \mathrm{mL}\right)\end{array}$ & $\begin{array}{l}\text { Maximum IR } \\
\text { (cells/prot/h) }\end{array}$ & $\begin{array}{l}\text { Maximum surfactant } \\
\text { activity (mg/L TX100) }\end{array}$ \\
\hline Uronema & $\begin{array}{c}\text { Yeast extract } \\
" \\
"\end{array}$ & $\begin{array}{l}1.27 \\
1.01 \\
1.33\end{array}$ & $\begin{array}{l}91^{\mathrm{a}} \\
721 \\
876\end{array}$ & $\begin{array}{l}5.81 \\
3.40 \\
3.04 \\
\end{array}$ \\
\hline Uronema & --- & --- & $1580^{\circ}$ & --- \\
\hline Uronema & $-\cdots$ & --- & $180-420^{\mathrm{c}}$ & $\cdots$ \\
\hline Uronema & -- & $\cdots$ & $80-720^{\mathrm{d}}$ & $\cdots$ \\
\hline Cafeteria & Yeast extract & 1.27 & 29 & 2.58 \\
\hline P. imperforata & Yeast extract & 1.27 & 166 & 3.23 \\
\hline
\end{tabular}
4-4.

Table 4-4. Maximum ingestion rates and activities for protists studied. Ingestion rates were calculated using the equation 1 and are compared to literature values for the same species (Uronema only). Maximum surfactant activities do not necessarily coincide with the time period of maximum ingestion rate (compare Figures 4-15 and 4-18).

${ }^{a}$ - potentially underestimated.

b - from Taylor et al. (1985)

c - from Wallberg et al. (1997)

d- from Iriberri et al. (1995)

Maximum ingestion rates and surfactant production rates rarely coincided in time, though in most cultures the maximum surfactant production rate occurred after but within 
one or two time points of the maximum ingestion rate. This is consistent with the earlier observation that surfactant production was occurring during the transition from exponential to stationary growth. As expected, the maximum surfactant production rate occurred in the Uronema cultures. Both Cafeteria and Uronema cultures had one large surfactant production rate peak that was significantly greater than surfactant production rates at all other time points. In contrast, peak surfactant production rates in the $P$. imperforata culture were approximately constant for 3 or 4 time points (Figure 4-18).

\subsubsection{Lipid data-bulk and compositional information.}

Samples were combined according to Table 4-5 for analysis by Iatroscan $®$ and CHN. In general, the concentration of lipids in the bulk sample was quite low $(\leq 10 \%$ of DOC) relative to DOC concentrations. This is consistent with the LPS results discussed earlier. In most of the samples, nonpolar lipids predominated as seen by NP/P ratios greater than 1 (Table 4-5). Sample peaks that co-eluted with concurrent external standards were quantified where possible. Steryl/methyl esters, free fatty acids, and sterols were present in a number of samples. Polar lipids predominated in some samples but only slightly because the NP/P ratios were very close to 1 . The monoglyceride / AMPL (acetone-mobile-polar-lipid) peak was present in most of the grazing samples run. However, no quantifiable phospholipid peaks were observed in any of the samples. It is possible that phospholipids within the sample did not co-elute with the phophatidylcholine standard (phosphatidyl-ethanolamine). However, the lack of appreciable phospholipids raises concerns about the applicability of the Nagata-Kirchman model of phospholipid-rich "grazer-enhanced" DOM to these systems.

Samples were combined according to the chart to increase sample size. Lipid class concentrations were determined from Iatroscan analyses (by B. Bergen) of $1 \mu \mathrm{L}$ $\mathrm{CHCl}_{3}$ extract and normalized to the original sample volume $(\mathrm{n} / \mathrm{d}=$ not detected $)$. In many cases, peaks did not co-elute with standards and so full compositional information was not attained. Standards included steryl / methyl ester (SE), free fatty acid (FFA), 


\begin{tabular}{|c|c|c|c|c|c|c|c|c|c|}
\hline & $\begin{array}{l}\text { Time } \\
\text { Points }\end{array}$ & $\begin{array}{c}\text { SE } \\
(\mu \mathrm{g} / \mathrm{mL})\end{array}$ & $\begin{array}{c}\text { FFA } \\
(\mu \mathrm{g} / \mathrm{mL})\end{array}$ & $\begin{array}{c}\text { ST } \\
(\mu \mathrm{g} / \mathrm{mL})\end{array}$ & $\begin{array}{c}\text { MG/AMPL } \\
(\mu \mathrm{g} / \mathrm{mL})\end{array}$ & $\begin{array}{c}\text { NP area } \\
(\mathrm{mV})\end{array}$ & $\begin{array}{c}\text { P area } \\
(\mathrm{mV})\end{array}$ & $\begin{array}{l}\text { Ratio } \\
\text { NP/P }\end{array}$ & $\begin{array}{l}\text { Total lipids } \\
(\mathrm{mg} \mathrm{C} / \mathrm{L})\end{array}$ \\
\hline & $\begin{array}{c}\text { t0,t8,t15 } \\
7 / 19\end{array}$ & $\mathrm{n} / \mathrm{d}$ & 0.04 & 0.06 & 0.22 & 26.60 & 29.60 & 0.90 & 0.28 \\
\hline \multirow{3}{*}{5} & $\begin{array}{c}\mathrm{t} 23, \mathrm{t} 35, \mathrm{t} 48 \\
7 / 19\end{array}$ & $n / d$ & $n / d$ & $\mathrm{n} / \mathrm{d}$ & $n / d$ & $\mathrm{n} / \mathrm{d}$ & $\mathrm{n} / \mathrm{d}$ & $\mathrm{n} / \mathrm{d}$ & 0.21 \\
\hline & $0.2 \mu \mathrm{m} 10 / 3$ & $\mathrm{n} / \mathrm{d}$ & 0.09 & $n / d$ & 0.15 & 12.10 & 4.10 & 2.95 & lost \\
\hline & $0.02 \mu \mathrm{m} 10 / 3$ & $\mathrm{n} / \mathrm{d}$ & $\mathrm{n} / \mathrm{d}$ & 0.12 & 1.15 & 6.00 & 5.20 & 1.15 & lost \\
\hline \multirow{8}{*}{$\stackrel{\Xi}{\stackrel{5}{3}}$} & $\begin{array}{c}\mathrm{t} 0, \mathrm{t} 8, \mathrm{t} 15, \mathrm{t} 23 \\
7 / 19\end{array}$ & $n / d$ & $\mathrm{n} / \mathrm{d}$ & 0.10 & 0.16 & 19.90 & 5.60 & 3.55 & 0.24 \\
\hline & $\begin{array}{c}\mathrm{t} 35, \mathrm{t} 48, \mathrm{t} 60, \mathrm{t} 74 \\
7 / 19\end{array}$ & $n / d$ & $\mathrm{n} / \mathrm{d}$ & 0.08 & 0.06 & 8.20 & 4.40 & 1.86 & 0.17 \\
\hline & $\begin{array}{c}\mathrm{t} 0, \mathrm{t} 12, \mathrm{t} 22, \mathrm{t} 36 \\
7 / 29-\text { rep } 1\end{array}$ & 0.02 & $\mathrm{n} / \mathrm{d}$ & 0.11 & 0.15 & 12.10 & 8.20 & 1.48 & 0.28 \\
\hline & $\begin{array}{c}\mathrm{t} 0, \mathrm{t} 12, \mathrm{t} 22, \mathrm{t} 36 \\
7 / 29-\operatorname{rep} 2\end{array}$ & $\mathrm{n} / \mathrm{d}$ & $n / d$ & 0.12 & 0.06 & 14.00 & 4.80 & 2.92 & 0.25 \\
\hline & $\begin{array}{c}\mathrm{t} 46, \mathrm{t} 60, \mathrm{t} 70, \mathrm{t} 86 \\
7 / 29-\text { rep } 1\end{array}$ & $\mathrm{n} / \mathrm{d}$ & $\mathrm{n} / \mathrm{d}$ & $\mathrm{n} / \mathrm{d}$ & $n / d$ & $n / d$ & 2.20 & 0.00 & 0.13 \\
\hline & $\begin{array}{c}\mathrm{t} 46, \mathrm{t} 60, \mathrm{t} 70, \mathrm{t} 86 \\
7 / 29-\text { rep } 2\end{array}$ & $n / d$ & $\mathrm{n} / \mathrm{d}$ & 0.08 & 0.05 & 16.80 & 6.70 & 2.51 & 0.19 \\
\hline & $0.2 \mu \mathrm{m} 10 / 11$ & 0.13 & $n / d$ & 0.16 & 0.23 & 7.00 & 9.30 & 0.75 & 0.23 \\
\hline & $0.02 \mu \mathrm{m} 10 / 11$ & $\mathrm{n} / \mathrm{d}$ & $\mathrm{n} / \mathrm{d}$ & $\mathrm{n} / \mathrm{d}$ & 0.30 & $\mathrm{n} / \mathrm{d}$ & 11.60 & 0.00 & 0.19 \\
\hline \multirow{5}{*}{ 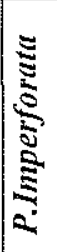 } & $t 0, t 12, t 24-8 / 9$ & $\mathrm{n} / \mathrm{d}$ & $n / d$ & 0.09 & 0.10 & 17.60 & 4.70 & 3.74 & 0.29 \\
\hline & $\mathrm{t} 36, \mathrm{t} 48, \mathrm{t} 61-8 / 9$ & 0.04 & $n / d$ & 0.06 & 0.13 & 22.30 & 7.40 & 3.01 & 0.26 \\
\hline & $\mathrm{t} 73, \mathrm{t} 85, \mathrm{t} 95-8 / 9$ & $n / d$ & $\mathrm{n} / \mathrm{d}$ & 0.08 & 0.20 & 7.20 & 7.60 & 0.95 & 0.25 \\
\hline & $0.2 \mu m-10 / 18$ & $\mathrm{n} / \mathrm{d}$ & $n / d$ & $\mathrm{n} / \mathrm{d}$ & 0.13 & $\mathrm{n} / \mathrm{d}$ & 3.90 & 0.00 & 0.18 \\
\hline & $0.02 \mu m-10 / 18$ & $\mathrm{n} / \mathrm{d}$ & $\mathrm{n} / \mathrm{d}$ & $n / d$ & $\mathrm{n} / \mathrm{d}$ & $\mathrm{n} / \mathrm{d}$ & $\mathrm{n} / \mathrm{d}$ & $\mathrm{n} / \mathrm{d}$ & 0.23 \\
\hline 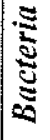 & all samples $-8 / 9$ & 0.07 & 0.06 & 0.09 & 0.14 & 10.60 & 5.90 & 1.80 & 0.33 \\
\hline
\end{tabular}

Table 4-5. Bulk and compositional lipid data for protozoan and bacterial samples.

sterol (ST), and monoglyceride (MG) / acetone-mobile-polar-lipid (AMPL). These same extracts were run on the $\mathrm{CHN}$ for elemental analysis. The concentration of $\mathrm{C}$ (per unit sample volume) is shown. The ratio of non-polar to polar lipids was calculated by dividing the total area in the non-polar lipid fraction $(\mathrm{mV})$ by the total area in the polar lipid fraction. 


\subsection{Discussion}

\subsubsection{Relative dynamics of organic carbon, surfactants and lipids.}

The dissolved organic carbon (DOC) dynamics observed in our study compared well with previous work performed in this laboratory (Barbeau, 1998). High initial DOC concentrations were potentially due to bacterial exudation after centrifugation and resuspension in sterile VSW. DOC concentrations then decreased precipitously, probably as a result of bacterial uptake and utilization. As mentioned above, the surfactant and lipid concentrations did not behave the same way as the bulk DOC. The lipid concentrations were never a significant fraction of the DOC $(\leq 10 \%)$. It was not surprising that different components of bulk DOC may vary independently since the origin of different components will affect the extent to which grazers can influence its release. In that vein, it is worth speculating on mechanistic explanations for the behavior of the surfactants and lipids in this system.

\subsubsection{Surfactants}

\subsubsection{Protozoan vs. phytoplankton production}

The surfactant material produced in most of the grazing cultures was greater than or comparable to activities of surfactant material attributed to phytoplankton in previous studies (Zutic et al., 1981; Vojvodic and Cosovic, 1996) (all data relative to TX-100Table 4-6). Studies by Zutic et al. (1981) measured surfactant activities as high as $6 \mathrm{mg} / \mathrm{L}$ TX-100 equivalents in unfiltered cultures of Skeletonema and Cryptomonas. Filtered samples $(>1.2 \mu \mathrm{m})$ had maximum surfactant activities of $4 \mathrm{mg} / \mathrm{L}$ TX-100 equivalents. Elevated surfactant activities appeared after phytoplankton entered stationary growth. In another study, a surfactant concentration of $18 \mathrm{mg} / \mathrm{L}$ TX-100 equivalents was measured in a culture of Phaeodactylum tricornutum (Vojvodic and Cosovic, 1996) but the DOC concentration in this diatom culture was $16.3 \mathrm{mg} / \mathrm{L}$, much higher than the DOC concentrations in my cultures $(2-6 \mathrm{mg} / \mathrm{L})$. Maximum surfactant activities in my cultures ranged from 2 to $7 \mathrm{mg} / \mathrm{L}$ TX100 equivalents with the highest activities observed in the ciliate treatments. Surfactant activities in bacterial controls 
were relatively stable at $1 \mathrm{mg} / \mathrm{L} \pm 0.5 \mathrm{mg} / \mathrm{L}$. Therefore, production by bacteria in the absence of grazers was negligible relative to the surfactants produced during grazing activity.

\begin{tabular}{|c|c|c|}
\hline $\begin{array}{c}\text { Surfactant activity } \\
\text { (mg/L TX100 equiv's) }\end{array}$ & Site (season) & Reference \\
\hline $0.015-0.475$ & North Adriatic Sea (1985-1993) & Vojvodic \& Cosovic (1996) \\
\hline $0.07-0.16$ & $\begin{array}{l}\text { North Adriatic Sea } \\
\text { (May \& Nov 1992) }\end{array}$ & Gasparovic \& Cosovic (1994) \\
\hline $\begin{array}{l}0.4 \text { (surface) } \\
0.8(100 \mathrm{~m})\end{array}$ & $\begin{array}{l}\text { Western Mediterranean } \\
\text { (April 1981) }\end{array}$ & Cosovic et al. (1985) \\
\hline $0.8->10$ & $\begin{array}{l}\text { Polluted harbors along Adriatic } \\
\text { coast }(1976-1979)\end{array}$ & Cosovic et al. (1985) \\
\hline $\begin{array}{c}0.97 \\
(\mathrm{DOC}=12.3 \mathrm{mg} / \mathrm{L})\end{array}$ & Prorocentrum micans & Zutic et al, (1981) \\
\hline $0.97-1.96$ & North Adriatic Sea (Oct 1979) & Cosovic et al. (1985) \\
\hline 1.95 & Dunaliella tertiolecta & Cosovic \& Vojvodic (1989) \\
\hline $\begin{array}{c}2.58 \\
(\mathrm{DOC}=3.87 \mathrm{mg} / \mathrm{L})\end{array}$ & Cafeteria, sp. & This study \\
\hline $\begin{array}{c}3.23 \\
(\mathrm{DOC}=3.68 \mathrm{mg} / \mathrm{L})\end{array}$ & Paraphysomonas imperforata & This study \\
\hline $3.4->10$ & $\begin{array}{l}\text { Oil-polluted surface microlayer } \\
\text { Rijeka Bay, Adriatic Sea } \\
\text { (1977-78) }\end{array}$ & Cosovic et al. (1985) \\
\hline $\begin{array}{c}5.8 \\
(\mathrm{DOC}=4.83 \mathrm{mg} / \mathrm{L})\end{array}$ & Uronema & This study \\
\hline 6.5 (max-unfiltered culture) & Cryptomonas, sp. & Zutic et al. (1981) \\
\hline 7 (max-unfiltered culture) & Skeletonema costatum & Zutic et al. (1981) \\
\hline$>10($ surface $)$ & $\begin{array}{l}\text { North Adriatic Sea } \\
\text { (bloom - Aug 1977) }\end{array}$ & Zutic et al. (1981) \\
\hline $\begin{array}{c}18 \\
(\mathrm{DOC}=16.3 \mathrm{mg} / \mathrm{L})\end{array}$ & Phaeodacilum tricornutum & Vojdovic et al. (1996) \\
\hline
\end{tabular}

Table 4-6. Measured surfactant activities in seawater, cultures and this study. 
Elevated surfactant concentrations have been observed during and immediately after phytoplankton blooms (Sakugawa and Handa, 1985). This observation coupled with data from cultures has led to the consensus that phytoplankton are the dominant source of surface-active material to the marine environment. However, data from this study suggests that protozoan grazing processes should also be considered sources of surface-active material. As active members of the microbial loop, grazers can constitute the primary source of surface-active material to oligotrophic ecosystems. This point can be illustrated with the following "back-of-the-envelope" calculation. Using the data for Cryptomonas from Zutic et al. (1981) in equation 2, I estimated a maximum surfactant production rate of $10^{-7} \mathrm{mg} / \mathrm{L} / \mathrm{cell} / \mathrm{h}$ for phytoplankton. Under bloom conditions $\left(10^{5}\right.$ cells/mL - R. Green, personal communication), a phytoplankton assemblage can produce surface-active material at $10^{-2} \mathrm{mg} / \mathrm{L} / \mathrm{h}$. This can be compared to the data presented in this chapter for protozoa. The maximum surfactant production rate was $10^{-5}$ to $10^{-6}$ $\mathrm{mg} / \mathrm{L} / \mathrm{cell} / \mathrm{h}$, depending on the protozoan species. Under non-bloom conditions $\left(10^{3}\right.$ cells $/ \mathrm{mL}$ ), protozoa can produce surface-active material at $10^{-2}$ to $10^{-3} \mathrm{mg} / \mathrm{L} / \mathrm{h}$. Since phytoplankton production is episodic (depending on presence or absence of bloom conditions, nutrient concentrations and light levels) and protozoan production is relatively constant, protozoa need to be considered a large source of surface-active material to microbial-loop dominated systems.

\subsubsection{Production mechanisms}

There is no direct evidence that the surface-active material produced in these cultures was actually synthesized by the protozoa themselves. Therefore, for the purposes of this discussion, I assumed that the surface-active material was "grazerenhanced" as defined by Taylor et al. (1985). The production of surface-active material should be influenced by a number of factors including protozoan species, ingestion rate, feeding mechanism, assimilation efficiency, digestive chemistry, and bacterial concentration and composition. The interspecies comparison experiment indicated that 
ingestion rates, surfactant production rates, and maximum surfactant concentrations were highest in the ciliate cultures.

The ciliate in our study is a filter feeder, beating its cilia to force water and particles towards its cytosome (oral area). Previous work in our laboratory suggested that the ciliate is an inefficient feeder and releases large quantities of dissolved surface-active material (Barbeau, 1998). However, the maximum ingestion rate and maximum surfactant production rates did not coincide in time. Instead, maximum surfactant production rates occurred after the maximum ingestion rate. This type of behavior is consistent with the surfactant production concept suggested by Zutic et al. (1981) aggregation of smaller compounds. Yet the loss of this material occurs quickly in the ciliate cultures, implying that the material is very labile and can be utilized easily by bacteria. Zutic et al. (1981) predicted that the aggregated material would be more refractory than the smaller compounds and so would remain in the water column long enough to be concentrated in the surface microlayer. Since this material seems rather labile to biological degradation, aggregation is an unlikely production mechanism. In all likelihood, the material is partially digested bacteria. Jumars et al. (1989) noted that high ingestion rates and poor assimilation efficiencies led to an increase in partially digested egesta. This material would be more labile than whole cells and so would be quickly degraded by bacterial consumption. This is consistent with results from field experiments with protozoan grazers (Barbeau and Moffett, submitted). Wall losses of radiotracers were high in treatments containing organisms in the $1-20 \mu \mathrm{m}$ size range. However, these wall losses decreased significantly when organisms between 1 and $20 \mu \mathrm{m}$ were removed. Wall losses were presumed to be indicative of the production of surfaceactive material (Barbeau and Moffett, submitted).

The effect of bacterial composition on surfactant production can be examined by either using two different prey organisms or altering the growth substrate of the bacteria. Early experiments used different growth substrates for the prey organism. Initially, experiments with prey grown on simple carbon sources such as glucose or pyruvate indicated that surfactant concentrations would be markedly lower. Previous work had 
noted that bacteria grown on yeast extract were stickier than those grown on glucose or pyruvate. These two observations led to the conclusion that the cell surfaces of bacteria grown on simple carbon sources were less surface-active than those grown on yeast extract. If the production of surfactant material was related to incomplete digestion of bacterial prey, it is likely that differences in cell-surface hydrophobicity would lead to changes in surfactant properties in the "grazer-enhanced" DOM. Later experiments indicated that high surfactant concentrations with YE-grown bacteria were not always present, implying that a complex media such as YE does not yield the same cell surfaces each time. Statistically relevant comparisons between cultures with bacteria grown on different growth substrates are not possible because there were too few glucose and pyruvate-bacteria cultures.

The flagellate, $P$. imperforata, is a combination filter and raptorial feeder (Barbeau, 1998), a cross between the ciliate, a true filter feeder, and Cafeteria, a true raptorial feeder. In this culture, the maximum ingestion rate and the maximum surfactant production rate coincided. However, the surfactant production rate was approximately constant over the time of exponential protozoan growth. Once the protozoa reached stationary growth, surfactant production rates decreased and surfactant activities stabilized. Because heat-killed bacteria were not used, I could not determine whether the constant surfactant activities were due to a balance between production and consumption or due to a cessation of surfactant production without concomitant utilization. It is tempting to suggest that the material produced by $P$. imperforata grazing is more refractory than that produced by the ciliate. However, neither flagellate culture was run long enough to ascertain whether the surfactant concentrations would decrease in time.

The second flagellate studied, Cafeteria, is a true raptorial feeder and would be expected to exhibit particle selectivity (Barbeau, 1998). Since its feeding strategy relied on particle interception, the ingestion rates were lowest for this flagellate. The maximum surfactant production rate, though, was comparable to that of $P$. imperforata. The feeding efficiency was similar in the two flagellates. It is possible that feeding behavior determined the relative amount of surfactant produced among the three protozoa studied. 
Protozoan food vacuoles are dynamic chemical micro-environments. The digestive process involves a drop in vacuole $\mathrm{pH}$ (to 2), fusion with lysozymes ( $\mathrm{pH} 5$ ), and vigorous enzymatic activity (Fok et al., 1982). The acidic vacuole chemistry has been implicated in the production of bio-available $\mathrm{Fe}^{+2}$ from colloidal iron oxide phases (Barbeau, 1998). In future experiments, the timing of $\mathrm{Fe}^{+2}$ production in grazing experiments can be compared to the timing of surfactant production in our grazing cultures. If the timing is similar, it is circumstantial evidence that acidic vacuole chemistry is playing a role in the production of surface-active DOM.

\subsubsection{Lipopolysaccharides and lipids}

Lipopolysaccharides were monitored in these cultures to see if these data were consistent with the hypothesis of Nagata and Kirchman (1992b) - "grazer-enhanced" colloidal material derived from bacterial cell membranes and should be phospholipid-rich - and Tranvik (1994) - "grazer-enhanced" colloidal material derived from internal bacterial cell components. LPS as measured by the assay described above should be derived from bacterial cell walls and outer membranes. A marked increase in LPS concentration with grazing activity would be interpreted as an increase in bacteriallyderived cell-wall material entering the DOM pool. However, this was not the case (Figure 4-17). No discernible difference existed between the protozoan cultures and the bacterial control. This is consistent with results observed by Tranvik (1994). Since the colloids in Nagata and Kirchman were thought to be derived from membranes, the LPS assay would not give us any information on this pool of material. Bulk lipid data (Table 4-5) showed that the amount of lipids in the culture filtrates was comparable to that found in the studies of Nagata and Kirchman (1992b). In contrast to Nagata and Kirchman (1992b), though, there was no evidence of enhanced phospholipid concentrations and thus, there was no evidence of the production of phospholipid-rich material in our cultures. 


\subsection{Conclusions}

The purpose of this study was to examine the temporal and compositional dynamics of "grazer-enhanced" DOM in three protozoan species cultures. Bulk DOC cycles were not tightly coupled to the sub-pools measured, and production behavior of other components of DOM such as surfactants and lipids could not be predicted from DOC concentrations. Surface activities were monitored in all cultures and its production was highest in ciliate cultures grown on yeast extract-bacteria. This production was related to high protozoan concentrations, though the timing was usually right after maximum ingestion rates. Although, lipid concentrations in protozoan cultures were consistent with previous work by Nagata and Kirchman (1992b), no direct evidence of lipid-rich colloidal material was evident in these experiments. 


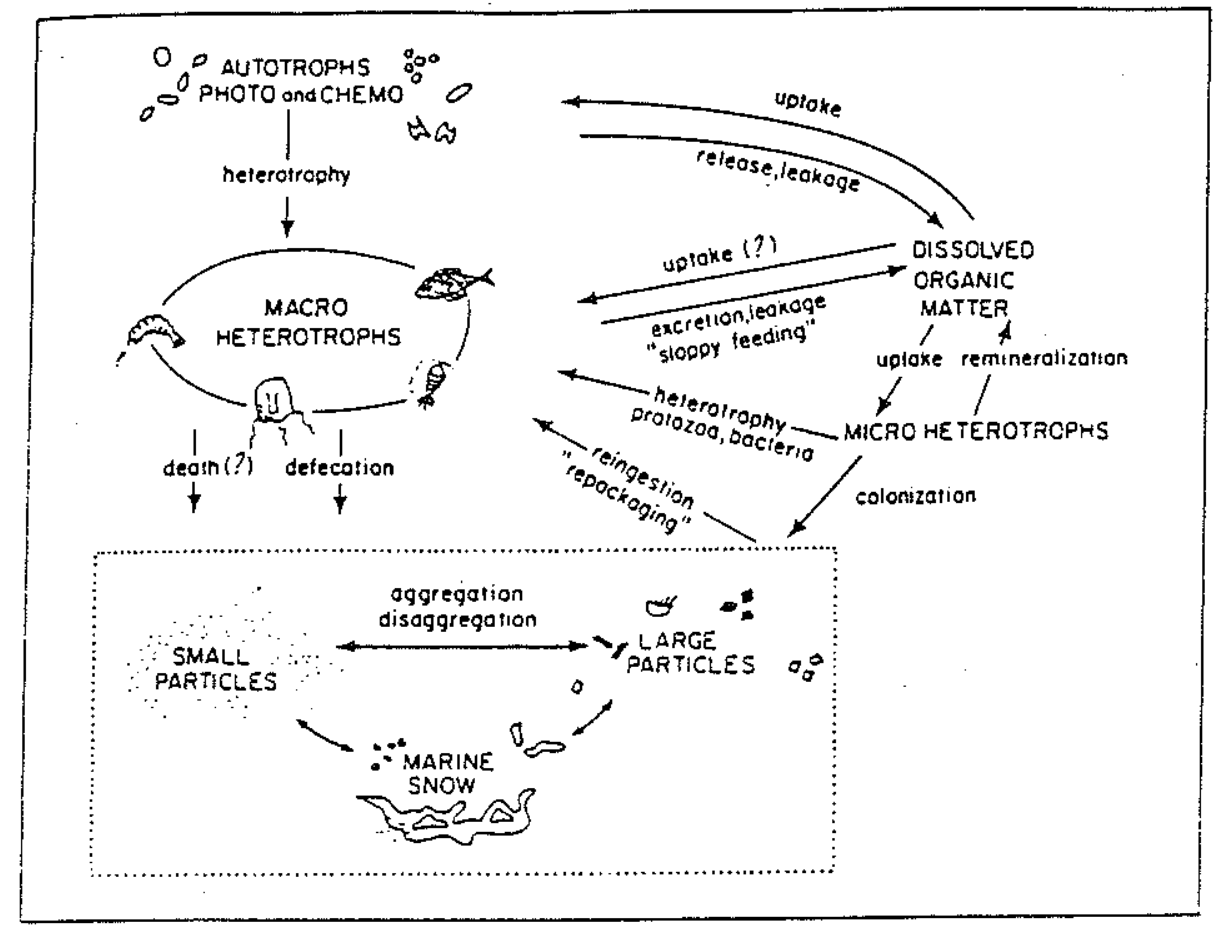

Figure 4-1. Organic carbon cycle in surface ocean as result of food web cycling Figure taken from Lee \& Wakeham (1988).

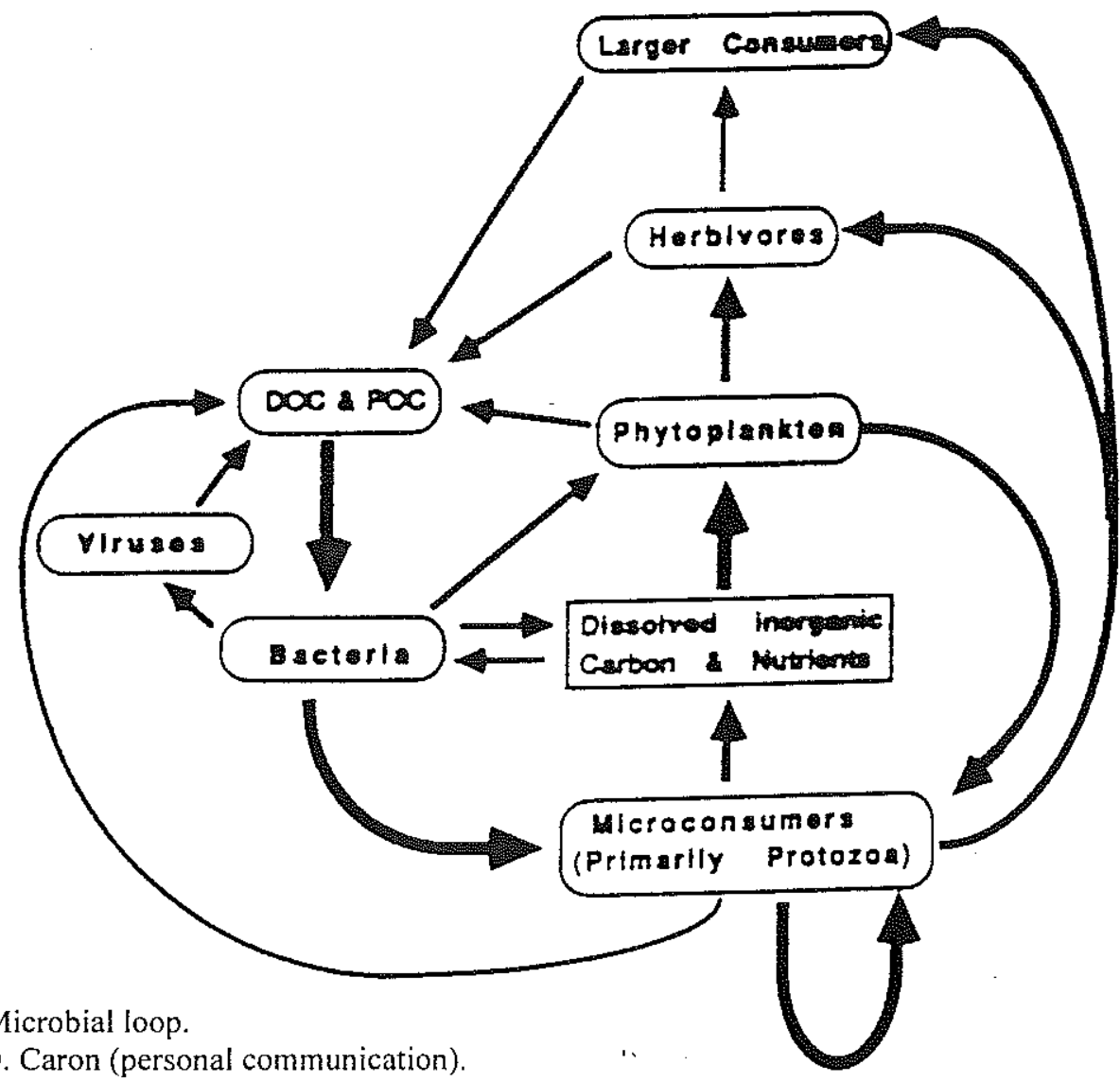

Figure 4-2. Microbial loop.

Figure from D. Caron (personal communication). 


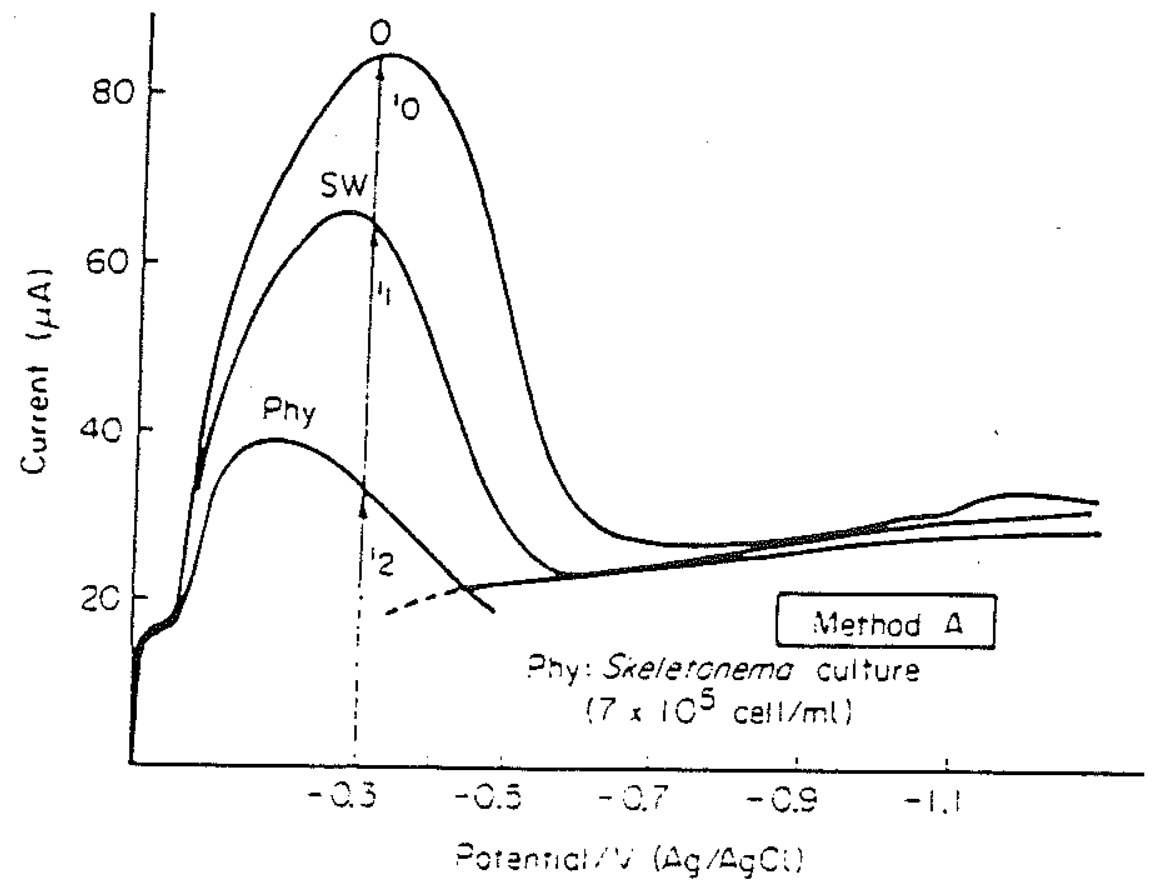

Figure 4-3. Example of results obtained by surfactant method.

Figure taken from Zutic et al. (1981). Surfactant activity is related to the decrease in peak height (current) from the operationally-defined zero (in the case of the present study, Vineyard Sound seawater). The activity is expressed in terms of the external standard used (in this case, Triton X-100). 
A

Surfactants

$\square$ Syringe $\square$ Centrifuge Vacuum

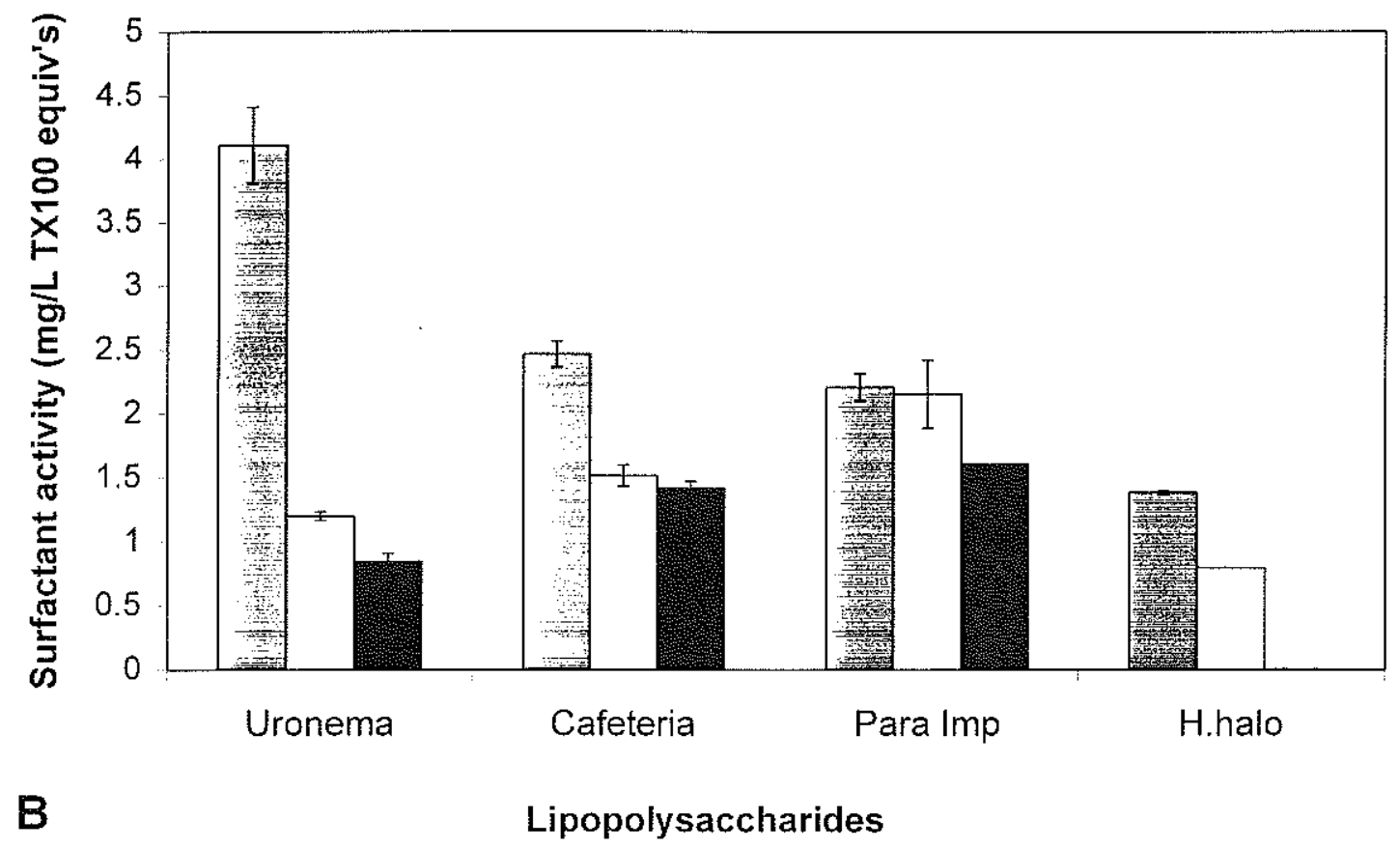

Syringe $\square$ Centrifuge Vacuum

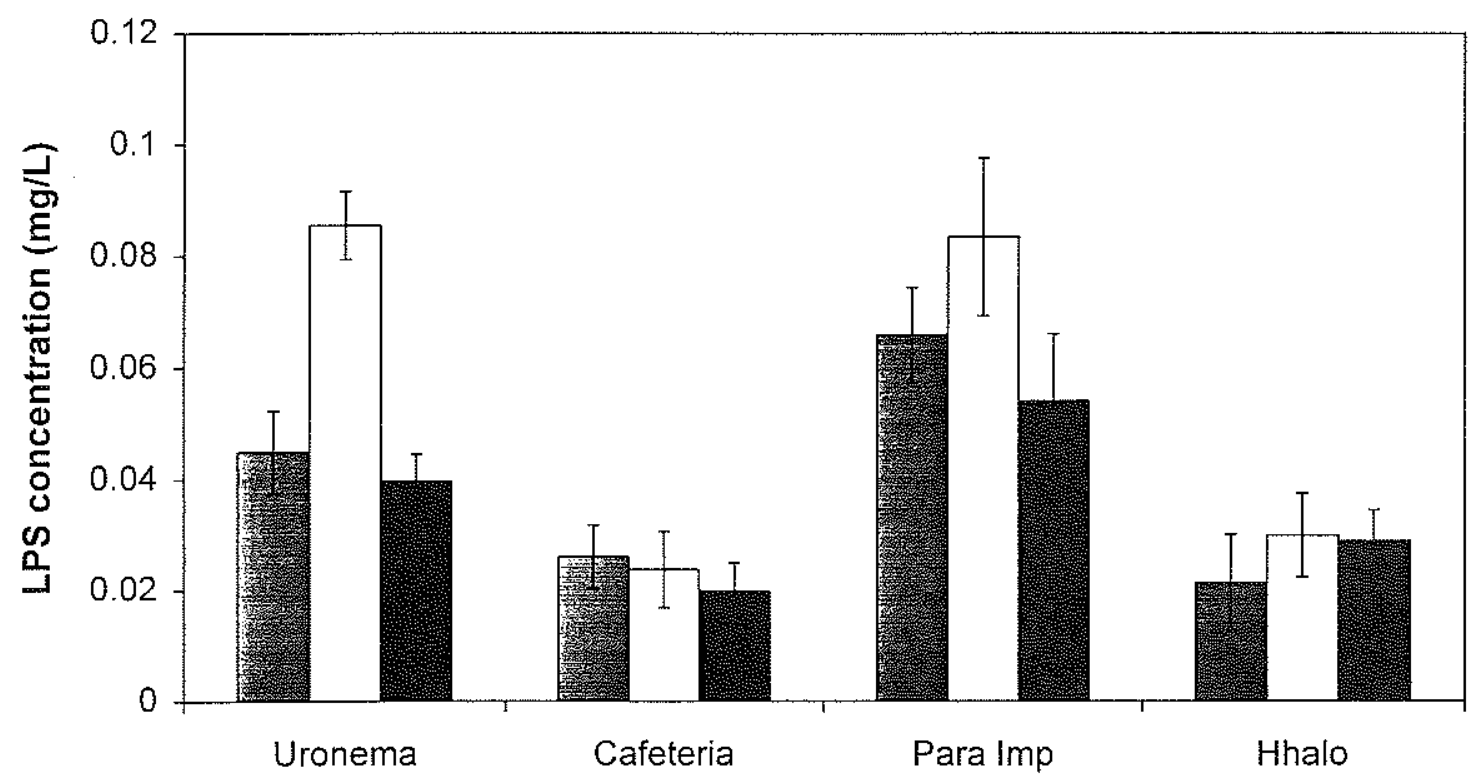




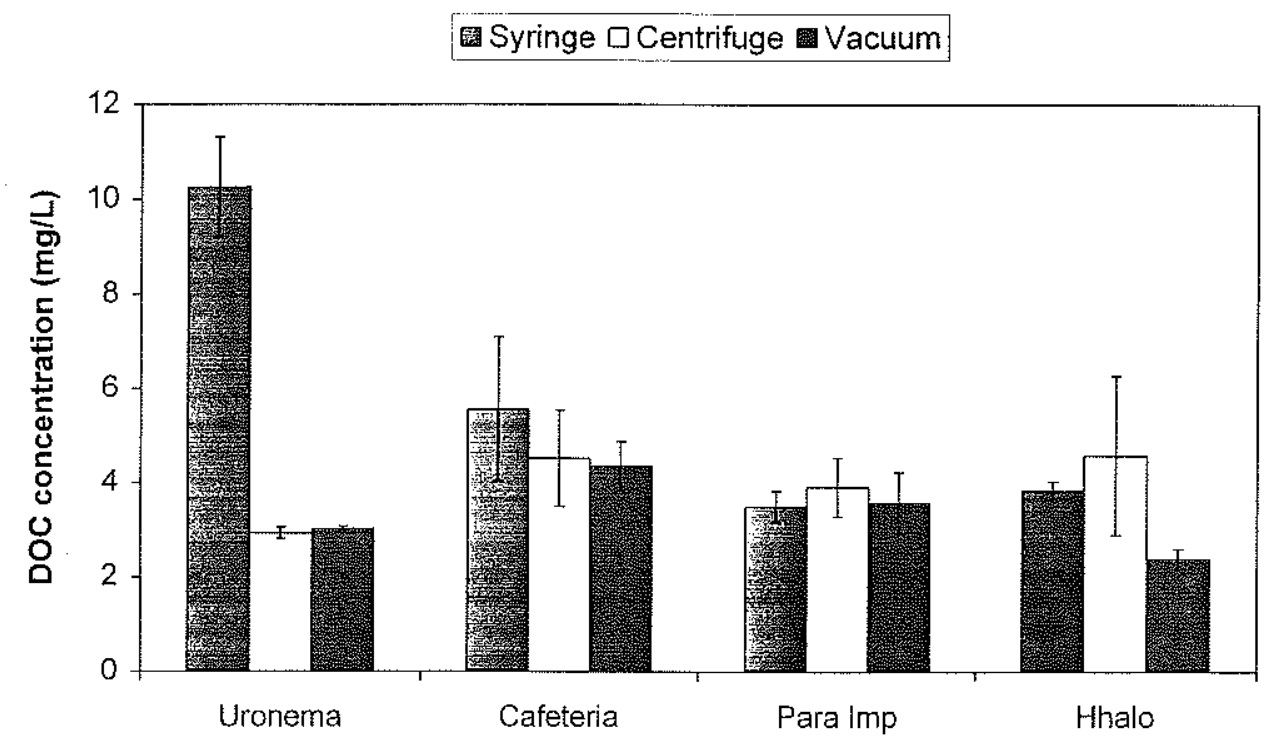

Figure 4-4. Collection method study for dissolved parameters - surfactants, LPS, and DOC.

"Dissolved" samples collected with one of three methods $-0.2 \mu \mathrm{m}$ syringe filtration (grey) through surfactant-free cellulose acetate filters, centrifugation (open/white) at $10,000 \mathrm{rpm}$ for $45 \mathrm{~min}$, and vacuum filtration (black) through $0.2 \mu \mathrm{m}$ Nuclepore polycarbonate filters. All samples were collected at end point of interspecies comparison experiment (March 1999). (A) Surfactant samples. (B) Lipopolysaccharide samples. (C) DOC samples.

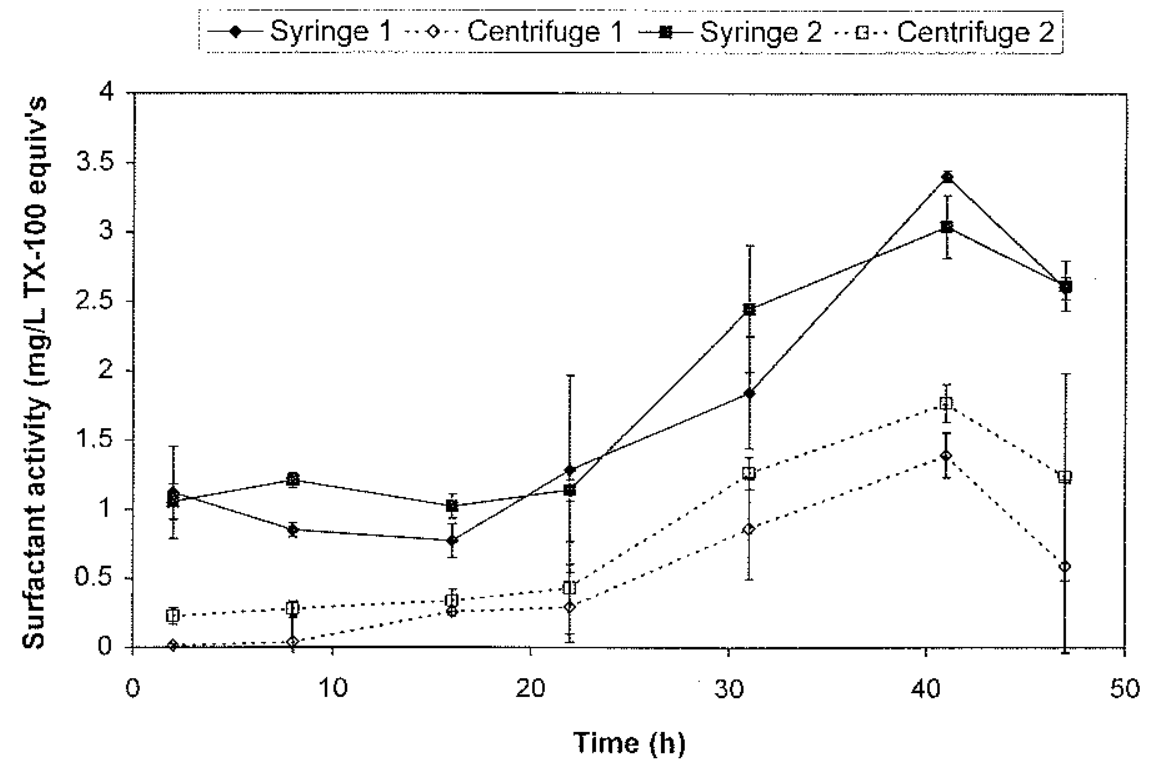

Figure 4-5. Collection study for Uronema culture - syringe filtration vs. centrifugation.

For the duration of a grazing experiment, dissolved samples were collected in replicate grazing cultures both by syringe filtration (through $0.2 \mu \mathrm{m}$ SFCA syringe filters) and by centrifugation $(10,000 \mathrm{rpm}$ for $45 \mathrm{~min}$ ). Solid lines represent syringe filtered samples (filled symbols: diamonds = replicate 1; squares = replicate 2) and dotted lines represent centrifuged samples (open symbols). In short, the temporal trends are the same regardless of collection method. 


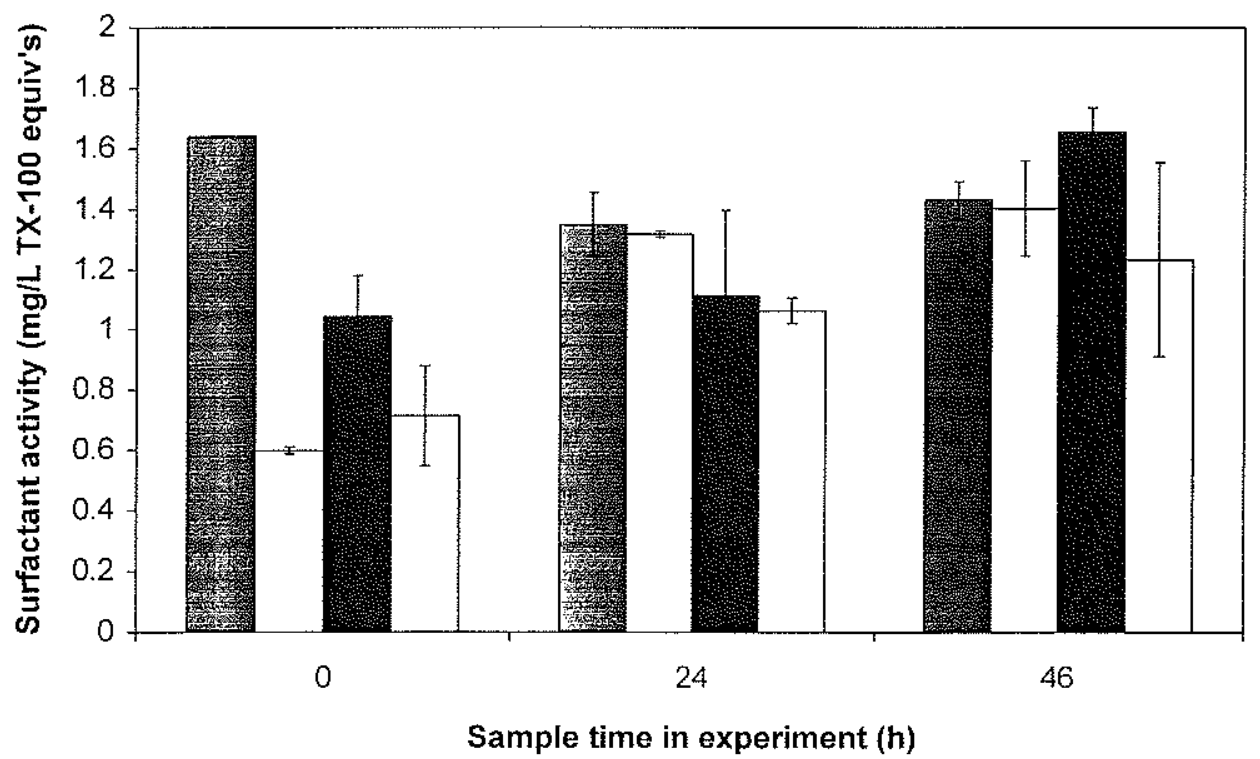

B Day 1 Day 4 Day 7 DDay 21

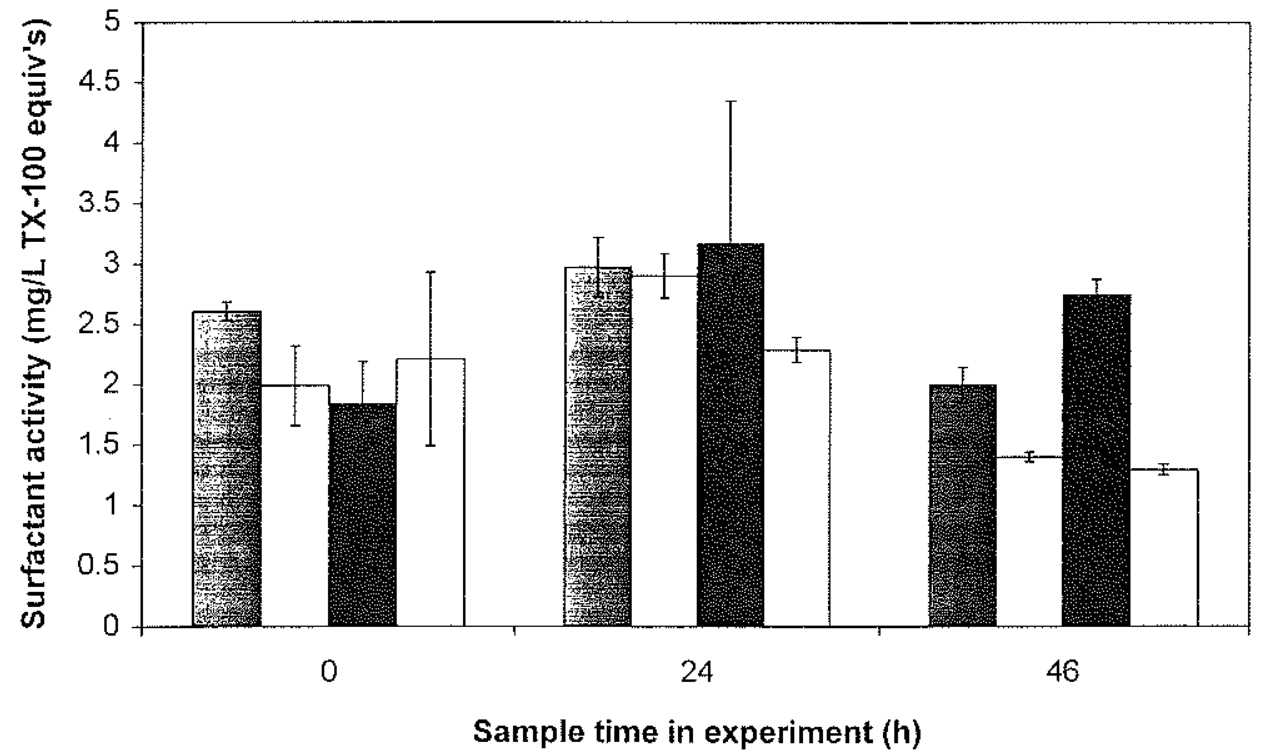

Figure 4-6. Storage experiment for H.halodurans and Uronema surfactant samples.

All samples for this storage experiment were collected via $0.2 \mu \mathrm{m}$ syringe filtration (SFCA filters) during a protozoan grazing experiment. Samples were collected at three different time points within the experiment $(0 \mathrm{~h}, 24 \mathrm{~h}$, and $46 \mathrm{~h})$. Samples were then stored in a $4^{\circ} \mathrm{C}$ refrigerator until analysis on Day 1 (column1 / grey), Day 4 (column2 / white), Day 7 (column3/ black) and Day 21 (column4 / light grey). On an analysis day, two samples from each experimental time point was analyzed. The graphs indicate the average and one standard deviation of triplicate analyses of duplicate samples. A: Samples collected from the bacterial (H.halodurans) control experiment. B: Samples collected from the ciliate culture. 
Day $0 \square$ Day 16 Day 30

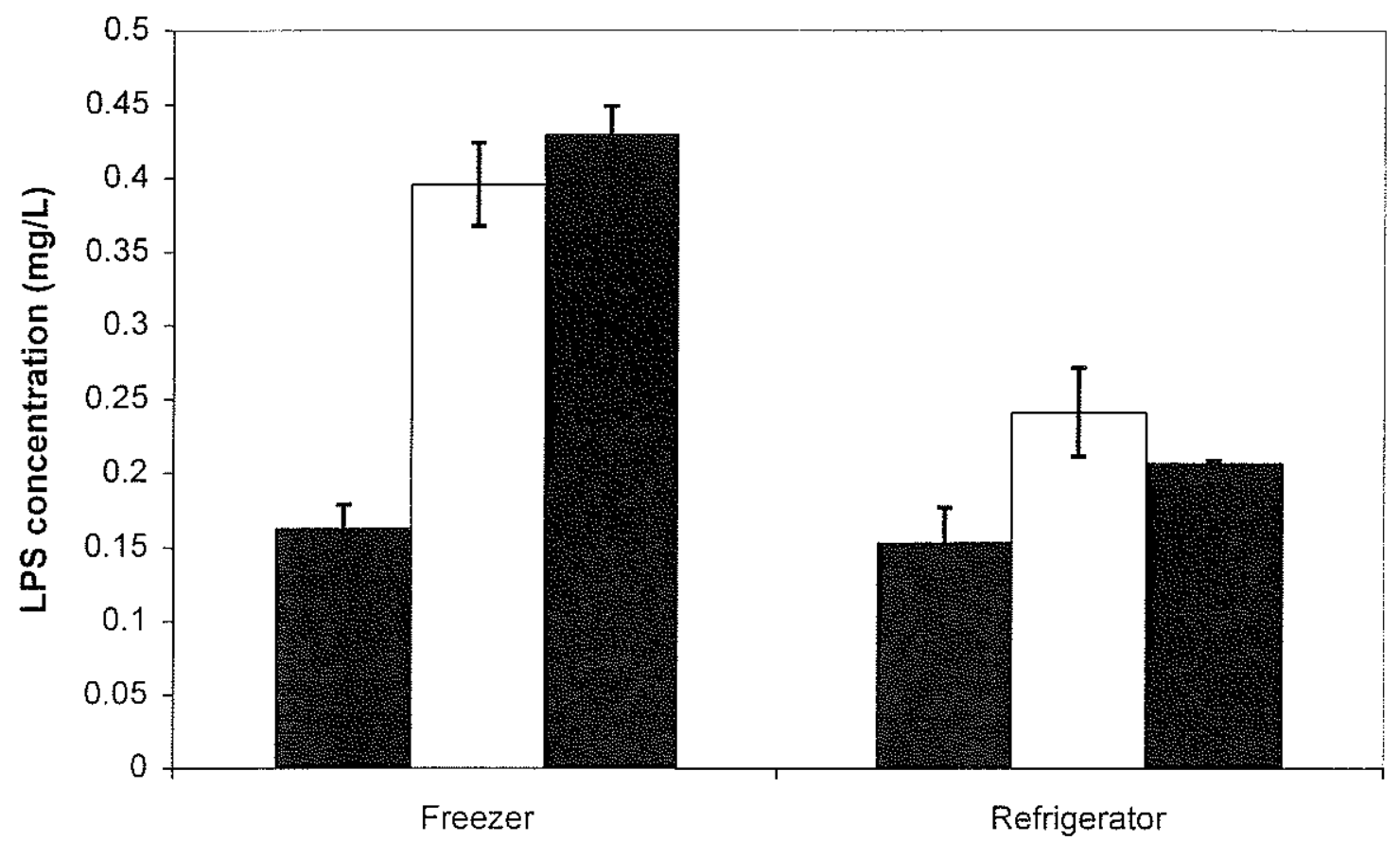

Figure 4-7. Lipopolysaccharide storage experiment for H.halodurans culture.

Samples were collected from a H.halodurans control culture through $0.2 \mu \mathrm{m}$ SFCA syringe filters. Samples were analyzed immediately after collection (Day $0=$ grey bars) and then stored either in a $-4^{\circ} \mathrm{C}$ freezer or in a $4^{\circ} \mathrm{C}$ refrigerator for 16 (open bars) and 30 (black bars) days. Each bar represents the average of two samples run in triplicate. 

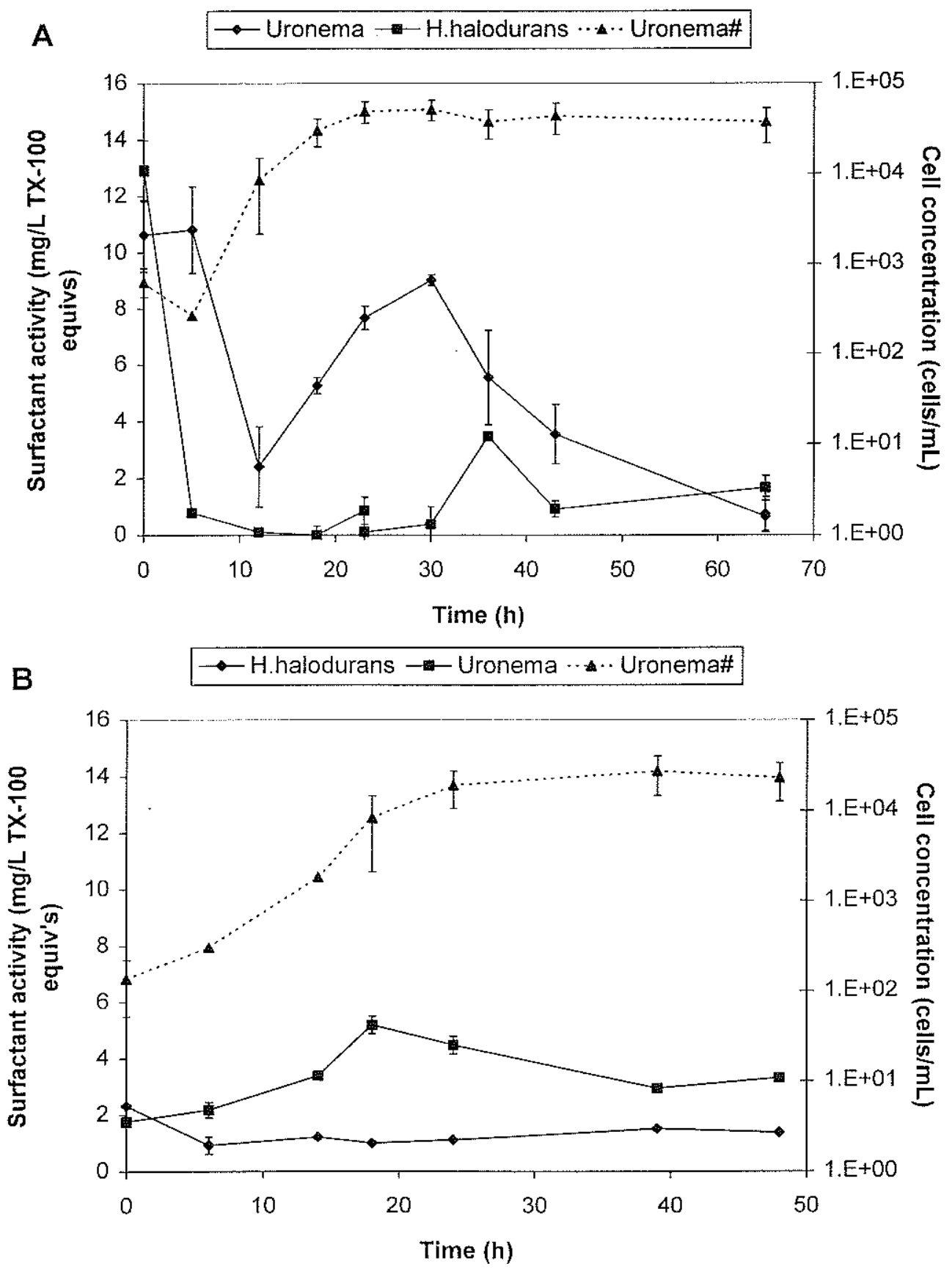

Figure 4-8. Surfactant concentrations and Uronema \# versus time in two early experiments.

Surfactant concentrations were monitored over time in a Uronema grazing culture (squares) as well as a bacterial control ( $H$. halodurans - diamonds). Each point is the average of triplicate analyses of duplicate samples $\pm 1 \sigma$. For comparative purposes, the Uronema numbers are shown as well. The bacteria in both experiments were grown on Yeast Extract media. The rapid initial decrease in surfactant concentration in the first experiment $(\mathbf{A})$ is potentially due to bacterial utilization. The onset of surfactant production is coincident with protozoan exponential growth. The second experiment (B) exhibited similar behavior, except that both the maximum surfactant concentration and protozoan number are lower than the first. 


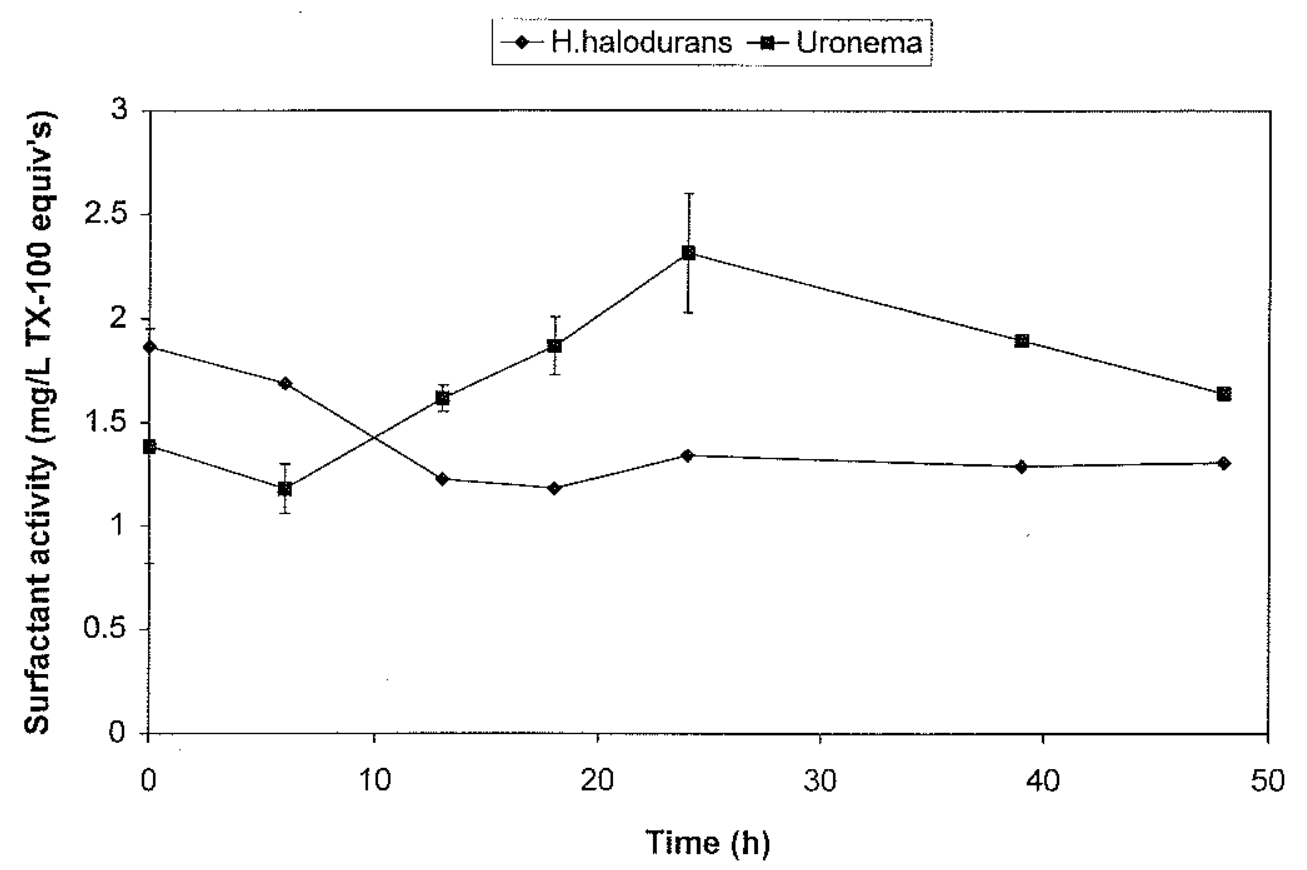

Figure 4-9. Surfactant concentrations in a Uronema culture with glucose-grown prey.

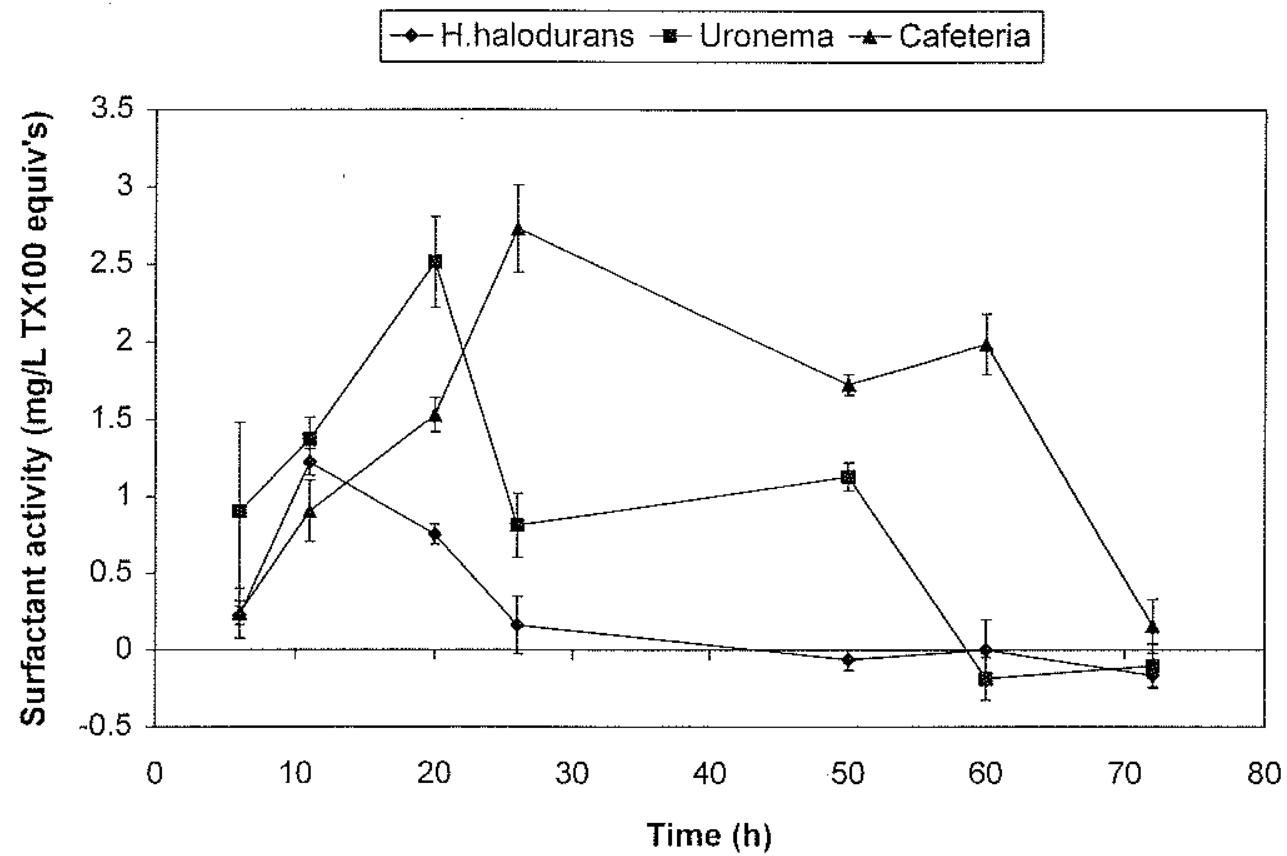

Figure 4-10. Surfactant concentrations in a Uronema and a Cafeteria culture with pyruvate-grown prey. Samples were collected for this experiment with $0.2 \mu \mathrm{m}$ cellulose acetate syringe filters. These filters have a consistent bleed that was subtracted from each sample. Thus, some of the samples are below zero but the error bars show that the samples are not statistically different from zero. Bacterial control: diamonds; Uronema: squares; Cafeteria: triangles. 

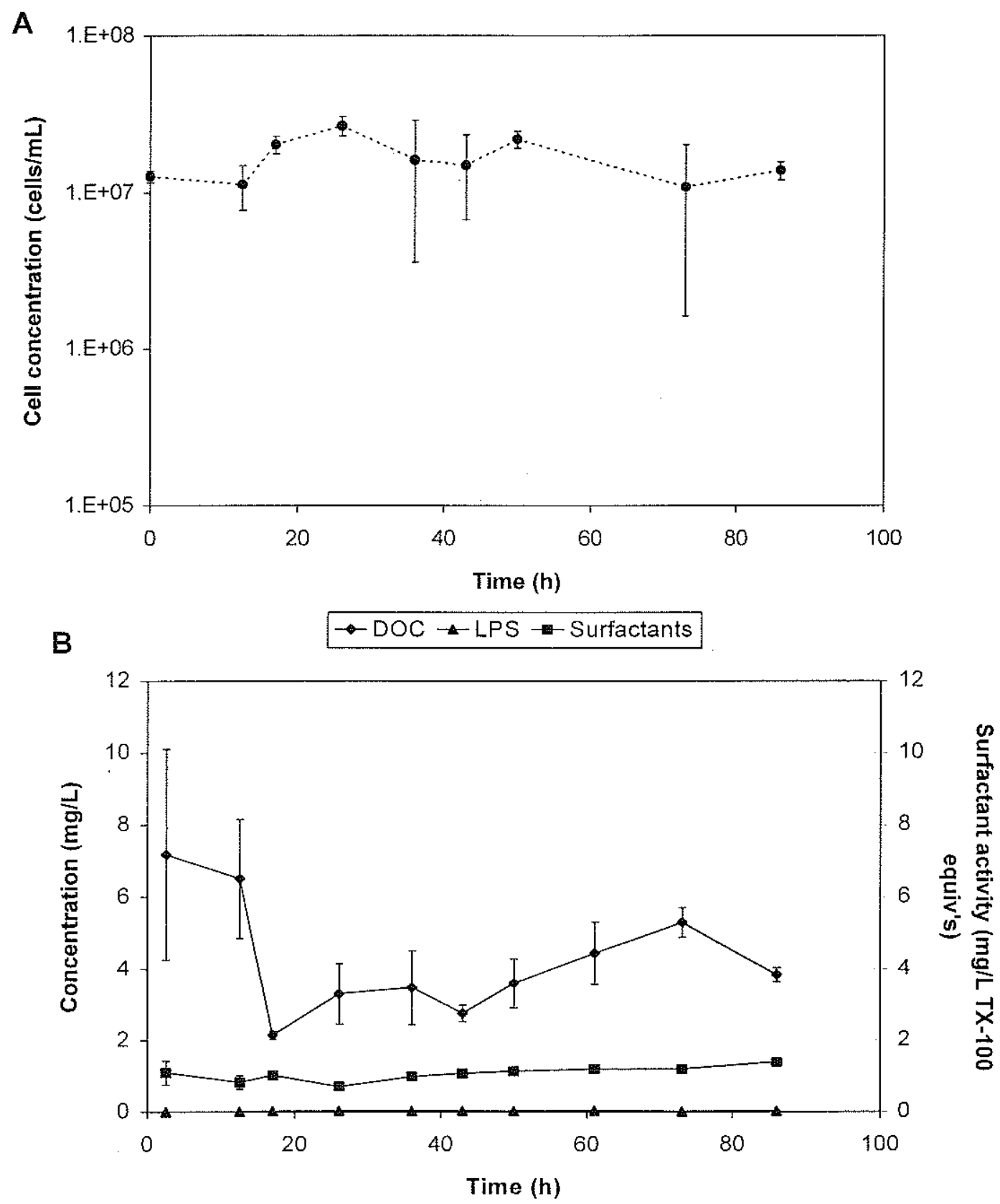

Figure 4-11. Data for interspecies comparison - Hhalodurans - population, DOC, LPS, and surfactants. Bacteria numbers (A) were determined using epifluorescence microscopy of AO-stained samples.

Dissolved samples (B) were collected via $0.2 \mu \mathrm{m}$ SFCA syringe filtration. DOC samples (diamonds) are the average of triplicate analyses $\pm 1 \sigma$, surfactant concentrations (squares) are the average of triplicate analyses of duplicate samples $\pm 1 \sigma$, and LPS concentrations (triangles) are the average of triplicate analyses $\pm 1 \sigma$. Some error bars are smaller than the size of the symbols. 

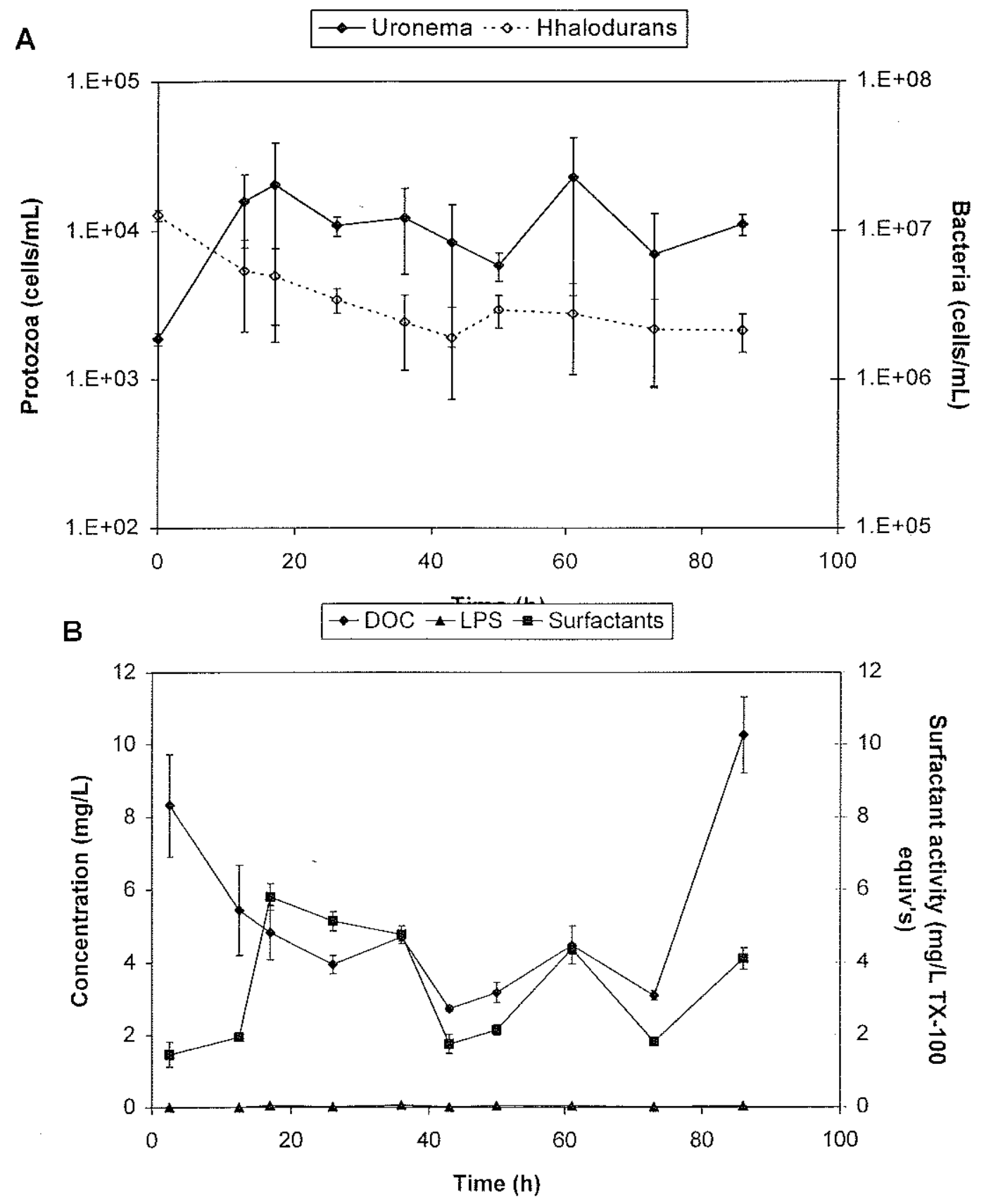

Figure 4-12. Data for interspecies comparison-Uronema - populations, DOC, LPS, and surfactants. Data prepared in same manner as Figure 4-11. In the population graph (A), protist numbers are represented by diamonds with a solid line and bacteria numbers are represented by open diamonds with a dashed line. Dissolved parameters (B) were collected via syringe filtration. Again, LPS concentrations are extremely low relative to DOC and surfactant concentrations. 

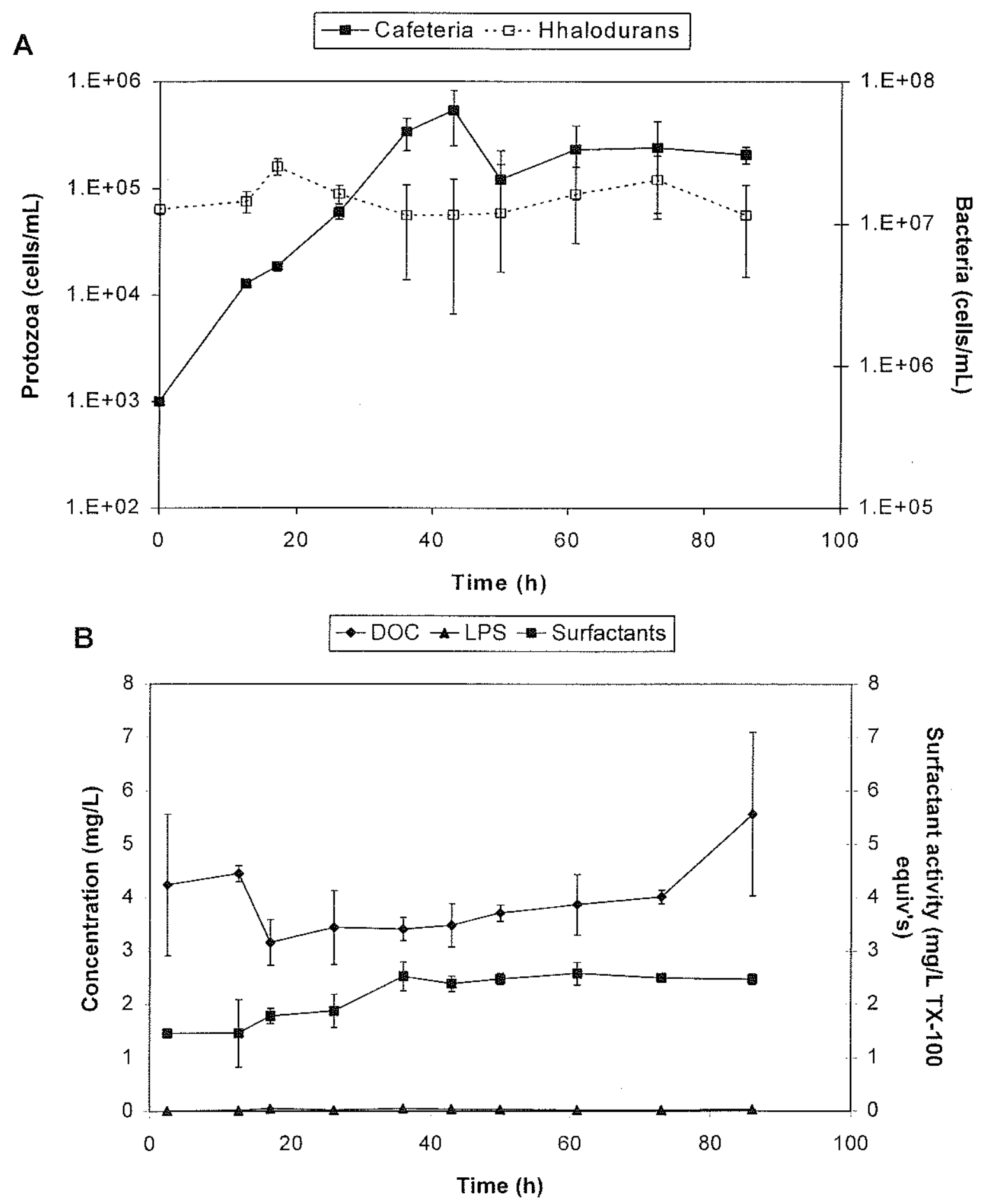

Figure 4-13. Data for interspecies comparison - Cafeteria - population, DOC, LPS, and surfactants. Data prepared in same manner as Figures 4-11 and 4-12. 

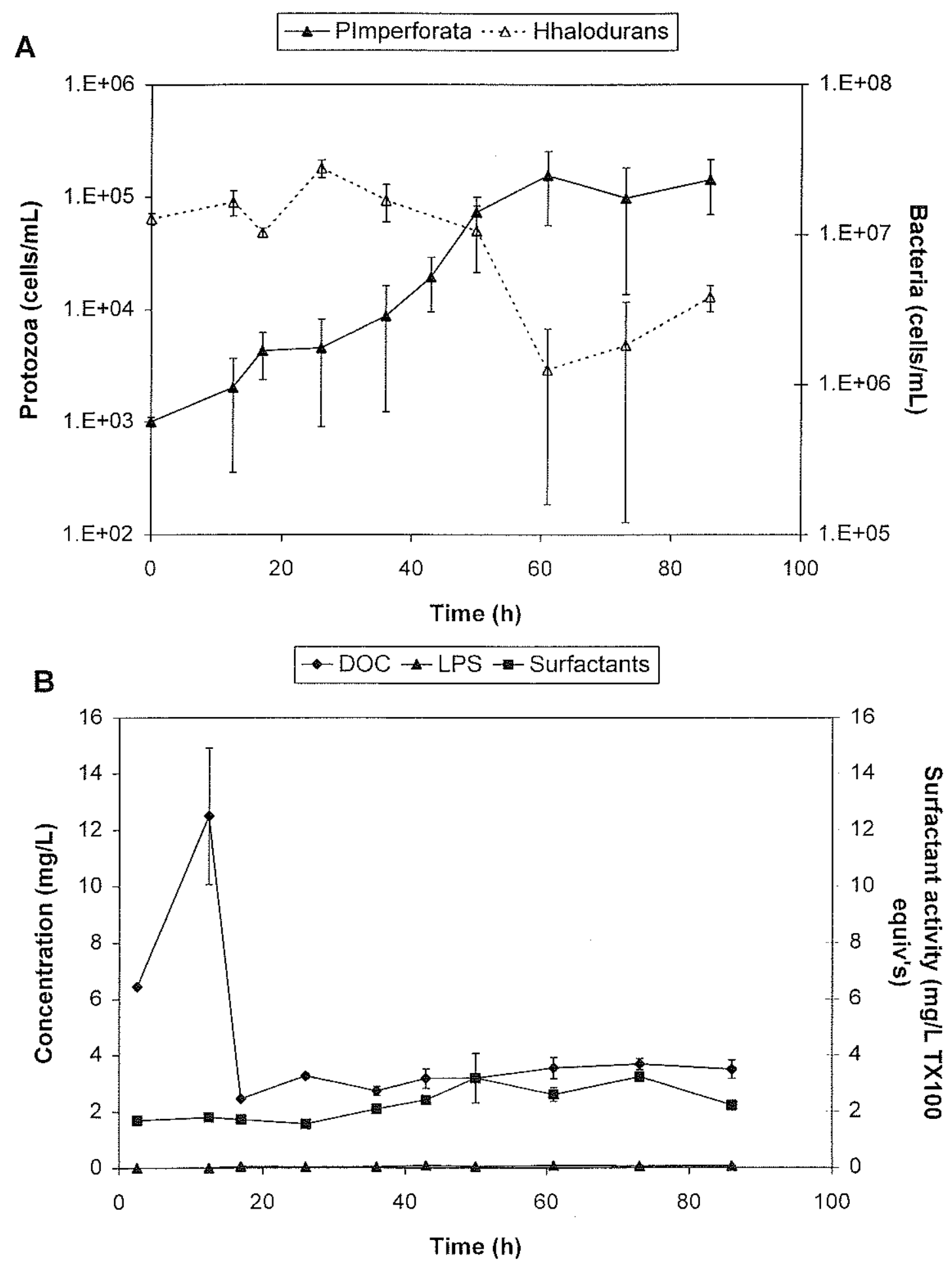

Figure 4-14. Data for interspecies comparison - $P$. Imperforata - population, DOC, LPS, and surfactants. Data prepared in same manner as Figures 4-11 and 4-12. 


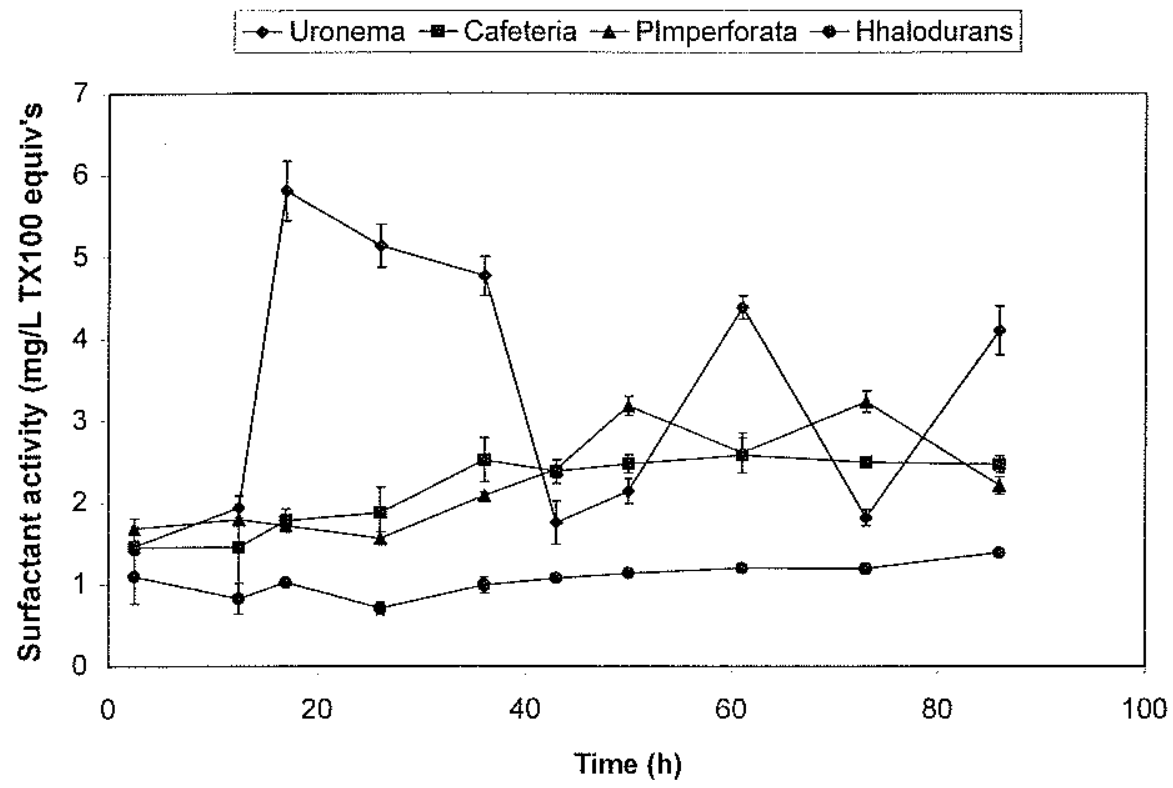

Figure 4-15. Surfactant data for all cultures in interspecies comparison.

Surfactant concentrations from all cultures are compared: Uronema (diamonds), Cafeteria (squares), Paraphysomonas imperforata (triangles), and $H$. halodurans (circles). The bacterial control remains low throughout the experiment while large fluctuations are observed in the Uronema cultures. A gradual increase in surfactant concentrations is observed in both flagellate cultures.

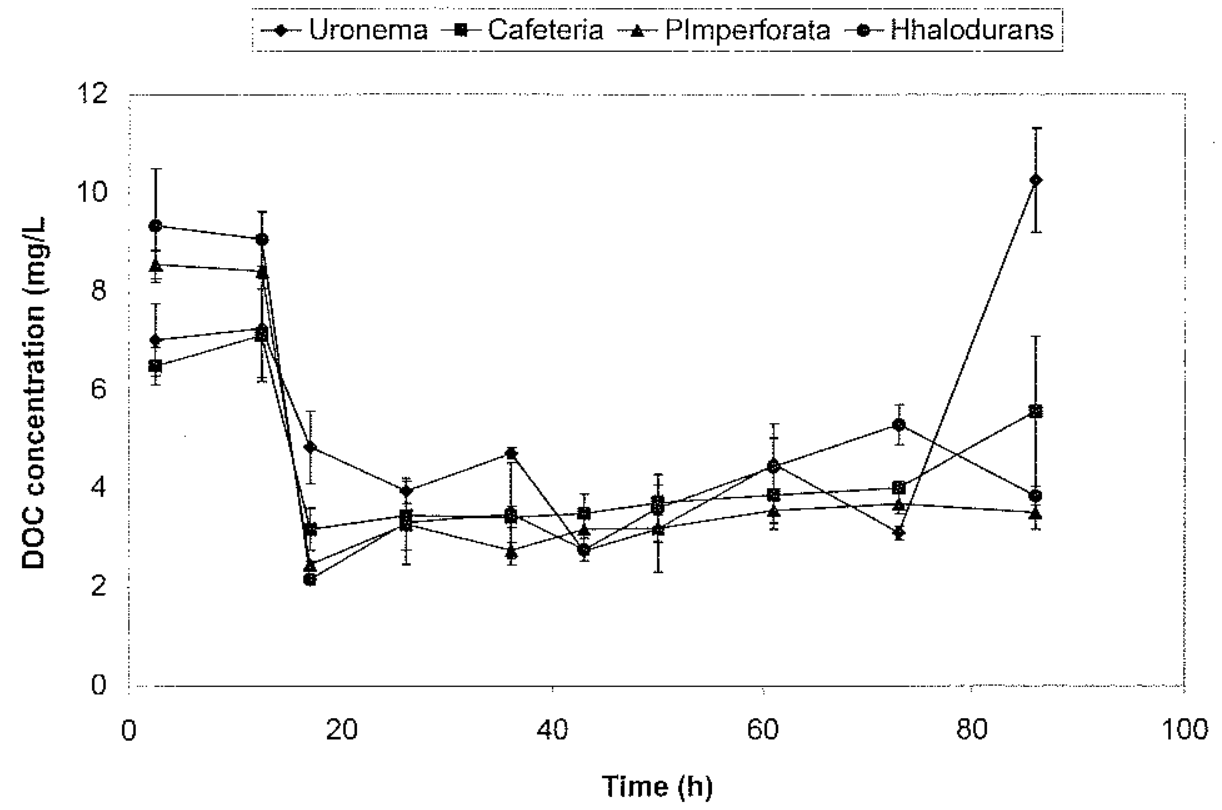

Figure 4-16. DOC data for all cultures in interspecies comparison.

DOC concentrations from all cultures: Uronema (diamonds), Cafeteria (squares), P.imperforata (triangles), and Hhalodurans (circles). The cultures are indistinguishable from one another. Initial decrease is potentially due to bacterial utilization. 


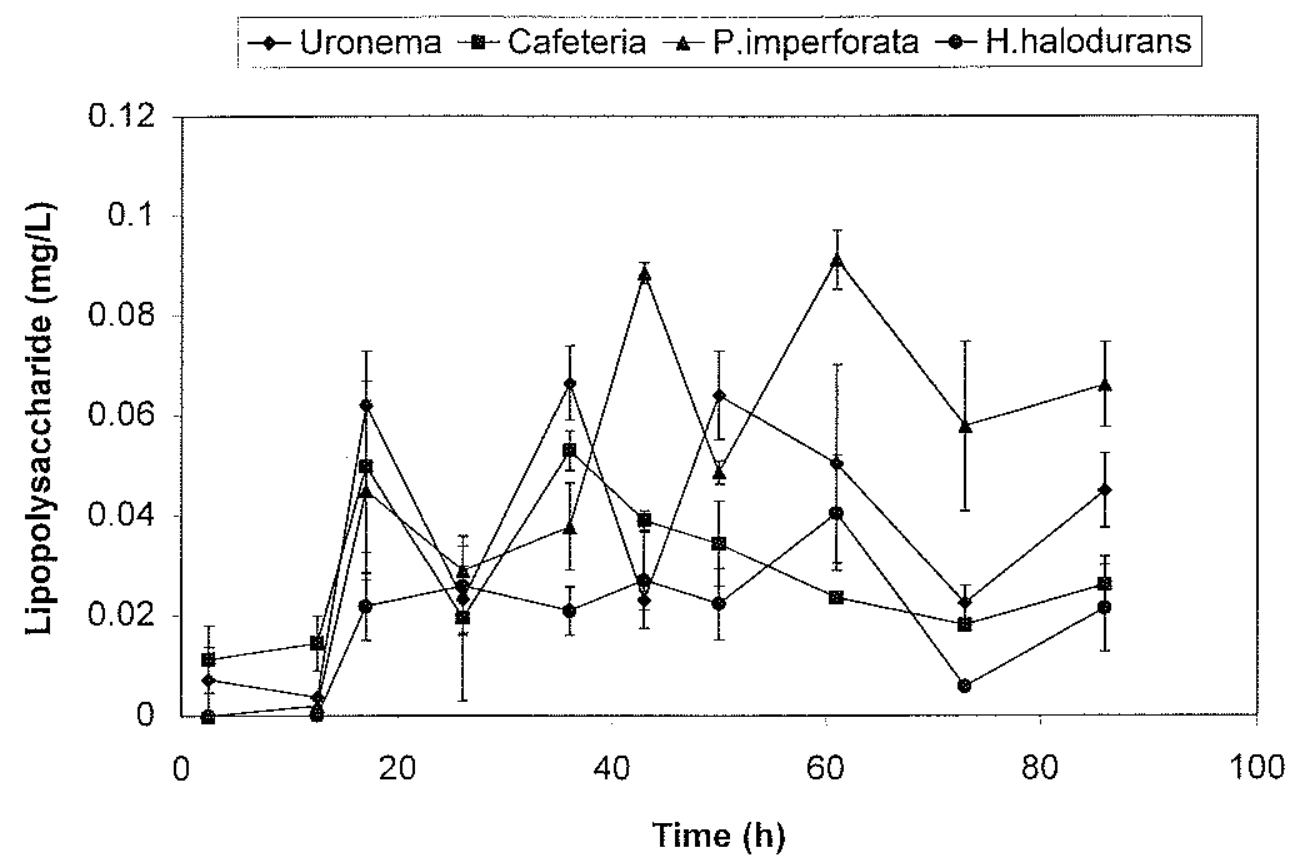

Figure 4-17. LPS data for all cultures in interspecies comparison.

LPS concentrations for all organisms are shown: Uronema (diamonds), Cafeteria (squares), P.Imperforata (triangles), and Hhalodurans (circles).

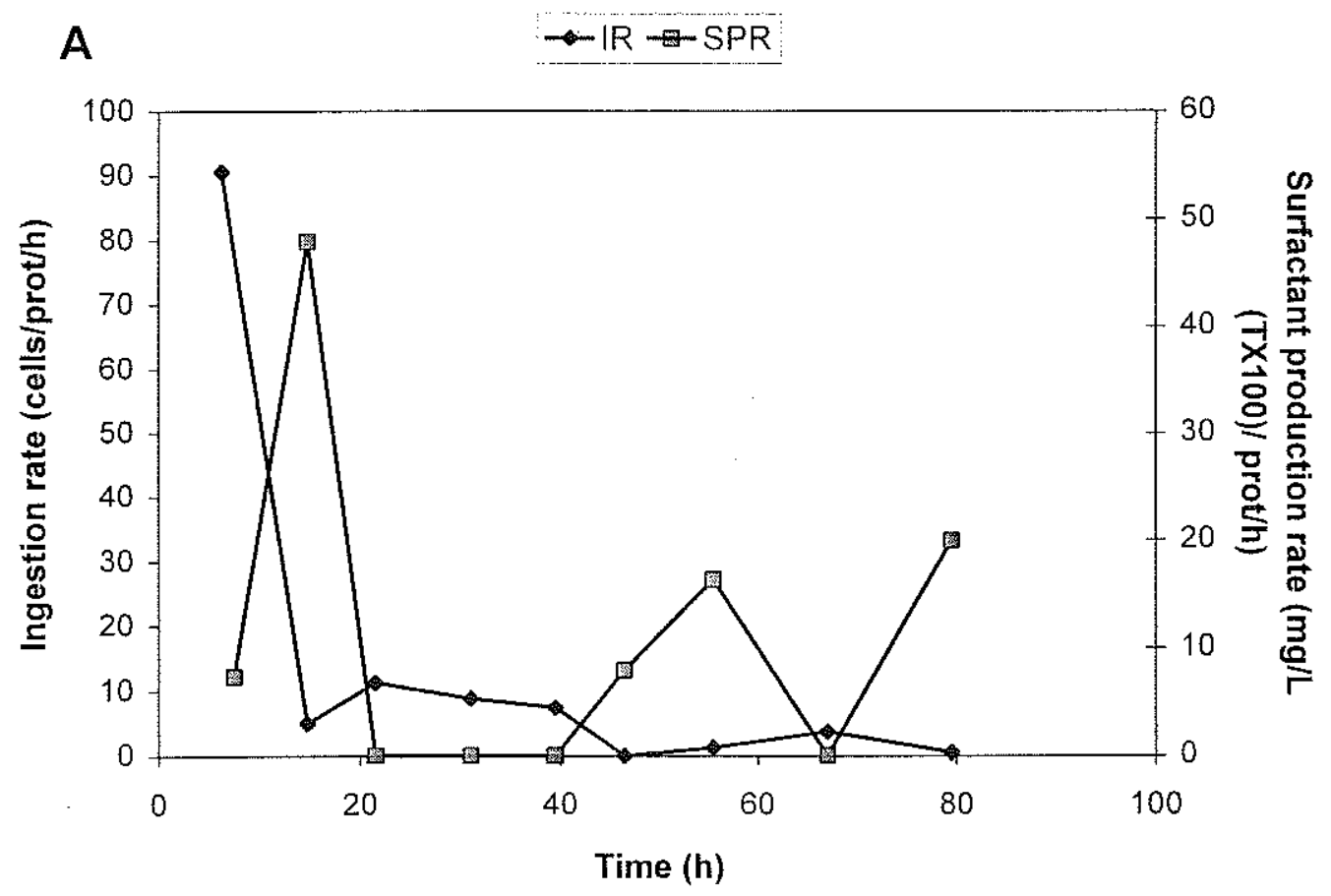



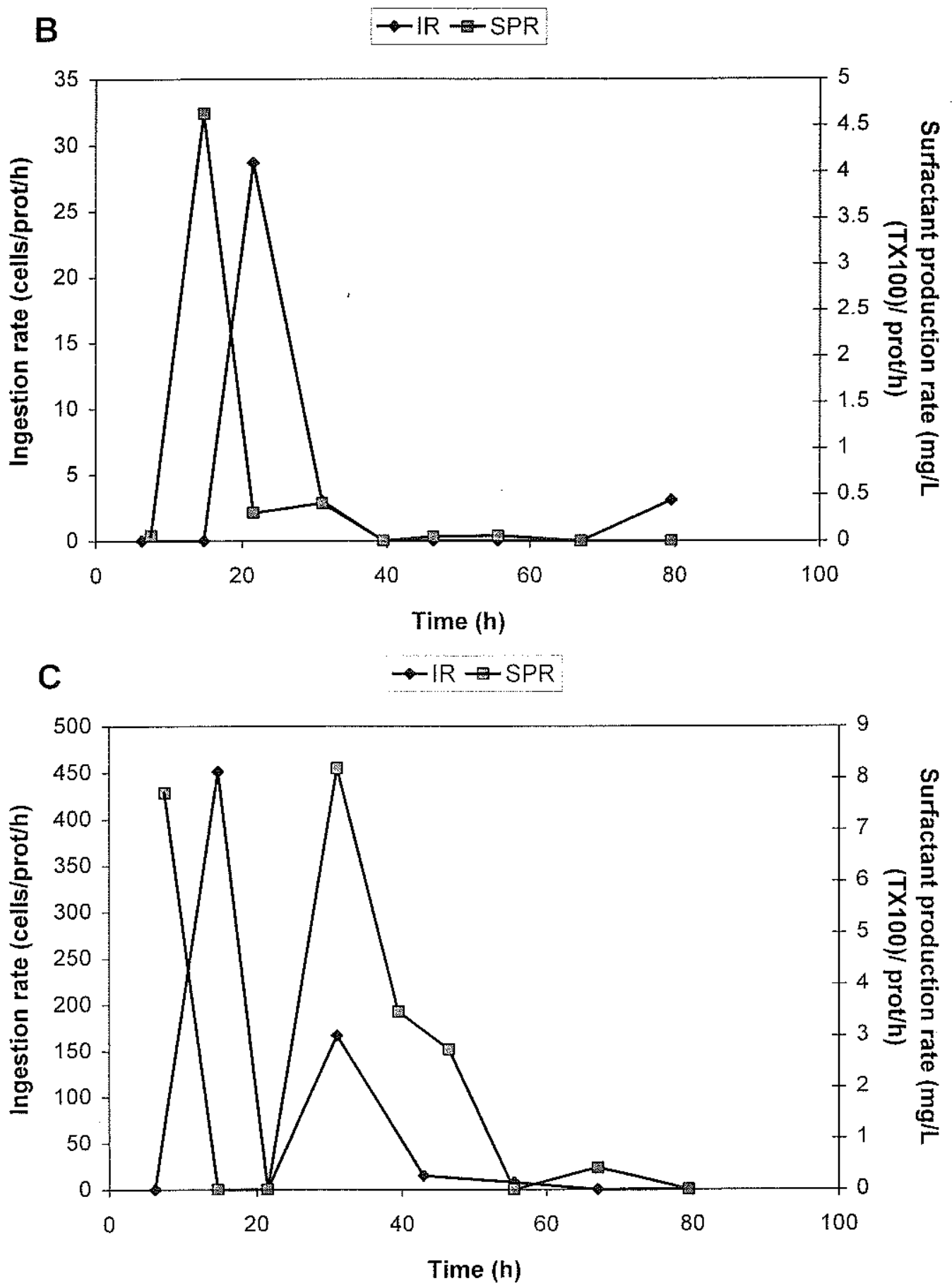

Figure 4-18. Comparison of surfactant production rates and ingestion rates in 3 protozoan species. In each figure, the diamonds represent the ingestion rate (as calculated using Equation 1) and the squares represent the surfactant production rate (as calculated using Equation 2). Figure A: Uronema culture; Figure B: Cafeteria culture and Figure C: P. imperforata culture. 


\section{Effect of DOC components on $C B$ speciation in protozoan culture filtrates}

\subsection{Introduction}

\subsubsection{CB speciation in natural waters - "bioavailable" fraction}

Polychlorinated biphenyls (PCBs) are distributed among three different phases in aquatic systems - truly dissolved, associated with colloidal or dissolved organic material (C/DOM), and associated with particulate material (both organic and inorganic phases) (Farrington and Westall, 1986; Duursma et al., 1989; Chin and Gschwend, 1992; McGroddy and Farrington, 1995). Several studies have shown that the pool most important for accumulation in aquatic food webs is the truly dissolved fraction (review Farrington, 1991). An understanding of the dynamics and physico-chemical parameters influencing this pool of PCBs is crucial to estimating the impact of PCBs on the health of organisms within an ecosystem.

PCBs are hydrophobic and lipophilic compounds and therefore accumulate in organic- or lipid-rich material. Equilibrium partition coefficients (defined as $\mathrm{K}_{\mathrm{org}}=$ $\mathrm{C}_{\mathrm{org}} / \mathrm{C}_{\mathrm{w}}$ - where $\mathrm{C}_{\mathrm{org}}$ is the concentration of PCBs in the organic phase (mass PCBs per $\mathrm{g}$ organic phase) and $\mathrm{C}_{\mathrm{W}}$ is the concentration in the aqueous phase (mass PCBs per $\mathrm{mL}$ solution)) have been used to compare the relative tendencies of different hydrophobic compounds to accumulate in organic phases. $N$-octanol is used as the reference organic phase and the resulting partition coefficient is referred to as $\mathrm{K}_{\mathrm{ow}}$. These partition coefficients range from $10^{4}$ to $10^{8}$ for the 209 congeners within the chlorobiphenyl (CB) compound class. The values of $\mathrm{K}_{\mathrm{ow}}$ can be related to environmentally relevant organic phases such as lipids and soil organic matter by linear free energy relationships as shown in Schwarzenbach et al. (1993). Lipid material is very similar to $n$-octanol and so the partition coefficient, $\mathrm{K}_{\mathrm{iw}}$, is almost equivalent to $\mathrm{K}_{\mathrm{ow}}$. Soil organic matter is not as good a sorbent for PCBs as lipid material and so, the partition coefficient, $\mathrm{K}_{\mathrm{oc}}$, tends to be approximately one order of magnitude lower than $\mathrm{K}_{\text {ow }}$ (Schwarzenbach et al., 1993). 
Initial studies regarding the aqueous/particulate behavior of hydrophobic organic contaminants assumed that sediment/water systems were biphasic (Karickhoff et al., 1979). Subsequent investigations noted enhanced aqueous concentrations over that expected from aqueous solubility calculations (Carter and Suffet, 1982; Chiou et al., 1986). These enhanced aqueous concentrations have since been attributed to interactions between hydrophobic contaminants and colloidal or dissolved organic matter (Gschwend and Wu, 1985; Baker et al., 1986; Brownawell and Farrington, 1986; Gunnarsson and Rosenberg, 1996). Since the recognition of the role of colloidal/dissolved organic matter in contaminant speciation, numerous studies have attempted to collect and characterize this material and its sorptive qualities (Schlautman and Morgan, 1993; Chin et al., 1997; Gustafsson and Gschwend, 1997). Sources and sinks of this material, its composition and size/ structure, and its residence time in the water column are still poorly characterized.

Traditionally, DOC was assumed to be a large geopolymer with aromatic and acid functional groups (e.g., Gagosian and Stuermer, 1977). This paradigm was based on studies of humic-like and fulvic-like compounds concentrated on resins. This material represented only $5-10 \%$ of total DOC. With the advent of ultrafiltration and other concentration techniques, $30-40 \%$ of DOC could be concentrated and characterized. Recent work by Aluwihare et al. (1997) showed that DOC in the $>10,000 \mathrm{D}$ (nominal) size range was predominantly complex polysaccharide, or carbohydrate. The material concentrated and analyzed by Aluwihare et al. (1997) appeared to be of recent biological origin, rather than the product of geo-polymerization reactions. Further culture studies by Aluwihare and Repeta (1999) showed that phytoplankton exudates could be degraded by bacterial assemblages to material with similar spectral (also presumably structural) properties to marine HMW DOM.

HMW DOM is presumed to originate from primary production and is lost from the system in question by either degradation by secondary users (such as bacteria) or aggregation of colloids and subsequent settling of small particles. The residence time of DOM is governed by the balance between production and loss mechanisms. The composition and/or structure of the organic matter play a large role in its ability to 
associate with and sorb PCBs. Materials with high carbohydrate content tend to be less sorbent than materials with high percentages of lipid material (Garbarini and Lion, 1986). PCBs will move from the aqueous phase into colloidal aggregates or dissolved macromolecules only if a microenvironment can be formed within the folds and aggregates of this material (Gustafsson and Gschwend, 1997). This microenvironment must be less polar than water and large enough to at least partly accommodate the contaminant. Some studies have shown decreasing sorption with increasing organic phase polarity (decreasing C/O ratios - Chiou et al., 1986; Gauthier et al., 1987). However, in general, sorption to colloidal organic matter is similar to the sorption to bulk organic matter within a system (Schwarzenbach et al., 1993).

There are a myriad of terms used to describe the association between organic contaminants such as PCBs and a natural organic phase or sorbent. The interaction between PCBs and organic matter is not a covalent chemical bond but instead is a combination of van der Waals, dipole-dipole, and other molecular interactions. The general term, sorption, is used to describe the association of a CB congener with a two- or three-dimensional surface. Adsorption is a surface interaction (i.e., 2-D) and a $b$ sorption is similar to internalization (i.e., 3-D). Another distinction often made is "bound" versus "unbound". The term "bound" implies the presence of a chemical bond, a stronger interaction than sorption. In the remainder of this chapter, I'll be using the term "affinity" to describe the ability of an organic phase to sorb PCBs. "Affinity" is an intrinsic property of the material under study and can be used to compare organic material in different samples.

\subsubsection{Potential role of microbial loop (and protozoa) in CB speciation}

Work in the previous chapter and other studies (Caron et al., 1985; Taylor et al., 1985; Nagata and Kirchman, 1992b; Nagata and Kirchman, 1992a; Tranvik, 1994) showed that protozoa (and more generally, organisms within the microbial loop) were a potential source of dissolved and colloidal organic material in natural systems. Production of dissolved material by grazers is likely to be important for CB speciation in 
microbial loop-dominated systems such as the sediment-water interface of some coastal environments and oligotrophic regimes such as the Sargasso Sea. Protozoa affected both the size spectrum and the composition of particulate material in these systems by ingesting bacteria and large colloids and excreting both dissolved and colloidal material (e.g., organic: Tranvik (1994); inorganic: Barbeau and Moffett (1998)). The composition of grazer-enhanced material has been difficult to ascertain, though some studies have suggested that it is lipid-rich (Nagata and Kirchman, 1992b) while others have shown that "grazer-enhanced" DOM was dominated by bacterial internal cellular components (Tranvik, 1994).

Recent field evidence has pointed to the importance of recycling processes in the remobilization of PCBs from sinking particulate material. In particular, a recent study in Lake Superior by Baker et al. (1991) showed preferential remobilization of PCBs at the sediment-water interface. Over a particular period of time, they measured the atmospheric deposition of PCBs, PCBs associated with suspended and sinking particles, and PCBs buried in the sediments. They noted that $96 \%$ of the PCBs were caught in sediment traps and associated with sinking particles. However, $<1 \%$ of the PCBs deposited were being buried in the sediments. $90 \%$ of the organic carbon was recycled at this site, but $99 \%$ of the CBs were remobilized (see Chapter 1, Figure 1-5). Therefore, the PCBs were not following the organic carbon particulate pool as expected. Somehow, the PCBs were being returned to the water column - either by production of dissolved organic material with a high affinity for PCBs or by production of lipid-poor particulate material that has a lower affinity for PCBs. The study was repeated by Sanders et al. (1996) in a lake in England. In their sediment traps, they noted that PAHs were not preferentially remobilized at the sediment water interface as were the PCBs. They also observed that remobilization of PCBs was a function of aqueous solubility. Sanders et al. (1996) concluded that PCBs could exchange easily among pools of organic carbon whereas PAHs were sequestered in a non-exchangeable, or slowly exchanging, pool such as soot. 
Upon perusal of the Baker et al. (1991) and Sanders et al. (1996) studies, it was clear that chemical and structural changes occurring within the organic material was affecting the fate and transport of PCBs within this system. These compositional changes could be altering the affinity of bulk DOM for PCBs. The microbial loop is efficient in remineralizing organic material and it is possible that microbial-mediated processes are responsible for the preferential remobilization of PCBs. Other possible explanations for the observations of Baker et al. (1991) and Sanders et al. (1996) include resuspension of CB-rich nepheloid materials and advective loss of dissolved material relative to CB-rich particulate material. Resuspension of material during top-to-bottom mixing is possible in these lakes, though it is unclear why this behavior does not affect the organic carbon and PCBs equally unless preferential remobilization of CB-rich particles is occurring. Even so, the possibility of microbial-mediated changes in organic matter composition leads to the hypothesis that organisms within the microbial loop can affect the cycling of PCBs.

Data in the previous chapter showed production of surface-active material during protozoan grazing. Surface-active material may have an enhanced ability to sorb PCBs over other material due to its amphiphilic (both hydrophobic and hydrophilic) structure (Grimberg et al., 1995; Mayer et al., 1996; Tiehm et al., 1997; Mayer et al., 1999).

Aggregates of this material may assume micelle-like structures with hydrophobic interiors. Because of this tendency, environmental remediation technologies have used surfactants to solubilize organic contaminants from soil particles (Tiehm et al., 1997). Surfactant concentrations used in these instances are in the $\mathrm{g} / \mathrm{L}$ range, depending on the specific surfactant. These concentrations greatly exceed those in this study (see Chapter 4 and Table 5-1).

\subsubsection{Goal}

The previous chapter showed that three protozoan grazers were capable of producing dissolved organic material that was different than that in bacterial and Vineyard Sound seawater controls. This study presented in this chapter ascertains the affinity of "grazer-enhanced" DOM for PCBs relative to bacterial and seawater DOM. 
The affinity of culture filtrates for PCBs was determined using closed-system vessels with a common headspace. The degree of CB-DOC association of different culture filtrates $(<0.2 \mu \mathrm{m})$ was determined using these experimental vessels and compared to bulk DOC concentrations and surfactant activities. Equilibrium partition coefficients were calculated for bulk DOC in each filtrate and compared as a function of prey growth substrate and time of collection.

\subsection{Methods}

\subsubsection{Aliquot collection}

The organisms studied in this chapter were the same as in Chapter 4 - the ciliate, Uronema sp. (clone: BBCil), the flagellate, Cafeteria sp. (clone: Cflag), and the flagellate, Paraphysomonas imperforata (clone: VS1). The prey for all cultures was Halomonas halodurans. The growth substrate for the prey was $0.04 \%$ yeast extract in $0.2 \mu \mathrm{m}$-filtered sterile Vineyard Sound seawater (VSW) unless otherwise specified. As

discussed in Chapter 4, yeast extract was used as the prey growth substrate because it is a complex mix of organic compounds and thus more closely resembles the DOM present in natural systems. All excess yeast extract was removed by the rinsing protocol described in Chapter 2.

Protozoan cultures were inoculated with bacterial prey concentrates obtained as described in Chapters 2 and 4. Filtrates were collected for CB-headspace analysis either at $24 \mathrm{~h}$ (exponential growth stage for Uronema) or at the end of the experiment (stationary growth $-\geq 48 \mathrm{~h}$ for Uronema and $\geq 72 \mathrm{~h}$ for both flagellates). Filtrates were collected primarily by syringe-filtration through $25 \mathrm{~mm} 0.2 \mu \mathrm{m}$ surfactant-free cellulose acetate filters (Nalgene, Fisher Scientific). In early studies, some filtrates were collected with vacuum filtration through $47 \mathrm{~mm} 0.2 \mu \mathrm{m}$ polycarbonate membrane filters (Nuclepore, Whatman, Fisher Scientific). These filtrates are indicated as such in the data figures.

All filtrates were inoculated with radio-labeled congener $\# 77$ or ${ }^{14} \mathrm{C}$-TCB $\left({ }^{14} \mathrm{C}\right.$ $3,3^{\prime}, 4,4^{\prime}$-tetrachlorinated biphenyl, specific activity $=52.1 \mu \mathrm{Ci} / \mu \mathrm{mol}$, courtesy of $\mathrm{J}$. Stegeman, WHOI, MA). The congener was added to the filtrate using an acetone carrier 
(about $150-200 \mu \mathrm{L}$ acetone per $220 \mathrm{~mL}$ filtrate). The concentration of congener in each filtrate ranged from 40 to $100 \mathrm{dpm} / \mathrm{mL}$ or 0.1 to $0.25 \mathrm{ng} / \mathrm{mL}$ (conversion factor $=2.52 \mathrm{X}$ $\left.10^{-3} \mathrm{ng} / \mathrm{dpm}\right)$.

For each culture studied during a size-fraction study, twice the volume needed was filtered through $0.2 \mu \mathrm{m}$ polycarbonate filters (as above). Half of this filtrate was then filtered through $0.02 \mu \mathrm{m}$ Anopore ${ }^{\circledR}$ filters (Whatman, Fisher Scientific). The $0.02 \mu \mathrm{m}$ filtrates were treated the same way as the $0.2 \mu \mathrm{m}$ filtrates for the remaining parts of the experiment.

Control solutions were $\mathrm{NaCl}$ solutions in Milli-Q water (40g in 2L Milli-Q water: Ionic strength $(\mathrm{I})=0.7 \mathrm{M})$. $\mathrm{NaCl}$ was combusted prior to use to remove any organic material in the solid salt. This solution was chosen such that the ionic strength of the solution would be the same as the seawater filtrates in the inner beaker.

\subsubsection{Headspace vessels}

Headspace vessels were manufactured according to a design modified from Brownawell (1997). Vessels were made of glass with ground-glass stoppers above the inner and outer beakers. Between experiments, vessels were cleaned by rinsing with Milli-Q water, methanol, acetone, and then Milli-Q water. Vessels were not washed with soap to avoid trace surfactant contamination. A schematic of the vessels is shown in Figure 5-1.

This design is basically a "beaker within a beaker". The experimental solution was spiked with a radioactive CB congener, equilibrated for $15 \mathrm{~min}$ to 1 hour and then transferred to the inner beaker. The outer beaker contained a control solution with no PCBs. As the vessel was mixed, the fraction of $\mathrm{CB}$ congener that is truly dissolved in the inner beaker diffused across the air-water interface into the overlying headspace. This mass-transfer was governed by $\mathrm{K}_{\mathrm{H}}$, the Henry's Law constant. The same constant then governed the transfer of PCBs into the control solution in the outer beaker. After approximately $36-40 \mathrm{~h}$, the two beakers were equilibrated with one another and the dissolved concentrations in each beaker were equivalent. The time frame of 
equilibration was ascertained by monitoring the transfer of PCBs from the inner to the outer beaker over time. On average, the ratios reached a steady value after approximately 36-40h (Figure 5-2). The transfer of PCBs in this system was governed by the following equation:

$$
[C B]_{\text {diss, immer }} \stackrel{K_{\| \prime}}{\longleftrightarrow}[C B]_{a i r} \stackrel{K_{\| \prime}}{\longleftrightarrow}[C B]_{\text {diss, outer }}
$$

One of the advantages of this method is that $\mathrm{K}_{H}$ and $[\mathrm{CB}]_{\text {air }}$ does not need to be known. The outer beaker serves as a concentration mechanism for the analysis of PCBs transferred into the headspace and so the analytical difficulties associated with determining $\mathrm{K}_{\mathrm{H}}$ and/or $[\mathrm{CB}]_{\text {air }}$ are circumvented.

\subsubsection{Time points and analysis}

At each time point, $10 \mathrm{~mL}$ samples were removed from each beaker compartment, combined with $7 \mathrm{~mL}$ scintillation cocktail (ScintiVerse II, Fisher Scientific) and counted on a Beckman 500 scintillation counter. At the end of each experiment, vessels wer rinsed with $10 \mathrm{~mL}$ Milli-Q water and $10 \mathrm{~mL}$ acetone to remove any wall-associated ${ }^{14} \mathrm{C}$ TCB. Wall rinses were combined and split into two vials, each containing $7 \mathrm{~mL}$ scintillation fluid and analyzed on the scintillation counter. Wall losses were generally $25-35 \%$ of the total initial activity. Total activities in the samples ranged from 200 to $1800 \mathrm{dpm}$. Activities in VSW blank samples ranged from $35 \mathrm{dpm}$ to $75 \mathrm{dpm}$ with an average of $55 \pm 7 \mathrm{dpm}$. Experimental samples were generally 2-3 X the blank. Blanks were subtracted from samples prior to further analysis. Six blanks were analyzed per experiment.

The ratio of the total activity in the inner and outer beakers was calculated and converted to a non-dimensional paramter, $C^{*}$. The equations used for this calculation follow.

$$
\begin{gathered}
\text { (1) } C^{*}=\frac{\text { Ratio }-1}{\text { Ratio }} * 100 \% \\
\text { (2) Ratio }=\frac{[C B]_{\text {Total,imer }}}{[C B]_{\text {Yotat, outer }}}=\frac{[C B]_{\text {diss, ,mmer }}+[C B]_{C D O M}}{[C B]_{\text {diss, outer }}}
\end{gathered}
$$




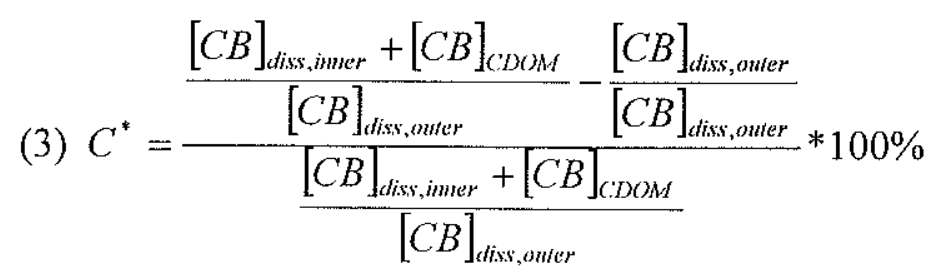

At equilibrium, the simplifying calculation of $[\mathrm{CB}]_{\text {diss,inner }}=[\mathrm{CB}]_{\text {diss,outer }}$ can be made. Thus $\mathrm{C}^{*}$ becomes the "percent associated with $\mathrm{C} / \mathrm{DOM}$ ".

$$
\text { (4) } C^{*}=\frac{[C B]_{C D O M}}{[C B]_{\text {diss, } \text {, hmer }}+[C B]_{C D O M}} * 100 \%=\% w C D O M
$$

The percent within the C/DOM class can be plotted versus time for specific headspace experiments (see Figure 5-2A-D). The assumption in the above calculation that $[C B]_{d i s s, i n n e r}=[C B]_{d i s s, o u t e r}$ was valid only at equilibrium. Clearly, this assumption was not appropriate during the early part of the headspace experiment, so $\mathrm{C}^{*}$ was plotted to give an indication of the time needed to achieve equilibrium in these vessels.

The parameter, $\mathrm{C}^{*}$ at $50 \mathrm{~h}$, was chosen to allow comparison of partitioning or percent within the C/DOM class in different experiments. In cases where samples were not taken at exactly $50 \mathrm{~h}$, the ratio was linearly interpolated from the ratios at the two time points on either side of $50 \mathrm{~h}$. The equation governing this manipulation is:

$$
\text { (5) } R_{t=50}=R_{u}+\left(50-t_{u}\right) * \frac{R_{b}-R_{u}}{t_{b}-t_{a}}
$$

where $\mathrm{R}_{\mathrm{a}}$ and $\mathrm{R}_{\mathrm{b}}$ are the ratios at the time prior to $50 \mathrm{~h}(a)$ and the time after $50 \mathrm{~h}(b)$, respectively, and $t_{a}$ and $t_{b}$ are the times of sample collection (hours).

As stated above, the outer beaker contained the control solution, $0.7 \mathrm{M} \mathrm{NaCl}$ in Milli-Q water. This solution was chosen such that the ionic strengths of the inner and outer solutions were equivalent. This choice of outer solution does not address the difference in salt ion composition in the two solutions. It is possible that the presence of sulfate and other large ions may have a greater "salting-out" effect than the smaller ions of sodium and chloride. This effect would serve to drive the PCBs out of the VSW into the $\mathrm{NaCl}$ solution regardless of the organic composition/concentration in the inner solution. Estimates of this "salting out" effect range up to a factor of two increase in $\mathrm{K}_{\text {org }}$ 
for nonpolar compounds (Schwarzenbach et al., 1993). This corresponds to about $0.3 \log$ units which is within the range observed in the average $\mathrm{K}_{\mathrm{p}}$ 's calculated in this system (see Results section 5.3.1.). However, this effect would be a constant offset (downward) for all the data since all experimental solutions were VSW.

The effect of the different salt composition in the control solution and VSW on the saturated activity coefficient, $\gamma_{\mathrm{w}, \text { salt }}$, , was calculated. First, the activity coefficient at saturation, $\gamma_{\mathrm{w}, \text { salt }}$ sat , can be calculated from the concentration at saturation (in the presence of salt), $\mathrm{C}_{\mathrm{w}, \text { salt }}$ sat , with the following equation from Schwarzenbach et al. (1993):

$$
\text { (6) } \gamma_{w, \text { salt }}^{\text {sut }}=\frac{1}{C_{w, \text { salt }}^{\text {sat }} * 0.018}
$$

where 0.018 is the molar volume of water. $\mathrm{C}_{\mathrm{w}, \text { salt }}{ }^{\text {sat }}$ was derived from the Setschenow relationship employing the saturation concentration in pure water (see Chapter 2):

$$
\text { (7) } \log \left(\frac{C_{w}^{\text {sat }}}{C_{w, \text { salt }}^{s a t}}\right)=K^{s}[\text { salt }]_{t}
$$

where $K^{s}$ is the Setschenow constant and [salt $]_{t}$ is the total molar salt concentration. The Setschenow constant, $\mathrm{K}^{\mathrm{s}}$, will vary with salt composition. Thus the $\mathrm{K}^{\mathrm{s}}$ values for IUPAC \#77 had to be derived for $\mathrm{NaCl}$ (control solution) and for VSW (experimental solution). The $\mathrm{NaCl}$ value was derived by linear interpolation from $\mathrm{K}^{s}$ values for benzene (0.19) and naphthalene (0.22) and their relative total surface areas $\left(\mathrm{TSA}(\right.$ benz $)=110 \AA^{2}$ and TSA(naph) $=156 \AA^{2}$ ). For IUPAC $\# 77$, TSA equals $251 \AA^{2}$ (Hawker and Connell, 1988) and the resultant $\mathrm{K}^{\mathrm{s}}$ equals 0.28 .

$$
\text { (8) } \frac{K^{x}(\text { naph })-K^{x}(\text { benz })}{T S A(\text { naph })-T S A(\text { benz })}=\frac{K^{s}(77)-K^{s}(\text { naph })}{T S A(77)-T S A(\text { naph })}
$$

The $\mathrm{K}^{\mathrm{s}}$ value for IUPAC\#77 in VSW (0.35) was estimated similarly using $\mathrm{K}^{\mathrm{s}}$ and TSA values for naphthalene $\left(K^{s}=0.25\right)$ and pyrene $\left(K^{S}=0.31\right.$; $\left.T S A=213 \AA^{2}\right) . C_{w}{ }^{\text {sat }}$ was calculated from $\log \mathrm{K}_{\mathrm{ow}}$ (Hawker and Connell, 1988) according to the relationship:

(9) $\log C_{w}^{s a t}=\frac{\log K_{o w}-0.78}{-0.85}$ 
$\mathrm{C}_{\mathrm{w}}{ }^{\text {sat }}$ for IUPAC \#77 was calculated to be $2.72 \times 10^{-7} \mathrm{~mol} / \mathrm{L}$. Using all the above values, $\gamma_{\mathrm{w} \text {,salt }}^{\text {sat }}$ was calculated to be $3.21 \times 10^{8} \mathrm{~L} / \mathrm{mol}$ for the $\mathrm{NaCl}$ solution and $3.18 \times 10^{8} \mathrm{~L} / \mathrm{mol}$ for seawater. The difference in these two activity coefficients is approximately $1 \%$, suggesting that the activities of ${ }^{14} \mathrm{C}-\mathrm{TCB}$ will be approximately the same in the two solutions and no correction for different salt composition is necessary.

\subsubsection{Brief overview of parameter analyses}

Binding potentials in all culture filtrates were compared with DOC concentrations and surfactant activities. These parameters were measured using the analyses described in detail in Chapter 4. DOC concentrations were measured using high-temperature combustion (Peltzer and Brewer, 1993) at UMass-Boston (R. Chen lab). Surfactant activities were measured using an electrochemical method that expresses surfactant activity in terms of Triton X-100 equivalents (Hunter and Liss, 1981).

\subsection{Results}

\subsubsection{Partition coefficients ( $\left.K_{D O C}\right)$ in culture filtrates}

Partition coefficients $\left(K_{\mathrm{DOC}}\right)$ were compared among culture filtrates to see if "grazer-enhanced" DOM had a higher affinity for PCBs than either bacterial DOM or DOM found in VSW. These coefficients were calculated for each filtrate using the bulk DOC concentration and the percent associated with C/DOM according to the following equation:

$$
K_{H x:}=\frac{f_{B}}{[D O C]^{*}\left(1-f_{B}\right)}
$$

where: $K_{D O C}$ is the partition coefficient ((mass CB/g OC) $/($ mass $\left.C B / m L)\right), f_{B}$ is the fraction associated with C/DOM, and [DOC] is the DOC concentration in $\mathrm{g} / \mathrm{mL}$ (equals $10^{6} \mathrm{mg} / \mathrm{L}$ ). Partition coefficients for all culture filtrates are shown in Table 5-1. Wall loss fractions were not included with the C/DOM fraction because (1) they were relatively constant and (2) the wall loss is a combination of adsorption of dissolved PCBs to the glass surface and association with DOM adsorbed to the glass. The average log $\mathrm{K}_{\mathrm{DOC}}$ 's 


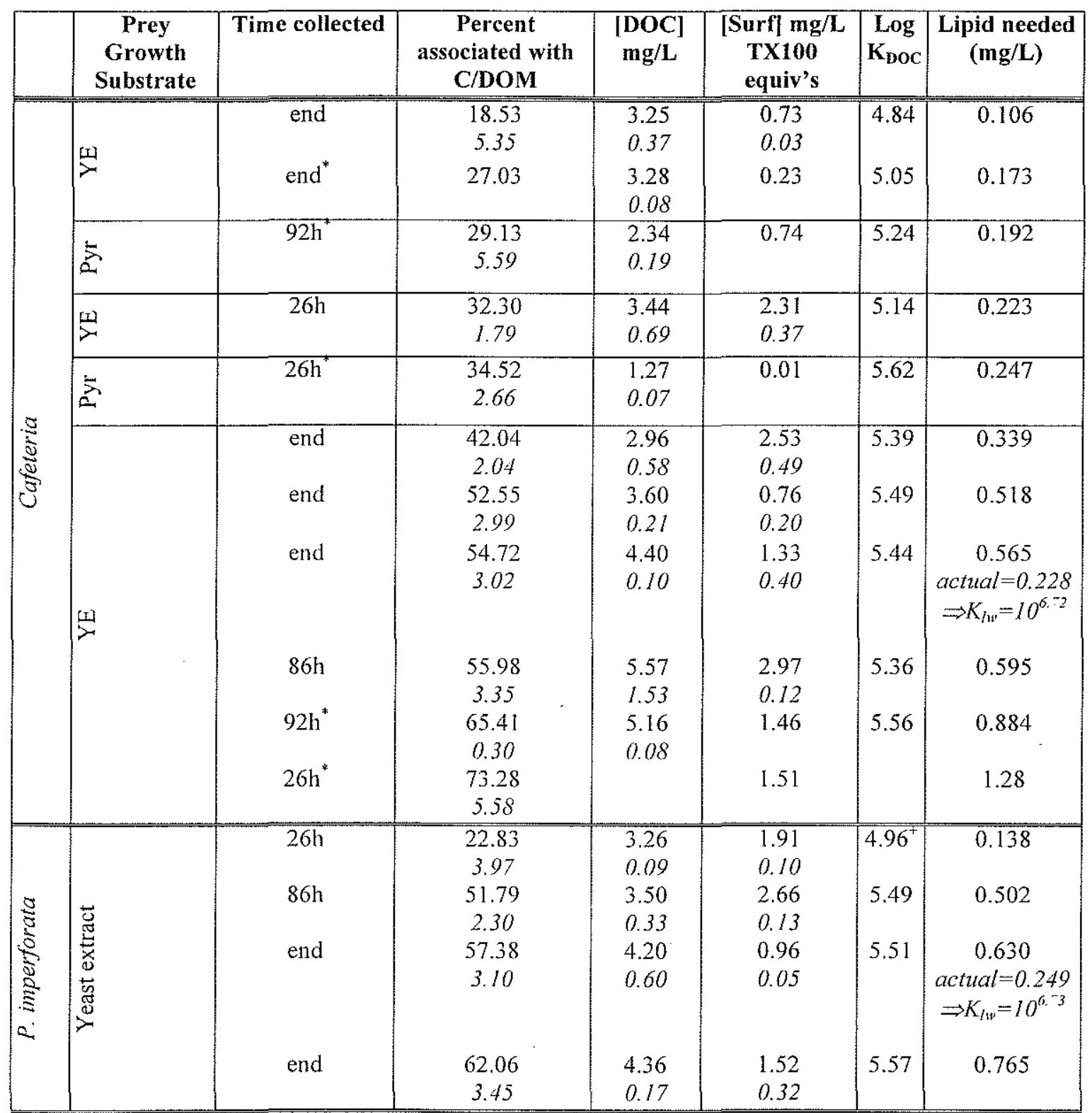

Table 5-1. Data from all headspace experiments.

The binding data from all headspace experiments is presented. The columns denote the (1) organism from which the culture filtrate is derived (H.halodurans is the bacterial control), (2) the growth substrate of the bacterial prey, (3) the percent associated with C/DOM, (4) the DOC concentration (mg/L), (5) the surfactant activity (mg/L TX100 equivalents), (6) the calculated $\log K_{P}$ for the bulk DOC, and (7) the amount lipid needed to generate the fraction associated with C/DOM $(\mathrm{mg} / \mathrm{L})$. The numbers in italics are the standard deviations $(\sigma)$ from the mean $(n=2$ or 3$)$. The data for each organism is arranged in order of increasing percent associated with $\mathrm{C} / \mathrm{DOM}$.

* - Filtrate collected using vacuum filtration through $0.2 \mu \mathrm{m}$ Nuclepore filter instead of syringe filtration through $0.2 \mu \mathrm{m}$ surfactant-free cellulose acetate.

+ - Outlier: discarded because data point falls $\geq 3 \sigma$ from mean of remaining data. 
were $5.31 \pm 0.24(n=10)$ for Cafeteria samples, $5.52 \pm 0.05(n=3)$ for $P$. imperforata samples (without outlier), $5.38 \pm 0.23(n=10)$ for Uronema samples, $5.08 \pm 0.08(n=4)$ for H. halodurans samples (without outlier) and $4.60 \pm 0.16(n=2)$ for VSW controls. These partition coefficients were used to predict percent associated with C/DOM and the predictions were compared to this data set (Figure 5-3). Outliers were tested by calculating the mean without the suspected outlier. If the suspected data point was more than $3 \sigma$ away from the mean, it was discarded.

\begin{tabular}{|c|c|c|c|c|c|c|c|}
\hline & $\begin{array}{c}\text { Prey } \\
\text { Growth } \\
\text { Substrate } \\
\end{array}$ & Time collected & $\begin{array}{c}\text { Percent } \\
\text { associated with } \\
\text { C/DOM } \\
\end{array}$ & $\begin{array}{c}\mathrm{DOC}] \\
\mathrm{mg} / \mathrm{L}\end{array}$ & $\begin{array}{c}\text { [Surf] mg/L } \\
\text { TX100 } \\
\text { equiv's }\end{array}$ & $\begin{array}{l}\text { Log } \\
\text { K }_{\text {DOC }}\end{array}$ & $\begin{array}{l}\text { Lipid needed } \\
\qquad(\mathrm{mg} / \mathrm{L})\end{array}$ \\
\hline & 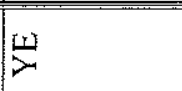 & end $^{*}$ & 33.19 & $\begin{array}{l}3.43 \\
0.49\end{array}$ & $\begin{array}{l}0.47 \\
0.08\end{array}$ & 5.16 & 0.232 \\
\hline & 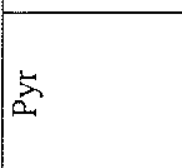 & $\begin{array}{l}26 \mathrm{~h}^{*} \\
92 \mathrm{~h}^{*}\end{array}$ & $\begin{array}{c}37.87 \\
8.07 \\
38.51 \\
6.11\end{array}$ & $\begin{array}{l}2.80 \\
0.10 \\
1.92 \\
0.42\end{array}$ & 0.06 & $\begin{array}{l}5.34 \\
5.51\end{array}$ & $\begin{array}{l}0.285 \\
0.293\end{array}$ \\
\hline & \multirow{12}{*}{ 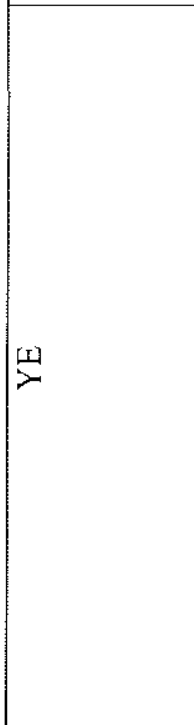 } & end & $\begin{array}{l}38.95 \\
4.28\end{array}$ & & $\begin{array}{l}2.60 \\
0.08\end{array}$ & & 0.298 \\
\hline & & $92 \mathrm{~h}^{*}$ & $\begin{array}{l}47.15 \\
0.95\end{array}$ & $\begin{array}{l}4.03 \\
0.14\end{array}$ & 0.75 & 5.35 & 0.417 \\
\hline & & end ${ }^{*}$ & $\begin{array}{r}48.19 \\
2.64\end{array}$ & $\begin{array}{l}9.36 \\
0.16\end{array}$ & 1.19 & 5.00 & 0.435 \\
\hline \multirow{2}{*}{$\begin{array}{l}\text { D } \\
\text { 5 } \\
5 \\
0 \\
5\end{array}$} & & $26 \mathrm{~h}$ & 49.66 & 3.94 & 6.18 & 5.40 & 0.461 \\
\hline & & & 7.24 & 0.25 & 0.10 & & \\
\hline & & $86 \mathrm{~h}$ & $\begin{array}{c}57.72 \\
3.16\end{array}$ & $\begin{array}{c}10.26 \\
1.05\end{array}$ & $\begin{array}{l}4.90 \\
0.35\end{array}$ & 5.12 & 0.638 \\
\hline & & end & 62.47 & 3.61 & 0.59 & 5.66 & 0.778 \\
\hline & & & 7.49 & 0.20 & 0.03 & & \\
\hline & & end & $\begin{array}{c}64.30 \\
0.22\end{array}$ & & $\begin{array}{l}7.76 \\
0.13\end{array}$ & & 0.842 \\
\hline & & end & 64.88 & 5.03 & 2.03 & 5.57 & 0.864 \\
\hline & & & 7.72 & 0.11 & 0.30 & & \\
\hline & & $26 \mathrm{~h}^{*}$ & $\begin{array}{c}67.09 \\
8.19\end{array}$ & $\begin{array}{l}4.25 \\
0.16\end{array}$ & 1.47 & 5.68 & 0.953 \\
\hline \multirow{8}{*}{ 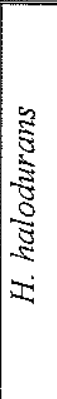 } & \multirow{4}{*}{ 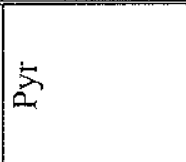 } & $95 \mathrm{~h}$ & 23.07 & 2.21 & 0.12 & 5.13 & 0.140 \\
\hline & & & 12.69 & 0.57 & & & \\
\hline & & $26 \mathrm{~h}$ & 23.43 & 2.16 & 0.11 & 5.15 & 0.143 \\
\hline & & & 4.78 & 0.99 & & & \\
\hline & \multirow{4}{*}{ 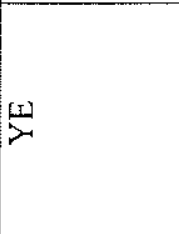 } & $24 \mathrm{~h}$ & $\begin{array}{c}28.07 \\
5.89\end{array}$ & $\begin{array}{l}4.11 \\
1.72\end{array}$ & 0.28 & 4.98 & 0.182 \\
\hline & & $26 \mathrm{~h}$ & 30.48 & 3.69 & 0.75 & 5.07 & 0.205 \\
\hline & & & 14.94 & 0.66 & & & \\
\hline & & $41 \mathrm{~h}$ & $\begin{array}{l}41.48 \\
10.90\end{array}$ & $\begin{array}{l}3.40 \\
0.41\end{array}$ & 1.12 & $5.32^{+}$ & 0.331 \\
\hline
\end{tabular}

Table 5-2. Data from all headspace experiments - Uronema and H. halodurans.

Data prepared in same manner as Table 5-1. 


\subsubsection{Comparison of binding potential with bulk DOC and surfactants}

Data from all experiments are compared to bulk DOC concentrations in Figure 55. With the exception of two Uronema points, the data on this plot occupied a rather tight range. The VSW controls showed the smallest percent associated with C/DOM. The bacterial controls were generally lower than the protozoan grazing culture filtrates. A few of the Cafeteria culture filtrates had similar CB affinities as the bacterial controls. The $P$. imperforata point occurring in this range was discarded in the previous section because it was an outlier relative to the other three cultures tested. The relationship between DOC concentration and $\mathrm{C}^{*}$ was not linear as can be seen by the lines drawn in Figure 5-3. However, it is clear from this figure that increased CB-DOC associations were occurring that cannot be explained simply by increases in DOC concentration. This implied that a compositional or structural change had occurred in the culture DOC that increased its affinity for PCBs. Similar behavior was observed in the surfactant data (Figure 5-4). There was a great deal more scatter in the data set.

The surfactant-water partition coefficient for IUPAC \#77 was not calculated because the surfactant activity is not based on mass of a particular component of DOC. Instead, surfactant activity is an operation definition describing a property of the material in question. A partition coefficient relating this property to binding affinity on a strictly quantitative basis would have little chemical significance. Lipid-water partition coefficients were calculated for the two culture filtrates for which I measured lipid concentrations (Tables 5-1 and 5-2). Both values calculated $\left(10^{6.72}\right.$ and $10^{6.73}$ ) were higher than $\mathrm{K}_{\text {ow }}\left(10^{6.36}\right)$ and were most likely inflated by contributions from non-lipid material.

\subsubsection{Calculation of potential lipid contribution}

Lipid material has a much higher partition coefficient $\left(\mathrm{K}_{\mathrm{Iw}}\right)$ than bulk DOC (Swackhamer and Skoglund, 1993) so less material would be needed to generate the same fraction bound as bulk DOC. For this study, I have calculated the amount of lipid 
material that would be needed to give the observed fraction bound in each filtrate (Tables 5-1 and 5-2). The equation used for this calculation is:

$$
\text { (11) } \quad[\text { Lip }]=\frac{f_{B}}{K_{/ w} *\left(1-f_{B}\right)}
$$

where: [Lip] is the lipid concentration in $\mathrm{g} / \mathrm{mL}$ (equals $10^{6} \mathrm{mg} / \mathrm{L}$ ) and $\mathrm{K}_{\mathrm{lw}}$ is the lipidwater partition coefficient ( $10^{6.33}$ from equation in Swackhamer et al. (1993)). This equation does not include contributions from $\mathrm{CB}$ associations with DOC with lower affinity (e.g., $\log K_{o c}=4.6$ ). This consideration would generate lower lipid numbers than in Tables 5-1 and 5-2.

In general, culture filtrates with low "percent associated with C/DOM" ( $<35$ $40 \%$ ) required less or similar lipid concentrations than were measured to explain the observed binding. Filtrates with $>35-40 \%$ "association with C/DOM" required more lipid than was measured in similar culture filtrates. As presented in Chapter 4, culture filtrates from cultures of Uronema, Cafeteria, and P. imperforata contained approximately $0.2 \mathrm{mg} / \mathrm{L}$ lipid material (defined by extraction with $\mathrm{CHCl}_{3}: \mathrm{MeOH}$ ). Therefore, it must be presumed that non-lipid material in the culture filtrates was enhancing the binding affinity of the bulk material. The nature of this remaining material cannot be ascertained with the analytical methods used in this study. The lipid extraction procedure accounted for $10-20 \%$ of bulk DOC so the remaining $80-90 \%$ is contributing to the increased affinity, either by large quantities of low-affinity material or small quantities of high-affinity material. In short, the binding observed in the majority of the culture filtrates tested could not be explained simply by the presence of lipid material. Binding affinities of C/DOM in cultures inoculated with pyruvate-grown prey were lower than some of those fed YE-grown prey. However, low binding potentials were observed with YE-grown prey as well. No significant correlations can be made between binding affinity and prey growth substrate because a systematic survey of growth substrates was not performed. 


\subsubsection{Size-fraction study}

The size-dependence of the binding material was addressed by comparing binding potentials between $0.2 \mu \mathrm{m}$ and $0.02 \mu \mathrm{m}$ culture filtrates (Figure 5-6). In all cultures studied, the binding potential was significantly lower in the $0.02 \mu \mathrm{m}$ filtrates than in the $0.2 \mu \mathrm{m}$ filtrates. The Anopore filters are aluminum oxide and so should be negatively charged at seawater $\mathrm{pH}(\mathrm{pH} 8)$. Surfactants should also be negatively charged at $\mathrm{pH} 8$ and therefore should not be selectively removed by charge interactions with the filter. The effect of the size-fractionation on DOC concentrations and surfactant activities could not be ascertained because of filter bleeds (see Chapter 4). However, it is interesting to note the decrease in binding potential with reduction in size.

\subsection{Discussion}

\subsubsection{Comparison of grazing filtrates to bacterial and VSW controls}

Previous studies have measured the partition coefficient for seawater bulk DOC, $\mathrm{K}_{\mathrm{oc}}$ (Table 5-2). My values compared well with these estimates. This similarity to previous work showed that the background DOC from VSW was not contributing

\begin{tabular}{|c|c|}
\hline $\begin{array}{c}\text { Log } \mathrm{K}_{\text {Doc }} \text { predicted } / \\
\text { observed }\end{array}$ & Reference \\
\hline $0.8 * \log K_{o c}=4.3$ & Gustafsson (1997) \\
\hline $4.6 \pm 0.16$ & This study (VSW) \\
\hline 4.7 & Brownawell (1986) \\
\hline $\begin{array}{l}0.95^{*} \log \mathrm{K}_{\mathrm{oc}}=5.09 \\
\text { (anoxic porewater) }\end{array}$ & $\begin{array}{l}\text { Adapted from Hunchak- } \\
\text { Kariouk et al. (1997) }\end{array}$ \\
\hline $5.08 \pm 0.08$ & $\begin{array}{c}\text { This study } \\
\text { (H.halodurans })\end{array}$ \\
\hline $5.31 \pm 0.24$ & $\begin{array}{l}\text { This study } \\
\text { (Cafeteria) }\end{array}$ \\
\hline $5.38 \pm 0.23$ & $\begin{array}{l}\text { This study } \\
\text { (Uronema) }\end{array}$ \\
\hline $5.52 \pm 0.05$ & $\begin{array}{l}\text { This study } \\
\text { (P.imperforata) }\end{array}$ \\
\hline $\begin{array}{c}1.13^{*} \log \mathrm{K}_{\mathrm{cc}}=6.05 \\
\text { (oxic porewater) }\end{array}$ & $\begin{array}{l}\text { Adapted from Hunchak- } \\
\text { Kariouk et al. (1997) }\end{array}$ \\
\hline
\end{tabular}

Table 5-3. Comparison of this study's values for $K_{D O C}$ of seawater DOC with literature values. 
significantly to the high binding potentials observed in the culture filtrates. Partition coefficients calculated for bulk DOC in grazing culture filtrates were higher than those calculated for bacterial and VSW controls (Tables 5-1 and 5-2). This suggested that freshly-produced "grazer-enhanced" C/DOM was a better sorbent than either bacterial exudates or "aged" VSW DOC.

A number of hypotheses can be proposed to explain this observation. First, there has been an increase in lipid material that is forming aggregates with resultant microenvironments conducive to $\mathrm{CB}$ dissolution. However, data from Chapter 4 did not indicate an increase in lipid material over the bacterial control. In addition, one can see from Table 5-1 that most of the culture filtrates studied needed more lipid than was measured to generate the observed fractions sorbed. Table 5-3 is a comparison of lipid concentrations in seawater with this study and other culture studies. Dissolved lipid concentrations in field samples are comparable to those measured in this study (see Chapter 4 for additional data). The parity of the lipid concentrations suggests that the enhanced binding observed in the protozoan culture filtrates is not a result of increased lipid concentrations alone. Clearly, material with better sorbing capacities is produced in "grazer-enhanced" DOM. We know from other work (Gustafsson and Gschwend, 1997) that material does not need to be "lipid" (this class is operationally defined - see Roose and Smedes (1996) for good discussion of this topic) in order to act as good sorbent for PCBs. It only needs to achieve a conformation that allows the formation of a microenvironment for dissolution (partial or complete) of PCBs. A number of biological macromolecules can achieve such a structure in natural environments (e.g., proteins, glycolipids, etc.). This material is present in bacterial cells and would be likely excretion products for protists and could explain the enhanced binding observed in these cultures. Lipid concentrations are in the dissolved phase, defined as $<1 \mu \mathrm{m}$, unless otherwise noted. "Lipids" are defined in each reference by extraction with an organic solvent. In most cases, the solvent system used is a variation on the method by Bligh and Dyer (1959). Other solvent protocols include extraction with dichloromethane (Lombardi and Wangersky, 1991). 


\begin{tabular}{|c|c|c|}
\hline $\begin{array}{l}\text { Dissolved lipid concentration } \\
\qquad(\mu \mathrm{g} / \mathrm{L})\end{array}$ & Site and season & Reference \\
\hline 18 & North Sea (Mar-June) & Kattner et al. (1983) \\
\hline $49.4-88.3$ & Northern Adriatic Sea (June) & Derieux et al. (1998) \\
\hline $49-190$ & $\begin{array}{l}\text { Scotian shelf } \\
\text { Atlantic west coast (Apr \& June) }\end{array}$ & Parrish et al. (1988) \\
\hline $60-160$ & Gulf of Mexico (Nov \& Feb) & Kennicutt \& Jeffrey (1981) \\
\hline 70 & Baltic Sea (winter) & Andersson et al. (1993) \\
\hline $73-299$ & Rhone River estuary (May) & Leveau et al. (1990) \\
\hline $100-200$ & North Adriatic Sea (Nov) & Gasparovic \& Cosovic (1994) \\
\hline $150-300$ & $\begin{array}{l}\text { Bedford Basin, } \\
\text { Atlantic west coast (Mar-Apr) }\end{array}$ & Parrish et al. (1988) \\
\hline 880 & Baltic Sea (spring bloom) & Andersson et al. (1993) \\
\hline $190-330(<0.2 \mu \mathrm{m})$ & $\begin{array}{c}\text { Cultures } \\
\text { (H.halodurans, Cafeteria, } \\
\text { P.imperforata, Uronema) }\end{array}$ & This study (Chapter 4) \\
\hline $3000(<0.7 \mu \mathrm{m})$ & $\begin{array}{l}\text { Diatom Culture } \\
\text { Chaetoceros gracilis }\end{array}$ & Lombardi \& Wangersky (1991) \\
\hline
\end{tabular}

Table 5-4. Lipid concentrations in seawater from different regimes compared to those in our study.

One such type of biological DOC might be surface-active material as observed in Chapter 4. The relationship between binding affinity of C/DOM in culture filtrates and their surfactant activities was not straightforward. There was a great deal of scatter around the linear regression, suggesting that surface-active material was not the only factor affecting binding affinity in these filtrates. The "plateau" effect shown by the three Uronema data points at high surfactant activities could be interpreted as saturation behavior. However, the operationally-defined measure of surfactant activity may not be directly related to CB binding affinity in DOM. CB binding affinity will be a function of the microenvironment formed within the conformation of the organic matter, whereas surfactant activity is an index for the ability of a material to adsorb onto the surface of a $\mathrm{Hg}^{0}$ drop. As mentioned above, meaningful surfactant-water partition coefficients for 
IUPAC \#77 could not be calculated. Nonetheless, the general trend of increased binding with increased surface activity is a significant finding, especially at the concentrations of surface active material measured in this study ( $\mathrm{mg} / \mathrm{L}$ as opposed to $\mathrm{g} / \mathrm{L}$ employed in other studies (Tiehm et al., 1997)).

\subsubsection{Implication for $P C B$ s in natural systems}

The results from this investigation are applicable to microbial loop-dominated systems such as the sediment-water interface of select coastal environments as well as

oligotrophic marine systems. Because commercial manufacture of PCBs has ceased, sediments currently pose the largest source of PCBs to the environment in many areas (NRC, 1979). During diagenesis, organic matter in the surficial sediments will release the associated PCBs. Pore-water flushing will bring these PCBs into the flocculant layer immediately above the sediment-water interface. This layer is incredibly rich in organic matter that has recently settled from the surface (Baker and Eisenreich, 1989) and is susceptible to resuspension events in shallow areas. The microbial loop is very active in the remineralization of organic matter in this layer.

The data in this study indicated that the material produced by protozoan grazers during grazing of bacteria can sorb PCBs more efficiently than the background DOC in seawater. At high enough concentrations, this material will then "trap" PCBs in suspended phase and increase the residence time of PCBs in the water column. Production of high CB-affinity material at the sediment-water interface by protozoan grazers may explain the enhanced remobilization of PCBs observed in the field studies of Baker et al. (1991) and Sanders et al. (1996). Only a fraction of the PCBs in the system will be buried because they are constantly being shuttled between labile organic pools. In addition to remineralization, some of the organic matter is buried. Since the PCBs are free to equilibrate among the organic pools present, they will be constantly moving to the labile, lipid-rich material and will not be buried at the same rate as bulk organic matter. As noted above, the binding affinity of "grazer-enhanced" DOM is species-independent. As such, the effect on $\mathrm{CB}$ speciation observed in this study does not rely on the presence 
of a single protist species. Instead, the results are applicable to protist assemblages, as found in natural systems. Certainly the production rates of different types of material will vary among protists (see Chapter 4). As long as the different species are all excreting somewhat similar material in terms of CB affinity, PCBs should be kept in the water column longer than expected from simple organic matter cycling.

An estimation of the effect of protozoan-derived DOM on the cycling of PCBs is premature at this stage. First, the exact nature and composition of the material most responsible for the enhanced binding needs to be ascertained. Second, the production rate needs to be measured during protozoan grazing experiments and understood as a function of protozoan growth stage, prey growth substrate and most importantly, protozoan species. Finally, the sinks for "grazer-enhanced" DOM should be identified and the resultant residence time of this high-affinity material should be calculated. With the above information, one could calculate the net production of "grazer-enhanced" DOM. Coupling the production estimate with the binding affinity data from this chapter, one could calculate the degree to which PCBs are "sequestered" in the suspended organic pool. Then, depending on the hydrodynamic properties of the system in question (e.g., advective transport fluxes), one could estimate the increased residence time of PCBs within the system as a direct result of protozoan-derived DOM. In the end, the increased residence time and net fluxes out of the sediment-water interface would need to be compared to other remobilization processes such as pore-water flushing, degradation of contaminant-rich particles during digestion by macrofauna and bioturbation.

\subsection{Conclusions}

From this work, I conclude that "grazer-enhanced" C/DOM was a better sorbent for CBs than bacterially-derived C/DOM or VSW background material. The affinity of this material for PCBs was species-independent and potentially growth substrateindependent. Measured lipid concentrations for similar culture filtrates were lower than predicted lipid concentrations from the observed binding affinity of "grazer-enhanced" DOM, suggesting that conformational as well as compositional changes may have 
occurred in the DOM in these cultures. Production of high CB-affinity material by protozoan grazers may explain the enhanced remobilization of PCBs observed in the field studies of Baker et al. (1991) and Sanders et al. (1996). 


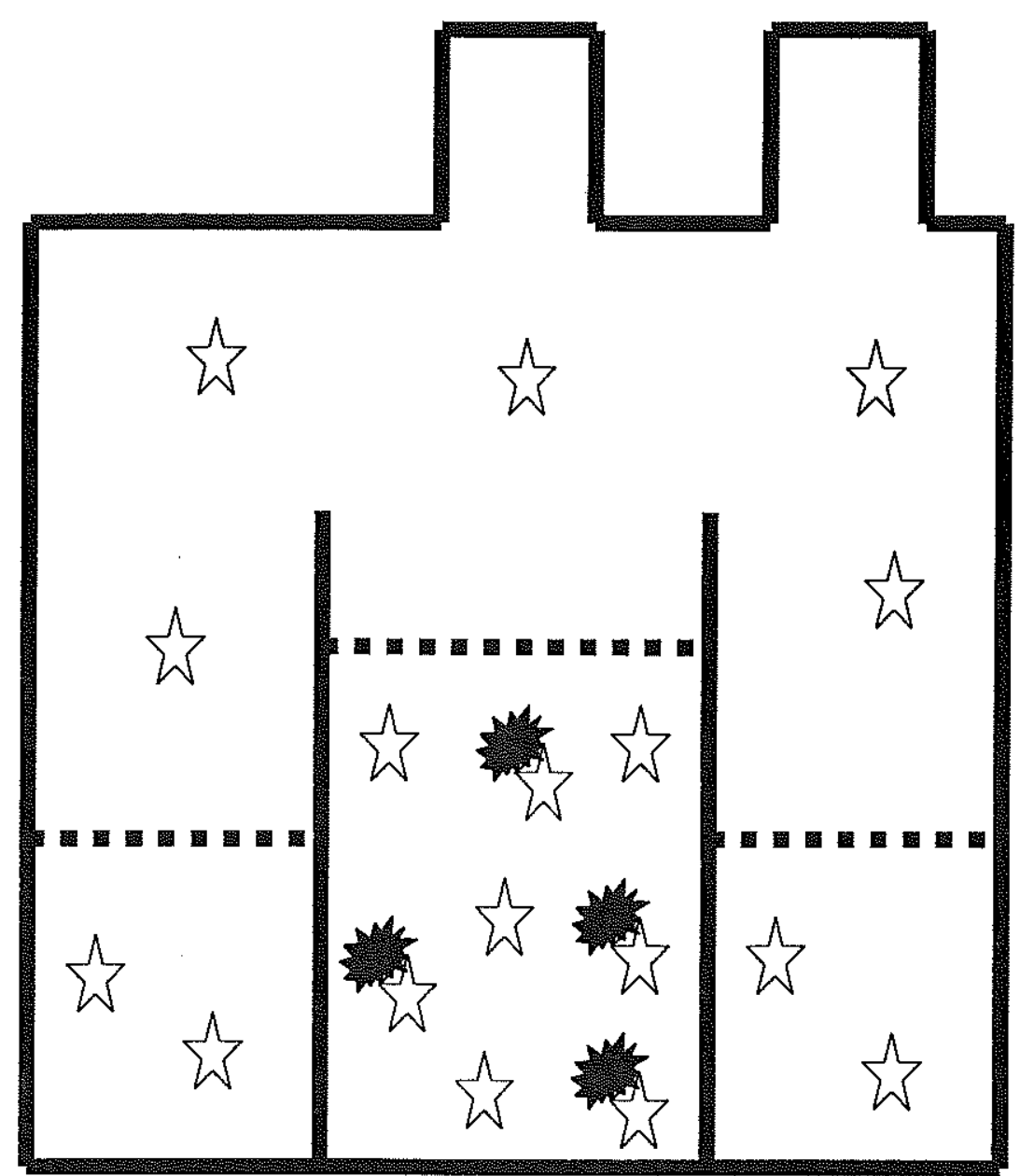

Figure 5-1. Schematic of headspace vessel.

This is basically a beaker within a beaker, modified from Brownawell (unpublished). The system is closed by glass stoppers at the top of the vessel. Aliquots of an experimental solution are in the inner beaker and a control solution is in the outer beaker. The PCBs (denoted by stars) can exist as free or bound (DOM denoted by black shapes) in the inner beaker. Only free PCBs are transferred into the headspace and then into the control solution in the outer beaker. Equilibrium between the inner and outer beakers is enhanced by mixing on a rotary table shaker which speeds transport across the air-water interface. 
A Cafeteria and Uronema on Pyruvate Hhalodurans - 24h grazing

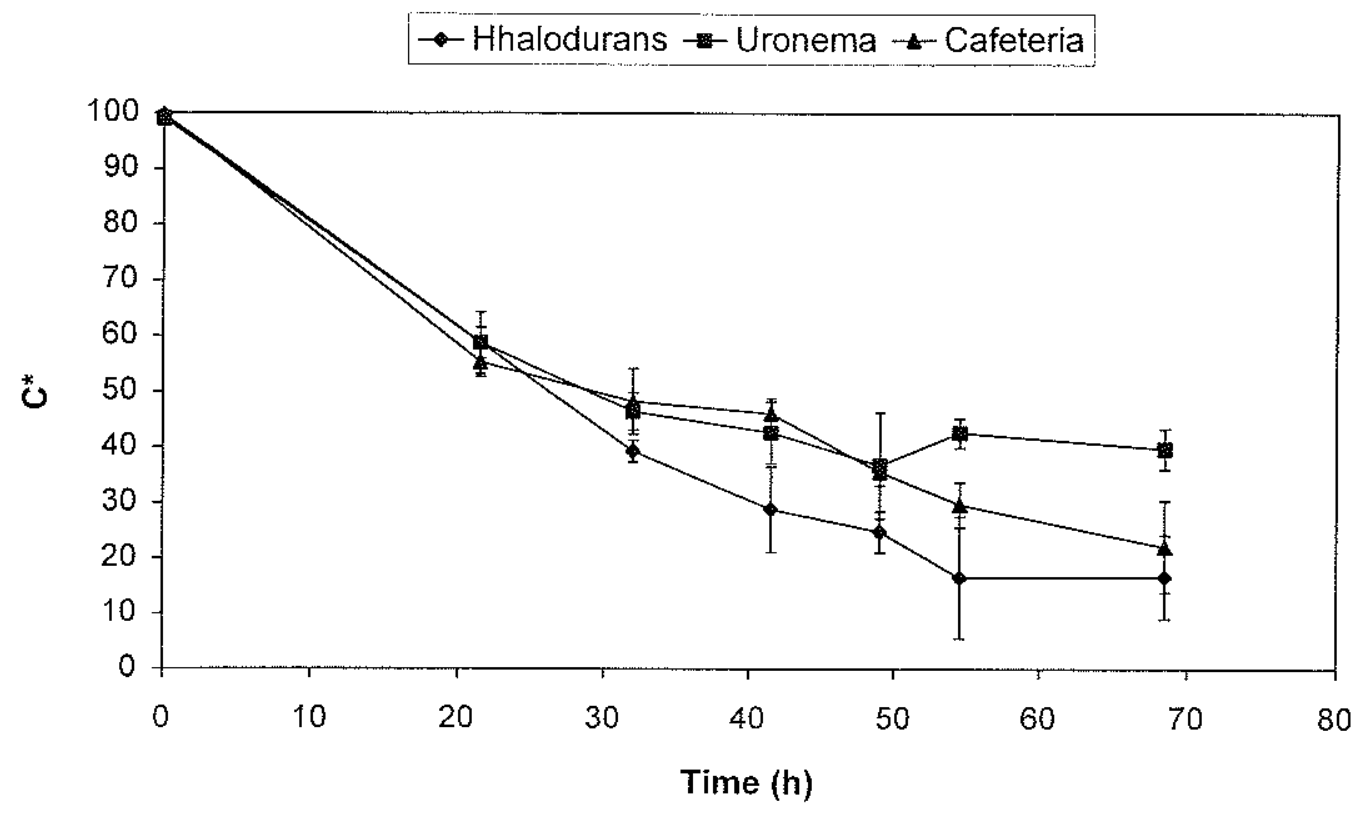

B Cafeteria and Uronema on Pyruvate Hhalodurans - 95h grazing

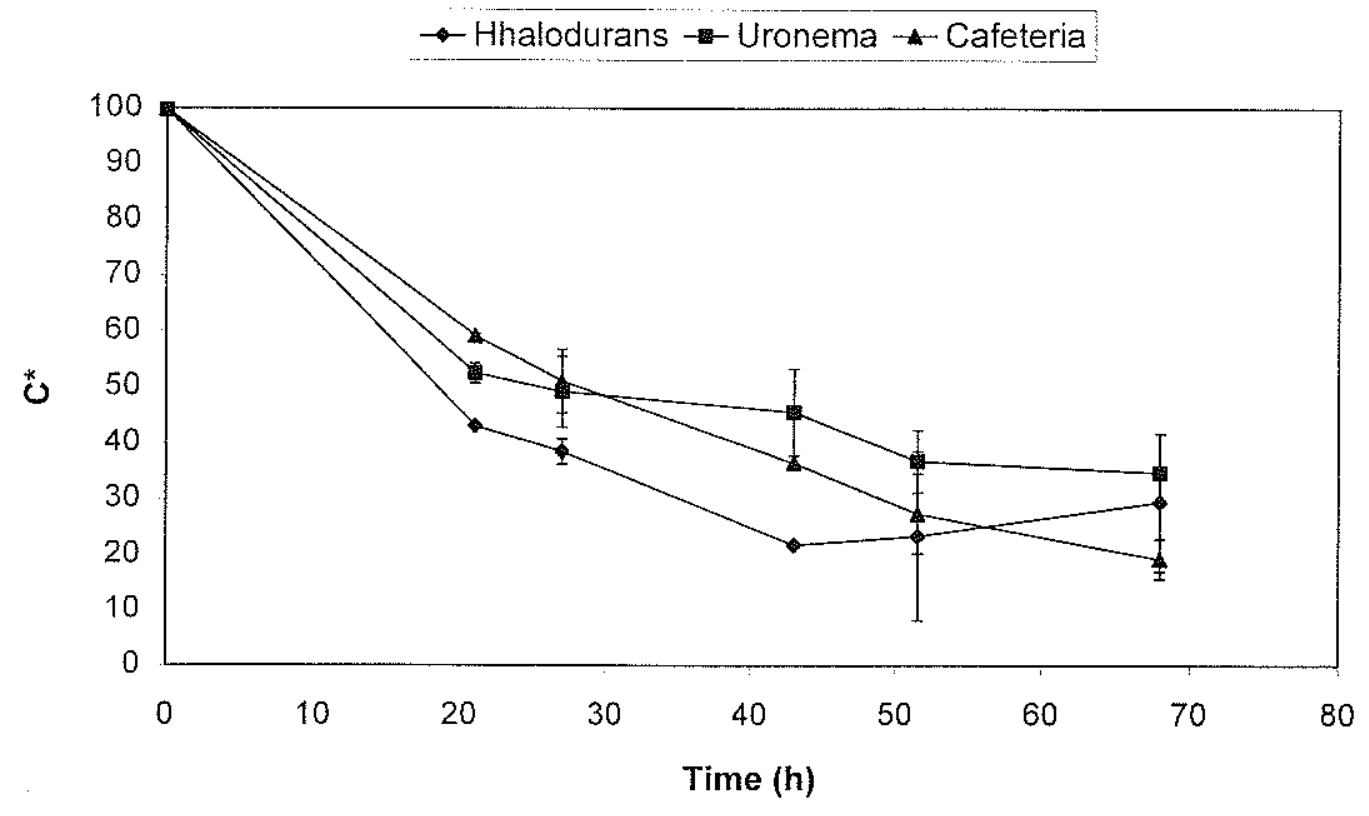



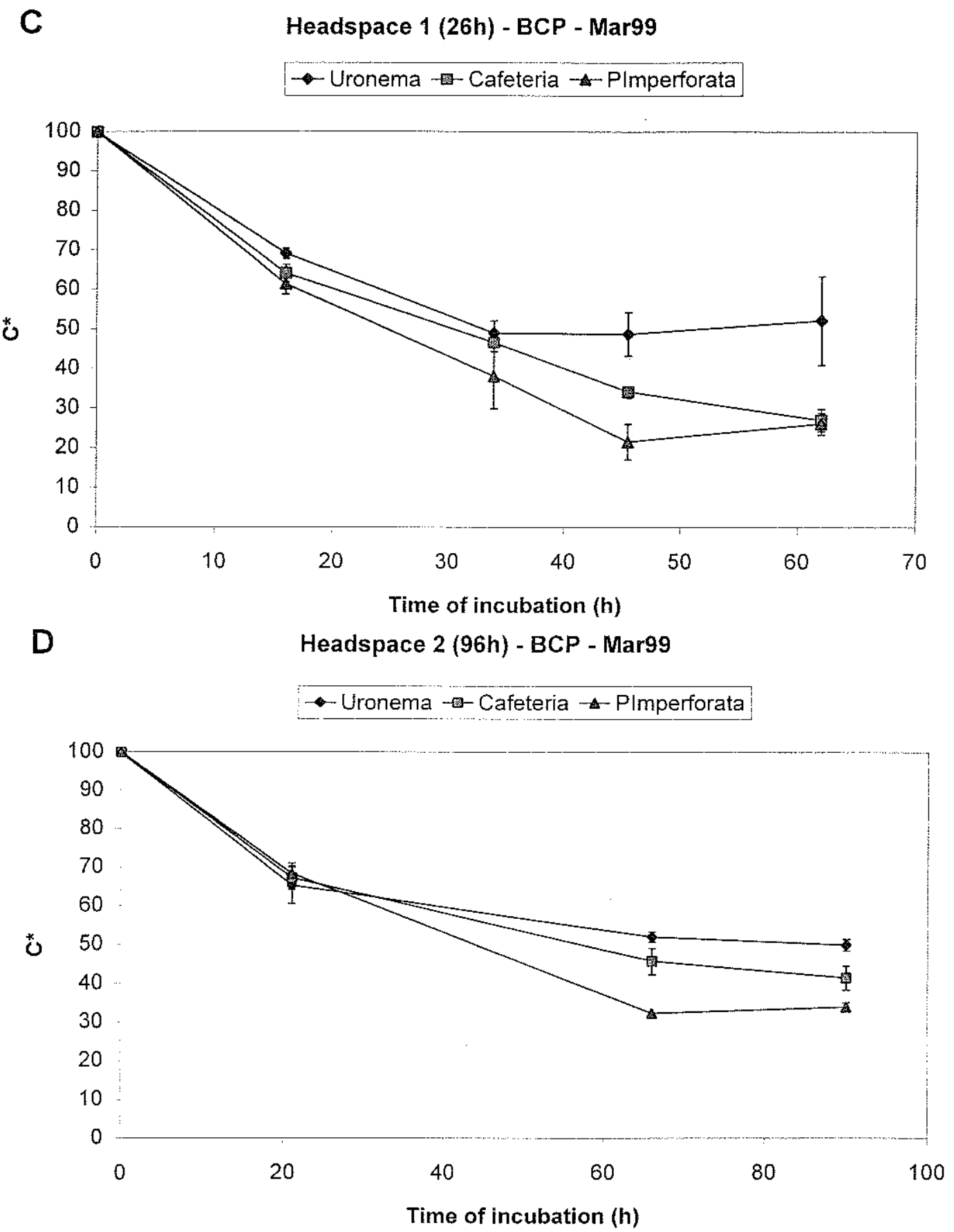

Figure 5-2. Representative figures of different headspace experiments.

These four graphs are representative figures of different headspace experiments. $\mathbf{A}$ and $\mathbf{B}$ are from a grazing experiment where Uronema (triangles) and Cafeteria (squares) were fed pyruvate-grown bacteria (Hhalodurans - diamonds). The filtrates for A were collected at the $24 \mathrm{~h}$ time point of the grazing culture and $\mathbf{B}$ was collected at the end of the experiment. $\mathbf{C}$ and $\mathbf{D}$ are from the interspecies comparison experiment discussed in Chapter $4(\mathrm{C}=24 \mathrm{~h}$ and $\mathrm{D}=$ end). Filtrates from all protozoan cultures are shown: Uronema (diamonds), Cafeteria (squares), and $P$. imperforata (triangles). 


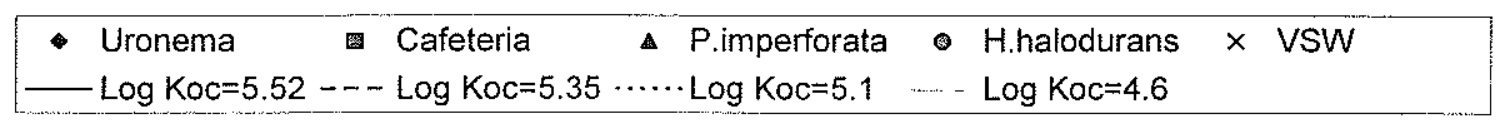

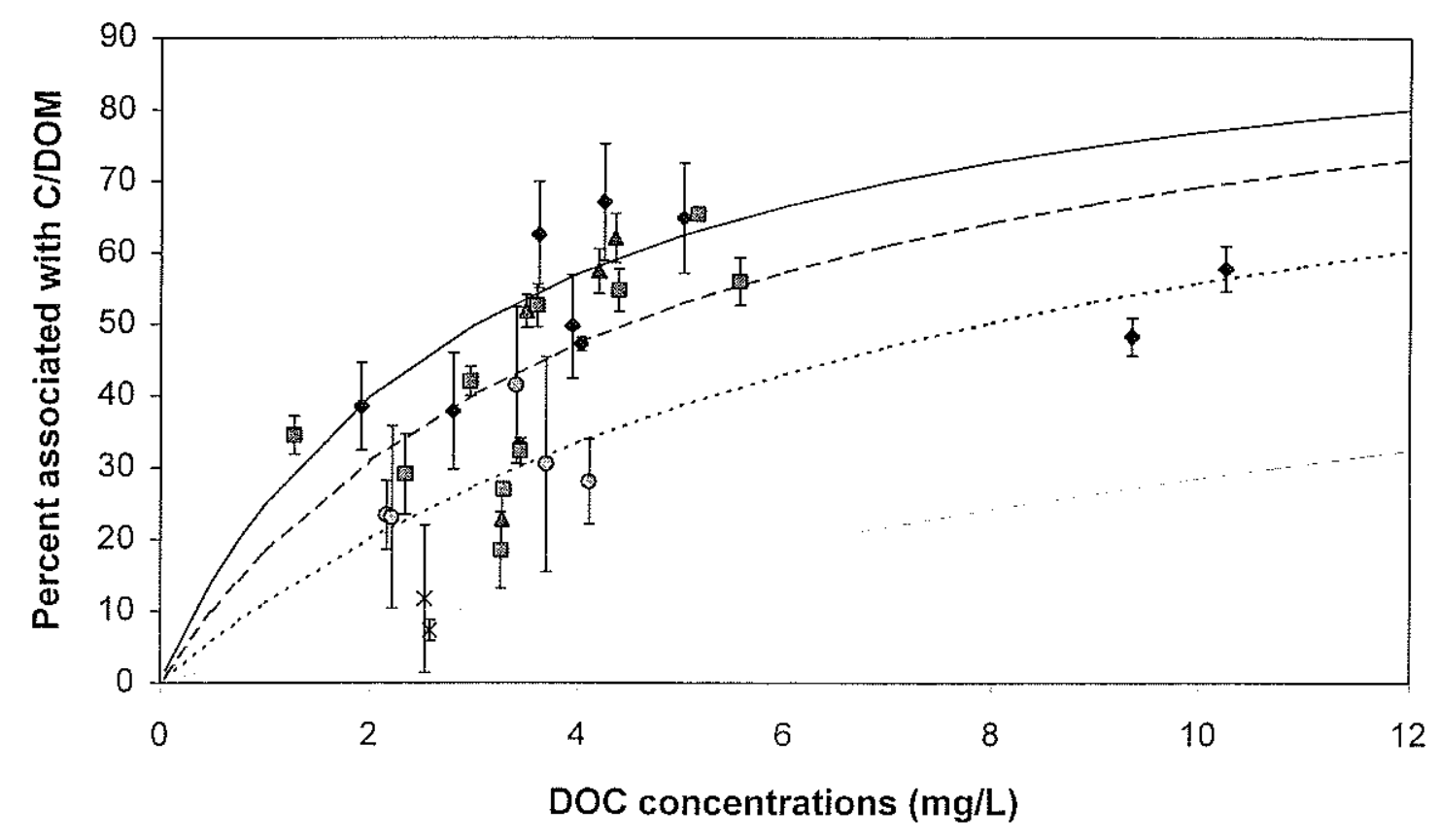

Figure 5-3. Binding data for all data versus bulk DOC concentrations with calculated $\log \mathrm{K}_{\mathrm{oc}}$. Binding data for all experiments are shown versus their respective DOC concentrations. VSW controls are represented by x's, bacterial controls (Hhalodurans) are represented by circles, Uronema are represented by diamonds, Cafeteria are represented by squares, and $P$. imperforata are represented by triangles.

Vertical error bars were propagated from errors on the average of two or three replicate headspace vessels. Lines depicting the predicted fraction bound given different $\log \mathrm{K}_{\mathrm{oc}}$ 's are also presented. The top line represents $\log \mathrm{K}_{\mathrm{oc}}=5.52$ (from P.imperforata data), the next line represents $\log \mathrm{K}_{\mathrm{oc}}=5.35$ (midway between Cafeteria and Uronema data), the next line is $\log \mathrm{K}_{\mathrm{oc}}=5.1$ (H.halodurans data) and the bottom line is $\log \mathrm{K}_{\mathrm{oc}}=4.6$ (predicted from VSW data). 


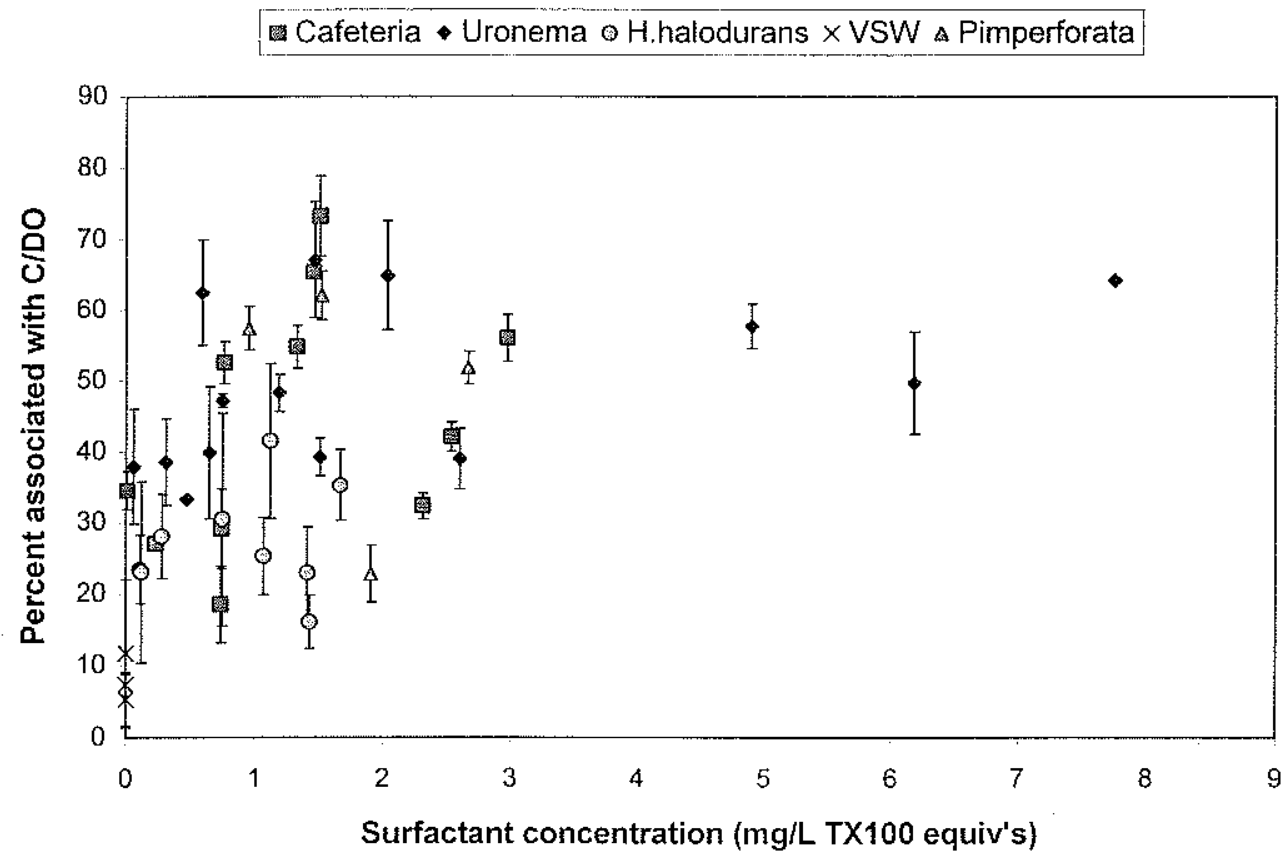

Figure 5-4. Binding data versus surfactant activity.

Data prepared in same manner as Figure 5-3.

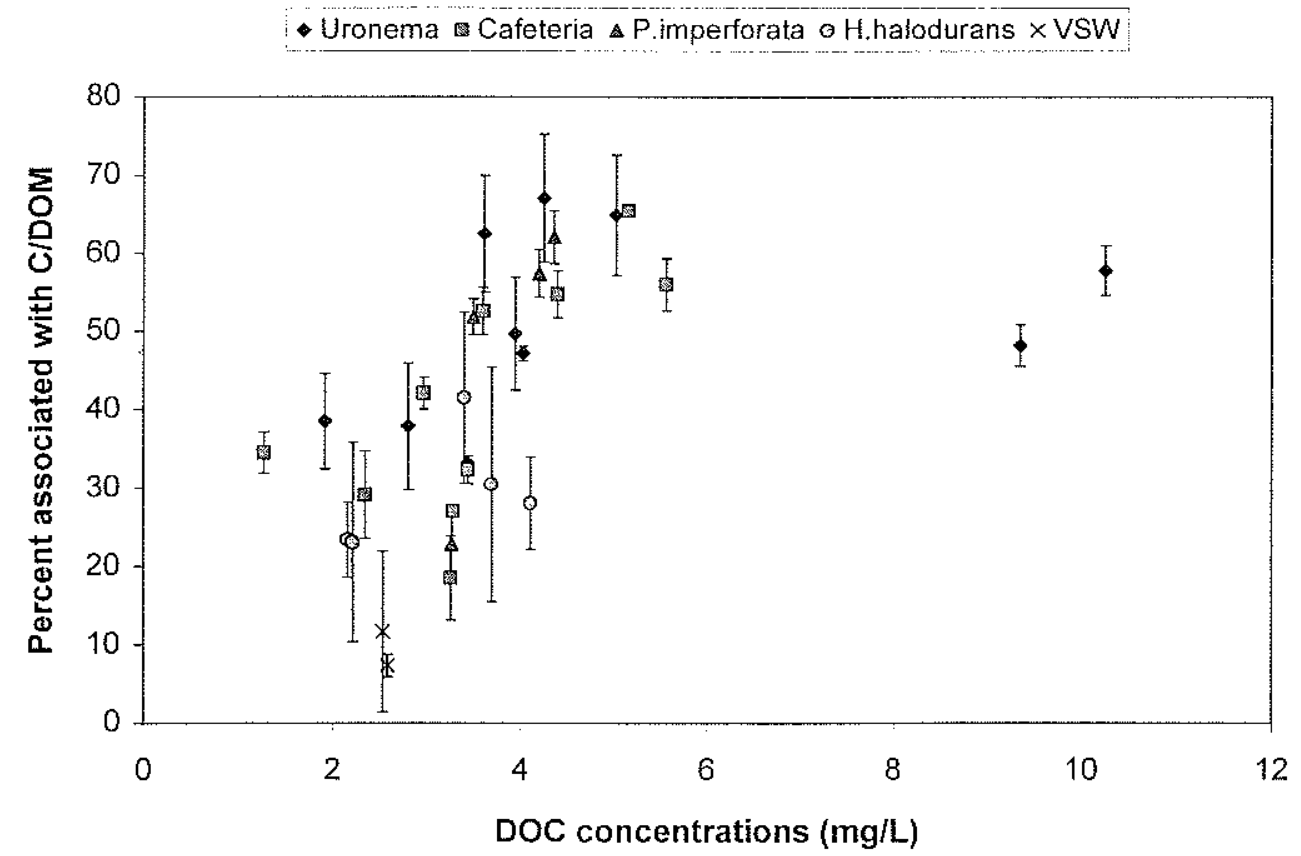

Figure 5-5. Binding data versus bulk DOC concentrations.

Same data as in Figure 5-3 except there are no lines of constant partition coefficient. 
圆 0.2u Expt 1 口0.2u Expt 2 娄 0.02u Expt 1 固0.02u Expt 2

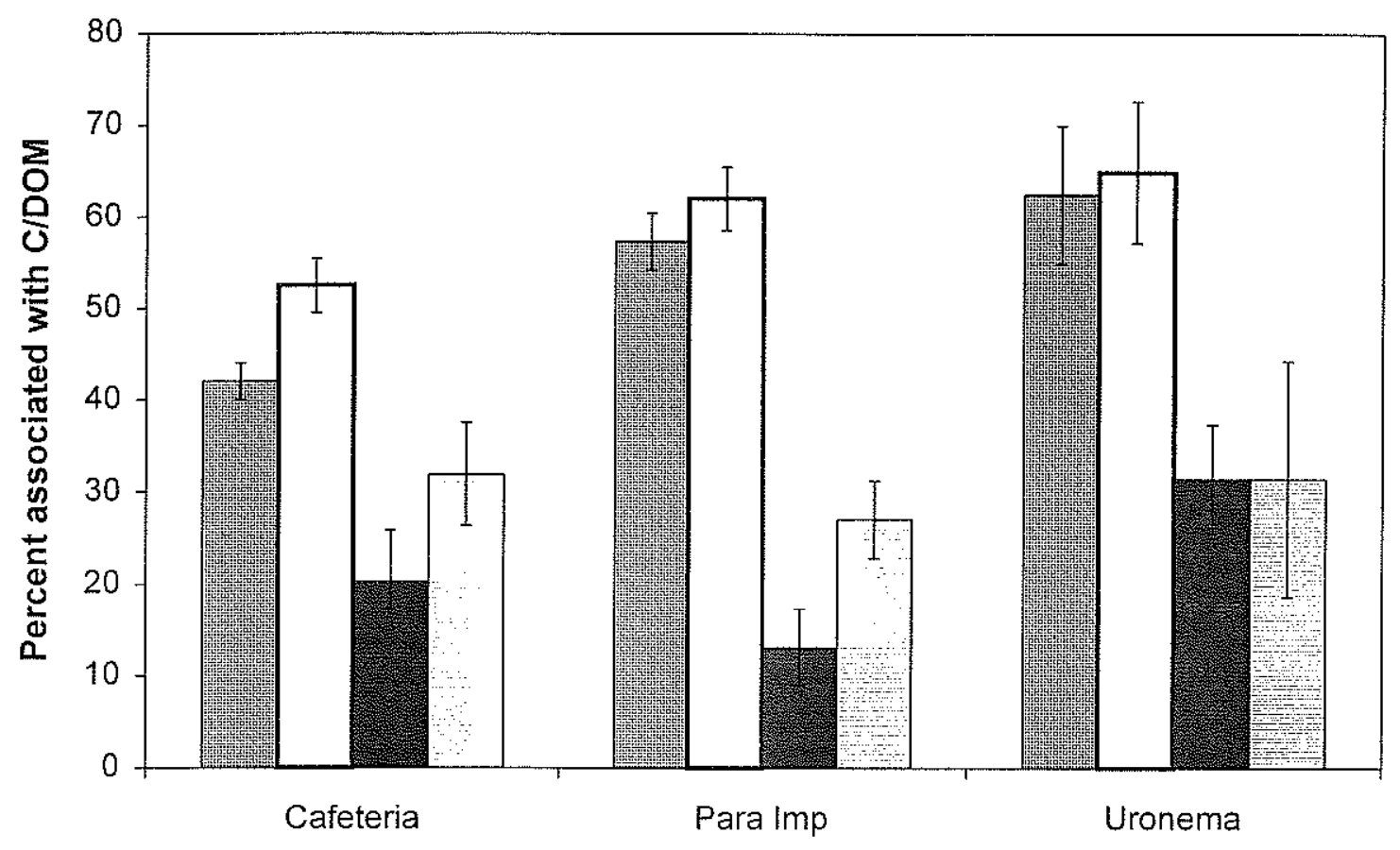

Figure 5-6. Size-fraction study.

Samples for this study were collected via $0.2 \mu \mathrm{m}$ syringe filtration. Half the sample was then filtered through $0.02 \mu \mathrm{m}$ Anopore filters. Columns shown are the average of triplicate headspace containers $\pm 1 \sigma$. 


\section{Conclusions}

\subsection{Introduction}

The over-riding goal of this thesis was to investigate the effect of protozoan grazers on the cycling of polychlorinated biphenyls (PCBs) as model hydrophobic contaminants in marine systems. Previous work in this laboratory had shown protozoan grazers were able to affect the speciation of particulate trace metals both in laboratory culture and in natural systems (Barbeau, 1998). Through intracellular digestion, protozoan grazers were able to change the chemical composition of ingested particles and reduce inorganic trace elements such as $\mathrm{Fe}^{+3}$ to biologically available forms. This thesis focused on the alteration of ingested prey and the production of dissolved organic matter with different characteristics than that seen in Vineyard Sound seawater. Since PCBs are themselves important environmental compounds of concern, elucidation of transport processes leading to remobilization of PCBs from sediments and soils is crucial to our ability to predict the long-term fate of PCBs in the natural environment and to assess remediation technologies for impacted sites.

\subsection{Summary of thesis conclusions}

\subsubsection{Chapter 2}

The work in this thesis was based on a three-phase laboratory system containing protozoan grazers, bacterial prey and dissolved organic carbon (DOC). Prior to examining the products of grazing and their impact on chlorobiphenyl (CB) speciation, it was imperative to determine the extent to which the protozoa were themselves equilibrated with PCBs in their aqueous surroundings. A theoretical calculation predicted that protozoa would accumulate PCBs via diffusion rapidly enough to be equilibrated with aqueous $\mathrm{CB}$ concentrations within minutes. This method of uptake would be significantly faster than ingestion of CB-contaminated bacterial prey. The theoretical calculation was experimentally verified with a ciliate, Uronema sp. Experimental results 
were corroborated by a numerical model comparing experimentally-derived protozoan uptake and loss rate constants and an estimated bacterial loss rate constant.

The ciliate used in initial studies was larger than other protozoa studied in subsequent chapters. Since diffusive uptake would increase in importance with increasing surface area to volume ratios, these results were applicable to the flagellates studied in following chapters. An important conclusion in this chapter was that diffusion was more important than ingestion as a mode of $\mathrm{CB}$ uptake in unicellular organisms regardless of the primary mode of nutrition - diffusive uptake or ingestion of particles and prey. Therefore, as the organism increased in size, ingestion of contaminated prey will represent an increasing fraction of the $\mathrm{CB}$ uptake. The size at which the transition from diffusion-dominated to ingestion-dominated CB uptake occurs was calculated to be 100-300 $\mu \mathrm{m}$ cell diameter.

\subsubsection{Chapter 3}

The purpose of the experiment presented in Chapter 3 was to verify the estimated bacterial loss rate constant used in the numerical model developed in Chapter 2. The extraction method chosen (sorption onto Tenax resin) was not fast enough to adequately sample the loss of PCBs from bacterial cells. Even so, the data from the Tenax experiment allowed us to set a lower limit on bacterial depuration of PCBs. To our knowledge, this was the first experimental determination of the CB bacterial loss rate constant. The data from these experiments revealed that the extraction rate constant (PCBs onto Tenax resin) in the presence of bacterial cells and dissolved organic matter (DOC) was faster than predicted from the control extraction rate constant and the estimated aqueous $\mathrm{CB}$ fraction. The enhanced extraction rate constant was shown to be the product of DOC-enhanced diffusion analogous to reaction-enhanced transport of $\mathrm{CO}_{2}$ across the air-water interface. Interactions between aqueous PCBs and bacterial-derived DOC increased the amount of PCBs available for resin extraction on short time scales. The increased diffusion resulted in faster equilibration than anticipated, but was not expected to alter the thermodynamically predicted equilibrium CB concentrations in the 
organic pools of the system. Preliminary extensions to other congeners suggest that this phenomenon will be present for all congeners studied. The effect of DOC-enhanced diffusion has implications for the prediction of $\mathrm{CB}$ accumulation by organisms within a certain period of time. In systems with kinetic barriers to full equilibration, DOCenhanced diffusion may decrease the time needed to achieve equilibrium. Thus simple calculations using uptake rate constants for pure particles or unicellular organisms may underestimate the diffusive uptake rate and, in turn, the CB accumulation in an organism over a particular exposure time.

\subsubsection{Chapter 4}

Chapters 2 and 3 showed that the time scale for equilibration of the organisms studied was minutes to seconds. The time scale of grazing processes and production of dissolved material was anticipated to be hours to days. Therefore, full CB equilibration was assumed in the remaining studies and the focus of the thesis turned to the products of grazing and their role in $\mathrm{CB}$ speciation. The nature of the dissolved organic material produced by different protozoan grazers was the focus of the experiments described in Chapter 4. Bulk dissolved organic carbon (DOC), surfactants and lipid material were all monitored in grazing cultures of a ciliate, Uronema sp., and two flagellates, Cafeteria sp. and Paraphysomonas imperforata. These experiments showed that bulk DOC cycles were not predictive of specific sub-pools such as surface-active material or lipopolysaccharides. Lipid material was a small fraction of the total DOC $(<10 \%)$ and was relatively constant across the protozoan species studied and bacterial controls. Limited compositional highlighted the lack of phospholipid-rich material whose presence had been predicted by Nagata and Kirchman (1992b).

Interspecies differences were noted in the production of surfactant material, with the ciliate producing significantly more surface-active material than either of the two flagellates. This is the first quantitative assessment of surfactant production over time in heterotrophic protozoan cultures. Circumstantial evidence had previously implicated protozoa, especially ciliates, in surfactant production (Barbeau, 1998). The magnitude of 
the production observed here is comparable to or higher than phytoplankton production noted in other studies (Zutic et al., 1981; Frew et al., 1990). The controlling factors for surfactant production are a complex mixture of protozoan feeding mechanism, digestive chemistry and prey surface and cellular composition. Further work is needed to isolate and study each of these factors and their respective effects on surfactant production and composition.

\subsubsection{Chapter 5}

The fifth and final data chapter focused on the affinity of "grazer-enhanced" DOM for PCBs relative to bacterial-derived DOM and background Vineyard Sound seawater (VSW) organic matter. Affinities were measured with headspace partitioning experiments. Equilibrium partitioning coefficients were calculated for each culture and significant differences were noted among the three types of filtrates studied. The $\mathrm{K}_{\mathrm{DOC}}$ 's were highest for protozoan culture filtrates $\left(10^{5.35}\right)$, intermediate for bacterial culture filtrates $\left(10^{5.1}\right)$, and lowest for VSW controls $\left(10^{4.6}\right)$. The high partition coefficients observed in the protozoan culture filtrates could be due either to high concentrations of low-affinity material or low concentrations of very-high affinity material. Estimates of the amount of "pure" lipid material that would be needed to generate the binding data observed were comparable to or higher than lipid concentrations measured by $\mathrm{CHCl}_{3}: \mathrm{MeOH}$ extraction (Chapter 4). The fact that the quantity of lipid material present could not be used to explain the observed binding in the culture filtrates suggests that other "grazer-enhanced" DOM was playing a significant role in binding PCBs. The nature of this binding, be it structurally or compositionally-driven, is not clear at this time. The partition coefficients calculated were relatively species-independent as well as growth-substrate independent. This suggests that enhanced binding affinity is not dependent on a particular protistan species. 


\subsection{Thesis implications}

\subsubsection{Equilibrium dynamics in the microbial loop}

Recent laboratory studies have suggested that growth and the subsequent dilution of PCBs by new biomass prevent certain organisms from achieving full equilibration with aqueous PCBs (Swackhamer and Skoglund, 1993; Skoglund et al., 1996). Field studies have also observed apparently non-equilibrated size fractions $(>20 \mu \mathrm{m})$ in marine systems (Axelman et al., 1997). These investigators have suggested that some classes of unicellular organisms are not fully equilibrated with aqueous PCBs and care must be taken when assessing the amount of PCBs that are introduced into the marine food chain through diffusive uptake into the unicellular organisms encompassing the lowest tier. The data in Chapters 2 and 3, on the other hand, are consistent with the idea that organisms in the microbial loop are in full equilibrium with their surroundings. There are a number of issues that must be addressed when examining these seemingly conflicting studies. First, the same size organisms must be compared. Our data is consistent with the field data of Axelman et al. (1997) which showed that organisms in the 2-20 $\mu$ m size fraction were equilibrated with aqueous $\mathrm{CB}$ concentrations. The laboratory study by Swackhamer et al. examined CB uptake in algae ranging from $20-30 \mu \mathrm{m}$ in diameter.

Second, DOC concentrations must be measured and considered. Studies that use $0.2 \mu \mathrm{m}$ filtration to define "aqueous" PCBs without a correction for dissolved organic matter will necessarily overestimate the amount of PCBs available for diffusive uptake into organisms. High DOC concentrations will enhance the diffusive uptake rate of lower-chlorinated congeners such that the kinetic barrier represented by the "sequestration" of PCBs by DOC will not be observed, regardless of congener hydrophobicity. Accurate determination of the truly dissolved aqueous CB concentration is extremely difficult due to the particle-reactivity and low aqueous concentrations of these compounds (Schulz-Bull et al., 1991). However, preliminary estimates of the aqueous concentration can be made if the organic carbon content of the different pools is known. Axelman et al. (1997) note the difficulty of constraining the aqueous CB concentration in their field study. 
Third, one must take care to understand the basis of the equilibrium that is being discussed. As shown in Chapter 5, equilibrium partition coefficients vary one or two orders of magnitude depending on the chemical nature of the material involved. It is imperative to use the proper equilibrium partition coefficient when assessing the extent to which an organism has equilibrated with its surroundings. For example, Swackhamer $e t$ al. (1993) normalize particulate CB concentrations to cellular lipid content. However, assessment of the extent to which organisms have equilibrated is based on the change in $\mathrm{CB}$ concentrations in the particulate phase (as a whole) over time as well as the fraction in the aqueous phase. The time course of the experiment described in Swackhamer et al. (1993) was long enough for the phytoplankton to increase in size and numbers. Changes in phytoplankton composition over time will affect the equilibrium concentration as well as the extent to which the organism is equilibrated. Equilibration with all organisms within the microbial loop should be considered when estimating the quantity of PCBs entering the food chain in aquatic environments.

\subsubsection{Production of heterogenous C/DOM}

Numerous studies have attempted to quantify and characterize the production of dissolved organic matter by the microbial loop in natural systems (Caron et al., 1985; Sherr and Sherr, 1988; Jumars et al., 1989; Nagata and Kirchman, 1992b; Nagata and Kirchman, 1992a; Tranvik, 1994; Pelegri et al., 1999). The different protozoan and prey species used make direct comparisons between the various studies difficult. However, it is clear that protists can generate large quantities of "grazer-enhanced" DOM through the grazing process. The chemical composition of this material is under investigation but seems to be a function of the digestive cycle of the protist and the chemical composition of the prey. Results from this thesis suggest that at least a portion of this material has surface-active properties. Lipid concentrations were consistent with previous work (Nagata and Kirchman, 1992b).

Specific composition of the material has been measured indirectly either by radioactive labeling of the bacterial prey (Tranvik, 1994) or by enzyme studies (Nagata 
and Kirchman, 1992b). These studies have proposed that colloidal/dissolved organic material was comprised of internal cellular components and non-cell wall material. This material must be rather labile because high concentrations of lipid and cellular material are not observed in the ocean (Aluwihare et al., 1997). The lack of naturally-occurring material of this nature in the ocean may point to relatively short residence times. Short residence times and relatively high affinity of this material for PCBs may explain the field observations of Baker et al. (1991) and Sanders et al. (1996) that PCBs are preferentially remobilized at the sediment-water interface relative to organic carbon.

\subsubsection{Applicability to natural systems}

The results of this thesis are most applicable to environments of high microbial loop activity - including sediment-water interfaces of coastal areas and lakes, groundwater aquifers, and sewage sludge disposal areas. Because protozoa are aerobic organisms, the activities described herein would be confined to oxic zones. These sites have high nutrient concentrations which support dense bacterial populations. The presence of protozoa in many of these sites has been used to explain increased degradation and remineralization of organic matter (Sinclair et al., 1993; Madsen et al., 1996). Protozoa themselves have not been implicated in the remineralization of organic

matter. Rather, their grazing pressure keeps bacterial populations growing at exponential rates even though the size of the population is not increasing (Shen et al., 1986). Bacterial growth stage has been shown to affect the degree of remineralization in that higher rates of degradation are observed during exponential growth. From this thesis, it is clear that protozoa will be instrumental in changing the chemical composition of the organic matter, not only with grazing pressure, but also with the excretion of partiallydigested bacterial cells. The presence of lipid material and amphiphilic surfactants will only increase the affinity of "grazer-enhanced" DOM for hydrophobic organic contaminants such as PCBs. High production rates of surfactant material were noted at the transition from exponential to stationary growth in the protozoan population- 
suggesting that healthy protists are necessary for surfactant production but not necessarily those in exponential growth.

In heavily impacted sites like New Bedford Harbor, hydrophobic organic contaminants such as PCBs are mixed with heavy metals such as zinc, silver and cadmium. High concentrations of these metals have been shown to be toxic to some protozoan species (Madoni et al., 1994). Early in my thesis work, I collected samples from the sediment-water interface in New Bedford Harbor. With the help of Dave Caron, several protozoan species were observed and isolated. These species included nanoflagellates, hymenociliates, scuticociliates, hypotrichs and amoebas. The diversity of the protozoan assemblage strengthens the applicability of this thesis work to natural regimes. The protists I studied exhibited no signs of a toxic response to CB exposure, i.e., no change in growth rate or morphology was observed. However, if the presence of heavy metals (as in New Bedford Harbor) were to sharply decrease the diversity of the protozoan assemblage, the results from this study would have to be tempered by concerns over heavy metal toxicity in extreme environments. This issue is discussed in detail in Pratt and Cairns (1985).

The largest source of PCBs to coastal waters in urban areas is contaminated sediments. Remobilization of inert PCBs from these systems during organic carbon diagenesis is an important process to consider and quantify. Field studies have shown that remobilization of PCBs from sinking particulate material increases the residence time of PCBs in lakes (Baker et al., 1991; Sanders et al., 1996). In impacted coastal areas and estuaries, many PCBs are associated with high concentrations of sediment organic matter. During diagenesis and release of organic matter to pore-waters, PCBs will also be remobilized and be associated with pore-water DOC. When pore-waters are flushed, these PCBs will be released into the overlying sediment-water interface and will re-enter the dynamic DOC system of the microbial loop. Once these contaminants are back in the sediment-water interface and are participants in the organic carbon cycling loop, they can be easily transported back into the overlying water column and other areas 
through water mass transport. In this way, the microbial loop can contribute to the transport of PCBs out of contaminated sediments.

These results are not easily extended to polynuclear aromatic hydrocarbons (PAHs). Sanders et al. (1996) noted that the depositional flux and burial rate of PAHs were equivalent. They proposed that PAHs were sequestered in a non-exchangeable pool of organic matter. These results are consistent with recent studies involving soot carbon (McGroddy and Farrington, 1995; Gustafsson et al., 1997). Soot carbon is formed during the combustion of fossil fuels - much like many of the PAHs. PAHs are then trapped within the matrix of the soot particle. In addition, this substrate has a very high affinity for PAHs (Gustafsson et al., 1997) and so is a near-irreversible sink for PAHs in some areas. The fate of soot particles within the gut of protists is unknown. It is not known whether protozoan digestive enzymes could degrade this recalcitrant matrix made of nearly pure carbon.

\subsection{Future work}

As with any scientific endeavor, this thesis has raised a number of questions that should be addressed by future investigators. From Chapters 2 and 3, more work is needed to ascertain experimentally the transition between diffusion-dominated and -ingestion-dominated CB uptake, taking into account morphological features such as cilia, frustules and reticulopodia. The uptake rate constants for large phytoplankton such as large diatoms will be crucial parameters in estimating the time needed to achieve equilibrium within a particular aquatic environment and in turn quantifying the amount of PCBs that are available for transport into the aquatic food chain. These rate constants can have implications for the overall "bioavailability" of PCBs within a system.

Clearly the role of DOC in enhancing diffusive uptake should be further investigated. To that end, specific components of this material need to be characterized and their affinities for PCBs should be quantified, both in isolation and in concert. Control experiments with known solutions of biological compounds such as albumin, cellulose, lipopolysaccharide, and sterols, can be used to standardize the $\mathrm{CB}$ affinities 
generated by the headspace partitioning technique. For example, headspace experiments with cellulose should be compared to previous sorption studies by Garbarini and Lion (1986). In addition, fluorescent studies such as those in Backhus et al. (1990) or structural studies such as those in Bortiatynski et al. (1997) could be employed to characterize the binding environment of the material produced during grazing. Hydrodynamic fractionation of this material by techniques such as SPLITT fractionation (Keil et al., 1994) could be used to quantify the colloidal fraction in terms of settling potential as suggested by Gustafsson and Gschwend (1997). In short, the basis of the increased binding observed in "grazer-enhanced" DOM should be examined. With reliable estimates for the physico-chemical properties of this material, reasonable predictions for the transport of PCBs out of contaminated sediments could be determined.

In concert with the physico-chemical studies, further compositional studies of "grazer-enhanced" DOM are needed. The influences of feeding mechanism, digestive chemistry and prey species are particularly important factors to elucidate. The components of "grazer-enhanced" DOM are a direct consequence of the digestive efficiency of the protozoan species studied. Radio-labeled prey have been used somewhat effectively to track bacterial components as they are digested and then excreted as organic matter (Tranvik, 1994). However, these studies have only been able to distinguish between internal and external cellular components. More specific compositional work such as specific binding probes or structural studies (e.g., Aluwihare, 1997) is needed to determine the particular chemical composition of this material. The internal composition of the prey species must be considered in all these studies and so DOM produced in prey controls should be examined. Since the CB affinity of organic matter is a function of both composition and physico-chemical properties, the composition of this material should be determined to get a complete picture of the impact on $\mathrm{CB}$ speciation in a particular environment.

Lastly, all of this work should be confirmed with field studies of contaminated environments. The composition of organic material at the sediment-water interface should be compared to that generated in grazing laboratory cultures. For example, 
Barbeau and Moffett (Barbeau and Moffett, submitted) found that dissolution of iron oxyhydroxides in field samples was in the same range as those measured in the laboratory. The binding affinity of fresh organic matter should be compared to that of "grazer-enhanced" DOM. These studies should encompass all environments where microbial processes are important including coastal areas, estuaries, groundwater aquifers and lake sediment-water interfaces.

This thesis has shown that protozoan grazers can play an important role in the cycling of PCBs in contaminated environments by producing DOM with relatively high affinities for PCBs. The material produced as a result of protozoan grazing is short-lived and has an enhanced affinity for PCBs over background seawater DOC. This thesis is the first indication that production of DOM through grazing can contribute to the transport of PCBs from contaminated sediments and result in longer residence times of PCBs in the water column. Further laboratory and field studies are needed to quantify the flux of PCBs out of contaminated soils and sediments and to characterize the components of DOM that are most responsible for this flux. 


\section{Appendix A - Effect of boundary layer on diffusive uptake of PCBs by protozoan cell}

The flux through the stagnant water boundary layer surrounding the protozoan cell can be described by Fick's law of diffusion:

$$
\text { (1) } F l u x=-D \frac{\Delta C}{\Delta z}=-D_{w} \frac{C_{w}-C_{w / c}}{z_{w}}
$$

where $\mathrm{D}_{\mathrm{w}}$ is the molecular diffusion coefficient of the diffusant (in this case, $C B$ congener) through water $\left(\mathrm{m}^{2} / \mathrm{sec}\right), \mathrm{C}_{\mathrm{w}}$ and $\mathrm{C}_{\mathrm{w} / \mathrm{c}}$ are the concentrations of the diffusant in the bulk water and at the water/cell interface, respectively $\left(\mathrm{g} / \mathrm{m}^{3}\right)$ and $\mathrm{z}_{\mathrm{W}}$ is the width of the boundary layer $(\mathrm{m})$. The concentrations referred to in this equation are the truly dissolved concentrations and so they can both be re-written in terms of the total $[\mathrm{CB}]_{\text {Tot }}$ concentration and the dissolved organic carbon-associated concentration ([CB] $]_{\text {org }}$ ) using the organic carbon-water partition coefficient, $\mathrm{K}_{\mathrm{oc}}$. In the boundary layer and in the bulk solution, the organic carbon in association with the PCBs is dissolved organic carbon (DOC) and at the cell surface, the organic carbon in question is lipid material within the cellular membrane. First, the total CB concentration is the sum of the organic carbonassociated PCBs and the truly dissolved, or aqueous, PCBs ([CB $\left.]_{\mathrm{aq}}\right)$.

$$
\text { (2) }[C B]_{T o t}=[C B]_{o r g}+[C B]_{t a q}
$$

The organic carbon-associated PCBs and the aqueous PCBs are related to one another through the organic carbon-water partition coefficient.

$$
\text { (3) } K_{u c}=\frac{[C B]_{o r g} /[\text { org }]}{[C B]_{c t}}
$$

where [org] is the organic carbon concentration $\left(\mathrm{g} \mathrm{OC}^{3} \mathrm{~m}^{3}\right)$. These two equations can be combined for the bulk solution and for the water/cell interface to give $\mathrm{C}_{\mathrm{w}}$ and $\mathrm{C}_{\mathrm{w} / \mathrm{c}}$.

$$
\begin{aligned}
& \text { (4) } C_{w}=\frac{[C B]_{\text {rot }}}{1+K_{w c}[\text { org] }} \\
& \text { (5) } C_{w / c}=\frac{[C B]_{T w}}{1+K_{l i p}[\text { lip }]}
\end{aligned}
$$


where $K_{\text {lip }}$ is the lipid-water partition coefficient and [lip] is the lipid concentration in the cell surface. These equations are then substituted into the flux equation above.

$$
\text { (6) } F l u x=-D_{w} \frac{[C B]_{h o s}}{z_{w}}\left(\frac{1}{1+K_{o c}[O C]}-\frac{1}{1+K_{\text {lip }}[\text { lip }]}\right)
$$

To simplify this equation, I assumed that the second term was approximately zero. The first term is equal to 0.8 when $\mathrm{K}_{\mathrm{oc}}=10^{5.4}$ (from relationship in Schwarzenbach et al. $(1993)$ ) and $[\mathrm{OC}]=5 \mathrm{mg} / \mathrm{L}$ or $5 \times 10^{-6} \mathrm{~g} / \mathrm{mL}$. In the second term, $\mathrm{K}_{\text {lip }}=10^{6.33}$. As long as [lip] is greater than $50 \mathrm{mg} / \mathrm{L}$, the simplification will be valid. The simplified equation is:

$$
\text { (7) Flux }=-D_{w} \frac{[C B]_{T o t}}{z_{w}}(0.8)
$$

$\mathrm{D}_{\mathrm{w}}$ can be estimated from the relationship in Schwarzenbach et al. (1993).

$$
\text { (8) }\left(\frac{D_{w}(\text { unknown })}{D_{w}(\text { known })}\right)=\left(\frac{M W(\text { known })}{M W(\text { unknown })}\right)^{0.5}
$$

For the purposes of this calculation, the known compound is oxygen gas $\left(\mathrm{O}_{2}\right)$ whose molecular diffusion coefficient is $2.1 \times 10^{-5} \mathrm{~cm}^{2} / \mathrm{s}$ and molecular weight is $32 . \mathrm{D}_{\mathrm{w}}$ for congener \#77 (MW=290) was calculated to be $6.98 \times 10^{-6} \mathrm{~cm}^{2} / \mathrm{s}$ or $6.98 \times 10^{-10} \mathrm{~m}^{2} / \mathrm{s}$. Substituting this value into the simplified flux equation, I get:

$$
\text { (9) Flux }=-5.58 \times 10^{-10} \frac{[C B]_{T h t}}{z_{w}}
$$

In the case of no boundary layer (as in the introduction of Chapter 2), I estimated that the rate of diffusive uptake would be $3.3 \times 10^{4}$ times that of the rate of ingested uptake. The rate equation for diffusive uptake can be written as:

$$
\left(\frac{d[C B]_{p r o t}}{d t}\right)_{d i f f}=F l u x * S A_{p r o t}=\frac{D_{m} K_{l w} S A_{p r o t}}{\Delta z}[C B]_{d}
$$

Using the values for $\mathrm{D}_{\mathrm{m}}, \mathrm{K}_{\mathrm{iw}}$ and $\Delta \mathrm{z}$ from Chapter $2, \mathrm{I}$ get the following simplification of the above equation: 


$$
\text { (11) Diff }=\left(\frac{d[C B]_{\text {prot }}}{d t}\right)_{\text {diff }}=0.02 * S A_{\text {prot }} *[C B]_{d}
$$

In Chapter 2, the ratio of diffusive uptake rate to ingested uptake rate was $3.3 \times 10^{4}$. Therefore:

$$
\text { (12) Ing }=\frac{\text { Diff }}{3.3 \times 10^{4}}=\frac{0.02 * S A_{p r o t} *[C B]_{d}}{3.3 \times 10^{4}}=6.1 \times 10^{-7} * S A_{p r o t} *[C B]_{d}
$$

I substituted the equation 4 for $[\mathrm{CB}]_{\mathrm{d}}$ to get:

$$
\text { Ing }=6.1 \times 10^{-7} * S A_{p r o t} *\left(\frac{[C B]_{T o t}}{1+K_{o c}[O C]}\right)=4.9 \times 10^{-7} * S A_{p r o t} *[C B]_{T o t}
$$

To get an estimate for the boundary layer thickness that would impede diffusive uptake, I set the above formulation for ingested uptake rate (Equation 13) equal to diffusive uptake through the stagnant water boundary layer (Equation 11).

$$
5.6 \times 10^{-10} \frac{[C B]_{\text {Yot }}}{z_{w}} S A_{p r o t}=4.9 \times 10^{-7}[C B]_{T y t} S A_{p r o t}
$$

The terms $[\mathrm{CB}]_{\mathrm{Tot}}$ and $\mathrm{SA}_{\mathrm{prot}}$ cancel and $\mathrm{z}_{\mathrm{w}}$ is equivalent to $1.1 \mathrm{X} 10^{-3} \mathrm{~m}$ or $1100 \mu \mathrm{m}$. 


\section{Appendix $B-$ Raw data for Chapter 2 bioaccumulation experiment}

The masses of each CB congener in each sample are given. The mass of each congener was calculated once using the percent recovery of \#14 and once using the percent recovery of \#198. These two values were then averaged. The averages and standard deviations (in italics) are presented above. The $\log \mathrm{K}_{\text {ow }}$ values were taken from Hawker \& Connell (1988). The percent recoveries of \#14 and \#198 are also given. In some aqueous samples, the iso-octane layer containing the internal recovery standards was lost prior to extraction. The chromatograms showed that the internal recovery standards were abnormally low relative to the $\mathrm{CB}$ spike masses. In these cases, the recovery for the $\mathrm{CB}$ spike was assumed to be $100 \%$.

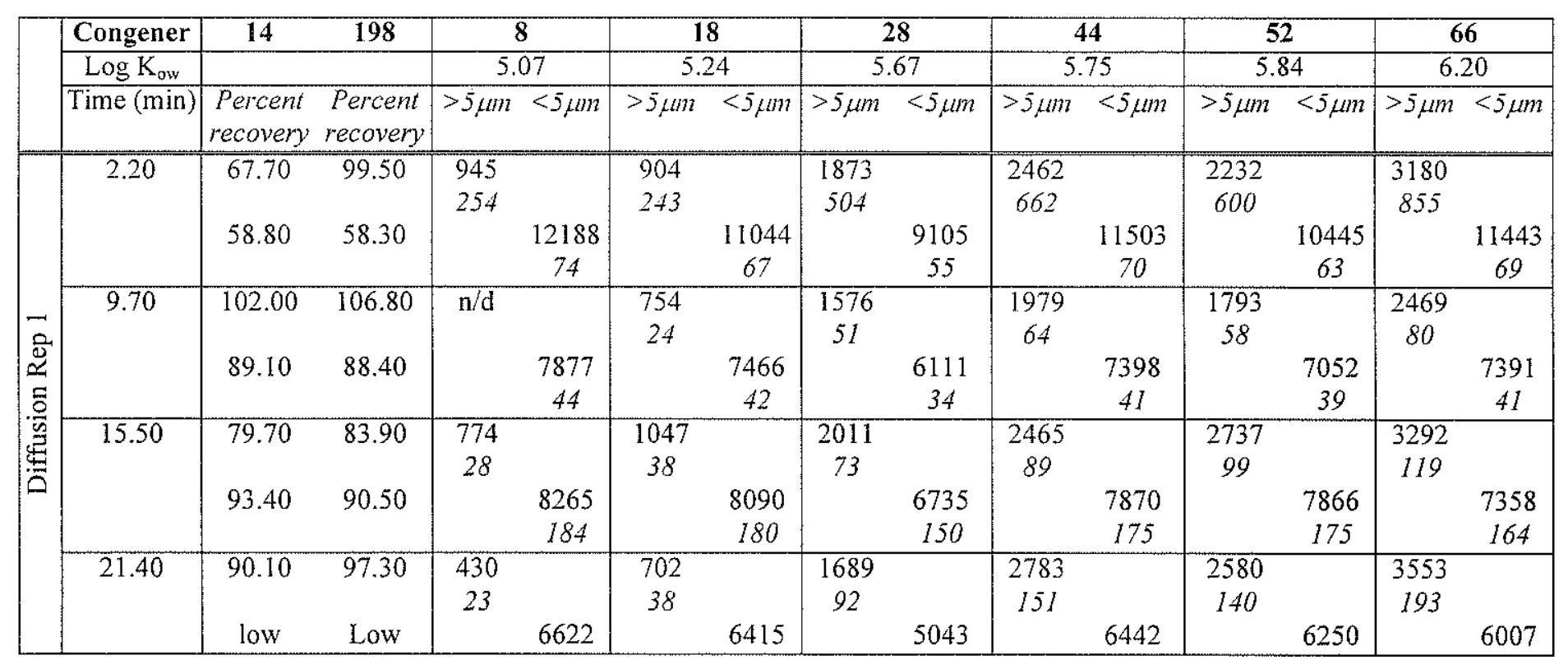




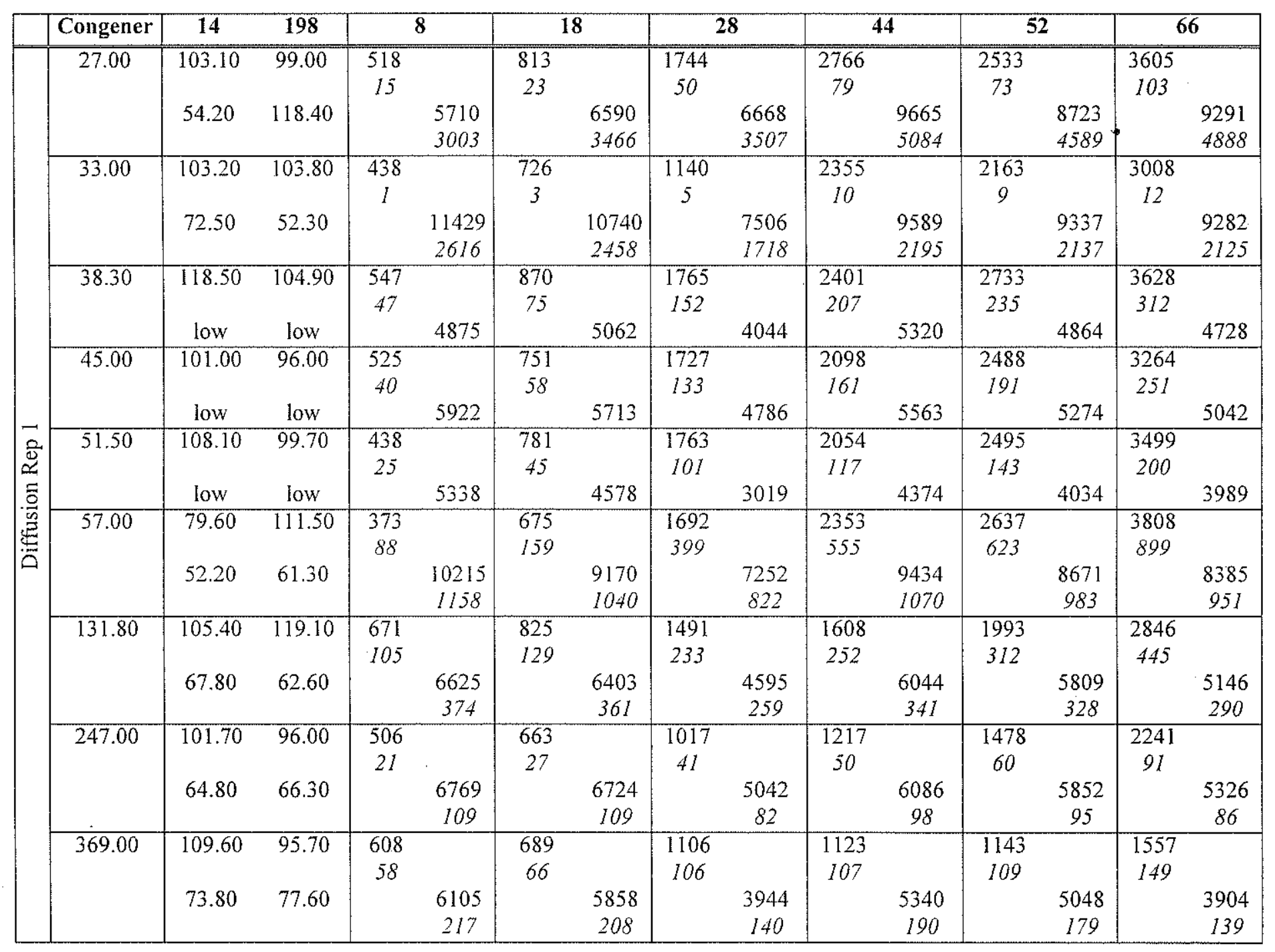




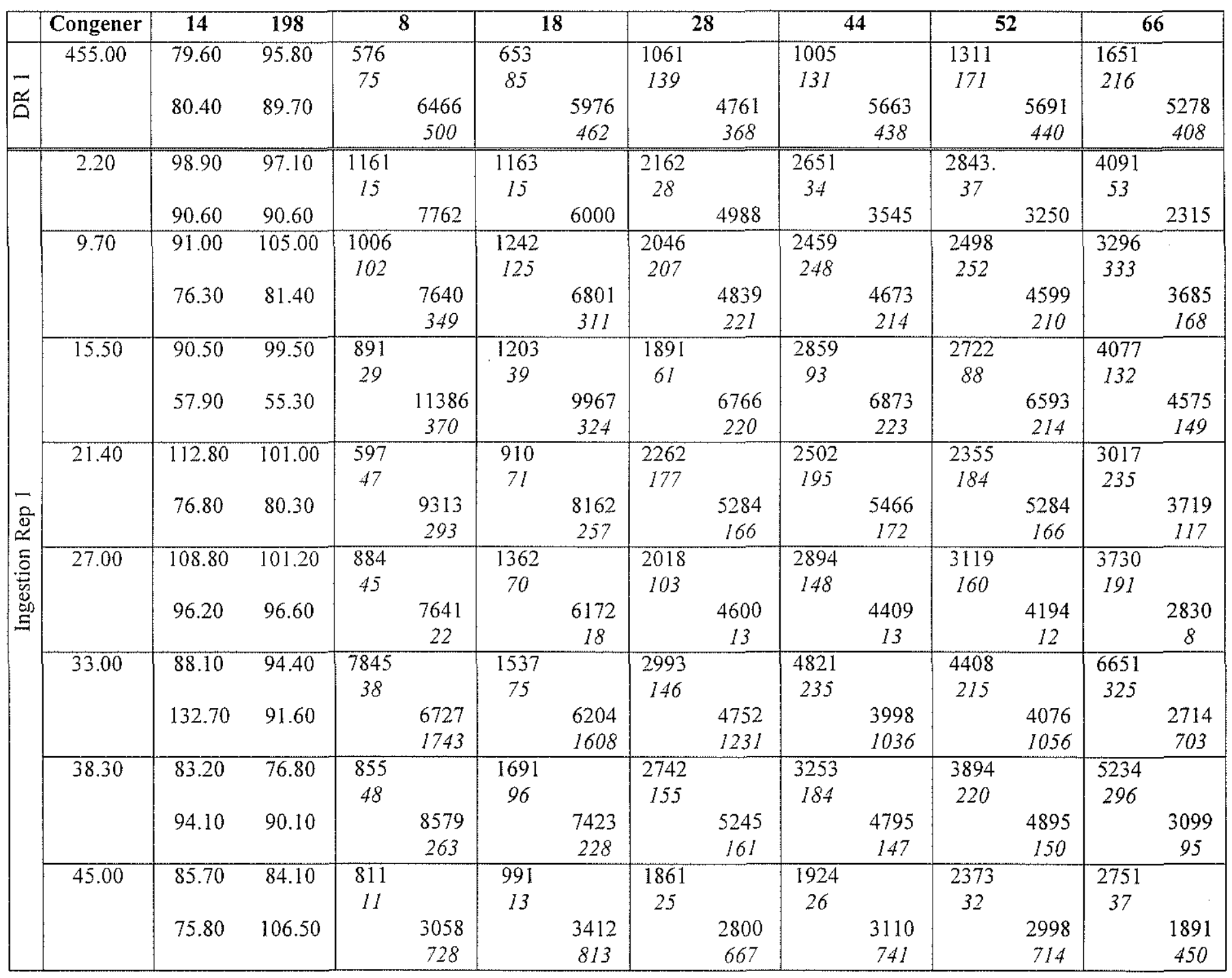




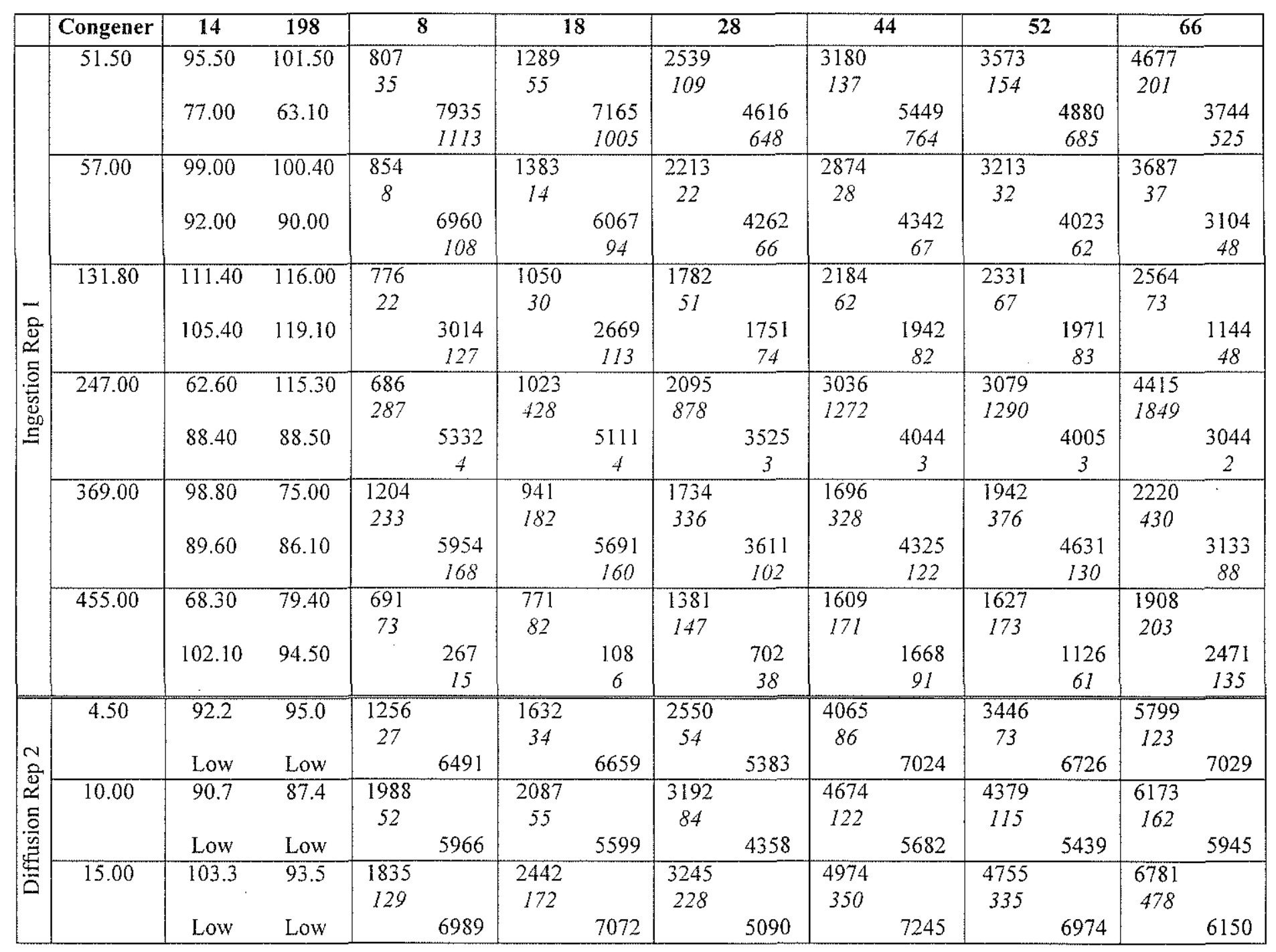




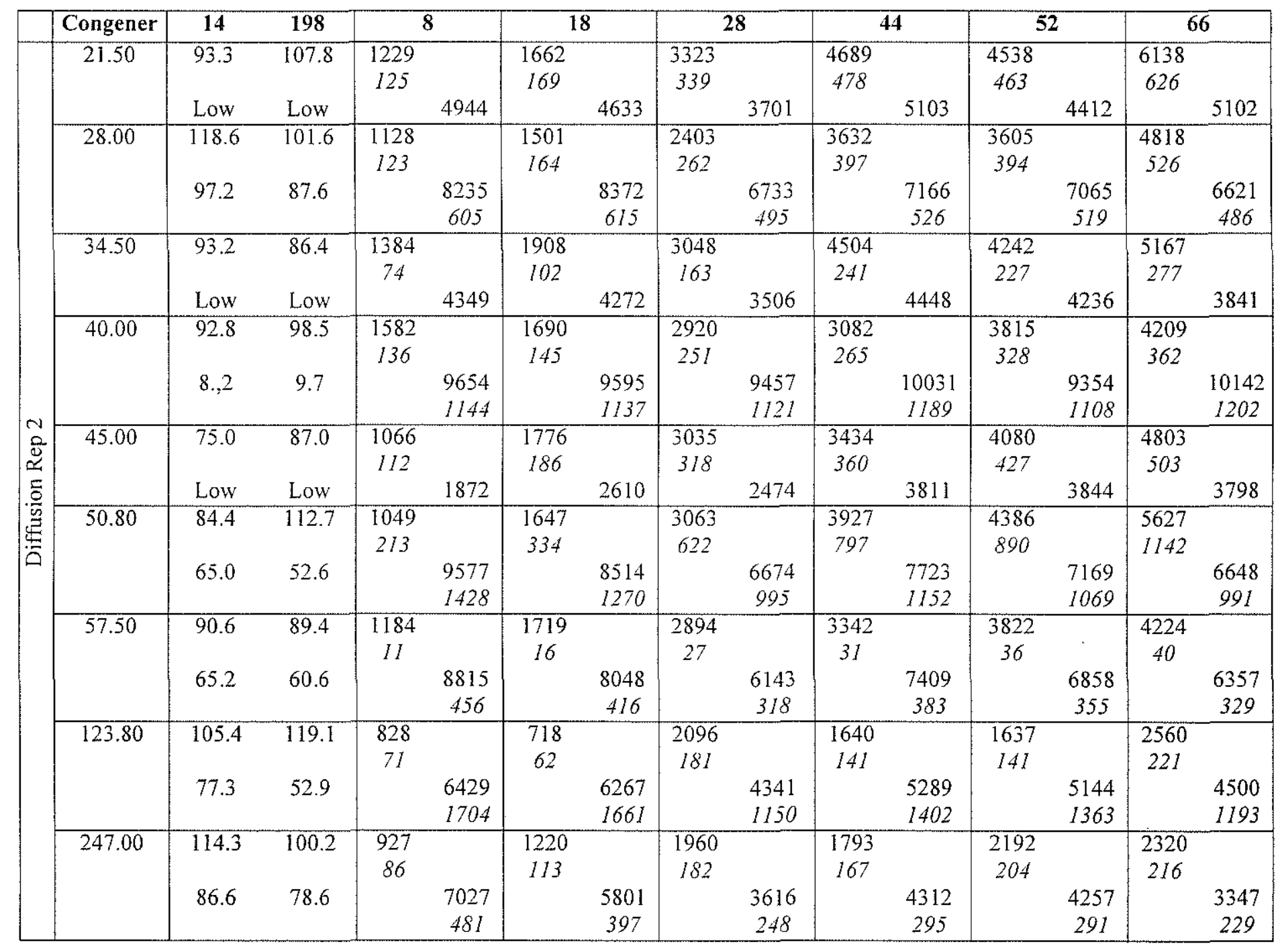




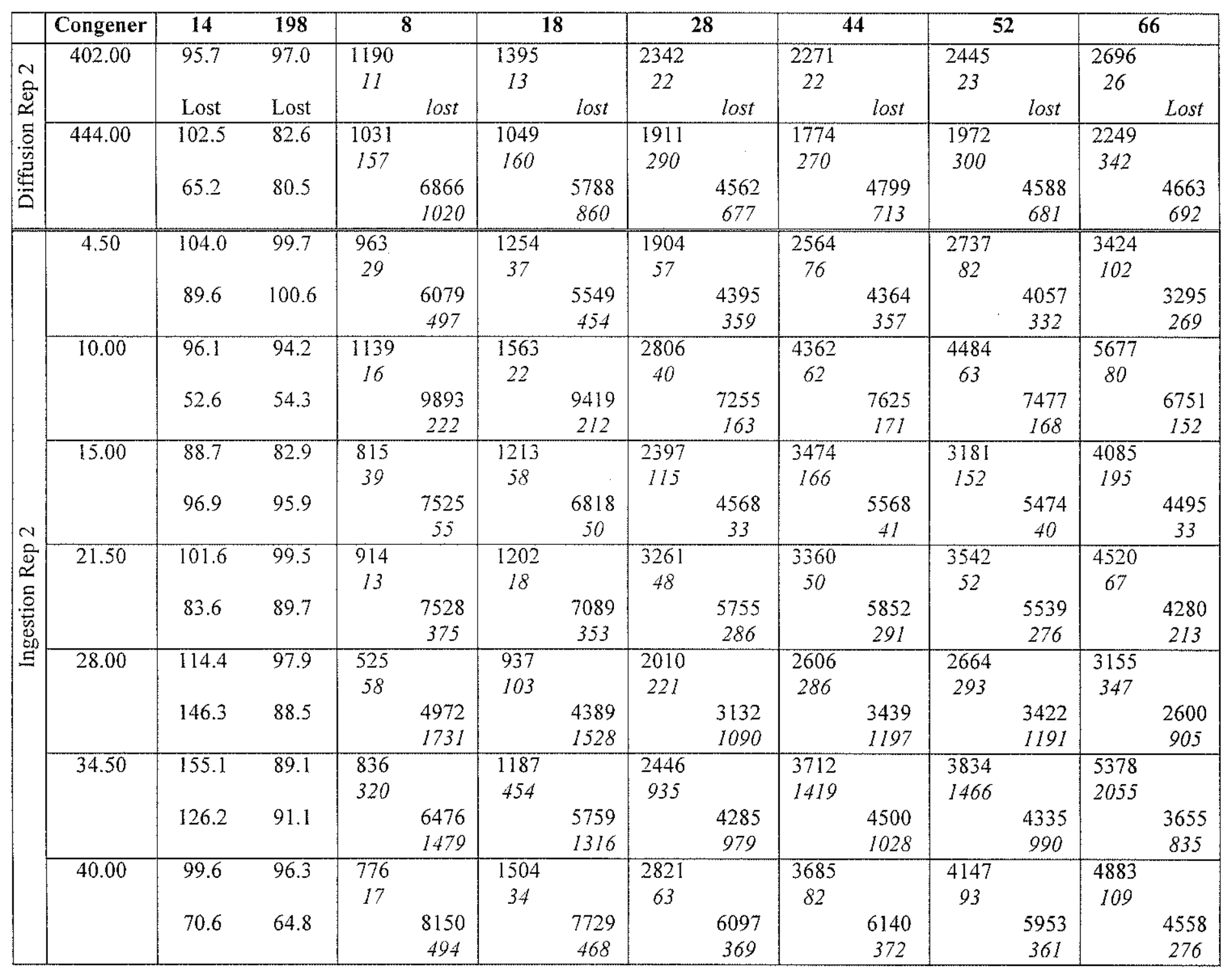




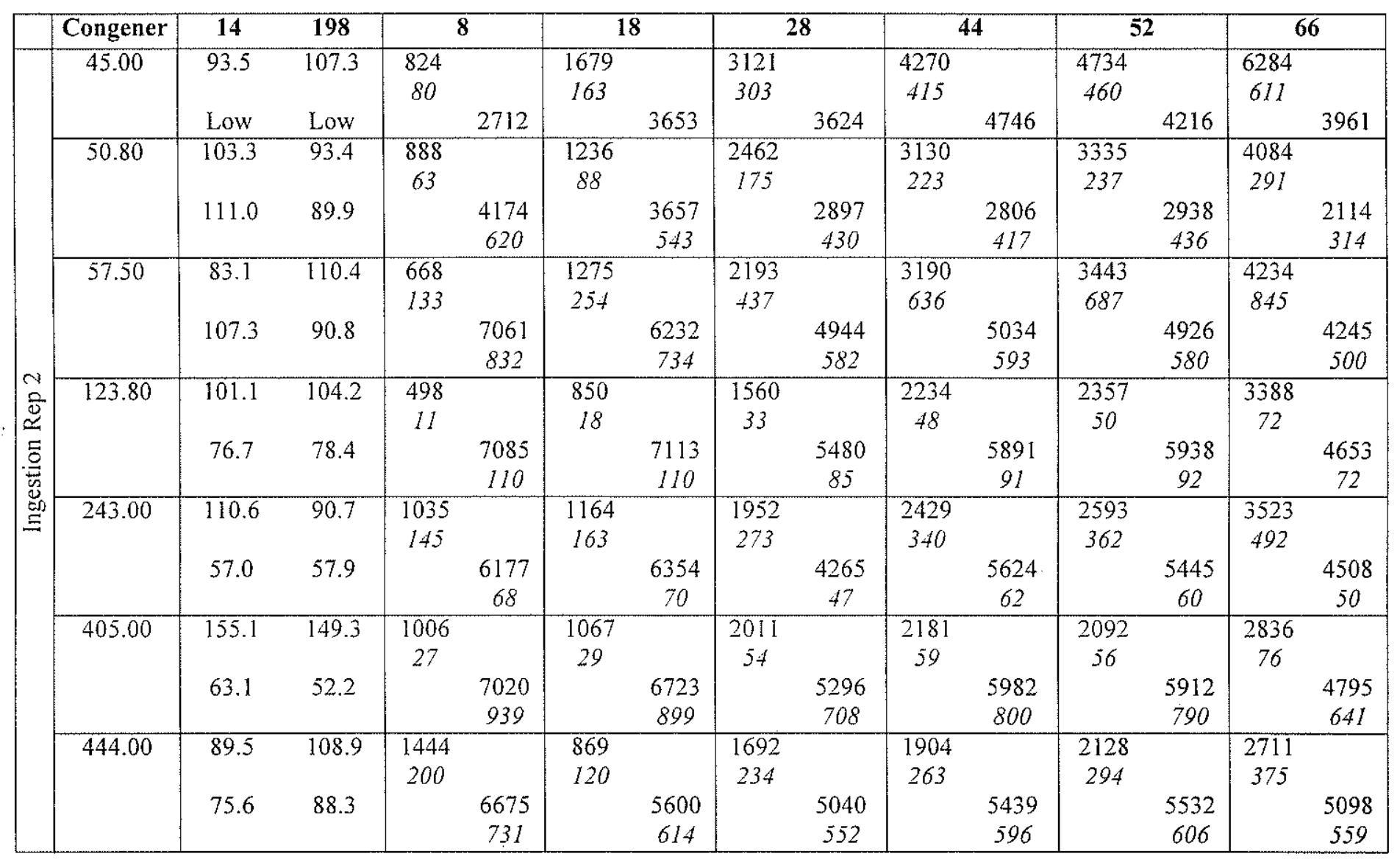




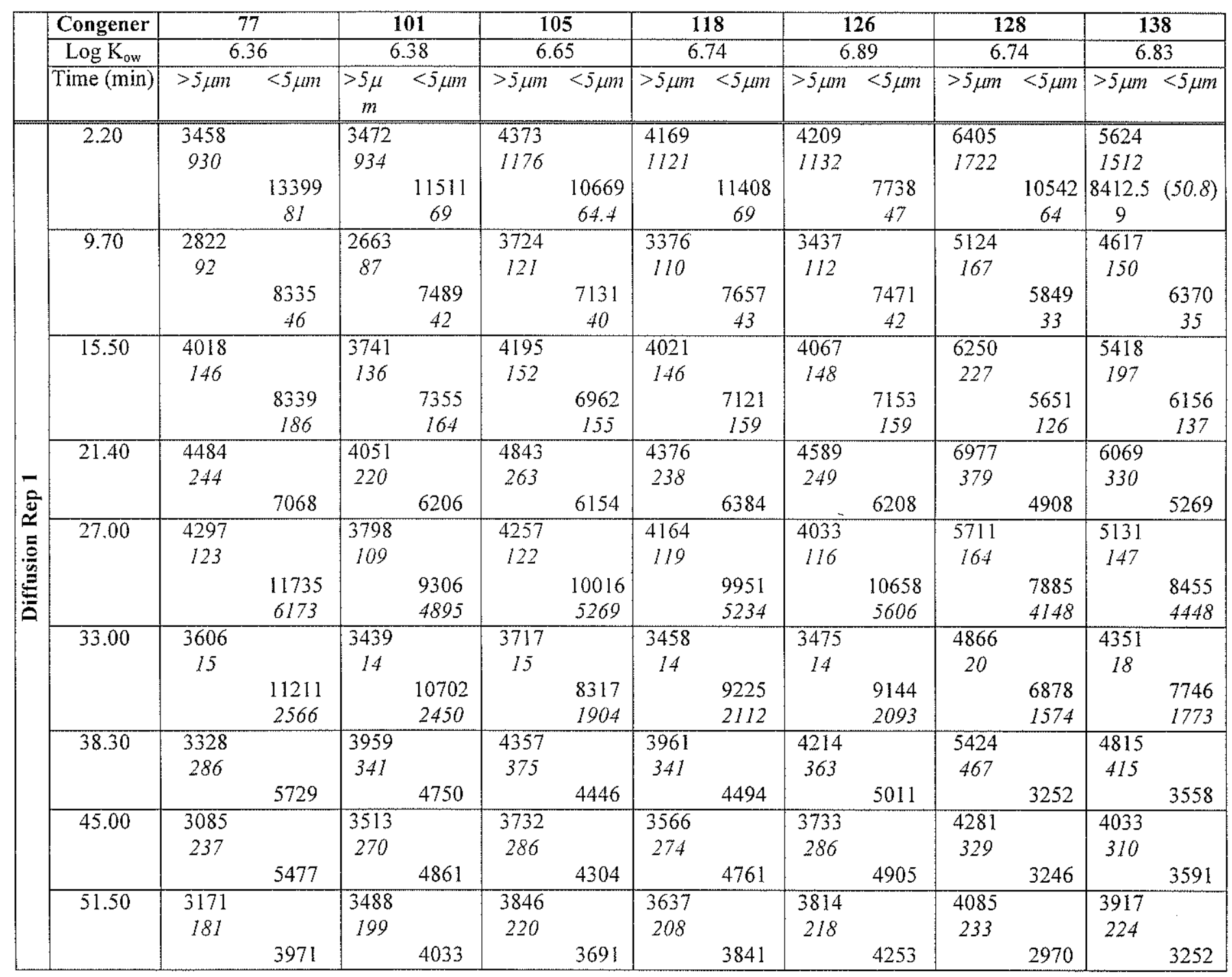




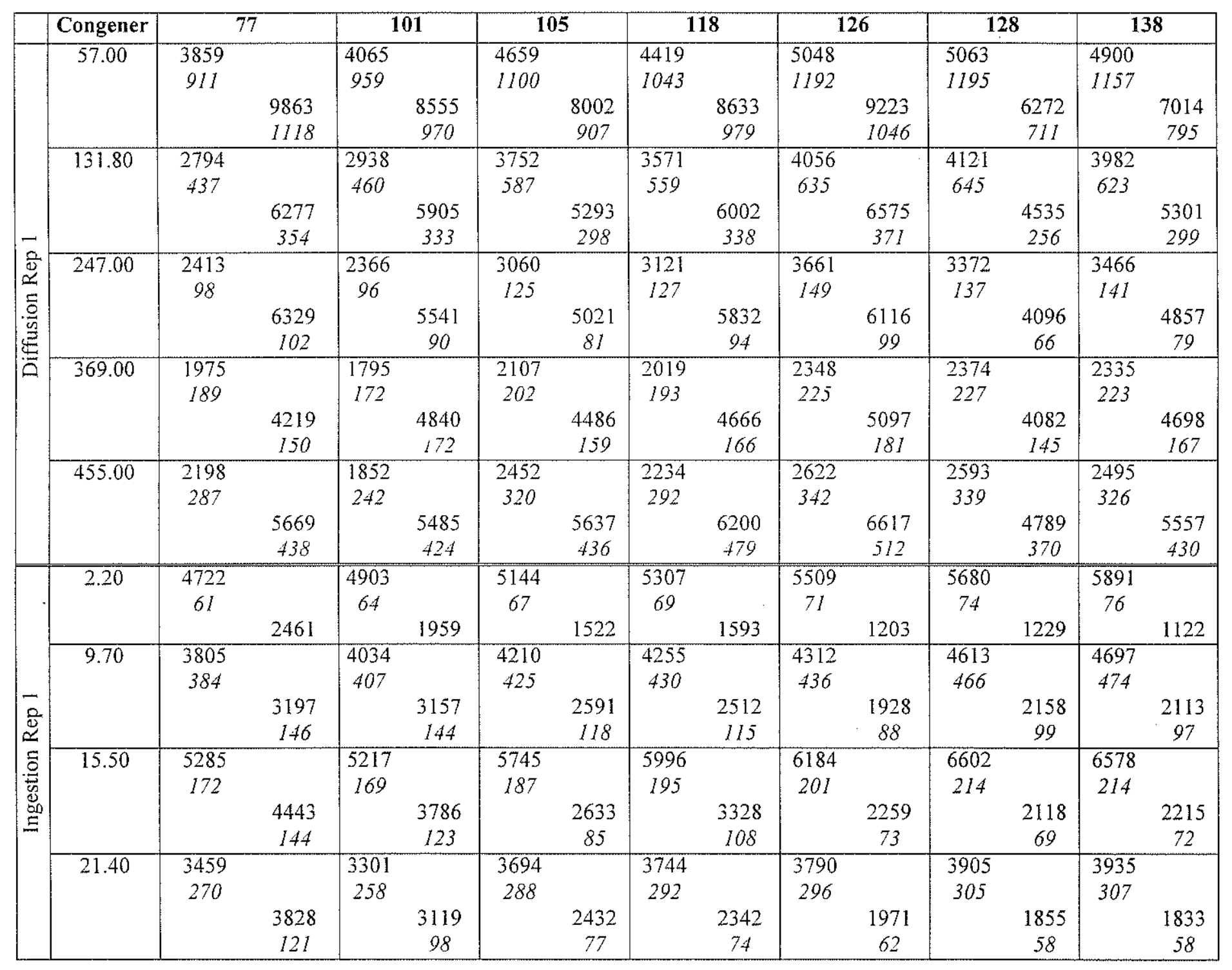




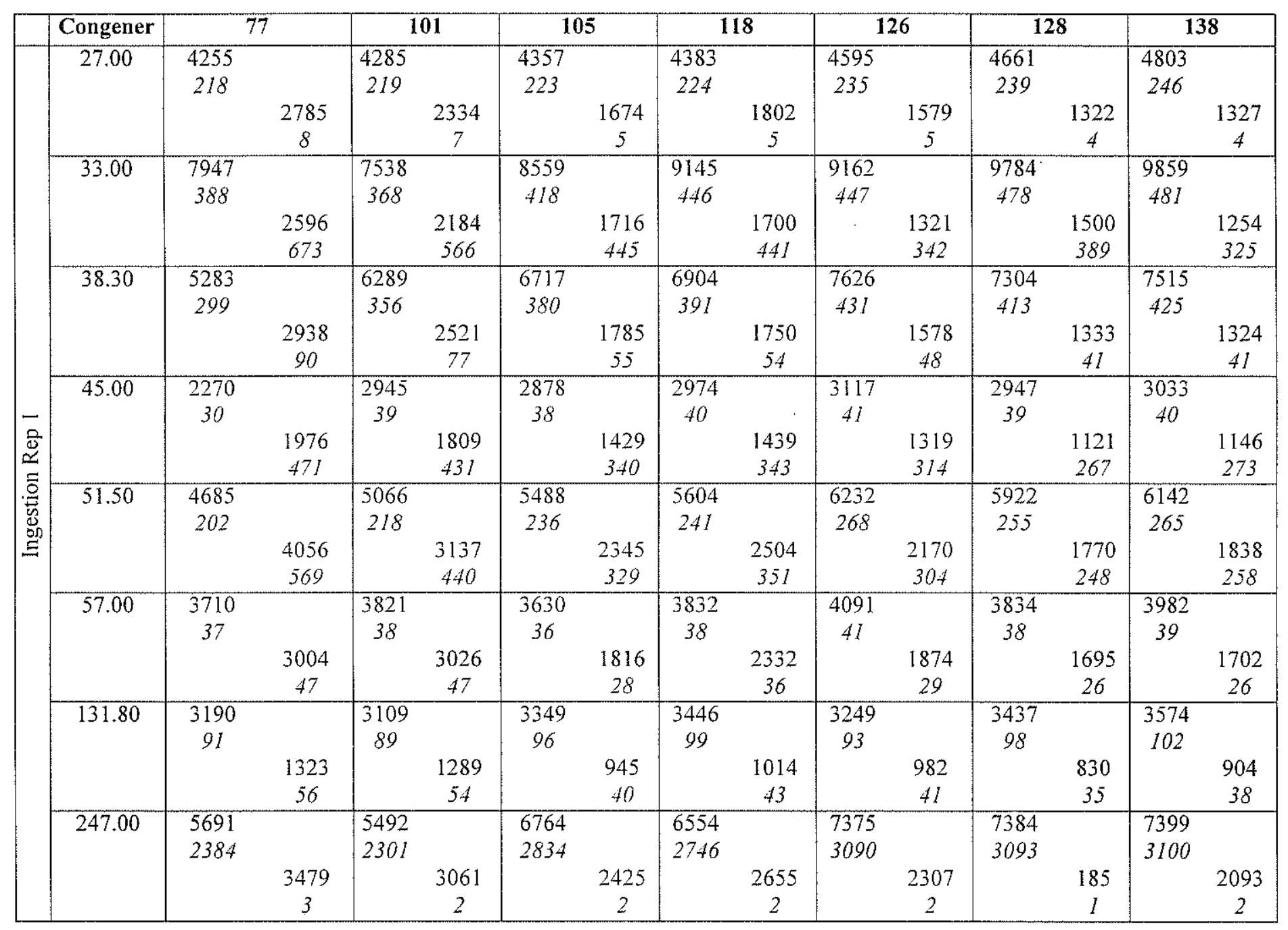




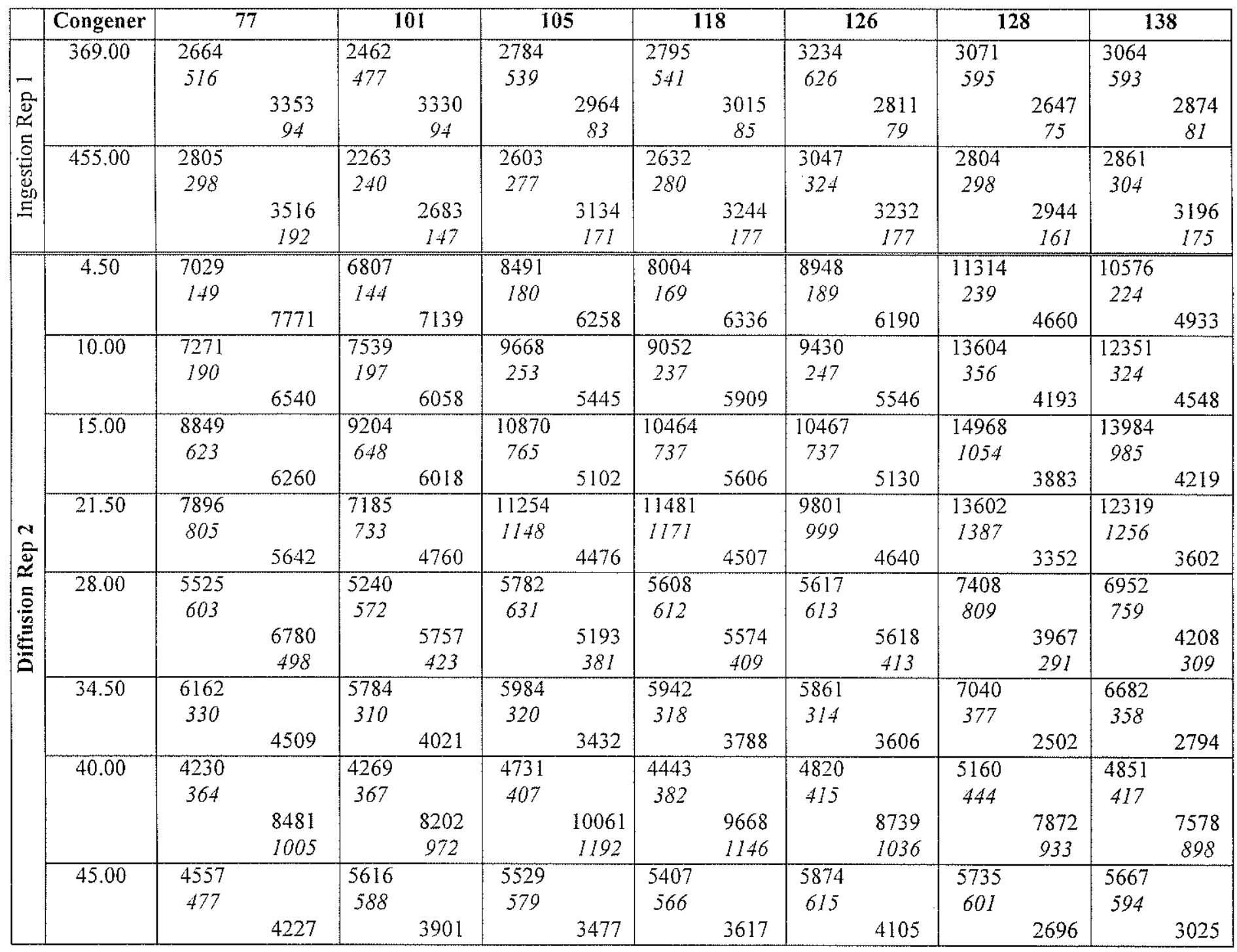




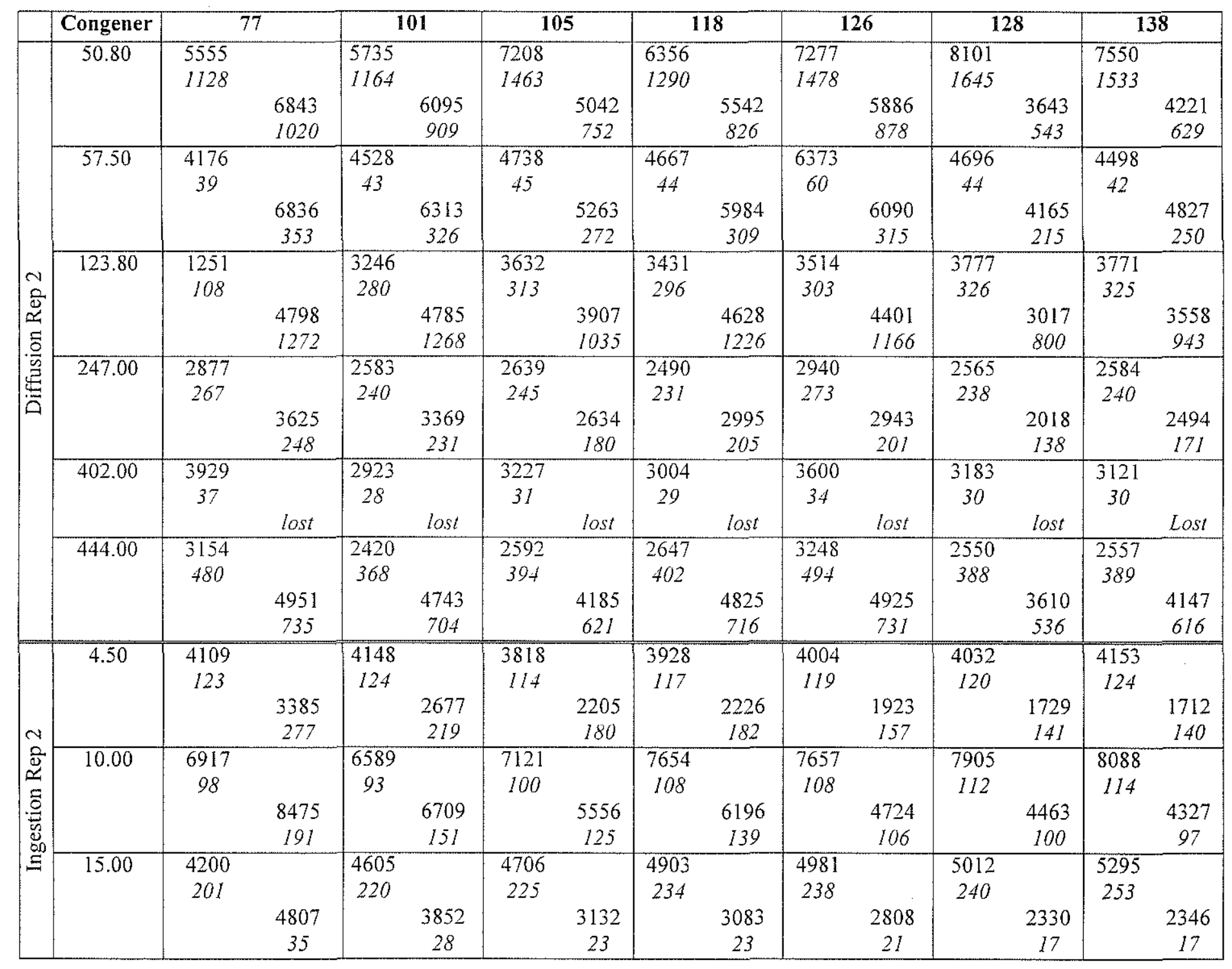




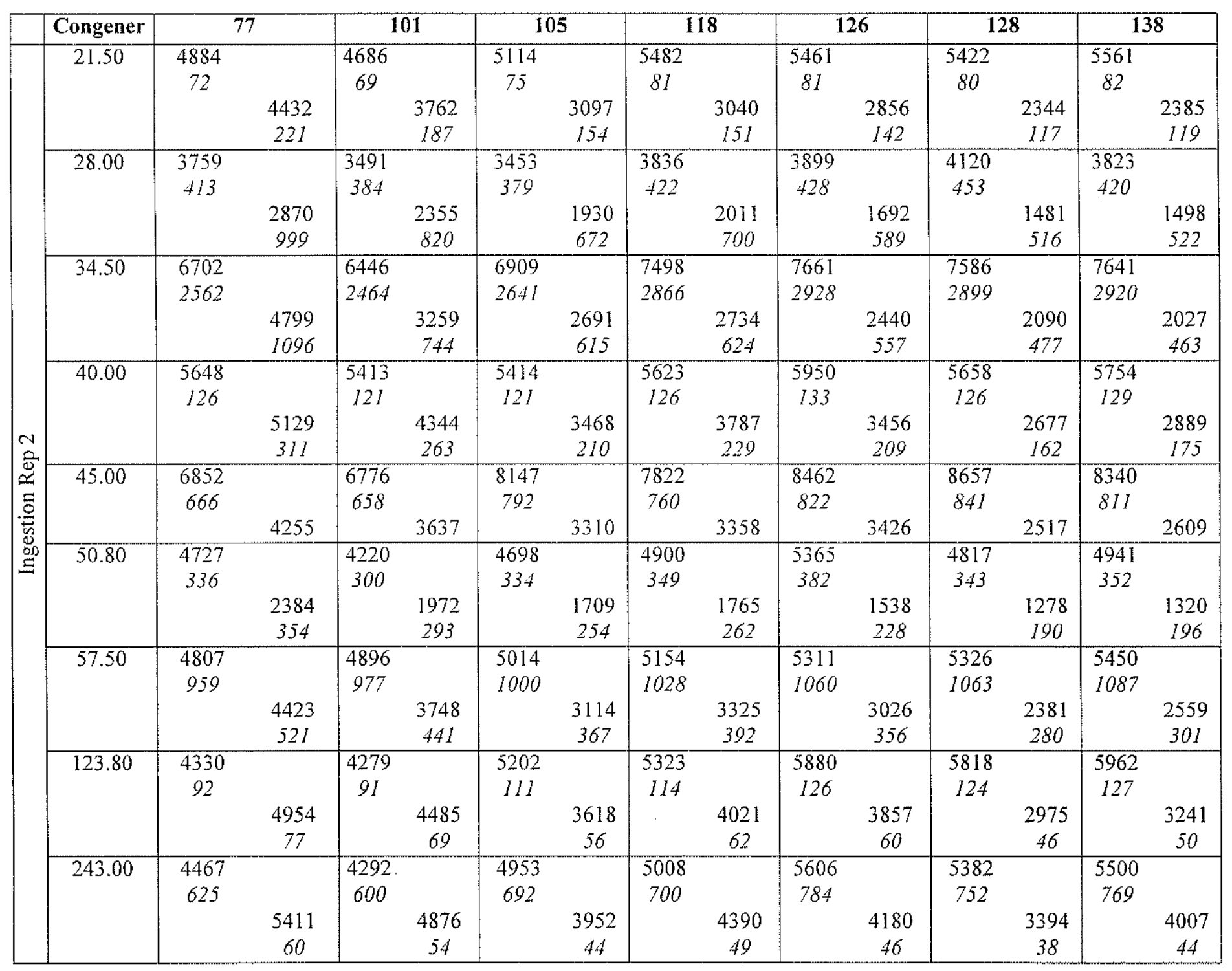




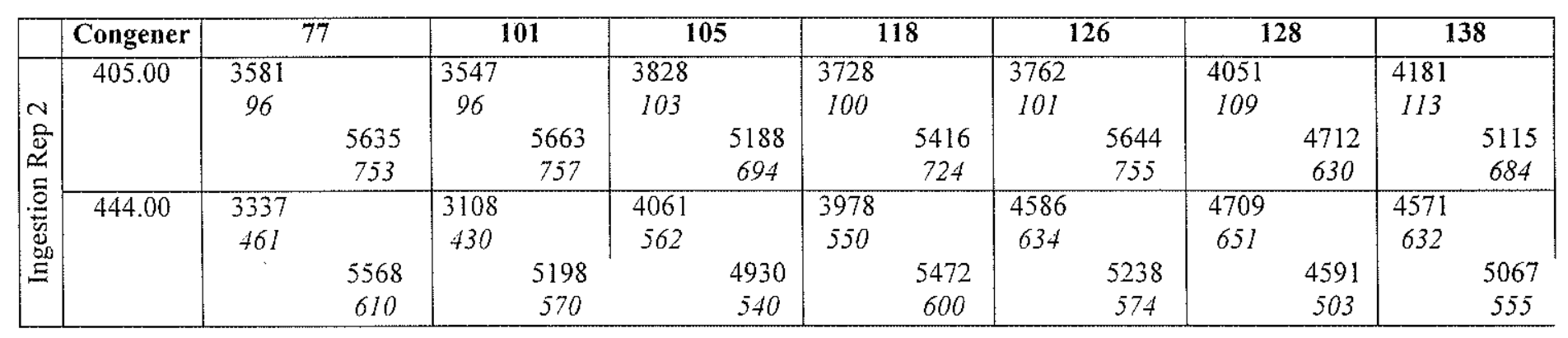

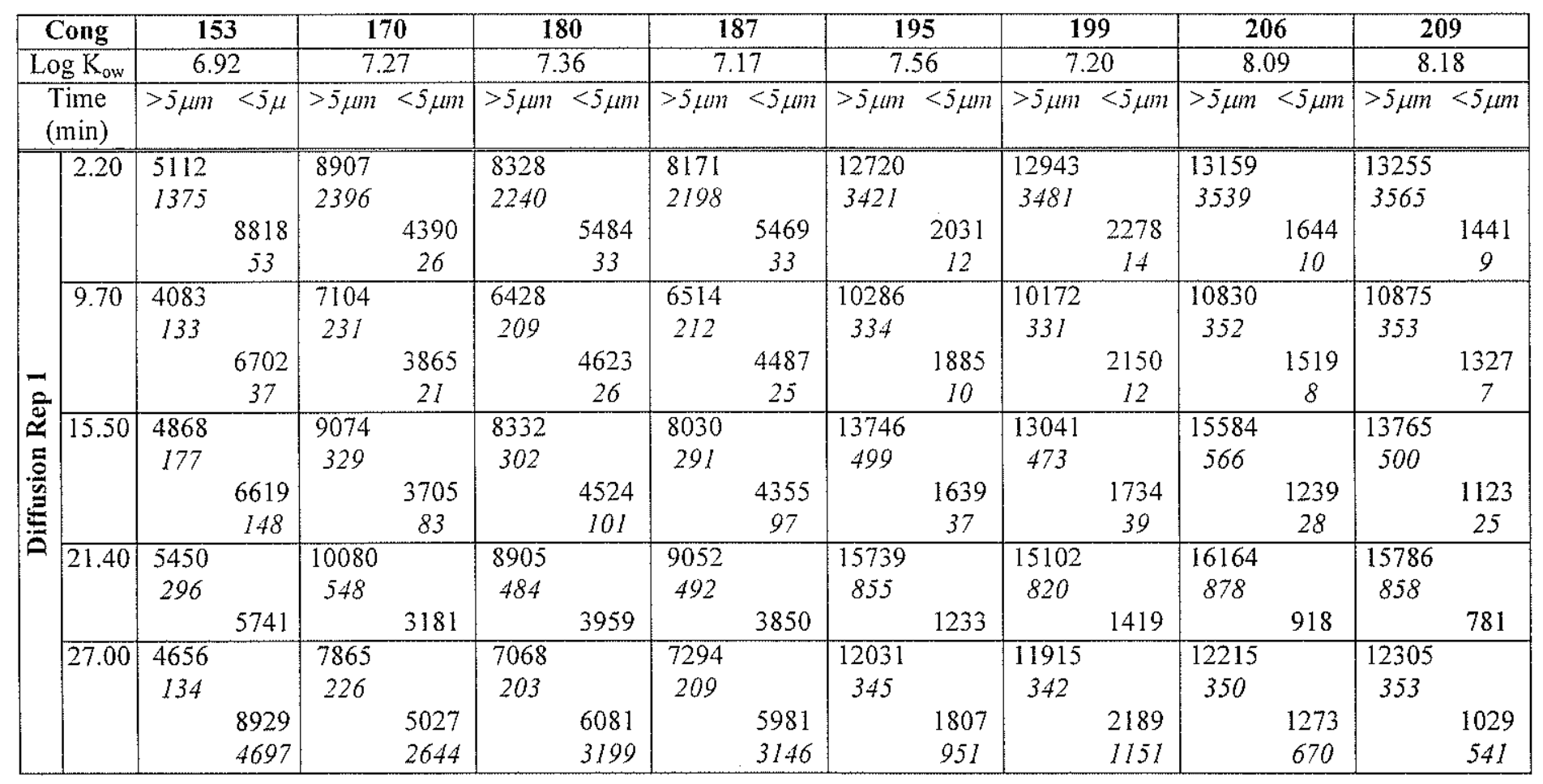




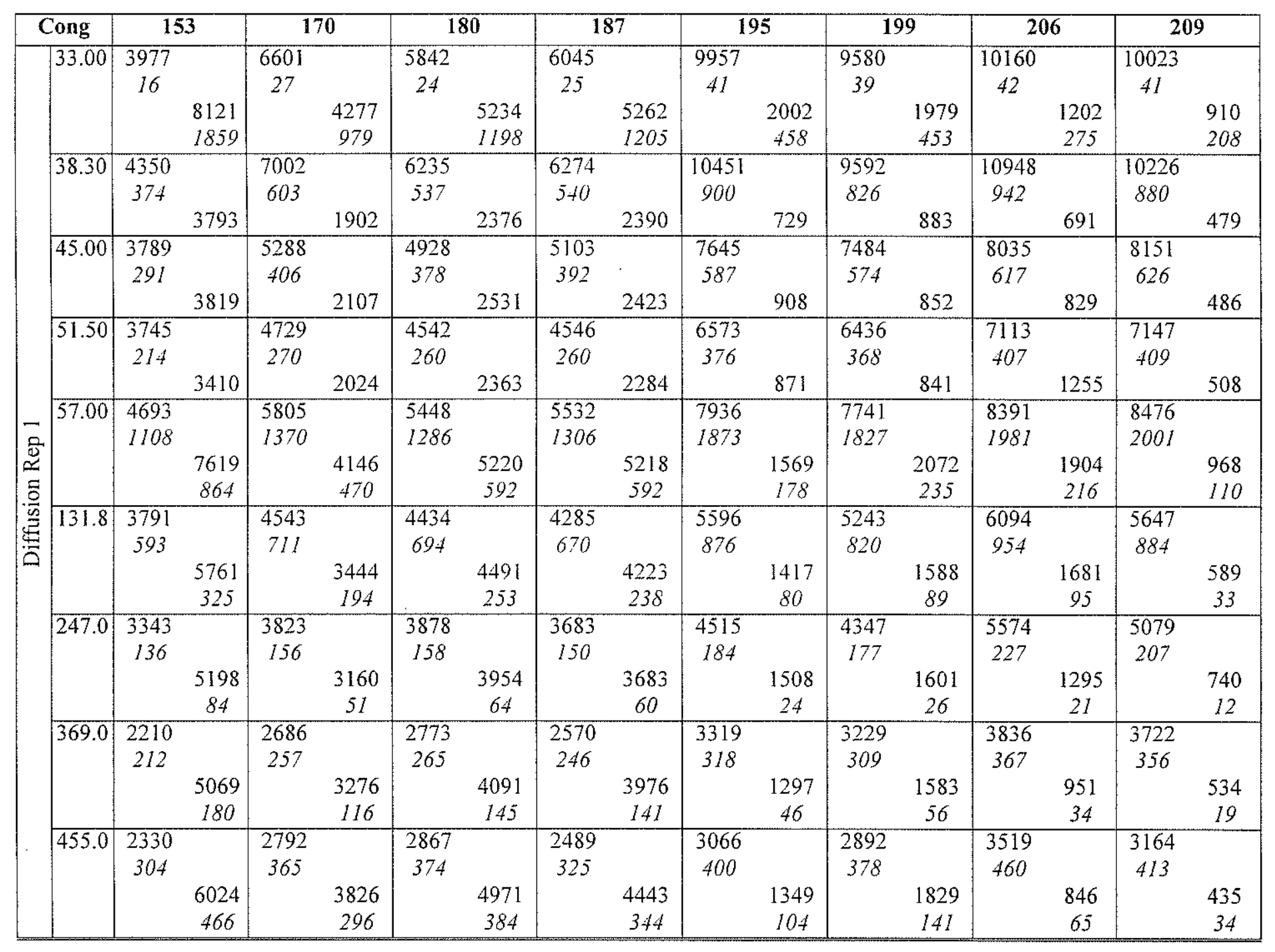




\begin{tabular}{|c|c|c|c|c|c|c|c|c|}
\hline & 153 & 170 & 180 & 187 & 195 & 199 & 206 & 209 \\
\hline 2.20 & $\begin{array}{cc}6133 & \\
80 & \\
& 1068 \\
& 46 \\
\end{array}$ & $\begin{array}{cc}6319 & \\
82 & \\
& 1020.0 \\
& 2 \\
\end{array}$ & $\begin{array}{cc}6724 & \\
87 & \\
& 1046.2 \\
& 4 \\
\end{array}$ & $\begin{array}{cc}6433 & \\
84 & \\
& 828.96\end{array}$ & \begin{tabular}{cc|}
6638 & \\
86 & \\
& 991.08
\end{tabular} & $\begin{array}{c}6957 \\
90\end{array}$ & $\begin{array}{c}7050 \\
92\end{array}$ & $\begin{array}{c}7503 \\
97\end{array}$ \\
\hline 9.70 & 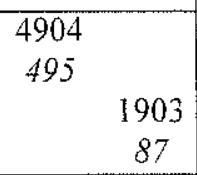 & $\begin{array}{c}5158 \\
521\end{array}$ & $\begin{array}{l}5601 \\
566\end{array}$ & $\begin{array}{c}1615 \\
74 \\
\end{array}$ & $\begin{array}{c}1832 \\
84 \\
\end{array}$ & $\begin{array}{c}1439 \\
66 \\
\end{array}$ & $\begin{array}{c}1599 \\
73\end{array}$ & $\begin{array}{c}6756 \\
682\end{array}$ \\
\hline 15.50 & $\begin{array}{cc}6585 & \\
214 & \\
& 2342 \\
& 76 \\
\end{array}$ & $\begin{array}{c}7083 \\
230\end{array}$ & $\begin{array}{c}1948 \\
63 \\
\end{array}$ & $\begin{array}{c}1852 \\
60 \\
\end{array}$ & $\begin{array}{c}7269 \\
236\end{array}$ & $\begin{array}{l}7666 \\
249\end{array}$ & $\begin{array}{c}7500 \\
244\end{array}$ & $\begin{array}{c}8041 \\
261\end{array}$ \\
\hline $\begin{array}{l}21.40 \\
\text { क्षे }\end{array}$ & $\begin{array}{cc}4070 & \\
318 & \\
& 1880 \\
& 59\end{array}$ & $\begin{array}{c}4303 \\
336\end{array}$ & $\begin{array}{c}4515 \\
352\end{array}$ & $\begin{array}{c}4341 \\
339\end{array}$ & $\begin{array}{c}4751 \\
371\end{array}$ & $\begin{array}{l}5062 \\
395\end{array}$ & $\begin{array}{c}5294 \\
413\end{array}$ & $\begin{array}{c}5622 \\
439\end{array}$ \\
\hline 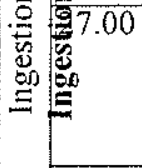 & $\begin{array}{cc}4972 & \\
254 & \\
& 1425 \\
& 4 \\
\end{array}$ & $\begin{array}{l}4946 \\
253\end{array}$ & $\begin{array}{l}5182 \\
265\end{array}$ & $\begin{array}{l}5295 \\
271\end{array}$ & $\begin{array}{c}5229 \\
268\end{array}$ & $\begin{array}{c}5645 \\
289\end{array}$ & $\begin{array}{c}5441 \\
278\end{array}$ & $\begin{array}{c}5990 \\
307\end{array}$ \\
\hline 33.00 & $\begin{array}{c}1001 \\
259\end{array}$ & $\begin{array}{c}10611 \\
518\end{array}$ & $\begin{array}{c}10915 \\
533\end{array}$ & $\begin{array}{c}10704 \\
523\end{array}$ & $\begin{array}{c}10826 \\
528\end{array}$ & $\begin{array}{c}11254 \\
549\end{array}$ & $\begin{array}{c}11919 \\
582\end{array}$ & $\begin{array}{c}11298 \\
551\end{array}$ \\
\hline 38.30 & $\begin{array}{c}7753 \\
439\end{array}$ & $\begin{array}{c}8053 \\
455\end{array}$ & $\begin{array}{c}1063 \\
33 \\
\end{array}$ & $\begin{array}{r}8147 \\
461 \\
\end{array}$ & $\begin{array}{c}8479 \\
480\end{array}$ & $\begin{array}{r}8601 \\
486\end{array}$ & $\begin{array}{c}9634 \\
545\end{array}$ & $\begin{array}{c}9390 \\
531\end{array}$ \\
\hline 45.00 & $\begin{array}{c}3160 \\
42\end{array}$ & $\begin{array}{c}3270 \\
44\end{array}$ & $\begin{array}{l}1073 \\
255\end{array}$ & $\begin{array}{c}3283 \\
44\end{array}$ & $\begin{array}{c}3548 \\
47\end{array}$ & $\begin{array}{c}3570 \\
48\end{array}$ & $\begin{array}{c}3839 \\
51\end{array}$ & $\begin{array}{c}4166 \\
55\end{array}$ \\
\hline
\end{tabular}




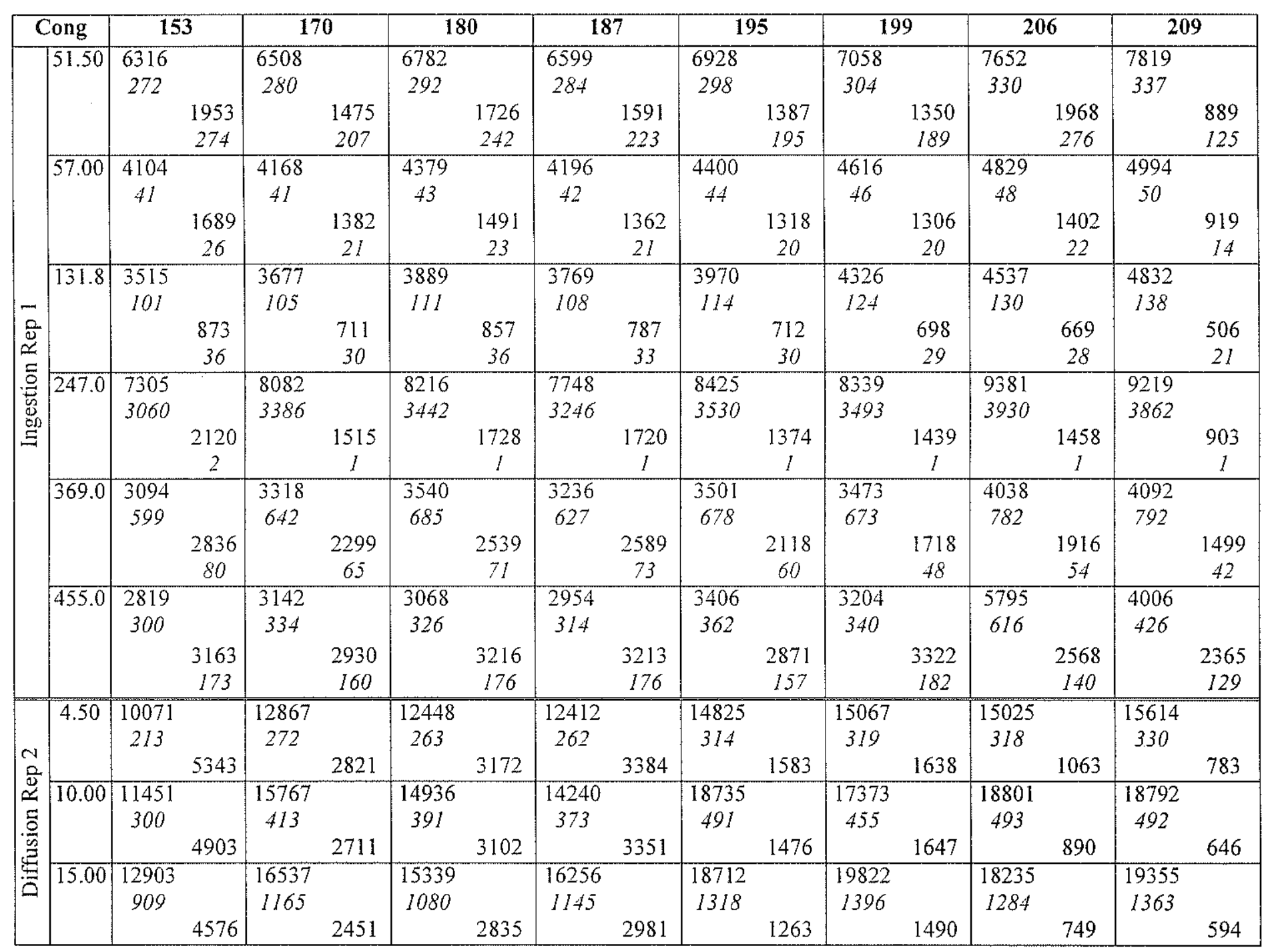




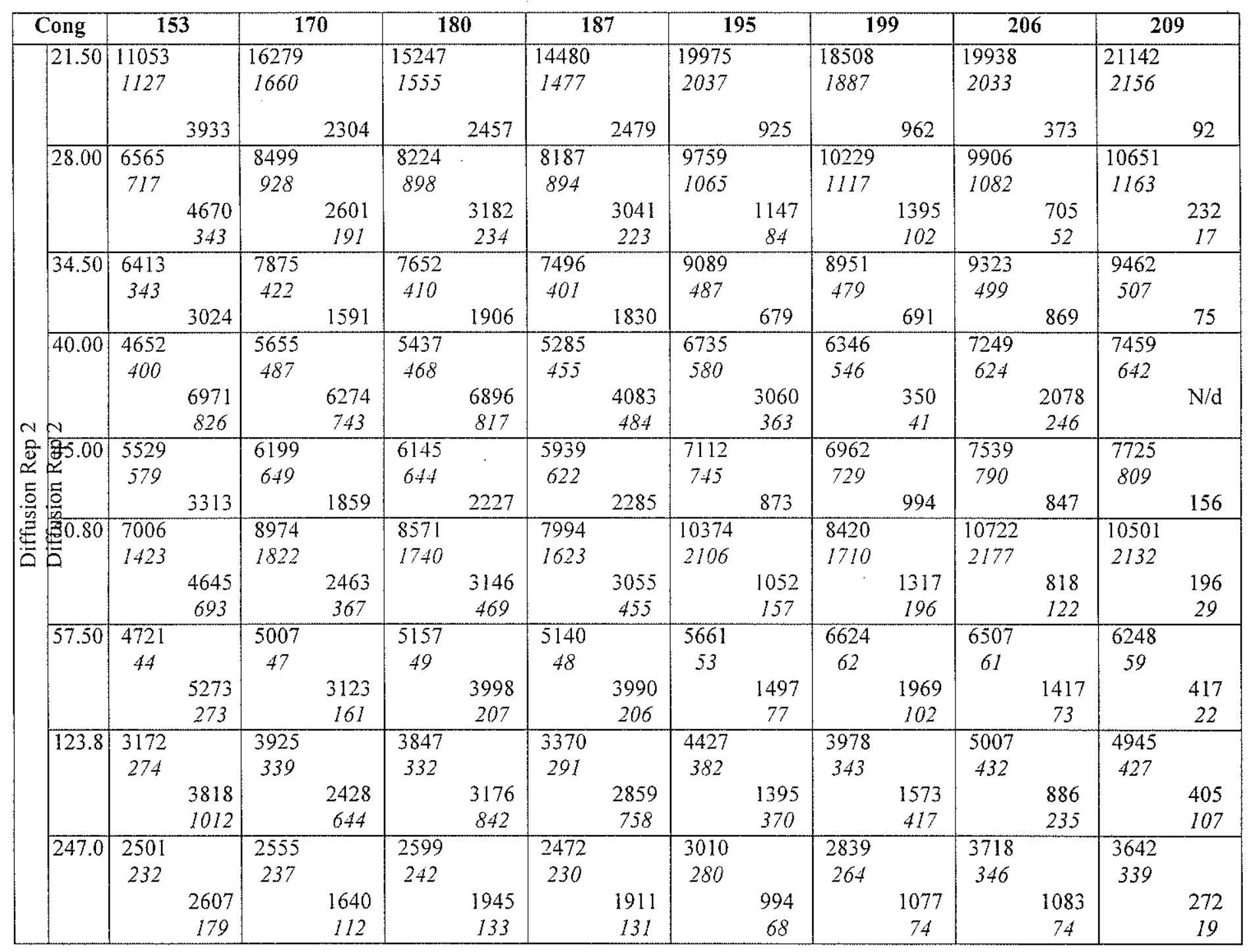




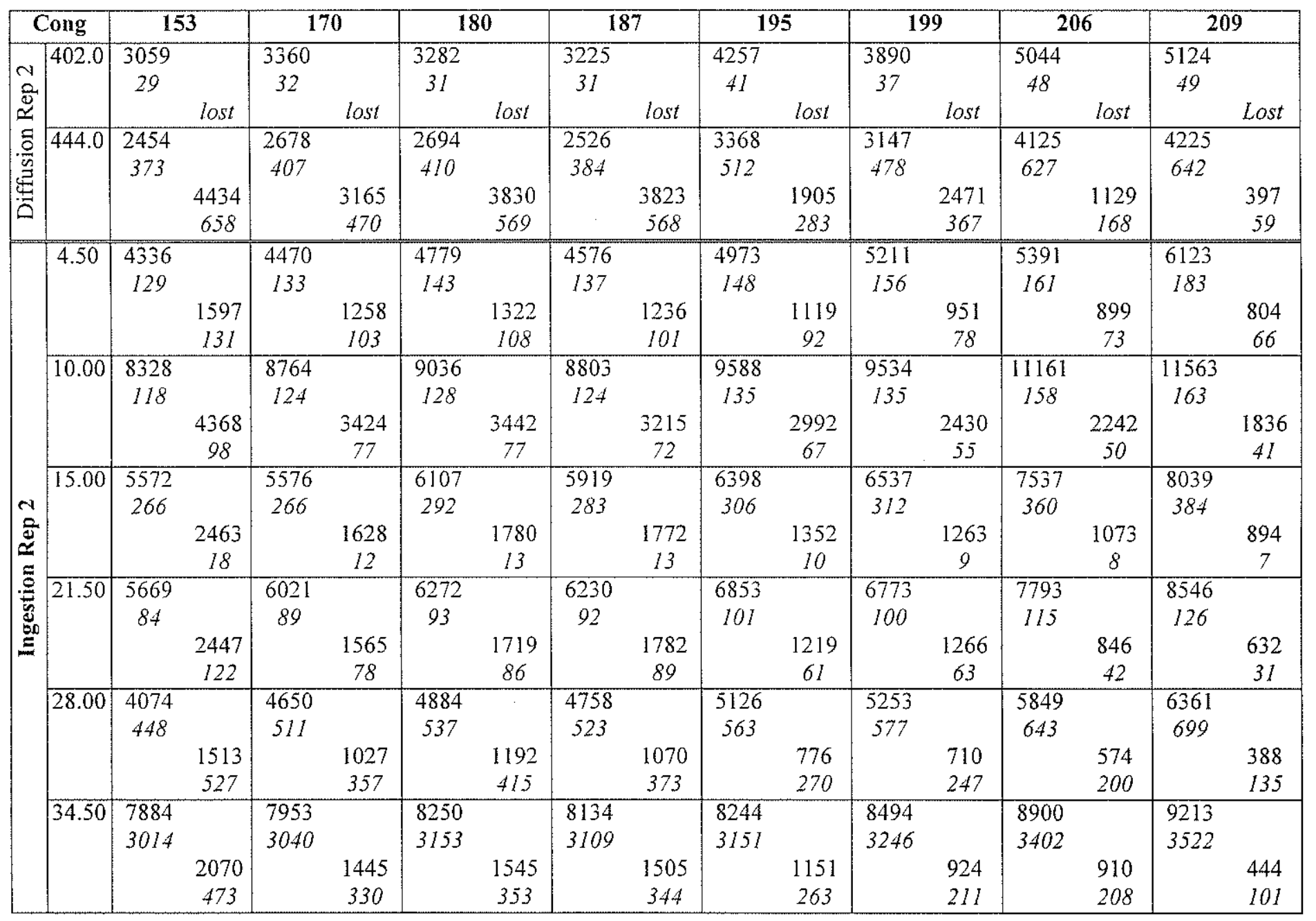




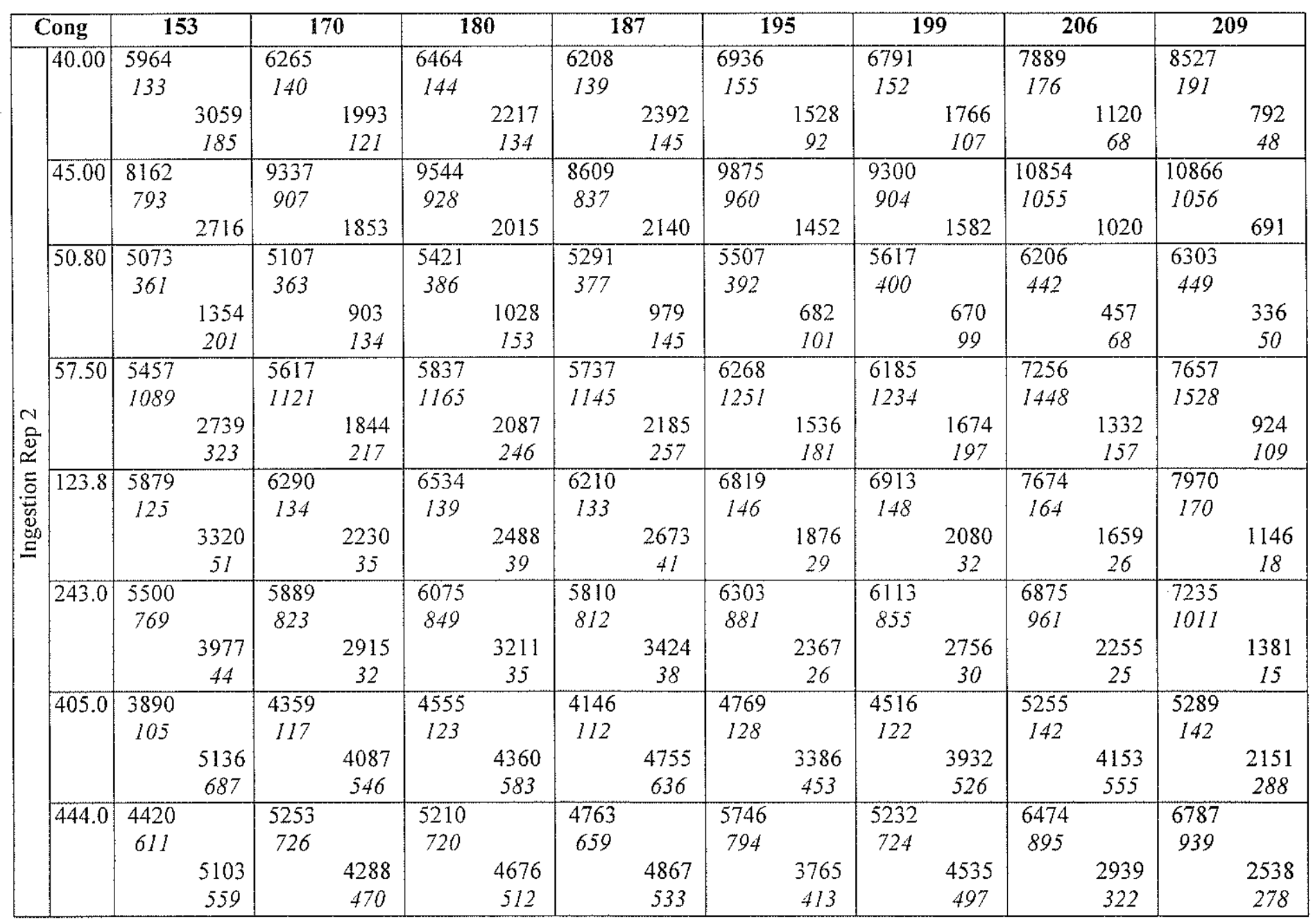




\section{Appendix $C$ - Correction to equation in Cornelissen et al. (1997)}

The equation in Cornelissen et al. (1997) is used to determine the rate constants for desorption from different parts of a solid particle. Tenax resin added to the system removes contaminants within the aqueous phase. The aqueous phase is in equilibrium with both the slow- and rapid-desorbing phases of the solid particle. Interpretation of the Tenax resin results requires the separation of the two desorbing processes. The equation in the publication is:

$$
\text { (1) } \frac{S_{1}}{S_{0}}=F_{c t u} e^{-k_{c x} t}+F_{s t o w} e^{-k_{s t u m} t}+F_{r a p} e^{-k_{r a p} t}
$$

where: $S_{t}$ and $S_{0}$ are the contaminant mass in the solid phase at time $t$ and 0 , respectively; $\mathrm{F}_{\mathrm{ac}}, \mathrm{F}_{\text {slow, }}$ and $\mathrm{F}_{\text {rap }}$ are the contaminant fractions in the aqueous, slow- and rapiddesorbing phases, respectively, $\mathrm{k}_{\mathrm{ex}}$ is the Tenax resin extraction rate constant, $\mathrm{k}_{\text {slow }}$ is the slow-desorbing solid phase extraction rate constant and $\mathrm{k}_{\text {rap }}$ is the rapid-desorbing solid phase extraction rate constant (all in $1 /$ time). This analysis assumes that the extraction of aqueous contaminants by the Tenax resin and the desorption from both the slow- and rapid-desorbing phases are first-order processes and can be described by simple exponential functions. This assumption is valid for the system in question. However, these processes are not additive as suggested by the above equation. Inter-related processes such as desorption from solid particles and subsequent adsorption by Tenax resin must be described by complex exponential functions that incorporate the rate constants within them. The integrated solution is derived from the differential rate equations describing the transport of contaminant among the different phases.

Unlike the system examined by Cornelissen et al. (1997), this system has only one compartment in equilibrium with the aqueous phase. The authors do not consider equilibrium between the de-sorbing particle and the aqueous phase because they assume the Tenax resin removes contaminants faster than the back-reaction to the solid phase. In this general derivation, the back-reaction from the aqueous phase to the solid particle is included. The chemical equations used to describe this system are: 


$$
C_{0} \stackrel{k_{1}}{\longrightarrow} C_{1} \stackrel{k_{2}}{\longrightarrow} C_{2} \text { and } C_{0} \stackrel{B}{\longleftarrow} C_{1}
$$

where $C_{n}$ is the contaminant concentration in phases $0,1,2$. The differential rate equations are:

(2) $\frac{d C_{0}}{d t}=-k_{1} C_{0}+B C_{1} \Rightarrow \frac{d C_{0}}{d t}+k_{1} C_{0}-B C_{1}=0$

(3) $\frac{d C_{1}}{d t}=k_{1} C_{0}-B C_{1}-k_{2} C_{1} \Rightarrow \frac{d C_{1}}{d t}-k_{1} C_{0}+B C_{1}+k_{2} C_{1}=0$

(4) $\frac{d C_{2}}{d t}=k_{2} C_{1} \Rightarrow C_{1}=\frac{d C_{2} / d t}{k_{2}}$

Using the following derivation, the integrated solution to this system of equations can be ascertained.

Equation (3) is substituted into Equation (1).

(5) $\frac{d C_{0}}{d t}+k_{1} C_{0}-B\left(\frac{d C_{2} / d t}{k_{2}}\right)=0$

This rearranges to:

(6) $\frac{d C_{2}}{d t}=\frac{k_{2}}{B}\left(\frac{d C_{0}}{d t}+k_{1} C_{0}\right)$

Taking the derivative with respect to time yields:

(7) $\frac{d^{2} C_{2}}{d t^{2}}=\frac{k_{2}}{B}\left(\frac{d^{2} C_{0}}{d t^{2}}+k_{1} \frac{d C_{0}}{d t}\right)$

Equation (4) is substituted into Equation (3).

(8) $\frac{d}{d t}\left(\frac{d C_{2} / d t}{k_{2}}\right)-k_{1} C_{0}+\left(B+k_{2}\right)\left(\frac{d C_{2} / d t}{k_{2}}\right)=0$

This rearranges to:

(9) $\frac{1}{k_{2}} \frac{d^{2} C_{2}}{d t^{2}}-k_{1} C_{0}+\frac{\left(B+k_{2}\right)}{k_{2}} \frac{d C_{2}}{d t}=0$

Substitute (7) and (6) in (9). 


$$
\frac{1}{k_{2}}\left[\frac{k_{2}}{B}\left(\frac{d^{2} C_{0}}{d t^{2}}+k_{1} \frac{d C_{0}}{d t}\right)\right]-k_{1} C_{0}+\frac{B+k_{2}}{k_{2}}\left[\frac{k_{2}}{B}\left(\frac{d C_{0}}{d t}+k_{1} C_{0}\right)\right]=0
$$

Multiply through to give:

$$
\frac{1}{B} \frac{d^{2} C_{0}}{d t^{2}}+\frac{k_{1}}{B} \frac{d C_{0}}{d t}-k_{1} C_{0}+\frac{B+k_{2}}{B}\left(\frac{d C_{0}}{d t}+k_{1} C_{0}\right)=0
$$

Multiply entire equation by $B$ :

$$
\frac{d^{2} C_{0}}{d t^{2}}+k_{1} \frac{d C_{0}}{d t}-k_{1} B C_{0}+\left(B+k_{2}\right)\left(\frac{d C_{0}}{d t}+k_{1} C_{0}\right)=0
$$

Expand and combine all $C_{0}$ terms:

$$
\frac{d^{2} C_{0}}{d t^{2}}+\frac{d C_{0}}{d t}\left(k_{1}+k_{2}+B\right)+C_{0}\left(k_{1} k_{2}\right)=0
$$

Let

$C_{0}=\alpha e^{-\lambda t}$ where $\alpha, \lambda$ are constants

Substitute $\mathrm{C}_{0}$ and its time derivatives into equation (13).

$$
\lambda^{2} \alpha e^{-\lambda t}-\lambda \alpha e^{-\lambda t}\left(k_{1}+k_{2}+B\right)+\alpha e^{-\lambda t}\left(k_{1} k_{2}\right)=0
$$

Divide by $\alpha \mathrm{e}^{-\lambda t}$ (because $\alpha$ is not zero):

$$
\lambda^{2}-\lambda\left(k_{1}+k_{2}+B\right)+k_{1} k_{2}=0
$$

The roots of this equation, $\lambda_{1}$ and $\lambda_{2}$ are determined using the quadratic formula:

$$
\begin{aligned}
& \lambda_{1}=\frac{\left(k_{1}+k_{2}+B\right)+\sqrt{\left(k_{1}+k_{2}+B\right)^{2}-4 k_{1} k_{2}}}{2} \\
& \lambda_{2}=\frac{\left(k_{1}+k_{2}+B\right)-\sqrt{\left(k_{1}+k_{2}+B\right)^{2}-4 k_{1} k_{2}}}{2}
\end{aligned}
$$

Therefore:

$$
C_{0}=\alpha_{1} e^{-\lambda_{1}{ }^{\prime}}+\alpha_{2} e^{-\lambda_{2} \prime}
$$

where $\alpha_{\mathrm{n}}, \lambda_{\mathrm{n}}$ are constant.

Substitute $\mathrm{C}_{0}$ into equation (16) and rearrange:

$$
C_{1}=\frac{1}{B}\left(\frac{d C_{0}}{d t}+k_{1} C_{0}\right)
$$




$$
C_{1}=\frac{1}{B}\left[\alpha_{1}\left(-\lambda_{1}\right) e^{-\lambda_{1}^{\prime}}+\alpha_{2}\left(-\lambda_{2}\right) e^{-\lambda_{2} t}+k_{1}\left(\alpha_{1} e^{-\lambda_{1} t}+\alpha_{2} e^{-\lambda_{2} \prime}\right)\right.
$$

This rearranges to:

$$
C_{1}=\frac{\alpha_{1}}{B}\left(k_{1}-\lambda_{1}\right) e^{-\lambda_{1} \prime}+\frac{\alpha_{2}}{B}\left(k_{1}-\lambda_{2}\right) e^{-\lambda_{2}{ }^{\prime}}
$$

The coefficients $\alpha_{n}$ can be defined in terms of the rate constants using the initial conditions. The initial conditions for this system are: $\mathrm{C}_{0}(0)=\mathrm{F}_{0} *$ Tot and $\mathrm{C}_{1}(0)=\mathrm{F}_{1}$ *Tot. This yields

$$
C_{0}^{(0)}=F_{0} T o t=\alpha_{1}+\alpha_{2} \Rightarrow \alpha_{1}=F_{0} \text { Tot }-\alpha_{2}
$$

and

$$
C_{1}^{(0)}=F_{1} T o t=\frac{\alpha_{1}}{B}\left(k_{1}-\lambda_{1}\right)+\frac{\alpha_{2}}{B}\left(k_{1}-\lambda_{2}\right)
$$

These two equations are combined to give:

$$
F_{1} \operatorname{Tot}=\frac{F_{0} \operatorname{Tot}-\alpha_{2}}{B}\left(k_{1}-\lambda_{1}\right)+\frac{\alpha_{2}}{B}\left(k_{1}-\lambda_{2}\right)
$$

This equation is rearranged to yield the solution for $\alpha_{2}$ :

$\alpha_{2}=\frac{\operatorname{Tot}\left(F_{1} B-F_{0} k_{1}+F_{0} \lambda_{1}\right)}{\lambda_{1}-\lambda_{2}}$

The other coefficient, $\alpha_{1}$, is determined by substitution of the above into equation (19).

$$
\alpha_{1}=\frac{\operatorname{Tot}\left(F_{0} k_{1}-F_{0} \lambda_{2}-F_{1} B\right)}{\lambda_{1}-\lambda_{2}}
$$




\section{Citations}

Abramowicz D. A. (1990) Aerobic and anaerobic biodegradation of PCBs: A review. Critical Reviews in Biotechnology 10, 241-251.

Adey W. H. and Loveland K. (1991) Dynamic Aquaria. Academic Press, Inc.

Alberts B., Bray D., Lewis J., Raff M., Roberts K., and Watson J. D. (1983) Molecular Biology of the Cell. Garland Publishing, Inc.

Aluwihare L. I. and Repeta D. J. (1999) A comparison of the chemical characteristics of oceanic DOM and extracellular DOM produced by marine algae. Marine Ecology Progress Series 186, 105-117.

Aluwihare L. I., Repeta D. J., and Chen R. F. (1997) A major biopolymeric component to dissolved organic carbon in surface sea water. Nature 387, 166-169.

Andersen O. K., Goldman J. C., Caron D. A., and Dennett M. R. (1986) Nutrient cycling in a microflagellate food chain III. Phosphorus dynamics. Marine Ecology Progress Series 31, 47-55.

Andersson A., Selstam E., and Hagstrom A. (1993) Vertical transport of lipid in seawater. Marine Ecology Progress Series 98, 149-155.

Aveyard R. and Mitchell R. W. (1969) Distribution of n-alkanols between water and nalkanes. Transactions of the Faraday Society 65, 2645-2653.

Axelman J., Broman D., and Naf C. (1997) Field measurements of PCB partitioning between water and planktonic organisms: Influence of growth, particle size, and solute-solvent interactions. Environmental Science and Technology 31, 665-669.

Azam F., Fenchel T., Field J. G., Gray J. S., Meyer-Reil L. A., and Thingstad F. (1983) The ecological role of water-column microbes in the sea. Marine Ecology Progress Series 10, 257-263.

Backhus D. A. and Gschwend P. M. (1990) Fluorescent polycyclic aromatic hydrocarbons as probes for studying the impact of colloids on pollutant transport in groundwater. $E S \& T 24,1214-1223$.

Baker J. E., Capel P. D., and Eisenreich S. J. (1986) Influence of colloids on sedimentwater partition coefficients of polychlorobiphenyl congeners in natural waters. ES\&T 20, 1136-1143.

Baker J. E. and Eisenreich S. J. (1989) PCBs and PAHs as tracers of particulate dynamics in large lakes. Journal of Great Lakes Research 15(1), 84-103.

Baker J. E., Eisenreich S. J., and Eadie B. J. (1991) Sediment trap fluxes and benthic recycling of organic carbon, polycyclic aromatic hydrocarbons, and polychlorobiphenyl congeners in Lake Superior. Environmental Science and Technology 25, 500-509. 
Barbeau K. (1998) The influence of protozoan grazing on the marine geochemistry of particle reactive trace metals. $\mathrm{PhD}$, MIT/WHOI.

Barbeau K., Kujawinski E. B., and Moffett J. W. (submitted) Remineralization and recycling of iron, thorium and organic carbon by heterotrophic marine protozoa. .

Barbeau K. and Moffett J. W. (submitted) Laboratory and field studies of colloidal iron oxide dissolution as mediated by phagotrophy and photolysis. .

Barbeau K., Moffett J. W., Caron D. A., Croot P. L., and Erdner D. L. (1996) Role of protozoan grazing in relieving iron limitation of phytoplankton. Nature 380, 6164.

Barbeau K. A. and Moffett J. W. (1998) Dissolution of iron oxides by phagotrophic protists: Using a novel method to quantify reaction rates. Environmental Science and Technology 32, 2969-2975.

Bedard D. L. and May R. J. (1996) Characterization of the polychlorinated biphenyls in the sediments of Woods Pond: Evidence for microbial dechlorination of Aroclor 1260 in situ. Environmental Science and Technology 30, 237-245.

Benner R., Pakulski J. D., McCarthy M., Hedges J. I., and Hatcher P. G. (1992) Bulk chemical characteristics of dissolved matter in the ocean. Science 255, 1561-1564.

Bergen B. J., Nelson W. G., and Pruell R. J. (1993) Partitioning of polychlorinated biphenyl congeners in the seawater of New Bedford Harbor, Massachusetts. Environmental Science and Technology 27, 938-942.

Bergen B. J., Nelson W. G., and Pruell R. J. (1996) Comparison of nonplanar and coplanar PCB congener partitioning in seawater and bioaccumulation in blue mussels (Mytilus edulis). Environmental Toxicology and Chemistry 15, 15171523.

Bergen B. J., Quinn J. G., and Parrish C. C. (1999) Quality assurance study of marine lipid class determination using Chromarod / Iatroscan TLC-FID. submitted.

Bligh E. G. and Dyer W. J. (1959) A rapid method of total lipid extraction and purification. Canadian Journal of Biochemistry and Physiology 37, 911-917.

Bortiatynski J. M., Hatcher P. G., and Minard R. O. (1997) The development of 13C labeling and 13C NMR spectroscopy technique to study the interactions of pollutants with humic substances. In Nuclear Magnetic Resonance Spectroscopy in Environmental Science and Technology (ed. M. A. Nanny, R. A. Minear, and J. A. Leenheer), pp. 26-50. Oxford University Press.

Brahm J. (1983) Permeability of human red cells to a homologous series of aliphatic alcohols. Journal of General Physiology 81, 283-304.

Brownawell B. J. (1986) The role of colloidal organic matter in the marine geochemistry of PCBs. Ph.D., MIT/WHOI. 
Brownawell B. J. (1997) Common headspace partitioning as a method for probing macromolecule hydrophobicity. 214th ACS National Meeting, 91-92.

Brownawell B. J. and Farrington J. W. (1986) Biogeochemistry of PCBs in interstitial waters of a coastal marine sediment. Geochimica et Cosmochimica Acta 50, 157 169.

Burreau S., Axelman J., Broman D., and Jakobsson E. (1997) Dietary uptake in pike (Esox lucius) of some polychlorinated biphenyls, polychlorinated naphthalenes and polybrominated diphenyl ethers administered in natural diet. Environmental Toxicology and Chemistry 16, 2508-2513.

Campfens J. and Mackay D. (1997) Fugacity-based model of PCB bioaccumulation in complex aquatic food webs. Environmental Science and Technology 31, 577-583.

Carlson C. A., Ducklow H. W., and Michaels A. F. (1994) Annual flux of dissolved organic carbon from the euphotic zone in the northwestern Sargasso Sea. Nature 371, 405-408.

Caron D. A., Goldman J. C., Andersen O. K., and Dennett M. R. (1985) Nutrient cycling in a microflagellate food chain: II. Population dynamics and carbon cycling. Marine Ecology Progress Series 24, 243-254.

Caron D. A., Goldman J. C., and Dennett M. R. (1988) Experimental demonstration of the roles of bacteria and bacterivorous protozoa in plankton nutrient cycles. Hydrobiologia 159, 27-40.

Caron D. A., Lim E. L., Miceli G., Waterbury J. B., and Valois F. W. (1991) Grazing and utilization of choococcoid cyanobacteria and heterotrophic bacteria by protozoa in laboratory cultures and a coastal plankton community. Marine Ecology Progress Series 76, 205-217.

Carter C. W. and Suffet I. H. (1982) Binding of DDT to dissolved humic materials. ES\&T 16(11), 735-740.

Chin Y.-P., Aiken G. R., and Danielsen K. M. (1997) Binding of pyrene to aquatic and commercial humic substances: The role of molecular weight and aromaticity. Environmental Science and Technology 31, 1630-1635.

Chin Y.-P. and Gschwend P. M. (1992) Partitioning of Polycyclic Aromatic Hydrocarbons to Marine Porewater Organic Colloids. ES\&T 26(8), 1621-1626.

Chiou C. T., Malcolm R. L., Brinton T. I, and Kile D. E. (1986) Water solubility enhancement of some organic pollutants and pesticides by dissolved humic and fulvic acids. Environmental Science and Technology 20, 502-508.

Connell D. W. (1989) Biomagnification by aquatic organisms - A proposal. Chemosphere 19, 1573-1584. 
Connolly J. P. (1991) Application of a food chain model to polychlorinated biphenyl contamination of the lobster and winter flounder food chains in New Bedford Harbor. Environmental Science and Technology 25, 760-770.

Cornelissen G., Noort P. C. M. v., and Govers H. A. J. (1997) Desorption kinetics of chlorobenzenes, polycyclic aromatic hydrocarbons, and polychlorinated biphenyls: Sediment extraction with Tenax and effects of contact time and solute hydrophobicity. Environmental Toxicology and Chemistry 16, 1351-1357.

Cosovic B. and Vojvodic V. (1989) Adsorption behaviour of the hydrophobic fraction of organic matter in natural waters. Marine Chemistry 28, 183-198.

Cosovic B., Zutic V., Vojdovic V., and Plese T. (1985) Determination of surfactant activity and anionic detergents in seawater and sea surface microlayer in the Mediterranean. Marine Chemistry 17, 127-139.

Derieux S., Fillaux J., and Saliot A. (1998) Lipid class and fatty acid distributions in particulate and dissolved fractions in the north Adriatic Sea. Organic Geochemistry 29, 1609-1621.

Druffel E. R. M., Williams P. M., Bauer J. E., and Ertel J. R. (1992) Cycling of dissolved and particulate organic matter in the open ocean. Journal of Geophysical Research 97, 15639-15659.

Dulfer W. J., Govers H. A. J., and Groten J. P. (1998) Kinetics and conductivity parameters of uptake and transport of polychlorinated biphenyls in the $\mathrm{CaCO}-2$ intestinal cell line model. Environmental Toxicology and Chemistry 17, 493-501.

Dulfer W. J., Groten J. P., and Govers H. A. J. (1996) Effect of fatty acids and the aqueous diffusion barrier on the uptake and transport of polychlorinated biphenyls in Caco-2 cells. Journal of Lipid Research 37, 950-961.

Duursma E. K., Nieuwenhuize J., and Liere J. M. v. (1989) Polychlorinated Biphenyl Equilibria in an Estuarine System. The Science of the Total Environment 79, 141155.

Evans M. S., Noguchi G. E., and Rice C. P. (1991) The biomagnification of polychlorinated biphenyls, toxaphene, and DDT compounds in a Lake Michigan offshore food web. Archives of Envrionmental Contamination and Toxicology 20, 87-93.

Farrington J. W. (1991) Biogeochemical processes governing exposure and uptake of organic pollutant compounds in aquatic organisms. Environmental Health Perspectives 90, 75-84.

Farrington J. W. and Westall J. (1986) Organic Chemical Pollutants in the Oceans and Groundwater: A Review of Fundamental Chemical Properties and Biogeochemistry. In The Role of the Oceans as a Waste Disposal Option (ed. G. Kullenberg), pp. 361-425. D. Reidel Publishing Company. 
Fenchel T. (1980) Suspension feeding in ciliated protozoa: Feeding rates and their ecological significance. Microbial Ecology 6, 13-25.

Fenchel T. (1987) Ecology of Protozoa. Science Tech Publishers, Springer-Verlag.

Fok A. K., Lee Y., and Allen R. (1982) The correlation of digestive vacuole pH and size with the digestive cycle in Paramecium caudatum. Journal of Protozoology 29(3), 409-414.

Frew N. M., Goldman J. C., Dennett M. R., and Johnson A. S. (1990) Impact of phytoplankton-generated surfactants on air-sea gas exchange. Journal of Geophysical Research 95, 3337-3352.

Frost B. (1972) Effects of size and concentration of food particles on the feeding behavior of the marine planktonic copepod Calanus pacificus. Limnology and Oceanography 17, 805-815.

Furukawa K. (1986) Modification of PCBs by bacteria and other microorganisms. In PCBs and the Environment, Vol. 2 (ed. J. S. Waid), pp. 89-100. CRC Press, Inc.

Gagosian R. B. and Stuermer D. H. (1977) The cycling of biogenic compounds and their diagenetically transformed products in seawater. Marine Chemistry 5, 605-632.

Garbarini D. R. and Lion L. W. (1986) The influence of the nature of soil organics on the sorption of toluene and trichloroethylene. Environmental Science and Technology 20, 1263-1269.

Gasparovic B. and Cosovic B. (1994) Electrochemical estimation of the dominant type of surface active substances in seawater samples using $o$-nitrophenol as a probe. Marine Chemistry 46, 179-188.

Gauthier T. D., Seitz W. R., and Grant C. L. (1987) Effect of structural and compositional variations of dissolved humic materials on pyrene Koc values. Environmental Science and Technology 21, 243-248.

Goldman J. C., Caron D. A., and Dennett M. R. (1987) Nutrient cycling in a microflagellate food chain: IV. phytoplankton-microflagellate interactions. Marine Ecology Progress Series 38, 75-87.

Goldman J. C., David A C., Andersen O. K., and Dennett M. R. (1985) Nutrient cycling in a microflagellate food chain: I. Nitrogen dynamics. Marine Ecology Progress Series 24, 231-242.

Grimberg S., Nagel J., and Aitken M. D. (1995) Kinetics of phenanthrene dissolution into water in the presence of nonionic surfactants. Environmental Science and Technology 29, 1480-1487.

Gschwend P. M. and Wu S.-c. (1985) On the constancy of sediment-water partition coefficients of hydrophobic organic pollutants. Environmental Science and Technology 19, 90-96. 
Guillard R. R. L. and Ryther J. H. (1962) Studies on marine planktonic diatoms. I. Cyclotella nana Hustedt and Detonula confervacae (Cleve). Grand Canadian Journal of Microbiology 8, 229-239.

Gundersen K. and Wassmann P. (1990) Use of chloroform in sediment traps: caution advised. Marine Ecology Progress Series 64, 187-195.

Gunnarsson J. S. and Rosenberg R. (1996) Eutrophication increases the association of PCB to dissolved organic matter in marine microcosms. Marine Pollution Bulletin 33, $100-111$.

Gustafsson O. (1997) Physico-chemical speciation and ocean fluxes of polycyclic aromatic hydrocarbons. Ph.D., MIT / WHOI.

Gustafsson O. and Gschwend P. M. (1997) Aquatic colloids: Concepts, definitions, and current challenges. Limnology and Oceanography 42, 519-528.

Gustafsson O., Haghseta F., Chan C., MacFarlane J., and Gschwend P. M. (1997) Quantification of the dilute sedimentary soot phase: Implications for PAH speciation and bioavailability. Environmental Science and Technology 31, 203209.

Hamerlink J. L., Landrum P. F., Bergman H. L., and Benson W. H. (1994) Bioavailability: Physical, Chemical, and Biological Interactions. In SETAC Special Publications Series (ed. T. W. LaPoint, B. T. Walton, and C. H. Ward), pp. 239. CRC Press.

Harvey R. W., Kinner N. E., Bunn A., MacDonald D., and Metge D. (1995) Transport behavior of groundwater protozoa and protozoan microspheres in sandy aquifer sediments. Applied and Environmental Microbiology 61, 209-217.

Hawker D. W. and Connell D. W. (1988) Octanol-water partition coefficients of polychlorinated biphenyl congeners. Environmental Science and Technology 22, 382-387.

Heinbokel J. F. (1978) Studies on the functional role of tintinnids in the Southern California Bight. I. Grazing and growth rates in laboratory cultures. Marine Biology 47, 177-189.

Hofelt C. and Shea D. (1997) Accumulation of organochlorine pesticides and PCBs by semipermeable membrane devices and Mytilus edulis in New Bedford Harbor. Environmental Science and Technology 31, 154-159.

Huckins J. N., Manuweera G. K., Petty J. D., Mackay D., and Lebo J. A. (1993) Lipidcontaining semipermeable membrane devices for monitoring organic contaminants in water. Environmental Science and Technology 27, 2489-2496.

Hunchak-Kariouk K., Schweitzer L., and Suffet I. H. (1997) Partitioning of 2,2',4,4'tetrachlorobiphenyl by the dissolved organic matter in oxic and anoxic porewaters. Environmental Science and Technology 31, 639-645. 
Hunter K. A. and Liss P. S. (1981) Polarographic measurement of surface-active material in natural waters. Water Research 15, 203-215.

Iriberri J., Ayo B., Santamaria E., Barcina I., and Egea L. (1995) Influence of bacterial density and water temperature on the grazing activity of two freshwater ciliates. Freshwater Biology 33, 223-231.

Jumars P. A., Penry D. L., Baross J. A., Perry M. J., and Frost B. W. (1989) Closing the microbial loop: dissolved carbon pathway to heterotrophic bacteria from incomplete ingestion, digestion and absorption in animals. Deep-Sea Research 36, 483-495.

Kannan N., Reusch T. B. H., Schulz-Bull D. E., Petrick G., and Duinker J. C. (1995) Chlorobiphenyls: Model compounds for metabolism in food chain organisms and their potential use as ecotoxicological stress indicators by application of the metabolic slope concept. Environmental Science and Technology 29, 1851-1859.

Kannan N., Tanabe S., Tatsukawa R., and Phillips D. J. (1989) Persistency of highly toxic coplanar PCBs in aquatic ecosystems: Uptake and release kinetics of coplanar PCBs in green lipped mussels (Perna viridis Linnaeus). Environmental Pollution 56, 65-76.

Karickhoff S. W., Brown D. S., and Scott T. A. (1979) Sorption of hydrophobic pollutants on natural sediments. Water Research 13, 241-248.

Kattner G., Gercken G., and Hammer K. D. (1983) Development of lipids during a spring plankton bloom in the northern North Sea. II. Dissolved lipids and fatty acids. Marine Chemistry 14, 163-173.

Keil R. G., Tsamakis E., Fuh C. B., Giddings J. C., and Hedges J. I. (1994) Mineralogical and textural controls on the organic composition of coastal marine sediments: Hydrodynamic separation using SPLITT-fractionation. Geochimica et Cosmochimica Acta 58, 879-893.

Kennicutt M. C. and Jeffrey L. M. (1981) Chemical and GC-MS characterization of marine dissolved lipids. Marine Chemistry 10, 364-387.

Kucklick J. R. and Baker J. E. (1998) Organochlorines in Lake Superior's food web. Environmental Science and Technology 32, 1192-1198.

Lebo J. A., Gale R. W., Petty J. D., Tillitt D. E., Huckins J. N., Meadows J. C., Orazio C. E., Echols K. R., Schroeder D. J., and Inmon L. E. (1995) Use of the semipermeable membrane device as an in situ sampler of waterborne bioavailable PCDD and PCDF residues at sub-parts-per-quadrillion concentrations. Environmental Science and Technology 29, 2886-2892.

Lee C., Hedges J. I., Wakeham S. G., and Zhu N. (1992) Effectiveness of various treatments in retarding microbial activity in sediment trap material and their effects on the collection of swimmers. Limnology and Oceanography 37, 117130. 
Lee C. and Wakeham S. G. (1988) Organic matter in seawater: Biogeochemical processes. In Chemical Oceanography, Vol. 9, pp. 1-51. Academic Press, Limited.

Leveau M., Lochet F., Goutx M., and Blanc F. (1990) Effects of a plume front on the distribution of inorganic and organic matter off the Rhone River. Hydrobiologia 207, 87-93.

Lim E. L., Amaral L. A., Caron D. A., and DeLong E. F. (1993) Application of rRNAbased probes for observing marine nanoplanktonic protists. Applied and Environmental Microbiology 59, 1647-1655.

Lim E. L., Caron D. A., and DeLong E. F. (1996) Development and field application of a quantitative method for examining natural assemblages of protists with oligonucleotide probes. Applied and Environmental Microbiology 62, 1416-1423.

Lipiatou E., Marty J.-C., and Saliot A. (1993) Sediment trap fluxes of polycyclic aromatic hydrocarbons in the Mediterranean Sea. Marine Chemistry 44, 43-54.

Lombardi A. T. and Wangersky P. J. (1991) Influence of phosphorus and silicon on lipid class production by the marine diatom Chaetoceros gracilis grown in turbidostate cage cultures. Marine Ecology Progress Series 77, 39-47.

Mackay D., Mascarenhas R., and Shu W. Y. (1980) Aqueous solubility of polychlorinated biphenyls. Chemosphere 9, 257-264.

Madoni P., Davoli D., and Gorbi G. (1994) Acute toxicity of lead, chromium, and other heavy metals to ciliates from activated sludge plants. Bulletin of Environmental Contamination and Toxicology 53, 420-425.

Madsen E. L., Sinclair J. L., and Ghiorse W. C. (1991) In situ biodegradation: Microbiological patterns in a contaminated aquifer. Science 252, 830-833.

Madsen E. L., Thomas C. T., Wilson M. S., Sandoli R. L., and Bilotta S. E. (1996) In situ dynamics of aromatic hydrocarbons and bacteria capable of $\mathrm{AH}$ metabolism in a coal tar waste-contaminated field site. Environmental Science and Technology 30, 2412-2416.

Mayer A. S., Zhong L., and Pope G. A. (1999) Measurement of mass-transfer rates for surfactant-enhanced solubilization of nonaqueous phase liquids. Environmental Science and Technology 33, 2965-2972.

Mayer L. M., Chen Z., Findlay R. H., Fang J., Sampson S., Self R. F. L., Jumars P. A., Quetel C., and Donard O. F. X. (1996) Bioavailability of sedimentary contaminants subject to deposit-feeder digestion. Environmental Science and Technology 30, 2641-2645.

Mayer P., Halling-Sorensen B., Sijm D. T. H. M., and Nyholm N. (1998) Toxic cell concentrations of three polychlorinated biphenyl congeners in the green alga Selenastrum capricornutum. Environmental Toxicology and Chemistry 17, 18481851. 
McCarthy M., Pratum T., Hedges J., and Benner R. (1997) Chemical composition of dissolved organic nitrogen in the ocean. Nature 390, 150-154.

McGroddy S. E. and Farrington J. W. (1995) Sediment porewater partitioning of polycyclic aromatic hydrocarbons in three cores from Boston Harbor, Massachusetts. Environmental Science and Technology 29, 1542-1550.

Means J. C. (1995) Influence of salinity upon sediment-water partitioning of aromatic hydrocarbons. Marine Chemistry 51, 3-16.

Means J. C. and McElroy A. E. (1997) Bioaccumulation of tetrachlorobiphenyl and hexachlorobiphenyl congeners by Yoldia limatula and Nephtys incisa from bedded sediments: Effects of sediment- and animal-related parameters. Environmental Toxicology and Chemistry 16, 1277-1286.

Mihelcic J. R., Lucking D. R., Mitzell R. J., and Stapleton J. M. (1993) Bioavailability of sorbed- and separate-phase chemicals. Biodegradation 4, 141-153.

Mopper K., Zhou J., Ramana K. S., Passow U., Dam H. G., and Drapeau D. T. (1995) The role of surface-active carbohydrates in the flocculation of a diatom bloom in a mesocosm. Deep-Sea Research II 42, 47-73.

Mopper K., Zhou X., Kieber R. J., Kieber D. J., Sikorski R. J., and Jones R. D. (1991) Photochemical degradation of dissolved organic carbon and its impact on the oceanic carbon cycle. Nature 353, 60-62.

Morrison H. A., Gobas F. A. P. C., Lazar R., Whittle D. M., and Haffner G. D. (1997) Development and verification of a benthic/pelagic food web bioaccumulation model for PCB congeners in western Lake Erie. Environmental Science and Technology 31, 3267-3273.

Munns W. R., Black D. E., Gleason T. R., Salomon K., Bengtson D., and Gutjahr-Gobell R. (1997) Evaluation of the effects of dioxin and PCBs on Fundulus heteroclitus populations using a modeling approach. Environmental Toxicology and Chemistry 16, 1074-1081.

Nagata T. and Kirchman D. L. (1990) Filtration-induced release of dissolved free amino acids: Application to cultures of marine protozoa. Marine Ecology Progress Series 68, 1-5.

Nagata T. and Kirchman D. L. (1992a) Release of dissolved organic matter by heterotrophic protozoa: Implications for microbial food webs. Arch. Hydrobiol. Beih. Ergebn. Limnol. 35, 99-109.

Nagata T. and Kirchman D. L. (1992b) Release of macromolecular organic complexes by heterotrophic marine flagellates. Marine Ecology Progress Series 83, 233-240.

NRC. (1979) Polychlorinated Biphenyls. National Academy of Sciences. 
Parrish C. C., Wangersky P. J., Delmas R. P., and Ackman R. G. (1988) Iatroscanmeasured profiles of dissolved and particulate marine lipid classes over the Scotian slope and Bedford basin. Marine Chemistry 23, 1-15.

Passow U., Alldredge A. L., and Logan B. E. (1994) The role of particulate carbohydrate exudates in the flocculation of diatom blooms. Deep-Sea Research 41, 335-337.

Pelegri S. P., Christaki U., Dolan J., and Rassoulzadegan F. (1999) Particulate and dissolved organic carbon production by the heterotrophic nanoflagellate Pteridomonas danica Patterson and Fenchel. Microbial Ecology 37, 276-284.

Peltzer E. T. and Brewer P. G. (1993) Some practical aspects of measuring DOC sampling artifacts and analytical problems with marine samples. Marine Chemistry 41, 243-252.

Pignatello J. J. (1990) Slowly reversible sorption of aliphatic hydrocarbons in soils. I. Formation of residual fractions. Environmental Toxicology and Chemistry 9 , 1107-1115.

Pomeroy L. R. (1974) The ocean's food web, a changing paradigm. BioScience 24, $499-$ 504.

Pratt J. R. and Cairns J. (1985) Functional groups in the protozoa: Roles in differing ecosystems. Journal of Protozoology 32, 415-423.

Redfield A. C. (1958) The biological controls of chemical factors in the environment. American Scientist 48, 205-221.

Roose P. and Smedes F. (1996) Evaluation of the results of the QUASIMEME lipid intercomparison: The Bligh and Dyer total lipid extraction method. Marine Pollution Bulletin 32, 674-680.

Rubinstein N., Gilliam W. T., and Gregory N. R. (1984) Dietary accumulations of PCBs from a contaminated sediment source by a demersal fish (Leiostomus xanthurus). Aquatic Toxicology 5, 331-342.

Sabaliunas D., Lazutka J., Sabaliuniene I., and Sodergren A. (1998) Use of semipermeable membrane devices for studying effects of organic pollutants: Comparison of pesticide uptake by semipermeable membrane devices and mussels. Environmental Toxicology and Chemistry 17, 1815-1824.

Sackett W. M. (1978) Suspended matter in seawater. In Chemical Oceanography, Vol. 7 (ed. J. P. Riley and R. Chester), pp. 127-172. Academic Press.

Sakugawa H. and Handa N. (1985) Isolation and chemical characteristization of dissolved and particulate polysaccharides in Mikawa Bay. Geochimica et Cosmochimica Acta 49, 1185-1193.

Sanders G., Hamilton-Taylor J., and Jones K. C. (1996) PCB and PAH dynamics in a small rural lake. Environmental Science and Technology 30, 2958-2966. 
Scavia D. (1988) On the role of bacteria in secondary production. Limnology and Oceanography 33, 1220-1224.

Schlautman M. A. and Morgan J. J. (1993) Effects of aqueous chemistry on the binding of polycyclic aromatic hydrocarbons by dissolved humic materials. Environmental Science and Technology 27, 961-969.

Schulz-Bull D. E., Petrick G., and Duinker J. C. (1991) Polychlorinated biphenyls in North Sea water. Marine Chemistry 36, 365-384.

Schwarzenbach R. P., Gschwend P. M., and Imboden D. M. (1993) Environmental Organic Chemistry. John Wiley \& Sons, Inc.

Schwarzenbach R. P. and Westall J. (1981) Transport of nonpolar organic compounds from surface water to groundwater. Laboratory sorption studies. ES\&T 15(1), $1360-1367$.

Schweitzer L. E., Hose J. E., Suffet I. H., and Bay S. M. (1997) Differential toxicity of three polychlorinated biphenyl congeners in developing sea urchin embryos. Environmental Toxicology and Chemistry 16, 1510-1514.

Shen Y.-F., Buikema A. L., Yongue W. H., Pratt J. R., and Cairns J. (1986) Use of protozoan communities to predict environmental effects of pollutants. Journal of Protozoology 33, 146-151.

Sherr B. F., Sherr E. B., and Berman T. (1982) Decomposition of organic detritus: A selective role for microflagellate Protozoa. Limnol. Oceanogr. 27, 765-769.

Sherr B. F., Sherr E. B., and Fallon R. D. (1987) Use of monodispersed, fluorescently labeled bacteria to estimate in situ protozoan bacterivory. Applied and Environmental Microbiology 53, 958-965.

Sherr B. F., Sherr E. B., and Pedros-Alio C. (1989) Simultaneous measurement of bacterioplankton production and protozoan bacterivory in estuarine water. Marine Ecology Progress Series 54, 209-219.

Sherr E. and Sherr B. (1988) Role of microbes in pelagic food webs: A revised concept. Limnol. Oceanogr. 33, 1225-1227.

Sherr E. B. and Sherr B. F. (1994) Bacterivory and herbivory: Key roles of phagotrophic protists in pelagic food webs. Microb. Ecol. 28, 223-235.

Shine J. P. and Wallace G. T. (1995) The formation of surface-active organic complexes of copper in coastal marine water. Marine Chemistry 51, 145-157.

Sikkema J., Bont J. A. M. d., and Poolman B. (1994) Interactions of cyclic hydrocarbons with biological membranes. Journal of Biological Chemistry 269, 8022-8028.

Sinclair J. L., Kampbell D. H., Cook M. L., and Wilson J. T. (1993) Protozoa in subsurface sediments from sites contaminated with aviation gasoline or jet fuel. Applied and Environmental Microbiology 59, 467-472. 
Skoglund R. S., Stange K., and Swackhamer D. L. (1996) A kinetics model for predicting the accumulation of PCBs in phytoplankton. Environmental Science and Technology 30, 2113-2120.

Skoglund R. S. and Swackhamer D. L. (1994) Fate of hydrophobic organic contaminants: Processes affecting uptake by phytoplankton. In Environmental Chemistry of Lakes and Reservoirs (ed. L. A. Baker), pp. 559-574. American Chemical Society.

Stange K. and Swackhamer D. L. (1994) Factors affecting phytoplankton species-specific differences in accumulation of 40 polychlorinated biphenyls (PCBs). Environmental Toxicology and Chemistry 13, 1849-1860.

Stein W. D. (1986) Transport and Diffusion Across Cell Membranes. Academic Press, Inc.

Suffet I. H., Jafvert C. T., Kukkonen J., Servos M. R., Spacie A., Williams L. L., and Noblet J. A. (1994) Influences of particulate and dissolved material on the bioavailability of organic compounds. In Bioavailability: Physical, Chemical, and Biological Interactions (ed. J. L. Hamelink, P. F. Landrum, H. L. Bergman, and W. H. Benson), pp. 93-108. CRC Press.

Swackhamer D. L. and Skoglund R. S. (1993) Bioaccumulation of PCBs by algae: Kinetics versus equilibrium. Environmental Toxicology and Chemistry 12, 831838.

Taylor G., Iturriaga R., and Sullivan C. W. (1985) Interactions of bactivorous grazers and heterotrophic bacteria with dissolved organic matter. Marine Ecology Progress Series 23, 129-141.

Tiehm A., Stieber M., Werner P., and Frimmel F. H. (1997) Surfactant-enhanced mobilization and biodegradation of polycyclic aromatic hydrocarbons in manufactured gas plant soil. Environmental Science and Technology 31, 25702576.

Tranvik L. (1994) Colloidal and dissolved organic matter excreted by a mixotrophic flagellate during bacterivory and autotrophy. Applied and Environmental Microbiology 60, 1884-1888.

Tranvik L. J., Sherr E. B., and Sherr B. F. (1993) Uptake and utilization of 'colloidal DOM' by heterotrophic flagellates in seawater. Marine Ecology Progress Series 92, 301-309.

Tye R., Jepsen R., and Lick W. (1996) Effects of colloids, flocculation, particle size, and organic matter on the adsorption of hexachlorobenzene to sediments. Environmental Toxicology and Chemistry 15, 643-651.

Vojvodic V. and Cosovic B. (1996) Fractionation of surface active substances on the XAD-8 resin: Adriatic Sea samples and phytoplankton culture media. Marine Chemistry 54, 119-133. 